\title{
Unveiling the Cygnus OB2 stellar population with Chandra ${ }^{\star}$
}

\author{
J. F. Albacete Colombo ${ }^{1}$, E. Flaccomio ${ }^{1}$, G. Micela ${ }^{1}$, S. Sciortino ${ }^{1}$, and F. Damiani ${ }^{1}$
}

INAF - Osservatorio Astronomico di Palermo, Piazza del Parlamento 1, 90134 Palermo, Italy
e-mail: facundo@astropa.unipa.it

Received 25 July 2006 / Accepted 10 October 2006

\section{ABSTRACT}

\begin{abstract}
Aims. The aim of this work is to identify the so far unknown low mass stellar population of the $\sim 2$ Myr old Cygnus OB2 star forming region, and to investigate the X-ray and near-IR stellar properties of its members.

Methods. We analyzed a 97.7 ks Chandra ACIS-I observation pointed at the core of the Cygnus OB2 region. Sources were detected using the PWDETECT code and were positionally correlated with optical and near-IR catalogs from the literature. Source events were extracted with the Acis EXTRACT package. X-ray variability was characterized through the Kolmogorov-Smirnov test and spectra were fitted using absorbed thermal plasma models.

Results. We detected 1003 X-ray sources. Of these, 775 have near-IR counterparts and are expected to be almost all associated with Cygnus OB2 members. From near-IR color-color and color-magnitude diagrams we estimate a typical absorption toward Cygnus OB2 of $A_{\mathrm{v}} \approx 7.0 \mathrm{mag}$. Although the region is young, very few stars $(\sim 4.4 \%)$ show disk-induced excesses in the near-IR. $\mathrm{X}$-ray variability is detected in $\sim 13 \%$ of the sources, but this fraction increases, up to $50 \%$, with increasing source statistics. Flares account for at least $60 \%$ of the variability. Despite being generally bright, all but 2 of the 26 detected O- that early B-type stars are not significantly variable. Typical X-ray spectral parameters are $\log N_{\mathrm{H}} \sim 22.25\left(\mathrm{~cm}^{-2}\right)$ and $k T \sim 1.35 \mathrm{keV}$ with $1 \sigma$ dispersion of $0.2 \mathrm{dex}$ and $0.4 \mathrm{keV}$, respectively. Variable and flaring sources have harder spectra with median $k T=3.3$ and $3.8 \mathrm{keV}$, respectively. OB stars are typically softer $(k T \sim 0.75 \mathrm{keV})$. X-ray luminosities range between $10^{30}$ and $10^{31} \mathrm{erg} \mathrm{s}^{-1}$ for intermediate- and low-mass stars, and $2.5 \times 10^{30}$ and between $6.3 \times 10^{33} \mathrm{erg} \mathrm{s}^{-1}$ for OB stars.

Conclusions. The Cygnus OB2 region has a very rich population of low-mass X-ray emitting stars. Circumstellar disks seem to be very scarce. X-ray variability is related to the magnetic activity of low-mass stars $\left(M / M_{\odot} \sim 0.5\right.$ to 3.0$)$ display X-ray activity levels comparable to those of Orion Nebular Cluster (ONC) sources in the same mass range.
\end{abstract}

Key words. stars: formation - stars: early-type - stars: pre-main-sequence - X-rays: stars -

Galaxy: open clusters and associations: individual: Cygnus OB2

\section{Introduction}

The Cygnus OB2 association is one of the richest star forming regions in the Galaxy, containing a large population of $\mathrm{O}$ and B-type stars, some of which are among the most massive stars known (Torres-Dodgen et al. 1991). For its richness Cygnus OB2 has been in the past considered a "young globular cluster" (Knödlseder 2000), although uncertainties remain on the real size of total cluster population (Hanson 2003). At a distance of $1.45 \mathrm{kpc}$ (DM = 10.80, Hanson 2003), Cygnus OB2 lies behind the Great Cygnus Rift and is affected by large and nonuniform extinction, $A_{\mathrm{v}}: 5$ to 15 . Its distance and absorption have so far hindered optical studies of intermediate- and low- mass stars in the region. The position of known massive OB stars in the HR diagram of Cygnus OB2 suggests an age between 1 and $3 \mathrm{Myr}$, with $2 \mathrm{Myr}$ as the most probable value (Massey \& Thompson 1991; Knödlseder et al. 2002) .

The Cygnus OB2 region played a remarkable role in the history of stellar X-ray astronomy as an early X-ray observation, performed with the Einstein satellite and analyzed by Harnden et al. (1979), first revealed that early-type stars are intense X-ray emitters. This work was also the first to suggest the idea that X-rays in OB stars are produced in shocks within stellar winds, the starting point for the X-ray emission model of

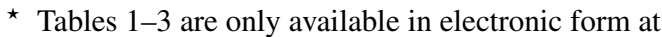
http://www . aanda.org
Lucy \& White (1980). Subsequent ROSAT, ASCA, and ChandraHETG observations of massive stars in the Cygnus OB2 region have improved our understanding of the mechanisms responsible for the X-ray emission of O-type stars (Waldron et al. 2004, 1998; Kitamoto \& Mukai 1996).

These latter studies of the region have focused on the four most known OB-type stars of the region, Cyg\#5, 8, 9, and 12 , but have left the X-ray properties of the other high-, intermediate-, and low-mass stars in the region unexplored. Indeed the intermediate/low-mass population has not even been identified yet. Because of the much higher X-ray luminosity of young stars (e.g. $\log \left(L_{\mathrm{x}}\right) \sim 30-31 \mathrm{erg} / \mathrm{s}$, for $M=1.0-3.0 M_{\odot}$ ) with respect to older field stars (e.g. Getman et al. 2005a), X-ray observations have proved an efficient tool to select likely members of young Star Forming Regions (SFR). Thanks to its high sensitivity and spatial resolution, the Advanced CCD Imaging Spectrometer (ACIS) camera on board the Chandra satellite (Garmire et al. 2003) is particularly suited for this kind of study in crowded stellar fields such as Cygnus OB2.

In this paper we present the analysis of a deep ( $97.7 \mathrm{ks})$ Chandra ACIS observation of Cygnus OB2. In Sect. 2 we introduce our X-ray observation and describe our data calibration and reduction procedure. In Sect. 3 we describe source detection, photon extraction and first characterization of X-ray spectra via hardness ratios. In Sect. 4 we characterize our sources by cross-identifying them with available optical and near-IR 


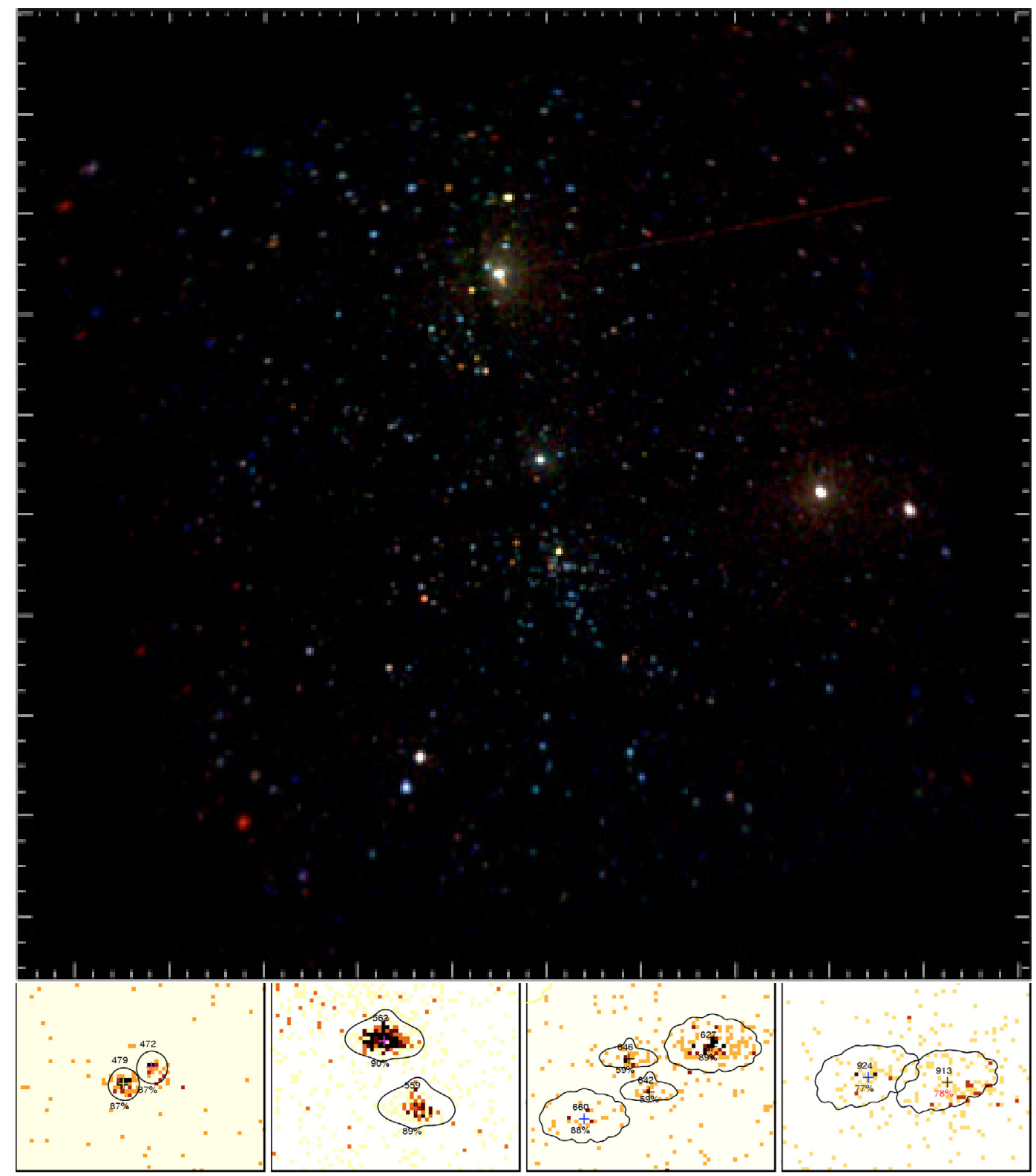

Fig. 1. Upper panel: color-coded ACIS-I image of the $17^{\prime} \times 17^{\prime}$ field in Cygnus OB2 (see color version in the electronic edition). Kernel smoothing was applied to highlight point sources. Energy bands for the RGB image are [0.5:1.7], [1.7:2.8], and [2.8:8.0] keV for the red, green, and blue colors, respectively. The four panels at the bottom show four $30^{\prime \prime} \times 20^{\prime \prime}$ areas of the ACIS field at four different distances from the optical axis: from left to right: $0.7,4.7,5.4$, and 7 arcmin. They illustrate the dependence of the PSF quality on off-axis and show (black continuous lines) the source photon extraction regions established with ACIS EXTRACT. Figures next to these contours indicate the source identification numbers (from Table 1) and the encircled energy fractions.

catalogs. Section 5 deals with X-ray variability while in Sect. 6 the results of the X-ray spectral analysis is presented. In Sect. 7 we discuss out results concerning the X-ray characteristics of high-, intermediate- and low-mass stars of the region. Finally, in Sect. 8 we summarize our results and draw our conclusions.

\section{The X-ray observation}

Cygnus OB2 was observed with the ACIS detector on board the Chandra X-ray Observatory (CXO) (Weisskopf et al. 2002) on 2004 January 16 (Obs.Id. 4511; PI: E. Flaccomio). The total exposure time was $97.7 \mathrm{ks}$. The data were acquired in VERY FAINT mode, to ease filtering of background events, with six CCD turned on, the four comprising the ACIS-I array [0, 1, 2, 3], plus CCDs 6 and 7, part of ACIS-S. However, data from the latter two CCDs will not be used in the following because of the much degraded point spread function (PSF) and reduced effective area resulting from their large distance from the optical axis. The ACIS-I $17^{\prime} \times 17^{\prime}$ field of view (FOV) is covered by 4 chips each with $1024 \times 1024$ pixels (scale $0.49^{\prime \prime} \mathrm{px}^{-1}$ ). The observation was pointed toward $\mathrm{RA}=20^{\mathrm{h}} 33^{\mathrm{m}} 12.2^{\mathrm{s}}$ and $\mathrm{Dec}=+41^{\circ} 15^{\prime} 00.7^{\prime \prime}$, chosen to maximize the number of stars in the FOV and to keep two source-dense regions close to the optical axis, where the PSF is sharper. Figure 1 shows Cygnus OB2 as seen in X-rays by our ACIS-I observation.

\subsection{Data reduction}

Data reduction, starting with the Level 1 event list provided by the pipeline processing at the $\mathrm{CXO}$, was performed using 
CIAO 3.2.2 $2^{1}$ and the CALDB 3.1.0 set of calibration files. We produced a level 2 event file using the ACIS_PROCESS_EVENT CIAO task, taking advantage of the VF-mode enhanced background filtering, and retaining only events with grades = $0,2,3,4,6$ and status $=0$. Photon energies were corrected for the time dependence of the energy gain using the CORR_TGAIN CIAO task. Intervals of background flaring were searched for, but none were found. We will hereafter assume a constant background. To improve the sensitivity to faint sources, given the spectrum of the background and that of typical sources, we filtered out events outside the [500:8000] eV energy band.

An exposure map, needed by the source detection algorithm and to renormalizes source count-rates, was calculated with the CIAO tool MKEXPMAP assuming a monochromatic spectrum $(k T=2.0 \mathrm{keV})^{2}$.

\section{Analysis}

In this section we describe the first steps taken for the analysis of the ACIS-I data. We discuss the detection of X-ray point sources (Sect. 3.1), the definition of source and background event lists (Sect. 3.2), and a first spectral characterization of source spectra through hardness ratios (Sect. 3.3).

\subsection{X-ray source detection}

Source detection was performed with the Palermo Wavelet Detection code, PWDetect ${ }^{3}$ (Damiani et al. 1997b), on the level 2 event list restricted to the [500:8000] eV energy band. PWDetect analyzes the data at different spatial scales, allowing the detection of both point-like and moderately extended sources, and efficiently resolving close sources pairs. The most important input parameter is the detection threshold (SNR), which we establish from the relationship between threshold, background level of the observation, and expected number of spurious detections due to Poisson noise, as determined from extensive simulations of source-free fields (cf. Damiani et al. 1997a). The background level was determined with the BACKGROUND command in the XIMAGE ${ }^{4}$ package. The method amounts to dividing the image into equal-size boxes, discarding those that, according to several statistical criteria, are contaminated by sources, and finally computing the mean level of the remaining ones. We obtain that our ACIS-I observation comprises a total of about $1.58 \times 10^{5}$ background photons. This background level translates into a $S N R$ threshold of 5.2 if we accept one spurious detection in the FOV, or into $S N R>4.5$ if we accept 10 spurious detections. The first choice results in the detection of 868 sources and the second one in 1054 sources. By accepting an extra $\sim 9$ spurious detections in the FOV we thus gain about 177 new reliable sources. Considering moreover that 10 spurious sources amounts to only $\sim 1 \%$ of the total number of detections, we decided to adopt the second less conservative criterion.

After a careful visual inspection of the initial source list, we rejected a total of 51 detections which we considered spurious: 39 were produced by different instrumental artifacts (i.e. out-oftime events, CCD gaps, detector edges, etc.) that were not included in the simulations of source-free fields used to establish

\footnotetext{
${ }^{1}$ http://cxc.harvard.edu/ciao/

2 http://asc.harvard.edu/ciao/download/doc/ expmap_intro.ps

3 See http://www.astropa.unipa.it/progetti_ricerca/ PWDetect

${ }^{4}$ http://heasarc.nasa.gov/xanadu/ximage/ximage.html
}

the SNR threshold, but that are easily recognized. The remaining 12 were multiple detections of the same sources with different spatial scales ${ }^{5}$. In total, our final list of X-ray sources in the Cygnus OB2 region contains 1003 X-ray detections, $99 \%$ of which are expected to be real. The first 7 columns of Table 1 list, for each source: a running source number, name (according to CXC naming convention ${ }^{6}$ ), sky position (RA and Dec) with relative uncertainty, off-axis angle $(\theta)$, significance of the detection (Sig.).

\subsection{Photon extraction}

For further analysis of the detected sources, we used ACIS EXTRACT $^{7}$ (AE) v3.79 (Broos et al. 2002), an IDL based package that makes use of TARA ${ }^{8}$, CIAO and FTOOLS ${ }^{9}$.

The optimal extraction of point source photons is complicated by the non-Gaussian shape of the PSF and by its strong non-uniformity across the ACIS field of view. In particular the width and asymmetry of the PSF depend significantly on the offaxis distance $(\theta)$. The PSF is narrow and nearly circular in the inner $\theta \lesssim 5^{\prime}$ but becomes rapidly broader and more asymmetric at larger off-axis, as demonstrated in the bottom panel of Fig. 1 where we show sources detected in our data at four different offaxis angles.

Extraction from circular regions containing a large fraction (e.g. 99\%) of the PSF would guarantee to collect almost all source photons. However, given the extended wings of the PSF, very large regions would be needed, incurring in the risk of contamination from nearby sources, especially in crowded fields like ours. Moreover the resulting inclusion of a large number of background events would reduce the signal to noise of weak sources. On the other hand, extraction from regions that are too small may reduce the photon statistic for further spectral and timing analysis.

In order to tackle this problem, AE begins with calculating the shape of the model PSF at each source position using the CIAO task MKPSF. It then refines the initial source positions (in our case estimated by PWDetect assuming a symmetric PSF) by correlating the source images with the model PSFs. Following $\mathrm{AE}$ science hints ${ }^{10}$, this last procedure was only used for those sources lying at off-axis larger than 5 arcmin (464 sources), while for the rest of the source (539 sources) we simply adopt data-mean positions. Coordinates listed in Table 1, as well as their $1 \sigma$ uncertainties, are the result of this process. We verified that these positions are an improvement over those computed by PWDetect, especially at large off-axis angles, comparing the offsets between X-ray sources and counterparts in the 2MASS catalog (see Sect. 4.2).

After re-computing positions, AE defines source extraction regions as polygonal contours of the model PSF containing a specified fraction of source events $\left(f_{\mathrm{PSF}}\right)$. Generally, we chose

\footnotetext{
5 This effect occurred for only 3 sources at large off-axis angles, where the PSF is particularly elongated and thus significantly different from the symmetric PSF assumed by PWDetect. Note that, quite obviously, this effect is also not included in the simulations of source-free fields.

6 http://cxc.harvard.edu/cdo/scipubs.html

7 http://www.astro.psu.edu/xray/docs/TARA/ ae_users_guide.html

8 http://www . astro.psu.edu/xray/docs/TARA/

9 http://heasarc.gsfc.nasa.gov/docs/software/ftools/

10 http://www . astro.psu.edu/xray/docs/TARA/ ae_users_guide/node35
} 


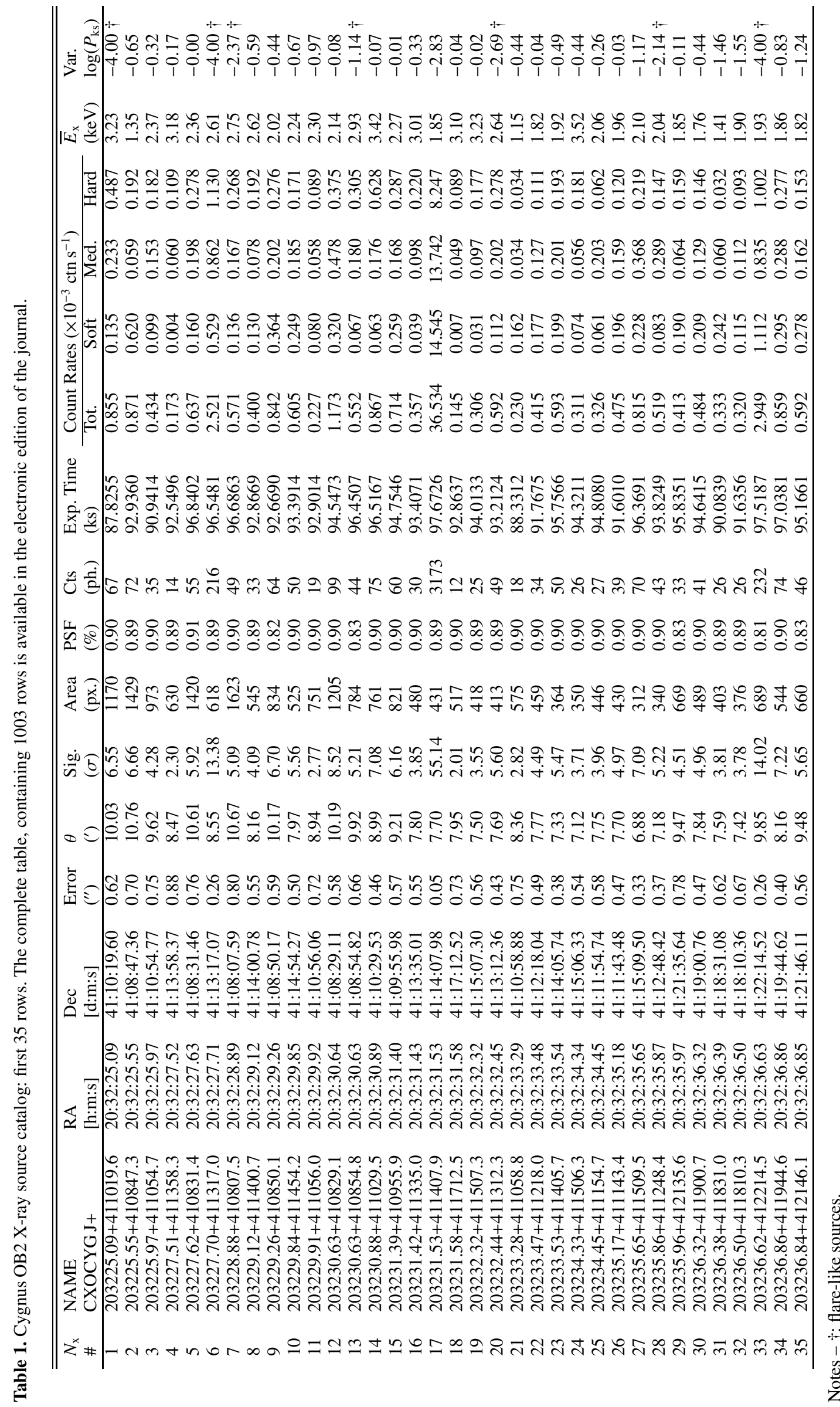



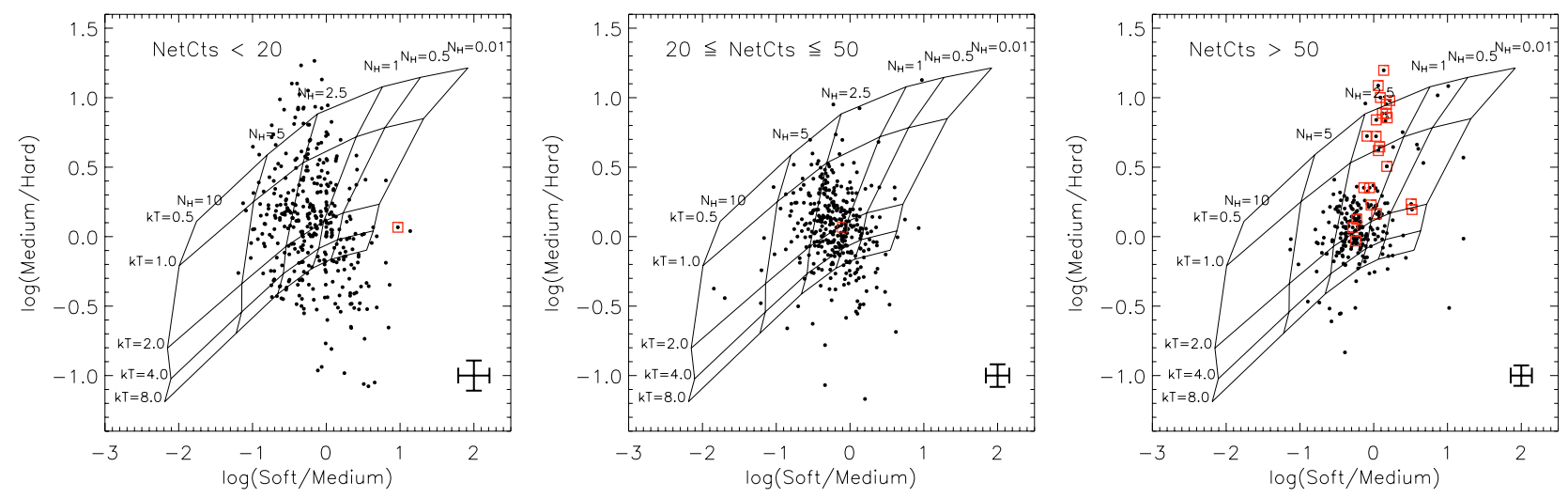

Fig. 2. X-ray color-color diagrams for our ACIS sources. The three panels refer to sources in three ranges of detected counts, as indicated in the upper-left corners. Colors are defined for three energy bands: Soft $(0.5-1.7 \mathrm{keV})$, Medium $(1.7-2.8 \mathrm{keV})$ and Hard (2.8-8.0 keV) keV. The grids refer to predicted colors for Raymond-Smith thermal emission models with different temperatures $(k T=0.5,1.0,2.0,4.0$, and $8.0 \mathrm{keV})$ and affected by varying amounts of absorption, $N_{\mathrm{H}}=0.01,0.5,1.0,2.5,5$, and $10 \times 10^{22} \mathrm{~cm}^{-2}$. Boxes refer to known O-and early B-type stars. Note that the dispersion of points is larger for low-statistics sources. Typical $1 \sigma$ error bars are plotted in the right-bottom part of each panel.

$f_{\mathrm{PSF}}=90 \%$, and computed the contours from the PSF for a mono-energetic source with $E=1.49 \mathrm{keV}$. For $\sim 8 \%$ of the sources in the denser parts of the Cygnus OB2 field this fraction was reduced to avoid contamination with other nearby sources, in the most extreme cases down to $f_{\mathrm{PSF}} \sim 40 \%$. The four panels at the bottom of Fig. 1 show examples of extraction regions. Otherwise, we have detected only six sources: Id.\# 17, 60, 488, $544,568,729$ that suffers of a pile-up fraction $\left(f_{\text {pile-up }}\right)$ of $3.96 \%$, $17.7 \%, 11.6 \%, 2.39 \%, 15.6 \%$ and $4.6 \%$, respectively. In these cases, we extracted events in annular regions that exclude the PSF cores for all sources that exhibits a $f_{\text {pile-up }}$ greater than $2 \%$. The inner radii was fixed at $2 \operatorname{arcsec}^{11}$, while the outer circles were choosen as the smallest that inscribe the $f_{\mathrm{PSF}}=99 \%$ contours.

Although the ACIS-I instrumental background level is spatially quite uniform, the actual observed background varies substantially across the crowded Cygnus OB2 field due to the extended PSF wings of bright sources and to their readout trails. The background was therefore estimated locally for each source, adopting once again the automated procedure implemented in $\mathrm{AE}$, which defines background extraction regions as circular annuli with inner radii 1.1 times the maximum distance between the source and the $99 \%$ PSF contour, and outer radii defined so that the regions contain more than 100 "background" events. In order to exclude contamination of the regions by nearby sources, background events are defined from a "swiss cheese" image that excludes events within the inner annuli radii of all the 1003 sources.

Results of the photon extraction procedure are listed in Cols. $8-16$ of Table 1 . We give: the source extraction area (Col. 8); the PSF fraction within the extraction area, assuming $E=1.49 \mathrm{keV}$ (9); the background-corrected extracted source counts in the $0.5-8.0 \mathrm{keV}$ band (10); the exposure time (11), the count rates $(\mathrm{CR})$ in four spectral bands computed as the ratio between the source photons (corrected for $f_{\mathrm{PSF}}$ ) and the exposure time $(12-15)$; the median photon energy $\left(\overline{E_{\mathrm{x}}}\right),(16)$.

In summary, our $1003 \mathrm{X}$-ray detections span a wide count range, from 4 to $\sim 15000$ photons. Most sources are faint (e.g. $42 \%$ have less than 20 photons). The lower envelope of the counts vs. off-axis plot (not shown) indicates that the minimum

\footnotetext{
11 http://www.astro.msfc.nasa.gov/Ch4/ Ch4_15-03_Tsujimoto.pdf
}

number of photons necessary for detection ranges from 4 on axis to $\sim 20$ close to the detector corners $(\theta=10 \mathrm{arcmin})$.

\subsection{X-ray hardness ratios}

In order to characterize the X-ray spectra of low-statistic sources it is common practice to use the ratios of source counts in different spectral bands, i.e. X-ray hardness ratios (XHR), or the logarithm of these values which can be considered "X-ray colors" (Schulz et al. 1989; Prestwich et al. 2003).

We use this method, dividing the full energy range into three bands: Soft $\left(S_{\mathrm{x}}: 0.5-1.7 \mathrm{keV}\right)$, Medium $\left(M_{\mathrm{x}}: 1.7-2.8 \mathrm{keV}\right)$ and Hard $\left(H_{\mathrm{x}}: 2.8-8.0 \mathrm{keV}\right)$. Figure 2 shows, separately for sources in three detected counts ranges, the "Hard X-ray color" $\left(\log \left[M_{\mathrm{x}} / H_{\mathrm{x}}\right]\right)$ vs. the "Soft X-ray color" $\left(\log \left[S_{\mathrm{x}} / M_{\mathrm{x}}\right]\right)$. For reference we also plot the predicted loci for absorbed thermal sources with plasma temperatures between 0.5 and $8.0 \mathrm{keV}$ and $N_{\mathrm{H}}$ between $10^{20}$ and $10^{23} \mathrm{~cm}^{-2}$. The grid was calculated with the Portable Interactive Multi-Mission Simulator $\left(\mathrm{PIMMS}^{12}\right)$ for a Raymond-Smith (RS) emission model (Raymond \& Smith 1977).

A comparison of the three panels in Fig. 2 shows that the position of sources with respect to the $k T-N_{\mathrm{H}}$ grid is significantly affected, other than by the source spectra, by the source statistic. The positions of individual sources with less than 20 counts (left panel) are, for example, considerably spread out because of large statistical uncertainties. Sources with higher statistics $(20 \leq$ NetCnts $\leq 50$, central panel) are more concentrated around $k T \sim 2.0 \mathrm{keV}$ and $N_{\mathrm{H}} \sim 2.3 \times 10^{22} \mathrm{~cm}^{-2}$. Sources with more than 50 photons (right panel) are even more concentrated around these same values of $k T$ and $N_{\mathrm{H}}$. A separate group of soft-spectrum sources however becomes visible in the upper part of the grid, with typical $k T \sim 0.8 \mathrm{keV}$. Most of these sources are associated with known early type stars, indicated by squares in Fig. 2. Others, with somewhat smaller $N_{\mathrm{H}}$, are likely foreground stars as also argued in Sect. 4.2.

\section{Optical and near-IR counterparts}

Cygnus OB2 has been the target of numerous optical studies for more than 50 years (Johnson \& Morgan 1954; Schulte 1958).

\footnotetext{
12 http://heasarc.gsfc.nasa.gov/docs/software/tools/ pimms.html
} 
Table 2. Near-IR counterparts of Cygnus OB2 X-ray sources and near-IR stellar parameters: first rows. The complete version is available in the electronic edition of the journal.

\begin{tabular}{|c|c|c|c|c|c|c|c|c|}
\hline \multirow{2}{*}{$\begin{array}{l}N_{\mathrm{x}} \\
\#\end{array}$} & \multicolumn{2}{|c|}{ X-ray - 2MASS counterpart } & \multirow{2}{*}{$\begin{array}{l}\text { Off. } \\
\left({ }^{\prime \prime}\right)\end{array}$} & \multicolumn{4}{|c|}{ 2MASS photometry } & \multirow{2}{*}{$\begin{array}{l}A_{\mathrm{v}}^{\ddagger} \\
(\mathrm{mag})\end{array}$} \\
\hline & CXOAC J+ & 2MASS J+ & & $J$ & $H$ & $K_{\mathrm{s}}$ & Ph. qual $^{\dagger}$ & \\
\hline 1 & $203225.09+411019.6$ & - & - & - & - & - & - & - \\
\hline 2 & $203225.55+410847.3$ & $20322545+4108473$ & 1.56 & $14.76 \pm 0.06$ & $14.23 \pm 0.07$ & $13.95 \pm 0.07$ & AAA & 8.54 \\
\hline 3 & $203225.97+411054.7$ & $20322597+4110547$ & 0.24 & $15.24 \pm 0.05$ & $13.65 \pm 0.03$ & $12.69 \pm 0.03$ & AAA & 7.71 \\
\hline 4 & $203227.51+411358.3$ & $20322756+4114001$ & 1.61 & $16.00 \pm 0.06$ & $14.46 \pm 0.04$ & $13.90 \pm 0.06$ & AAA & 0.16 \\
\hline 5 & $203227.62+410831.4$ & $20322754+4108336$ & 2.41 & $15.91 \pm 0.06$ & $14.39 \pm 0.03$ & $13.82 \pm 0.05$ & AAA & N/A \\
\hline 6 & $203227.70+411317.0$ & $20322768+4113169$ & 0.58 & $14.96 \pm 0.03$ & $13.49 \pm 0.02$ & $12.88 \pm 0.03$ & AAA & 2.21 \\
\hline 7 & $203228.88+410807.5$ & - & - & - & - & - & - & - \\
\hline 8 & $203229.12+411400.7$ & $20322913+4114012$ & 0.26 & $16.74 \pm 0.16$ & $15.36 \pm 0.08$ & $14.72 \pm 0.11$ & CAB & 9.01 \\
\hline 9 & $203229.26+410850.1$ & $20322928+4108494$ & 1.03 & $12.26 \pm 0.01$ & $11.49 \pm 0.01$ & $11.07 \pm 0.01$ & AAA & 8.52 \\
\hline 10 & $203229.84+411454.2$ & $20322985+4114536$ & 0.83 & 17.44 & $15.43 \pm 0.10$ & $14.37 \pm 0.09$ & UAA & N/A \\
\hline 11 & $203229.91+411056.0$ & $20322994+4110575$ & 1.29 & $15.26 \pm 0.05$ & $14.04 \pm 0.04$ & $13.53 \pm 0.04$ & AAA & N/A \\
\hline 12 & $203230.63+410829.1$ & $20323051+4108284$ & 2.19 & $15.47 \pm 0.07$ & 13.71 & 13.09 & AUU & 4.19 \\
\hline 13 & $203230.63+410854.8$ & $20323064+4108565$ & 1.49 & $15.31 \pm 0.05$ & $13.68 \pm 0.03$ & $13.01 \pm 0.03$ & AAA & 5.43 \\
\hline 14 & $203230.88+411029.5$ & - & - & - & - & - & - & - \\
\hline 15 & $203231.39+410955.9$ & $20323143+4109558$ & 0.58 & $14.52 \pm 0.05$ & 13.04 & 12.46 & AUU & 9.69 \\
\hline
\end{tabular}

$\dagger$ MASS photometric quality flags for the $J, H$ and, $K_{\mathrm{s}}$ bands: "A" to " $\mathrm{D}$ " indicate decreasing quality of the measurements, " $\mathrm{U}$ " that the value is an upper limit. See 2 MASS documentation for details. $\$ \mathrm{~N} / \mathrm{A}$ indicates stars with unconstrained $A_{\mathrm{v}}$ (see text).

Massey \& Thompson (1991) identified 120 candidate massive members in an area of about $0.35 \mathrm{deg}^{2}$, on the basis of UBV photometry, and gave optical spectral classifications for over 70 OB stars. More recently, Hanson (2003) published new spectral classification for 14 more $\mathrm{OB}$ candidates, improving the massive star census of Cygnus OB2, and the estimates for its distance $(1450 \mathrm{pc})$ and age ( 2 Myr). A total of 33 OB stars from the catalog of Hanson (2003) lie in the $0.0823 \mathrm{deg}^{2}$ FOV of our $\mathrm{X}$-ray observation and were thus cross identified with our X-ray source list (Sect. 3.2). Due to the high and variable absorption in the Cygnus OB2 line of sight, and to the relative shallowness of the available optical catalogs, the correlation between X-ray sources and optical catalogs is of limited use for the study of intermediate- and low-mass stars. We thus decided to base our characterization of the X-ray sources mostly on near-IR data, for which the impact of dust extinction is reduced and is comparable to that in the X-ray band. We made use of $J(1.25 \mu \mathrm{m})$, $H(1.65 \mu \mathrm{m})$ and $K_{\mathrm{s}}(2.17 \mu \mathrm{m})$ photometry from the Two Micron All Sky Survey (2MASS) Point Source Catalog (PSC) ${ }^{13}$. 2MASS is complete to magnitudes of $15.8,15.1$ and $14.3 \mathrm{mag}$ in the $J, H$ and $K_{\mathrm{s}}$ bands, respectively. We limited our analysis to $2 \mathrm{MASS}$ sources for which the quality flag for at least one of the three magnitudes is equal to A, B, C or D (cf. the 2MASS All-Sky Data Release User's Guide). With this restriction 11 sources were removed from our initial list of 50612 MASS point sources in the ACIS FOV, leaving a total of 5050 objects.

\subsection{Cross-identification}

We began by cross identifying our X-ray source list with the $2 \mathrm{MASS}$ catalog. Identification radii, $R_{\text {id }}$, were chosen to limit the number of spurious identifications due to chance alignments, $N_{\text {chance, }}$ and at the same time to include a large number of the true physical associations, $N_{\text {true }}$. First we estimated, as a function of $R_{\text {id }}$, the number of chance identifications expected in a given sky area, $A_{\text {search }}$, assuming (i) uncorrelated NIR and X-ray positions and (ii) a uniform surface source density of the $N_{2 \text { MASS }}$ 2MASS sources lying in the search

\footnotetext{
13 See http://www.ipac.caltech.edu/2mass
}

area: $N_{\text {chance }}\left(R_{\mathrm{id}}\right)=N_{\mathrm{X}} A_{\text {id }} N_{2 \mathrm{MASS}} / A_{\text {search }}$, where $A_{\text {id }}=\pi R_{\text {id }}^{2}$ is the area of identification circles. $N_{\text {true }}\left(R_{\text {id }}\right)$ can instead be estimated from the observed total number of identifications at any given $R_{\text {id }}$ as $N_{\text {id }}\left(R_{\text {id }}\right)-N_{\text {chance }}\left(R_{\text {id }}\right)$. We chose the identification radius as the largest for which $N_{\text {true }}>N_{\text {chance. }}$.

Because the Chandra PSF, and therefore the position uncertainty of X-ray sources, depends mainly on the off-angles, we perform this analysis in four different ranges of off-angles: $[0-2),[2-4),[4-7)$ and $>7$ arcmin. Our identification radii in these four regions are 1.0, 1.5, 2.1, and 2.7 arcsec, respectively.

Before performing the final identifications we searched for possible systematic differences between the X-ray and 2MASS positions. We first performed a preliminary cross-identification and compared the coordinates of identified pairs. A small systematic offset between the two catalogs was found. We thus shifted the Chandra coordinates and performed a new crossidentification, repeating this process iteratively until the offset was reduced to $\sim 0.01$ arcsec, i.e. much smaller than the statistical errors on source positions. In the end the offset between the two catalogs was: $\Delta\left(\mathrm{RA}_{\mathrm{x}-2 \mathrm{MASS}}\right)=+0.12^{\prime \prime} \pm 0.10^{\prime \prime}$ and $\Delta\left(\right.$ Dec $\left._{\mathrm{x}-2 \mathrm{MASS}}\right)=+0.49^{\prime \prime} \pm 0.17^{\prime \prime}$. The result of the final identification is shown in Table 2, where we list, in the first 7 columns, the X-ray and near-IR identifiers of cross-identified sources, the offset between the two positions, and the $J, H, K_{\mathrm{s}}$ magnitudes from 2MASS. A total of $775 \mathrm{X}$-ray sources out of the 1003 in our list were identified with 2 MASS objects. Three X-ray sources (\#12, \#148, and \#449) were identified with two 2MASS objects but, after checking the positions visually, we adopted the closer of the counterparts. The fraction of identified X-ray sources appears to increase as we move to larger off-angles. In the four annular regions defined above, these fractions are: $76 / 116(65 \%)$, $227 / 304$ (74\%), 294/374 (79\%) and 178/209 (85\%), in order of increasing off-angles. This trend can be attributed to the already mentioned (3.2) dependence of the ACIS-I sensitivity on the offaxis angle and to the fact that more intense X-ray sources are more likely to have a near-IR counterpart than fainter ones.

With respect to the expected number of chance identifications we estimate, for off-axis ranges [0:2), [2:4), [4:7), and $>7$ arcmin, and with the formula given above, no more than 1.6, $7.9,16.4$, and 12.5 , respectively for a total of $\leq 39$. Note however 
that the assumption that X-ray and 2MASS source lists are fully uncorrelated is not true (other than for the 10 expected spurious detections), so that this number should be considered as a loose upper limit (cf. Damiani et al. 2003).

We estimate the expected number of extragalactic sources in our detection list following Flaccomio et al. (2006). We consider the ACIS count-rates of non-stellar sources in the Chandra Deep Field North (CDFN, Alexander et al. 2003; Barger et al. 2003) and estimate absorption corrected count-rates assuming $N_{\mathrm{H}}=1.54 \times 10^{22}$ (from $A_{\mathrm{v}}=7.0$, see Sect. 4.2) using PIMMS and assuming power-law spectra with index $\Gamma$. We then compare these count rates with upper limits taken at random positions in the ACIS FOV. For $\Gamma$ between 1 and 2 we obtain 61 to 87 expected extragalactic sources. Given the intrinsic near-IR fluxes of these sources and the absorption toward Cygnus OB2, they are expected to be among the 228 without NIR counterparts (cf. Flaccomio et al. 2006).

We identified our X-ray source list with the 33 early type stars listed by Hanson (2003) and lying in our ACIS FOV. Of these, all the 20 O-type stars associated with an X-ray source, while of the 13 B-type stars only 6 are detected.

\subsection{Near-IR properties of the $X$-ray sources}

We now investigate the NIR properties of the X-ray sources with 2MASS counterparts. For this purpose we restrict our analysis to sources with high quality photometry, i.e. those for which the quality flag (see 2MASS documentation) is " $A A A$ ", or for which uncertainties on the $J, H$ and $K_{\mathrm{s}}$ magnitudes are all lower than $0.1 \mathrm{mag}$. With these requirements the total number of IR sources in the ACIS FOV is reduced from 5050 to 2187 . Counterparts of $\mathrm{X}$-ray sources were selected only on the basis of their magnitude errors ( $<0.1 \mathrm{mag}$ ), yielding 519 sources out of the original 775 .

Figure 3 shows the $J-H$ vs. $H-K_{\mathrm{s}}$ color-color $(\mathrm{CC})$ diagram for all the selected 2MASS objects in the ACIS FOV, both X-ray detected and undetected. We also plot for comparison the MS (Kenyon \& Hartmann 1995), the Classical T-Tauri Stars (CTTS) locus of Meyer et al. (1997), and three reddening vectors starting from these loci and with slope $\left(A_{\mathrm{Ks}} / E(H-\right.$ $\left.\left.K_{\mathrm{s}}\right)=0.125\right)$ taken from the extinction law given by Hanson (2003). Cygnus OB2 members with purely photospheric emission should lie in this reddening band. However, as the line of sight toward Cygnus OB2 is not far from that to the Galactic center, many field interlopers are also expected in the same band. Young stellar objects (YSOs), such as Classical T Tauri and Herbig $\mathrm{Ae} / \mathrm{Be}$ stars, because of the NIR excess emission originating in the inner parts of their circumstellar disks, are often found to the right of this band, i.e. in the CTTS locus.

However, very few X-ray sources, i.e. likely Cygnus OB2 members, have colors consistent with the (reddened) CTTS locus. If we neglect the uncertainties on data points, 23 sources lie in the reddening band of the CTTS locus. We do not include here the peculiar supergiant B5 Ie Cyg\#12 and discuss its nature in Sect. 7.1. A total of 23 stars in the CTTS reddening band means a fraction of $23 / 519 \sim 4.4 \%$ of all the X-ray sources in the CC diagram. We compare this fraction with the one observed in the ONC, adopting Chandra Orion Ultradeep Project (COUP) sources in the same mass range ${ }^{14}$ that we reach in Cygnus OB2 (i.e. $M \geq 1 M_{\odot}$, cf. Sect. 7): $19 / 92 \sim 20.6 \%$. The difference between the two fractions of

14 We only consider COUP sources for which mass estimates are given by Getman et al. (2005a).

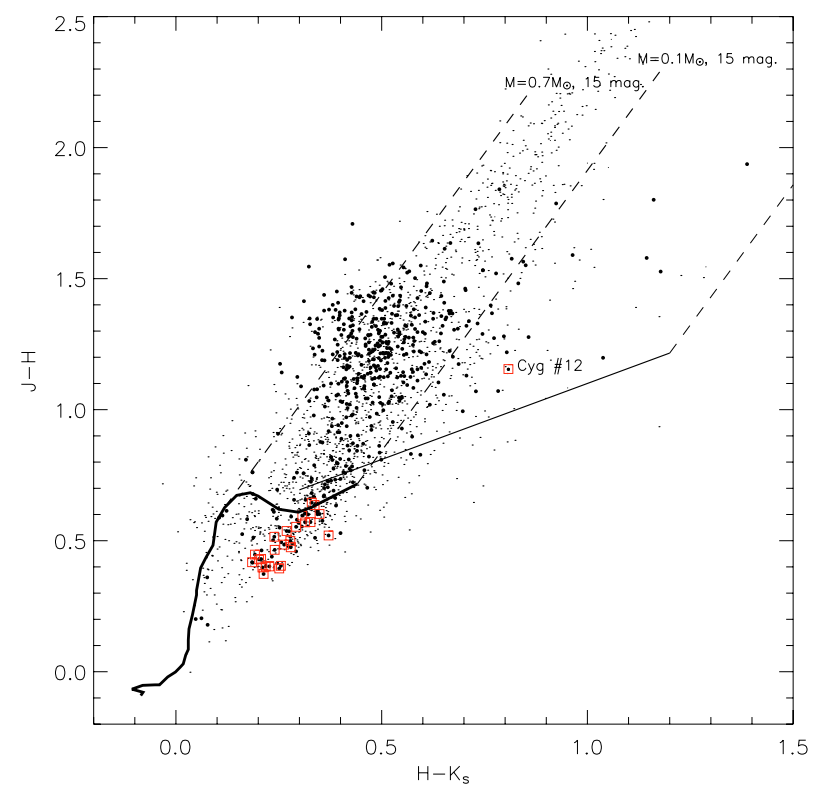

Fig. 3. $J H K_{\mathrm{s}}$ color-color diagram of objects in the $17^{\prime} \times 17^{\prime}$ Cygnus OB2 FOV with high quality 2MASS photometry. Filled circles and small points refer to X-ray detected and undetected 2MASS sources, respectively. Detected known O- and B-type stars are indicated by squares. We also show for reference the main-sequence from Kenyon \& Hartmann (1995), the CTTS locus of Meyer et al. (1997) and reddening vectors (dashed lines) with length corresponding to $A_{\mathrm{v}}=15 \mathrm{mag}$. Note: i) the low number of X-ray sources lying in the CTTS loci; ii) the peculiar position of Cyg \#12 (labeled), one of most intrinsically luminous stars in the Galaxy, indicative of a high reddening.

stars with near-IR detected disks is significant, a factor of $\sim 4.7$. It could be for two different reasons:

- Cygnus OB2 is older than the ONC ( 2 vs. $\sim 1$ Myr) and its disks might have dissipated. According to Hillenbrand (2005), the fraction of stars with disks detectable in the nearIR should decrease between 1 and 2 Myr by a factor of 2-3. This, together with the statistical uncertainties in the disk fractions might explain the observed difference ${ }^{15}$.

- The effect of disk photo-evaporation by the intense UV radiation field of the hot massive OB stars. This mechanism, predicted theoretically (e.g. Adams et al. 2004) and observed, e.g., in the ONC proplyds (e.g. Bally et al. 2000), is thought to be effective in massive star forming regions, such as Cygnus OB2, having a large number of UV emitting Oand B-type stars. A shortening of the disk lifetime with decreasing distance from $\mathrm{O}$ - stars has been recently observed in the massive star forming region NGC 6611 (Guarcello et al. 2006, in prep.).

Figure 4 shows the $K_{\mathrm{s}}$ vs. $H-K_{\mathrm{s}}$ color magnitude (CM) diagram for the same stars plotted in Fig. 3. We also show for reference the expected cluster locus: the intrinsic $K_{\mathrm{s}}$ magnitudes and $H-K_{\mathrm{s}}$ colors for stars earlier than B5V were taken from the MS calibration of Knödlseder (2000) and Bessell \& Brett (1989). For later spectral types (masses between 0.1 and $7 M_{\odot}$ ), we adopted

\footnotetext{
15 A direct comparison with the results of Hillenbrand (2005) is however not possible because her estimate of disk lifetime (i) refers to star in the $0.3-1.0 M_{\odot}$, i.e. less massive than the ones we observe in Cygnus OB2, (ii) is based on a different, and more efficient, indicator of disks presence than the one we can use here, i.e. the $H-K$ color excess measured with respect to the photospheric value as determined from spectral types.
} 


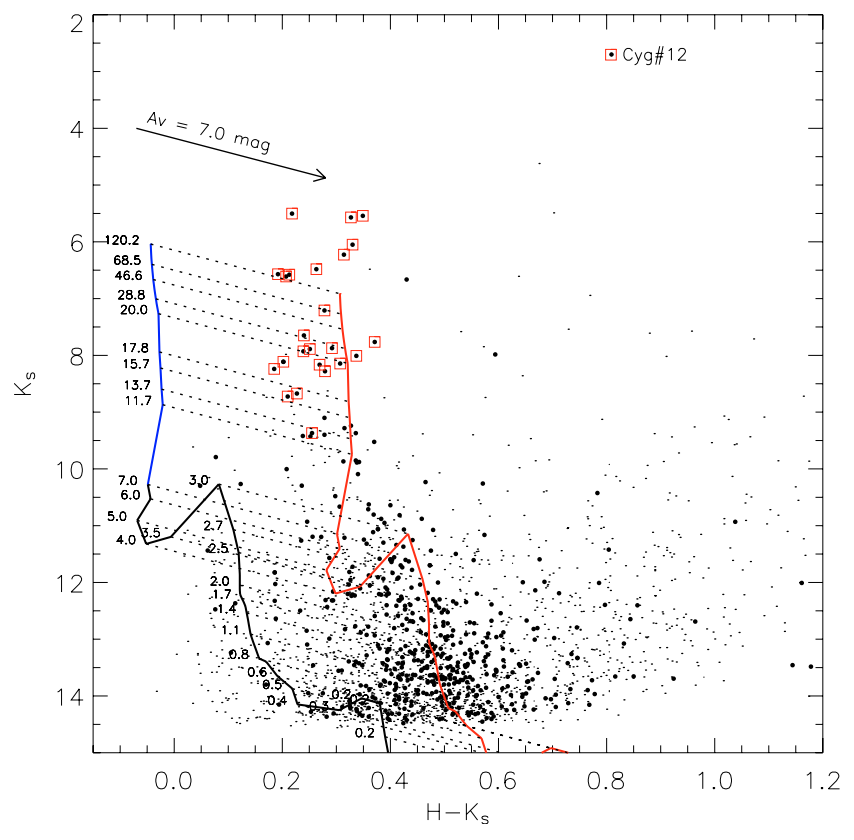

Fig. 4. CM diagrams of the Cygnus OB2 region. Symbols as in Fig. 3. The two parallel curves indicate the expected cluster loci for the assumed distance and for a mean reddening of $A_{\mathrm{V}}=0.0$ and 7.0 mag. They represent the MS for (un-reddened) $K_{\mathrm{s} 0}<10.3$ and the $2 \mathrm{Myr}$ isochrone for fainter magnitudes.

the 2 Myr isochrone from Siess et al. (2000), converted to the observational plane using the calibration given by Kenyon \& Hartmann (1995). The adopted MS and 2 Myr isochrone overlap satisfactorily.

In order to estimate the typical visual absorption of cluster members we calculated the distance of each X-ray source to the cluster locus along the reddening direction. Resulting $A_{\mathrm{v}}$ values for individual sources are listed in the last column of Table 2 . Note that for $10.25<K_{\mathrm{s} 0}<11.35$ the absorption cannot be constrained because the reddening vector intersects the cluster locus more than once. We computed median absorption values in two luminosity ranges: for $K_{\mathrm{s} 0}>11.35$ we obtained $A_{\mathrm{v}}=7.0 \mathrm{mag}$, while for $K_{\mathrm{s} 0}<10.25$, considering only the known OB stars, we obtained $A_{\mathrm{v}}=5.63 \mathrm{mag}$. We note that the above estimations depend on the reliability of the assumed cluster locus and on the assumption that the $H$ and $K$ magnitudes are not significantly affected by disk-induced excesses. As observed above, given the paucity of stars with excesses, the latter appears to be a good approximation for Cygnus OB2 stars. It is comforting that our estimate for the OB type stars is in fairly good agreement with the median of the extinctions computed by Hanson (2003) using photometric and spectroscopic data: $A_{\mathrm{v}}=5.7 \mathrm{mag}$. The lower extinction derived for high mass stars than to lower mass ones may indicate that the strong winds and radiation field of massive stars may have cleared their surrounding environment.

In both the CC and CM diagrams, some X-ray sources, of the order of 15-20, lie close to the un-reddened cluster loci. These are likely to be foreground MS stars and thus to contaminate the sample of X-ray detected cluster members. This conclusion is corroborated by the X-ray hardness-ratio analysis: eight of these stars have soft and relatively unabsorbed spectra (the stars to the right of the $\mathrm{OB}$ stars, in the upper-right corner of Fig. 2-right), as expected from foreground field stars.

In the following (Sect. 7) we will correlate the X-ray properties of our sources with stellar parameters derived from the available optical and near-IR data. We obtain an estimate of stellar masses for 682 2MASS counterparts from the $J$-band magnitudes (limited to quality flags "A" to "D") and the mass vs. $J$ mag. relationship appropriate for the cluster age, distance and extinction, obtained as described above for the cluster locus in the $H, H-K$ diagram $^{16}$. The relation is degenerate in two ranges of $J$, corresponding to $0.2-0.4 M_{\odot}$ and $2.8-5.3 M_{\odot}$. Four and $28 \mathrm{X}$-ray detected stars lie in these ranges, respectively.

\section{Temporal variability}

The temporal variability of young low mass sources is often complex: the most common phenomena are magnetic flares with rapid rise and slower decays, superposed on an apparently constant or, sometimes, rotationally modulated emission (Flaccomio et al. 2005; Wolk et al. 2005). Other forms of variability, with less clear physical origin, are often observed.

We first investigated X-ray variability using the non-binned one-sample Kolmogorov-Smirnov (KS) test (Press et al. 1992). This test compares the distribution of photon arrival times with that expected for a constant source. The test was applied to photons in the source extraction regions, which also contain background photons. Given that the background was found to be low and constant (Sect. 2.1), the results, i.e. the confidence with which we can reject the hypothesis that the flux was constant during our observation, can be attributed to the source photons. Table 1, Col. 17, reports the logarithm of the KS-test significance with values $<-4$ truncated at that value: sources with $\log \left(P_{\mathrm{KS}}\right)<-3.0$ can be considered almost definitively variable as we expect at most one of the 1003 sources (i.e. $0.1 \%$ ) to be erroneously classified as variable. Eighty-five X-ray sources $(\approx 8.5 \%$ of the total) fall in this category. Sources with $-2.0<$ $\log \left(P_{\mathrm{KS}}\right)<-3.0$ can be considered as likely variable, but up to 9 such sources (on average) might actually be constant. Forty-nine Cygnus OB2 sources fall in this category.

These numbers of sources in which variability is detected are a lower limit to the total number of variable sources in the region for several reasons: i) most of the observed variability is in the form of flares, i.e. events that are shorter than our observation and with a duty-cycle that may be considerably longer (Wolk et al. 2005); ii) the sensitivity of statistical tests to time variability of a given relative amplitude depends critically on photon statistics. This is illustrated in Fig. 5, where we plot the fraction of variable sources as a function of source counts: the clear correlation between the two quantities is most likely due to this statistical bias even though we cannot exclude a real dependence.

Next, we extracted binned light-curves for each of our Cygnus OB2 X-ray sources adopting a bin length of $600 \mathrm{~s}$, a compromise between bins that are long enough to reach a good signal-to-noise ratio per bin for most sources and sufficiently short to resolve the decay phase of typical flares. Since the background of our observation is both low (negligible for many sources) and constant, we did not apply any background subtraction. We inspected the 857 light-curves of sources with $>10$ photons, finding flare-like events, qualitatively defined as a rapid rise and a slow decay, in 98 sources, i.e. $\approx 9.8 \%$ of the cases. These sources are indicated by a $\dagger$ in the last column of Table 1 . Of these 98 sources, $66(\sim 65 \%)$ have a $\log \left(P_{\mathrm{KS}}\right) \leq-3.0$, while 13 were classified as probably variable $\left(-2.0<\log P_{\mathrm{KS}} \leq-3.0\right)$.

\footnotetext{
16 The use of the $J$-band is justified because (i) in the presence of disk excesses the $J$-band is the most representative of the photospheric emission and (ii) the mass ranges in which the mass-luminosity relationship is degenerate are narrower than for a similar relationship in the $H$ and $K$ bands.
} 


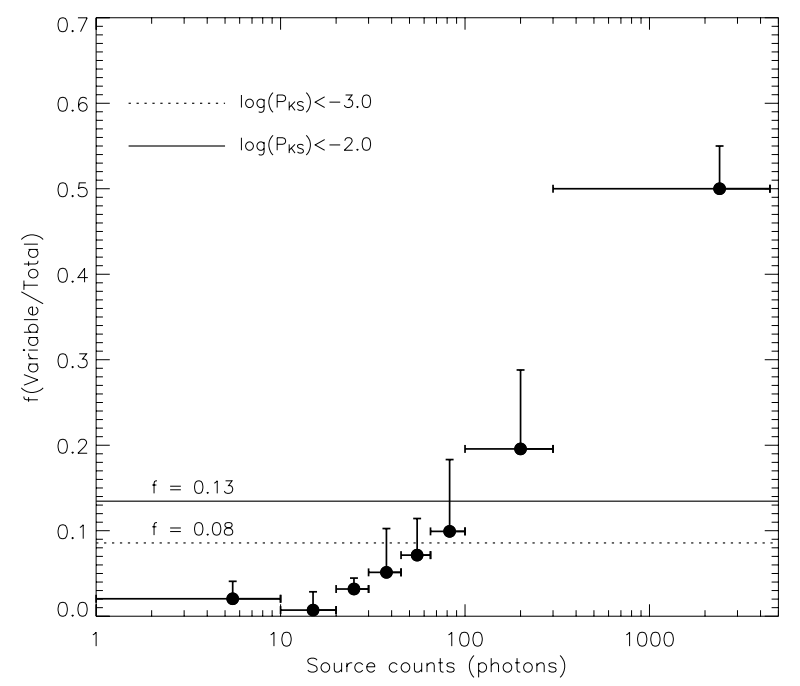

Fig. 5. Fraction of variable sources as a function of source counts. Horizontal bars indicate the range of source counts. Filled circles indicate, for each counts bin, the fraction of sources with $\log \left(P_{\mathrm{KS}}\right)<-3.0$ (85 sources in total), while the upper tip of the vertical bars indicate the fraction with $\log \left(P_{\mathrm{KS}}\right)<-2.0$ (134 sources in total). The continuous and dashed horizontal lines indicate the overall (average) variability fractions obtained for the two confidence levels.

The remaining 19 sources were not detected as variable by the $\mathrm{KS}$ test $\left(\log P_{\mathrm{KS}}>-2.0\right)$ and may or may not be actually "flaring". Figure 6 shows light-curves for 20 variable sources, 14 of which are classified as flaring. Some of these sources (e.g. \#1, \#676 and \#911) experience "impulsive" flares with very quick rises and decay phases of only a few hours. Others (e.g. \#33, $\# 172$, and \#260) show longer (2 to $10 \mathrm{~h}$ ) flares. In several instances a second impulsive event is visible during the exponential decay of a previous flare (e.g. sources \#6, \#52, \#439, \#600, $\# 796$, and \#945). Other sources (e.g. \#555, \#834 and \#863) have variable light-curves that bear little resemblance to typical flares and are instead characterized by slow continuous rises or decays that might be explained by rotational modulation of nonhomogeneously distributed plasma (Flaccomio et al. 2005).

As expected if flares originate from magnetic reconnection events (Favata \& Micela 2003), the median photon energies for flaring sources are generally higher than those of non-flaring sources: the distribution of median energies for the flaring source peaks at $\bar{E}_{x} \approx 2.6 \mathrm{keV}$, with a $1 \sigma$ dispersion of $0.4 \mathrm{keV}$, while for non-variable stars it peaks at $\bar{E}_{x} \approx 2.1 \mathrm{keV}$, with a $1 \sigma$ dispersion of $0.3 \mathrm{keV}$. A similar conclusion can be drawn from the spectral modeling presented in the next section (Sect. 6): flaring sources often require higher temperature models than non-variable ones.

The variability of massive $\mathrm{O}$ and early B-type stars is significantly different from that of low mass members. Among the 26 OB stars detected in our FOV, 24 are classified as nonvariable $\left(\log \left(P_{\mathrm{KS}}\right)>-2.0\right)$, in spite of their higher than average statistics, having between $\sim 40$ and 15000 counts, with a median of $\sim 111$ counts. A comparison with Fig. 5 shows that the variabile fraction of OB stars $(7.7 \%)$ is significantly lower than for the bulk of our sources with similar statistics. This finding agrees with the common view of X-ray emission from $\mathrm{O}$ stars, which is believed to be unrelated to solar-like magnetic activity, and rather is explained as the integrated emission from a large number of small shocks occurring in the strong winds of these stars (Feldmeier et al. 1997; Owocki \& Cohen 1999). Interestingly however, two of these sources, \#60 and \#979, are significantly variable, with $\log \left(P_{\mathrm{KS}}\right)$ values lower than -4 . Figure 7 shows their light-curves.

Source \#60 (Cyg\#12) is a B5 Ie star (Hanson 2003). The $\mathrm{X}$-ray light-curve shows a roughly linear decay of the ACIS count rate from $\approx 0.18 \mathrm{cnt} \mathrm{s}^{-1}$ to $\approx 0.16 \mathrm{cnt} \mathrm{s}^{-1}$. The star was observed by Waldron et al. (1998) who did not report variability during $\sim 125 \mathrm{ks}$ of non-continuous ROSAT PSPC observation. This kind of variability may be similar to the rotational modulation observed on the $\mathrm{O} 7 \mathrm{star} \theta$ Ori C (Gagné et al. 2005) and is attributed to the presence of a rigidly rotating magnetosphere (RRM), which may form in magnetic early-type stars with misaligned magnetic and rotation axe (Owocki et al. 2005). We defer further discussion of the nature of Cyg \#12 to Sect. 7.1.

Source \#979 is identified as star \#646 in Hanson (2003) and there is classified as a $\mathrm{B} 1.5 \mathrm{~V}+$ ? star, the question mark indicating the presence of an unresolved faint secondary. The observed variable X-ray emission is much fainter than in the previous case and might be due to the magnetic activity of the companion of the B1.5 V primary, presumably a lower mass star.

\section{Spectral analysis}

In order to characterize the hot plasma responsible for the X-ray emission of Cygnus OB2 stars, and to estimate their intrinsic X-ray luminosities, we analyzed the ACIS spectra of each of our 1003 sources. Reduced source and background spectra in the $0.5-8.0 \mathrm{keV}$ band were produced with AE (see Sect. 3.2), along with individual "redistribution matrices files" (RMF) and "ancillary response files" (ARF). For model fitting, spectra were grouped so to have a specified number of events in each energy bin. Grouping was tuned to the source statistics and we chose $2,5,7,10$, and 60 counts per channel for sources with netcounts in the following ranges: [0-40], [40-100], [100-200], [200-500], and [500-16000]. Spectral fitting of backgroundsubtracted spectra was performed with XSPEC v12.0 (Arnaud 2004) and our own shell and TCL scripts to automate the process as described in Flaccomio et al. (2006). Best fit parameters for the chosen models were found by $\chi^{2}$ minimization.

We fit our spectra assuming emission by a thermal plasma, in collisional ionization equilibrium, as modeled by the APEC code (Smith et al. 2001). Elemental abundances are not easily constrained with low-statistic spectra and were fixed at $Z=$ $0.3 Z_{\odot}$, with solar abundances taken from Anders \& Grevesse (1989). The choice of sub-solar abundances is suggested by several X-ray studies of star forming regions (e.g. Feigelson et al. 2002; Preibisch 2003). Absorption was accounted for using the WABS model, parametrized by the hydrogen column density, $N_{\mathrm{H}}$ (Morrison \& McCammon 1983).

Except for X-ray sources associated with O- and early B-type stars, which are discussed separately in Sect. 7.1, we fit source spectra with one-temperature (1T) plasma models using an automated procedure. In order to reduce the risk of finding a relative minimum in the $\chi^{2}$ spaces, our procedure chooses the best fit among several obtained starting from a grid of initial values of the model parameters: $\log \left(N_{\mathrm{H}}\right)=21.0,21.7,22.0,22.4$, 22.7 and $23.0 \mathrm{~cm}^{-2}$ and $k T=0.5,0.75,1.0,2.0,5.0 \mathrm{keV}$. Best fit values of $\log \left(N_{\mathrm{H}}\right)<20.8 \mathrm{~cm}^{-2}$ were truncated at 20.8 because, in the $0.5-8.0 \mathrm{keV}$ energy range, ACIS spectra are insensitive to lower column densities. For the same reason, 154 best fit values of $k T$ turned out to be $>10 \mathrm{keV}$ and were truncated at that value.

As noted by many authors (e.g. Getman et al. 2002, 2005b; Flaccomio et al. 2006), the spectral fitting of low statistic ACIS sources is problematic because of a degeneracy between plasma 

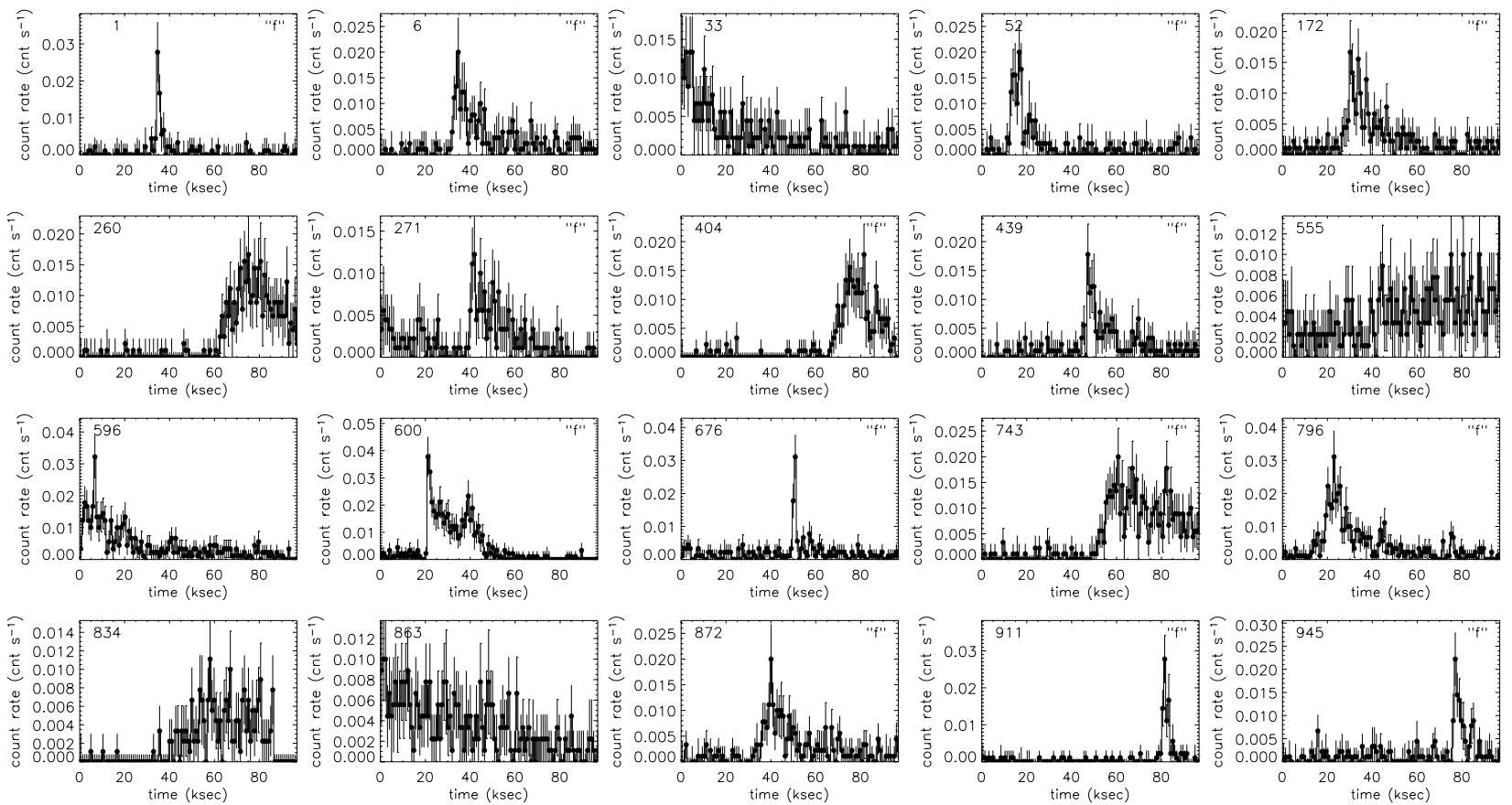

Fig. 6. Light curves (in the $0.5-8.0 \mathrm{keV}$ band) for 20 sources with variable emission during our $97.7 \mathrm{ks}$ Chandra observation. The bin length is $600 \mathrm{~s}$. Source numbers are given in the upper-left corner of each panel, followed by an " $\mathrm{f}$ " for sources classified as flaring.
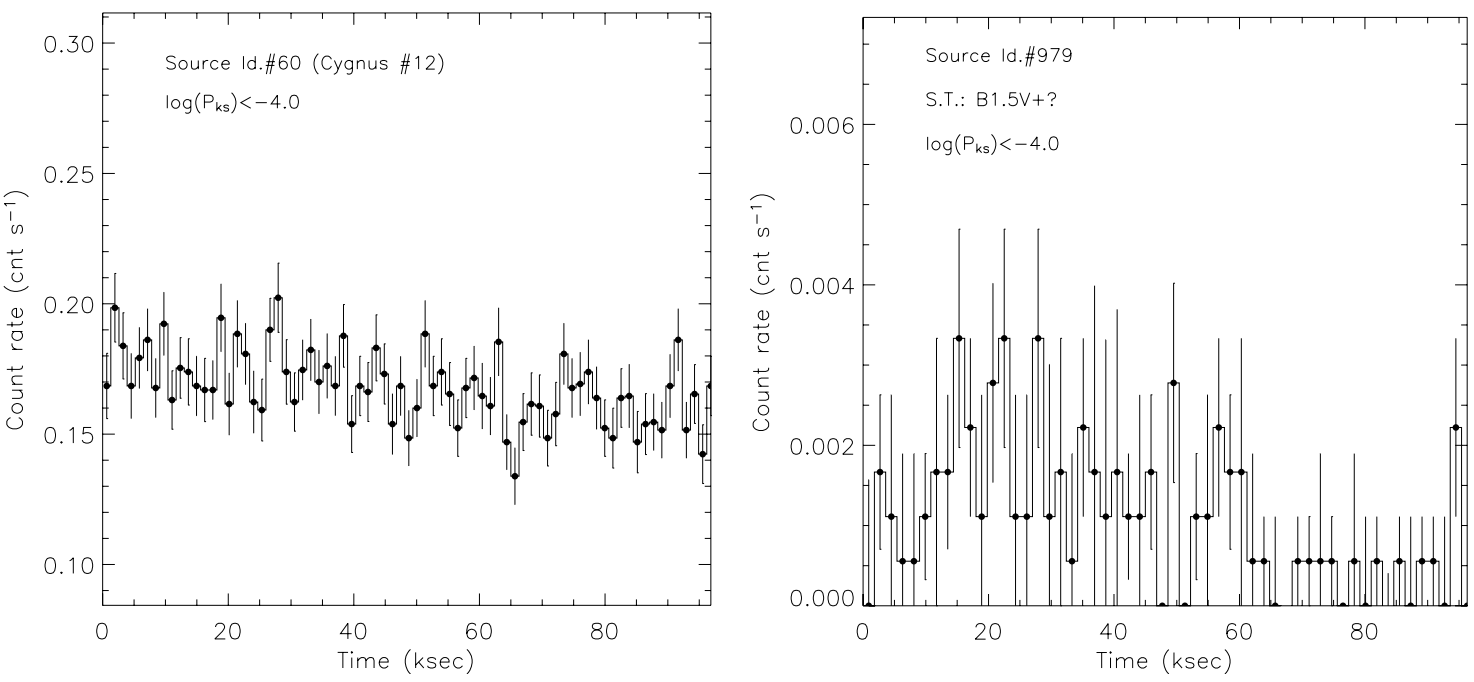

Fig. 7. Light curves in the $0.5-8.0 \mathrm{keV}$ band for the two variable early-type stars: Left: source Id. \#60, known as a B5Ie, has been recently classified a B5 $\pm 0.5 \mathrm{Ia}+$ star by Klochkova \& Chentsov (2004). X-ray variability of this star was unknown until now. The time bin-size is $900 \mathrm{~s}$. Right: source Id. \#979 is probably a B1.5 V+“"?” binary system (Massey \& Thompson 1991). The time bin size here is 1300 s. Source number and results of the KS test are given in the legend.

temperature and absorption. The degeneracy also results in a systematic bias: $k T$ values are often underestimated by as much as $\sim 50 \%$ while $N_{\mathrm{H}}$ values are overestimated. We investigated this issue with our data by considering the distributions of the best fit parameters for source in different count-statistic bins. Figure 8 shows the run of mean $k T$ and $N_{\mathrm{H}}$ with source counts for spectra fitted with 1T models. The systematic decrease of $N_{\mathrm{H}}$ and the increase of $k T$ with increasing source statistics are hardly explainable as physical effects and rather indicate that the spectral parameters obtained for sources with less than $\sim 20$ photons are ill-constrained.

The uncertainty on the $N_{\mathrm{H}}$ is particularly serious as it implies large and systematic uncertainties on the absorption-corrected $\mathrm{X}$-ray luminosities. In order to reduce the risk of erroneous results, we discarded from the following analysis results for source with $<20$ net photons, a total of 423 sources. Table 3 presents the results of the automated 1T spectral fits for 554 sources (the results of the spectral fitting for the 26 sources associated with known OB stars will be presented in Sect. 7.1, Table 4). We list source numbers (Col. 1) from Tables 1 and 2 , background subtracted counts in the spectra (2), the best fit hydrogen column densities and their $1 \sigma$ errors (3), the plasma temperatures and their $1 \sigma$ errors (4), and the emission measures (5). Columns (6) and (7) give the reduced $\chi_{v}^{2}$ for the spectral fits and the relative degrees of freedom, respectively.

The hydrogen column densities derived from X-ray spectral fitting depend of the interstellar material in the line of sight to the Cygnus OB2 cloud and on the location of stars within 


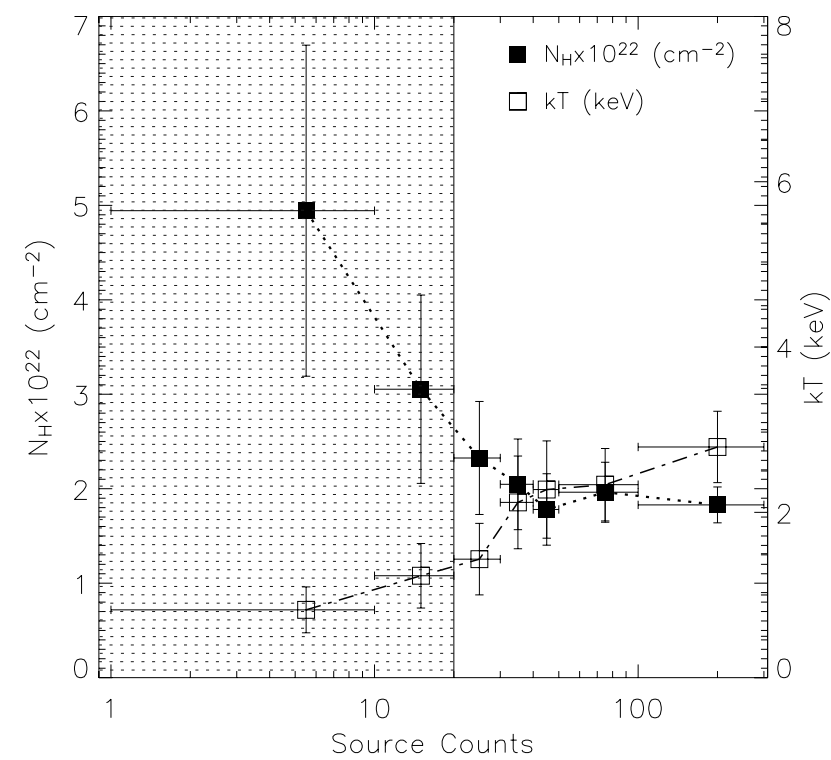

Fig. 8. Median $N_{\mathrm{H}}$ and $k T$ vs. source counts for sources fit with onetemperature models. Filled and empty squares indicate the average values of the $N_{\mathrm{H}}$ and $k T$ in each considered count range with vertical scales given on the right and left-hand side, respectively. The extent of the count ranges is indicated by the horizontal error bars. Vertical error bars indicate the median of the $1 \sigma$ uncertainties on the two parameters. For this plot we only considered $N_{\mathrm{H}}$ and $k T$ values with formal relative errors smaller than $90 \%$. The hatched area below 20 counts indicates the count-range in which spectral fits were discarded because of strong biases in the best fit parameters.

Table 3. Results of X-ray spectral fits: first rows. The complete version is available in the electronic edition of the Journal.

\begin{tabular}{|c|c|c|c|c|c|c|}
\hline $\begin{array}{l}N_{\mathrm{x}} \\
\# \\
\end{array}$ & $\begin{array}{l}\text { Cnts } \\
\text { (ph.) }\end{array}$ & $\begin{array}{l}\log \left(N_{\mathrm{H}}\right) \\
\left(\mathrm{cm}^{-2}\right)\end{array}$ & $\begin{array}{l}k T \\
(\mathrm{keV})\end{array}$ & $\begin{array}{l}\begin{array}{l}\log (\mathrm{EM}) \\
\left(\mathrm{cm}^{-3}\right)\end{array} \\
\end{array}$ & $\begin{array}{l}\text { Stat. } \\
\left(\chi_{v}^{2}\right)\end{array}$ & d.o.f. \\
\hline 1 & 67 & $22.13 \pm 0.26$ & 9.99 & 53.70 & 0.34 & 17 \\
\hline 2 & 72 & $21.05 \pm 0.34$ & $0.74 \pm 0.12$ & 53.40 & 1.54 & 17 \\
\hline 3 & 35 & $21.75 \pm 0.03$ & 9.99 & 53.40 & 0.38 & 9 \\
\hline 5 & 55 & $22.15 \pm 0.27$ & $6.05 \pm 8.52$ & 53.40 & 0.81 & 14 \\
\hline 6 & 216 & $22.13 \pm 0.68$ & $5.52 \pm 3.06$ & 54.10 & 0.96 & 19 \\
\hline 7 & 49 & $21.86 \pm 0.04$ & 9.99 & 53.40 & 0.78 & 15 \\
\hline 8 & 33 & $21.70 \pm 0.08$ & 9.99 & 53.40 & 0.51 & 9 \\
\hline 9 & 64 & $21.44 \pm 0.03$ & 9.99 & 53.40 & 0.76 & 14 \\
\hline 10 & 50 & $21.60 \pm 0.10$ & $1.04 \pm 0.23$ & 53.40 & 1.00 & 12 \\
\hline 12 & 99 & $22.14 \pm 0.47$ & $2.19 \pm 0.91$ & 53.88 & 1.02 & 24 \\
\hline 13 & 44 & $22.42 \pm 0.29$ & $3.74 \pm 3.87$ & 53.70 & 1.20 & 10 \\
\hline 14 & 75 & $22.51 \pm 0.30$ & 9.99 & 53.70 & 1.10 & 19 \\
\hline 15 & 60 & $21.90 \pm 0.24$ & $6.85 \pm 8.19$ & 53.40 & 0.66 & 14 \\
\hline 16 & 30 & $22.53 \pm 0.10$ & $2.72 \pm 2.67$ & 53.70 & 0.82 & 7 \\
\hline 17 & 3173 & $22.16 \pm 0.25$ & $1.34 \pm 0.64$ & 55.65 & 1.57 & 252 \\
\hline 19 & 25 & $22.48 \pm 0.15$ & $3.43 \pm 4.19$ & 53.40 & 0.56 & 6 \\
\hline 20 & 49 & $22.26 \pm 0.30$ & $3.04 \pm 1.86$ & 53.70 & 0.72 & 11 \\
\hline
\end{tabular}

the cloud material of Cygnus OB2. Figure 9a shows the distribution of $\log N_{\mathrm{H}}$ values for the 580 sources with more than 20 counts. They appear to be normally distributed with a median $\log N_{\mathrm{H}} \sim 22.25\left(\mathrm{~cm}^{-2}\right)$ and a $F W H M$ of $\sim 0.21$ dex. The shaded area in Fig. 9a indicates an apparent excess of relatively unabsorbed sources $\left(\log N_{\mathrm{H}}<21.8 \mathrm{~cm}^{-2}\right)$ with respect to the lognormal distribution of the bulk of the sources. These $\sim 23$ sources are likely associated with foreground stars. Most of these sources seem to be spatially distributed uniformly in the FOV of the Cygnus OB2 region.
Another interesting observation is that the $N_{\mathrm{H}}$ distribution of the $106 \mathrm{X}$-ray sources without near-IR counterparts (dark gray histogram in Fig. 9) seems to be skewed toward higher values than the global distribution. For example, while only $11 \%$ of the $\mathrm{X}$-ray sources with counterparts have $N_{\mathrm{H}}>22.5$, this is true for $29(27 \%)$ of the unidentified sources.

According to the relationship between $N_{\mathrm{H}}$ and $A_{\mathrm{V}}, N_{\mathrm{H}}=$ $2.2 \times 10^{21} A_{\mathrm{v}} \quad \mathrm{cm}^{-2}$ (Ryter 1996), the median $N_{\mathrm{H}}$ of all detected sources with more than 20 counts corresponds to $A_{\mathrm{v}} \sim$ $8.1_{5.1}^{12.8} \mathrm{mag}$. If we only consider the sources with near-IR counterparts (and $>$ than 20 counts) we obtain $\log N_{\mathrm{H}}=22.23$, which corresponds to $A_{\mathrm{v}}=7.7 \mathrm{mag}$, i.e. in reasonable agreement with the value calculated from the $\operatorname{NIR~CMD~}\left(A_{\mathrm{v}} \approx 7.0 \mathrm{mag}\right)$.

Figure 9(b) shows the distribution of plasma temperatures: it peaks at $\sim 1.35 \mathrm{keV}$, has a median $\sim 2.4 \mathrm{keV}$ and shows an extended hard tail. Variable sources $\left(\log P_{\mathrm{KS}}<-2\right)$ have harder spectra with median $k T=3.3 \mathrm{keV}$ and flaring sources (see hatched histogram in Fig. 9b) are even harder with a median $k T=3.75 \mathrm{keV}$.

We derived unabsorbed X-ray luminosities for each of our sources. For $\mathrm{O}$ and B-type stars, discussed in detail below (Sect. 7.1), luminosities were derived from individual spectral fits. For the other sources, given the considerable uncertainties in the absorption estimates, especially at the faint end, we preferred not to use the results of spectral fits individually. Rather, we computed a single count-rate to $L_{\mathrm{X}}$ conversion factor that takes into consideration the average intrinsic source spectrum and interstellar absorption. This conversion factor, computed as the median ratio between the individual unabsorbed X-ray luminosities (from the best fit spectral models) and the source countrates is $7.92 \times 10^{33} \mathrm{ergs}$.

\section{Results}

We now discuss the implications of our data for the understanding of X-ray emission from young stars and for the study of the Cygnus OB2 stellar population. We split the discussion according to stellar mass and, correspondingly, expected X-ray emission mechanism. We first discuss known $\mathrm{O}$ and early $\mathrm{B}(<\mathrm{B} 2)$ stars, i.e. massive stars $\left(M \gtrsim 12 M_{\odot}\right)$ expected to generate $\mathrm{X}$-rays in their powerful stellar winds. Next, using our mass estimates based on the $J$-band magnitude (Sect. 4.2), we define and study the properties of two subsamples of intermediate and lowmass X-ray detected stars. We base the distinction between the two groups on the model-predicted thickness of the convective layer, whose presence has been often found to be correlated with solar-like coronal activity. Stars with small or absent convective layers are instead believed to be unable to sustain coronal activity, although the matter is somewhat controversial. Adopting the 2 Myr isochrone from the SDF models, we observe that the thickness of the convective layer relative to the stellar radius, $R_{\mathrm{c}}$, decreases rapidly, from $\sim 5 \%$ to $<1 \%$, as the stellar mass increases from 3 to $3.3 M_{\odot}$. This rather sharp boundary however lies in the 2.8-5.3 $M_{\odot}$ range in which our mass estimates are degenerate and therefore unconstrained (see Sect. 4.2). In order to resolve this ambiguity, we define the intermediate-mass range as $2.8-10 M_{\odot}$, resulting in the selection of $57 \operatorname{stars}^{17}$. Low mass stars are finally defined as stars with $M<2.8 M_{\odot}$ and are

\footnotetext{
17 There are only 28 stars with masses in the $2.8-5.3 M_{\odot}$ range and for which this interval formally corresponds to the uncertainty on the mass estimate. We therefore expect a small fraction of the 57 intermediate mass stars to lie in the $2.8-3.0 M_{\odot}$ range and to have a substantial model-predicted convective envelope.
} 
Table 4. X-ray spectral parameters of the Cygnus OB2 OB-type stars.

\begin{tabular}{|c|c|c|c|c|c|c|c|c|c|c|}
\hline \multirow{2}{*}{$\begin{array}{l}\text { Src. } \\
\#\end{array}$} & \multicolumn{2}{|c|}{ " Optical Parameters } & \multirow[t]{2}{*}{$\chi_{v}^{2}$} & \multirow{2}{*}{$\begin{array}{l}\text { Pile-up } \\
(\%)\end{array}$} & \multicolumn{2}{|c|}{$N_{\mathrm{H}}\left[\times 10^{22}\right]\left[\mathrm{cm}^{-2}\right]$} & \multirow{2}{*}{$\begin{array}{l}k T_{1} \\
{[\mathrm{keV}]}\end{array}$} & \multirow{2}{*}{$\begin{array}{l}k T_{2} \\
{[\mathrm{keV}]}\end{array}$} & \multirow{2}{*}{$\begin{array}{l}\text { Abundance } \\
{\left[Z_{\odot}\right]}\end{array}$} & \multirow{2}{*}{$\begin{array}{l}\text { Flux }\left[10^{-13}\right] \\
{\left[\mathrm{erg} \mathrm{s}^{-1} \mathrm{~cm}^{-2}\right]}\end{array}$} \\
\hline & Hanson\# & S.T. & & & WABS & ABSORI & & & & \\
\hline $60^{b}$ & 304 & B5 Ie & 1.4 & $\sim 18$ & $2.15 \pm 0.15$ & $0.87 \pm 0.12$ & $0.63 \pm 0.03$ & $1.56 \pm 0.11$ & $0.43 \pm 0.04$ & $235.4_{2017}^{255.4}$ \\
\hline 133 & 339 & $08.5 \mathrm{~V}$ & 1.1 & no & $0.24 \pm 0.18$ & - & $1.53 \pm 0.68$ & - & $(0.3)$ & $0.11_{0.07}^{0.19}$ \\
\hline 253 & 378 & B0 V & 1.0 & no & $3.57 \pm 0.09$ & - & $0.43 \pm 0.15$ & - & $(0.3)$ & $2.00_{142}^{2.21}$ \\
\hline 315 & 390 & $\mathrm{O} 8 \mathrm{~V}$ & 0.8 & no & $3.30 \pm 0.08$ & - & $0.44 \pm 0.10$ & - & $0.15 \pm 0.09$ & $0.64_{0.51}^{0.73}$ \\
\hline 433 & 417 & O4 III(f) & 1.5 & $\lesssim 2$ & $2.30 \pm 0.07$ & - & $0.39 \pm 0.06$ & $1.94 \pm 0.73$ & $0.60 \pm 0.34$ & $42.1_{34.6}^{0.31}$ \\
\hline 456 & 421 & $09.5 \mathrm{~V}$ & 1.4 & no & $1.45 \pm 0.08$ & - & $1.71 \pm 1.02$ & - & $(0.30)$ & $1.06_{1.09}^{0.88}$ \\
\hline $488^{b}$ & 431 & O5 If & 1.3 & $\sim 12$ & $2.09 \pm 0.07$ & - & $0.79 \pm 0.05$ & $2.82 \pm 0.61$ & $0.65 \pm 0.10$ & $72.6_{67.5}^{1.59}$ \\
\hline 530 & 448 & $\mathrm{O} 6 \mathrm{~V}$ & 1.4 & no & $1.97 \pm 0.34$ & - & $0.65 \pm 0.20$ & - & $(0.1)$ & $1.18_{0.96}^{1.39}$ \\
\hline 537 & 455 & $\mathrm{O} 8 \mathrm{~V}$ & 1.1 & no & $2.19 \pm 0.07$ & - & $0.61 \pm 0.17$ & - & $(0.1)$ & $1.20_{1.07}^{1.28}$ \\
\hline 544 & 457 & O3 If & 1.3 & $\lesssim 2$ & $2.12 \pm 0.06$ & $0.56 \pm 0.09$ & $0.39 \pm 0.14$ & $1.32 \pm 0.19$ & $0.97 \pm 0.3$ & $88.8_{77.5}^{98.6}$ \\
\hline 562 & 462 & O6.5 II & 1.0 & no & $2.52 \pm 0.48$ & - & $0.40 \pm 0.12$ & - & $0.14 \pm 0.1$ & $8.45_{64}^{9.1}$ \\
\hline $568^{a}$ & 465 & O6If + O5.5III(f) & 1.4 & $\sim 15$ & $2.94 \pm 1.25$ & - & $0.91 \pm 0.05$ & $2.64 \pm 0.07$ & $0.52 \pm 0.07$ & $61.3_{57.9}^{04.9}$ \\
\hline 584 & 470 & $09.5 \mathrm{~V}$ & 0.8 & no & $1.85 \pm 0.07$ & - & $0.44 \pm 0.18$ & - & $(0.3)$ & $3.49_{2.89}^{3.98}$ \\
\hline 597 & 473 & $08.5 \mathrm{~V}$ & 1.0 & no & $1.88 \pm 0.09$ & - & $0.30 \pm 0.22$ & - & 1.07 & $3.00_{238}^{3.78}$ \\
\hline 615 & 480 & $07.5 \mathrm{~V}$ & 1.0 & no & $2.73 \pm 0.06$ & - & $0.50 \pm 0.17$ & - & $(0.1)$ & $4.01_{3.42}^{4.43}$ \\
\hline 625 & 483 & O5 If & 1.0 & no & $1.00 \pm 0.08$ & - & $0.55 \pm 0.06$ & - & $0.60 \pm 0.21$ & $99.4_{86.4}^{107.3}$ \\
\hline 626 & 485 & $\mathrm{O} 8 \mathrm{~V}$ & 1.1 & no & $1.65 \pm 0.04$ & - & $0.88 \pm 0.23$ & - & $(0.1)$ & $0.38^{0.53}$ \\
\hline 686 & 507 & $08.5 \mathrm{~V}$ & 1.1 & no & $2.39 \pm 0.08$ & - & $0.35 \pm 0.11$ & - & $(0.3)$ & $4.16_{3.28}^{5.021}$ \\
\hline $729^{a}$ & 516 & $05.5 \mathrm{~V}(\mathrm{f})$ & 1.1 & $\lesssim 4$ & $0.81 \pm 0.05$ & $0.51 \pm 0.32$ & $1.98 \pm 0.12$ & - & $0.38 \pm 0.09$ & $11.7_{10.1}^{3.28}$ \\
\hline 779 & 534 & $07.5 \mathrm{~V}$ & 1.2 & no & $1.16 \pm 0.03$ & - & $2.28 \pm 0.55$ & - & $(0.1)$ & $6.05_{4.91}^{7.74}$ \\
\hline 817 & 556 & $\mathrm{~B} 1 \mathrm{Ib}$ & 0.9 & no & $1.57 \pm 0.08$ & - & $0.18 \pm 0.07$ & - & $(0.3)$ & $1.20_{0.94}^{4.53}$ \\
\hline 881 & 588 & B0 V & 0.9 & no & $1.84 \pm 0.05$ & - & $0.42 \pm 0.20$ & - & (0.3) & $0.81_{0.71}^{0.94}$ \\
\hline 895 & 601 & O9.5 III & 1.0 & no & $1.98 \pm 0.07$ & - & $0.38 \pm 0.21$ & - & $(0.3)$ & $0.72_{050}^{0.92}$ \\
\hline 971 & 642 & B1 III & 0.9 & no & $1.73 \pm 0.05$ & - & $0.96 \pm 0.45$ & - & (0.3) & $0.14_{0.09}^{0.30}$ \\
\hline 979 & 646 & $\mathrm{~B} 1.5 \mathrm{~V}+?$ & 1.5 & no & $2.90 \pm 1.24$ & - & $1.92 \pm 1.01$ & - & (0.3) & $0.22_{0.29}^{0.09}$ \\
\hline 1001 & 696 & $09.5 \mathrm{~V}$ & 0.8 & no & $0.71 \pm 0.02$ & - & $0.54 \pm 0.17$ & - & (0.3) & $0.71_{0.42}^{0.83}$ \\
\hline
\end{tabular}

Notes: (1) Abundance values in parenthesis indicate cases for which abundances were fixed in the spectral fit (see text), (2) Absorption-corrected fluxes are calculated in the $0.5-8.0 \mathrm{keV}$ energy range (3) Three bright sources, \#60, \#488, and \#568, suffer pile-up, and we extracted the spectra from annular rings (see Sect. 3.2) that exclude the central parts of the PSFs. ${ }^{a}$ The $6.4+6.7 \mathrm{keV} \mathrm{FeK}{ }_{\alpha}$ complex is observed; ${ }^{b}$ The FeI $6.4 \mathrm{keV}$ line is not detected, but the $6.7 \mathrm{keV}$ seems to be present.
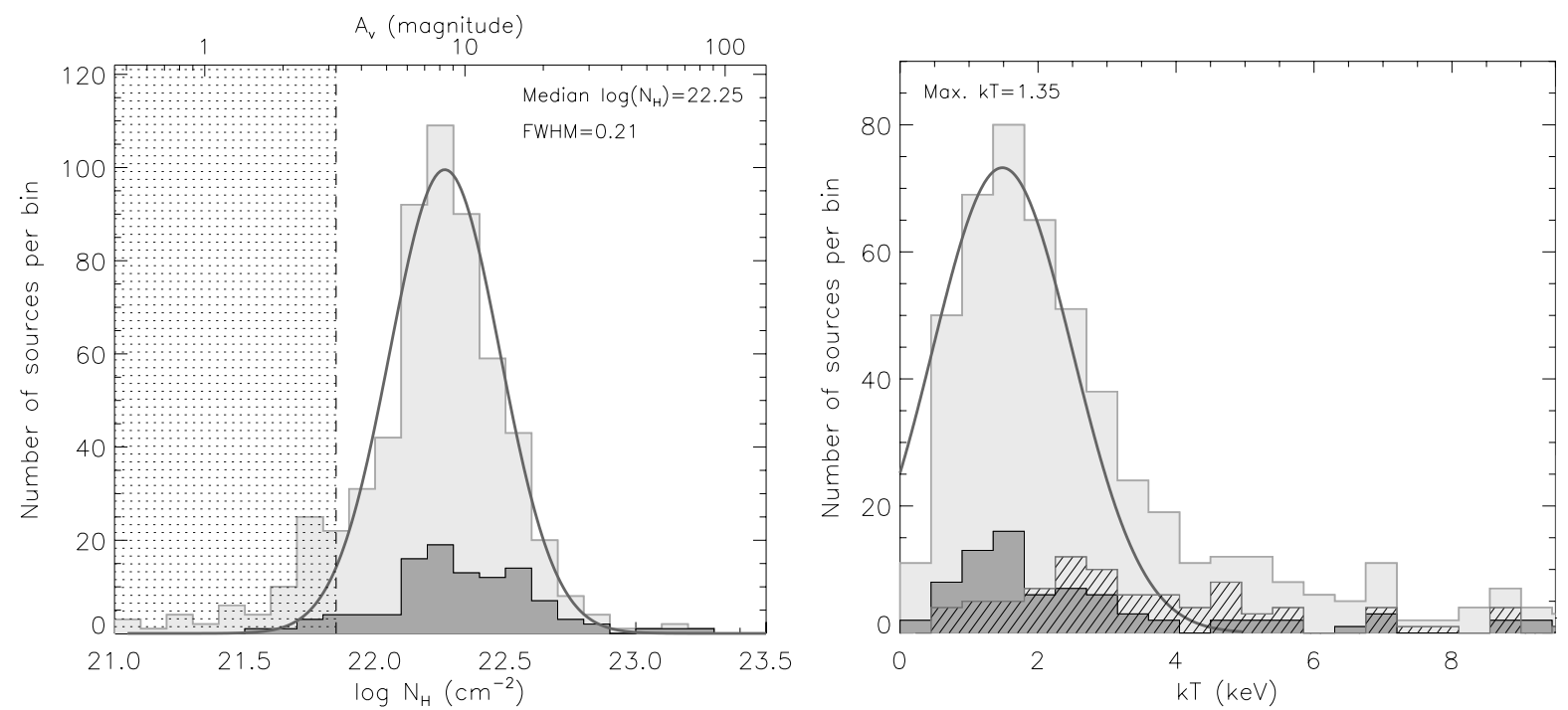

Fig. 9. a) Distributions of absorbing columns, $N_{\mathrm{H}}$, with the corresponding visual absorption scale at the top. The light gray area refers to sources with net-counts greater than 20. The maximum of the distribution is at $\log N_{\mathrm{H}} \sim 22.25 \mathrm{~cm}^{-2}$ with a Full Width Half Maximum (FWHM) of about 0.21 dex. The shaded area on the left indicates an excess of low-absorption sources with respect to the log-normal distribution that best describes the histogram (the continuous curve), possibly associated with foreground stars. The darker gray histogram refers to the subsample of sources without a MASS counterpart. b) Same as panel a) for plasma temperatures $(k T)$ with, in addition, the distribution for "flaring" sources (hatched histogram). The peak of the overall distribution is at $\sim 1.35 \mathrm{keV}$. 


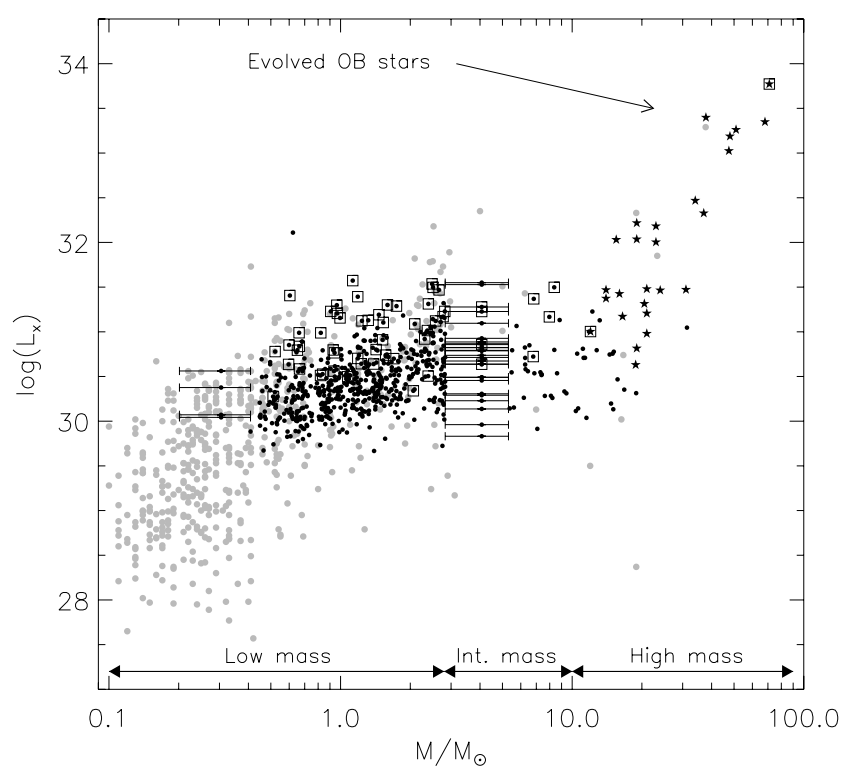

Fig. 10. $\log M / M_{\odot}$ vs. $\log L_{\mathrm{x}}$ for Cygnus OB2 sources with NIR counterparts (black circles and star-symbols) and for ONC sources observed by the COUP project (gray circles). Variable sources are indicated by empty squares, known O- and early B-type stars by star-symbols.

predicted to have substantial convective envelopes $\left(R_{\mathrm{c}}>18 \%\right)$. 578 counterparts to X-ray sources fall in this category. Because of the sensitivity limit of 2MASS only 25(4) sources of these stars have masses below $0.5(0.4) M_{\odot}$, which we may consider as our approximate detection limit. Our completeness limit is however probably higher, at $\sim 1 M_{\odot}$, corresponding to $J=15.8$, i.e. roughly the completeness limit of $2 \mathrm{MASS}$. A histogram of the logarithm of stellar masses also peaks at $\sim 1 M_{\odot}$, confirming the above estimate given that the mass function usually increases towards lower masses.

Figure 10 shows the $L_{X}$-mass scatter plot for $683 \mathrm{X}$-ray sources in all three mass ranges. We distinguish with different symbols stars of low- and intermediate-mass, as determined from the $J$-band magnitude, and massive stars with known $\mathrm{O}$ and early B- spectral types ${ }^{18}$. Note that stars in the $2.8-5.3 M_{\odot}$ and $0.2-0.4 M_{\odot}$ ranges are plotted with horizontal error bars spanning the entire range, because the $J$-band vs. mass relation was found to be degenerate. In the plot we also indicate the position of 57 time-variable X-ray sources. They appear to be brighter than other sources of the same mass, probably because variability is more readily detected in sources with high photon statistics (see Sect. 5).

\subsection{High-mass stars}

The Cygnus OB2 region is one of the most massive SFR in the Galaxy, with $\sim 2600 \pm 400$ OB star members (Knödlseder 2000). The presence of a variety of evolved stars suggests that the star formation process was non-coeval (Massey \& Thompson 1991; Hanson 2003). Out of a total of 20 O-type and 13 B-type stars lying in our Cygnus OB2 ACIS FOV, 7 and 3, respectively, are evolved (luminosity class: I-III). While we detect X-ray emission from all $\mathrm{O}$ stars regardless of evolutionary status, we have only detected 6 B stars, among which all the evolved ones. The

\footnotetext{
18 A number of X-ray detected stars with no spectral type appear to be of high mass but with lower X-ray luminosities than those with spectral types. In the discussion of high mass stars, however, we will only consider stars with spectral types.
}

detection fraction of evolved B stars is thus $100 \%$ while it is only $33 \%$ ( 3 our of $9^{19}$ ) for un-evolved ones.

The most widely accepted explanation for the X-ray emission of single $\mathrm{O}$ and early B-type stars invokes multiple smallscale shocks in the inner layers of their radiation-driven stellar winds (e.g. Feldmeier et al. 1997). Recent theoretical and observational results however support, for several OB stars, another physical plasma heating model involving strong magnetic fields: the magnetically channeled wind shock (MCWS) model (Schulz et al. 2003; Owocki et al. 2005; Gagné et al. 2005). Moreover, binary systems in which both components are O- and early B-type stars can produce intense thermal X-ray emission from windwind interactions as well as non-thermal X-ray emission from Inverse Compton scattering.

We modeled the spectra of $\mathrm{O}$ and $\mathrm{B}$ stars with one- or two-temperature thermal plasma emission models (APEC), absorbed by neutral interstellar and circumstellar material (WABS). Because the dense stellar winds of evolved OB stars are affected by the strong stellar UV/EUV ionizing radiation, the additional absorption of X-rays by a partially ionized stellar also wind should be considered. For evolved stars we then decided to model the combined effect of ISM plus wind material with a warm absorption model (WABS $\times$ ABSORI, see e.g. Waldron et al. 1998). For sources \#60, \#544, and \#729, we found that the inclusion of a warm absorber in the spectral model yields satisfactory fits of the soft part of the spectra $(\leq 1.2 \mathrm{keV})$, reducing the $\chi_{v}^{2}$ from 2.1 to 1.4 , from 2.7 to 1.3 , and from 2.2 to 1.2 , for the three sources respectively. The spectra of other evolved sources did not support the presence of the warm absorber, although in many cases the spectra have too low statistics to be conclusive. Metal abundances were fixed at $Z=0.3 Z_{\odot}$ in fitting faint sources $(<100 \mathrm{ph}$.) and left as a free parameter for brighter sources. When the best fit abundance was lower than $Z=0.1 Z_{\odot}$ we repeated the fit with $Z$ fixed at this value.

Figure 12 shows three notable examples of spectral modeling and the best-fit parameter values for the 26 detected OB stars are presented in Table 4. We list: X-ray source numbers from Table 1 (Col. 1), identification numbers and spectral types from Hanson (2003) ( 2 and 3), reduced $\chi^{2}$ of the best fit (4), fraction of pile-up as reported by AE (as discussed Sect. 3.2, if $\mathrm{f}$ (Pile-up) $>2 \%$ we used an annular photon extraction region that excludes the peak of the PSF), the $N_{\mathrm{H}}$ of the cold and hot absorption components from WABS and ABSORI, respectively (6 and 7), plasma temperatures of the two components (8 and 9), metal abundances (10). Column (11) finally gives the un-absorbed X-ray flux in the $0.5-8.0 \mathrm{keV}$ energy band.

The median absorption of the $26 \mathrm{OB}$ stars is $\log N_{\mathrm{H}}=$ $22.19 \mathrm{~cm}^{-2}$, very similar but slightly lower than the median value of lower mass stars (22.23, see Sect. 7.3). It is tempting to attribute the lower absorption of $\mathrm{O}$ stars to the sweeping of the cloud material by strong winds. A two-sided KS test comparing the distribution of $N_{\mathrm{H}}$ for $\mathrm{O}$ and B stars with that of lower mass ones gives, however, a null result.

Most of the O- and early B- type stars are well fit by a soft thermal emission model with $k T$ ranging between 0.5 and $0.7 \mathrm{keV}(\sim 5.8-8.1 \mathrm{MK})$, in agreement with the predictions of the wind shock model (Lucy \& White 1980; Owocki et al. 1988). The spectra of some sources, however, require a second (hard) thermal component, which is not predicted by the wind shock model. The presence of this component seems to depend on the stellar evolutionary status: out of the 10 evolved O- and early B-type stars (spectral types B5 Ie,

\footnotetext{
${ }_{19}$ One undetected B stars has an unknown luminosity class.
} 
O4 III, O5 If, O3 If, O6 If +O5.5III(f)) 5 (50\%) show evidence of a hard thermal emission with $k T$ roughly between 1.3 and $3.0 \mathrm{keV}(14-35 \mathrm{MK})$, while among the 16 class $\mathrm{V}$ stars, the hot component is only required in 3 cases (i.e. 23\%). Several physical mechanisms have been proposed to explain the hard emission observed in some of our OB stars:

- Magnetically Confined Wind Shocks (MCWS). X-ray emission from MCWS is predicted by simulations of radiationdriven winds in the presence of a magnetic field (ud-Doula \& Owocki 2002) and it has most likely been observed in O-type stars (e.g. $\theta^{1}$ Orionis C, Gagné et al. 2005). The model predicts that the overall degree of plasma confinement is determined by a single dimensionless parameter $\eta_{\star} \equiv \frac{B_{\mathrm{eq}}^{2} R_{\star}}{\dot{M} v_{\infty}}$, where $B_{\text {eq }}$ is the equatorial dipole magnetic intensity at the stellar surface, $R_{\star}$ is the stellar radius, $\dot{M}$ is the mass-loss rate, and $v_{\infty}$ is the terminal wind speed. Simulations with typical parameters for OB stars predict high post-shock temperatures of about 20-30 MK (1.7-2.6 keV). Furthermore, in the case of oblique rotators, i.e. when the rotational and magnetic axes are misaligned, rotational modulation of the X-ray emission can be observed. This might be the case of our source B5 Ie (Id.\#60 $\equiv$ Cyg\#12) for which $k T_{2}=1.56 \mathrm{keV}$ and the light-curve shows, during our observation, a gradual decay (cf. Figs. 7 and 12).

- Highly compressed wind shocks in evolved O-type stars or colliding winds in the case of early-type binary systems (Stevens et al. 1992; Raassen et al. 2003). Our source \#568 (Cyg\#8) is an O6If + O5.5III(f) supergiant-giant pair, whose $\mathrm{X}$-ray spectrum indicates hot thermal plasma at $k T_{2}=2.64 \pm$ $0.07 \mathrm{keV}$. A colliding wind scenario is moreover suggested by the presence of the $\mathrm{FeK}_{\alpha}$ complex $(6.4+6.7 \mathrm{keV})$ in its spectrum ${ }^{20}$ (cf. Fig. 12). The other two O-type supergiants, sources \#544 (O3 If) and \#625 (O5 If, see Fig. 12), although requiring a second thermal component, show rather soft spectra and no $\mathrm{FeK}_{\alpha}(6.4+6.7 \mathrm{keV})$ complex.

- Inverse Compton scattering of photospheric UV photons by relativistic particles that are Fermi-accelerated in shocks within the chaotic stellar winds of OB supergiants (cf. Chen $\&$ White 1991). The actual production of the needed relativistic electrons by stellar wind shocks is, however, still in doubt. We can likely rule out this possibility for our Cygnus OB2 OB stars, given that all of them are well fit by thermal emission models in which the lines of several elements give a significant contribution.

One of the most intriguing and so far unexplained observational results regarding the X-ray emission of O- and early B-type stars is the existence of a simple proportionality between the X-ray luminosity $\left(L_{\mathrm{x}}\right)$, and the bolometric luminosity $\left(L_{\mathrm{bol}}\right)$ (Berghoefer et al. 1997). We explore this relationship for Cygnus OB2 Oand early B-type stars in Fig. 11. $L_{\mathrm{x}}$ values were calculated from our X-ray spectral analysis. Bolometric luminosities with relative uncertainties were estimated form the known spectral types and the calibrations given by Martins et al. (2005).

20 The temperatures reached by hydrodynamic shocks in the winds of single stars are usually not high enough to produce significant $\mathrm{Fe}_{\alpha}$ emission. In wide early-type binary systems, however, stellar winds collide with velocities close to $v_{\infty}$ and are thus heated to sufficiently high temperatures. In such cases, the cooler surrounding wind material, excited by the high energy radiation, can produce the $6.4 \mathrm{keV}$ fluorescent Fe line. The observation of the Fe-complex can thus be a used as diagnostic for colliding-wind binaries (Raassen et al. 2003).

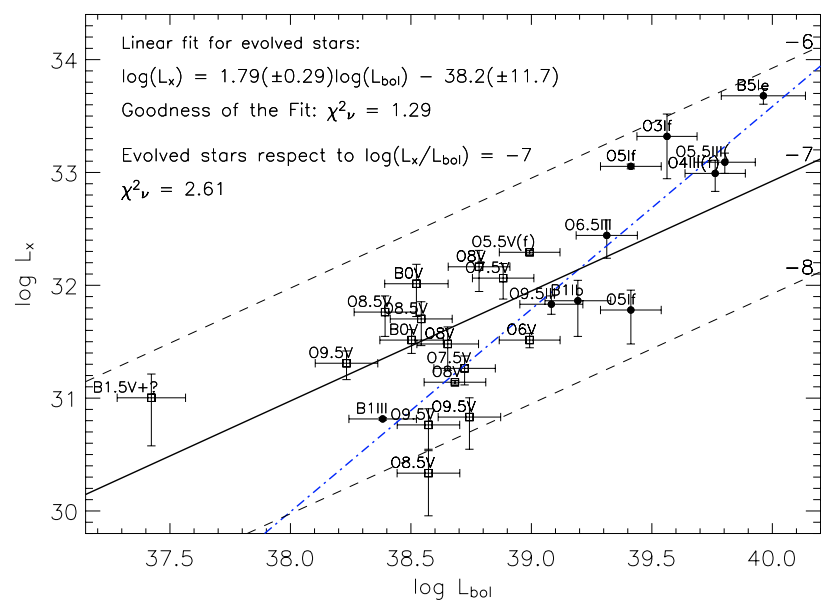

Fig. 11. X-ray versus bolometric luminosity for main-sequence and evolved OB-type stars (open and filled symbols, respectively) in the Cygnus OB2 region. The dotted and continuous lines indicate $\log L_{\mathrm{x}} / L_{\mathrm{bol}}=-6,-7$, and -8 . Evolved stars (i.e. giants and supergiants) seems to follow a power-law relation with index $\sim 1.8$ (dashed line).

The proportionality between $L_{\mathrm{x}}$ and $L_{\mathrm{bol}}$ is retrieved, albeit with a large dispersion. However, when we distinguish between main sequence stars and evolved ones (i.e. giants and supergiants), we discover, for these latter, an apparently tight $L_{\mathrm{x}}-L_{\mathrm{bol}}$ relationship which is not a simple proportionality. For evolved stars, the $\chi_{v}^{2}$ with respect to the $L_{\mathrm{X}}=10^{-7} L_{\text {bol }}$ relation is 2.61 , while if we adopt the best-fit power law (with index $\Gamma \sim 1.8$ ) the $\chi_{v}^{2}$ is reduced to $\sim 1.3$. We test the statistical significance of including this additional degree of freedom in the $L_{x} / L_{\text {bol }}$ relation. We used a $F_{X}$-test as described in Bevington et al. 1969. We found that $\mathrm{F}_{\chi}$-test $\sim 0.6$, which does not indicate a significant improvement of the quality of the fit $\left(\mathrm{F}_{\chi}\right.$ should be greater than 1.1), even when adopting a significance level as high as 0.1 . However, this result is affected by the small number of evolved $\mathrm{O}$ and $\mathrm{B}$ stars in our sample. A similar analysis with more stars from other regions will be needed to confirm, or disprove, this tentative result.

A more detailed study of the physical origins of the X-ray emission from OB stars and on its dependence on stellar wind parameters will be presented in forthcoming paper.

\subsubsection{Specific sources}

We now discuss the results of our analysis for three noteworthy OB stars in the ACIS FOV that have been the subject of previous investigations.

- Cygnus OB2 \#8 (ACIS source \# 568). It has recently been classified as an O6If + O5.5III(f) binary with a $21.9 \mathrm{~d}$ period and strong evidence of phase-locked X-ray variability (De Becker \& Rauw 2005). Our observation only covers $\sim 5 \%$ of the orbital period and we do not detect variability. Cygnus OB2 \#8 has also been observed by Waldron et al. (2004) with the Chandra High Energy transmission Grating Spectrometer (HETGS). They fit the X-ray spectrum with a a $2 \mathrm{~T}$ thermal model with $T_{1}=3.98 \pm 1.67 \mathrm{MK}$ $(0.34 \pm 0.14 \mathrm{keV})$ and $T_{2} 13.88 \pm 1.80 \mathrm{MK}(1.2 \pm 0.15 \mathrm{keV})$ and obtain an $L_{\mathrm{x}} / L_{\text {bol }}$ ratio of $\sim 1.8 \times 10^{-7}$. The spectral parameters are in rough agreement with those obtained here (see Fig. 12 and results presented in Table 4), but we find a somewhat higher $L_{\mathrm{x}} / L_{\mathrm{bol}}, \sim 4.0 \times 10^{-7}$. The discrepancy is 

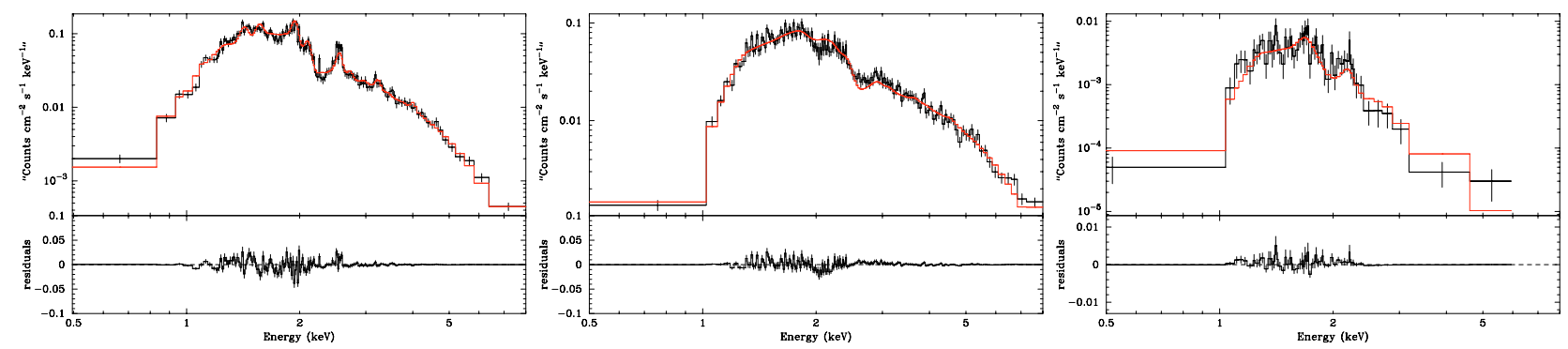

Fig. 12. ACIS-I spectra of three OB stars. The data were fitted with absorbed two temperatures optically thin thermal plasma models. Left: Cyg\#12 (Src.Id. \#60), showing a prominent FeK $6.7 \mathrm{keV}$ emission line, indicative of hot thermal plasma. Center: Cyg\#8 (Src.Id. \#568), a colliding wind binary with a strong $\mathrm{FeK}_{\alpha}$ blend at $6.4+6.7 \mathrm{keV}$, arguing in favor of the thermal nature of the hard part of the spectrum and consistent with the expected emission from a colliding wind region (Raassen et al. 2003). Right: source \#625 (O5.5 If), a typical super-giant fit with a 1-T model with a soft temperature model: $K T_{1}=0.55 \pm 0.06 \mathrm{keV}$.

however due exclusively to the different adopted bolometric luminosities: Waldron et al. (2004) use $\log L_{\mathrm{bol}}=39.79$ from Bieging et al. (1989), while here we adopt $\log \left(L_{\mathrm{bol}}\right)=39.4$ obtained from Herrero et al. (2002).

- Cygnus OB2 \#9 (ACIS source \# 488). An O5 If super giant, it is the strongest and most variable non-thermal radio emitter in Cygnus OB2 (Van Loo 2005). In X-rays it is however fainter than other evolved stars. Cygnus OB2 \#9 has not been indicated as a binary in the literature. However, periodic radio variability has been observed with a period of $\sim 2.35 \mathrm{yr}$ (Blomme, private communication). Waldron et al. (1998) did not detect variability in EINSTEIN and ROSAT X-ray data. Rauw et al. (2005) successfully fit the XMMNewton EPIC X-ray spectrum with an absorbed 2-T thermal model $\left(k T_{1}=0.63 \pm 0.03\right.$ and $\left.k T_{2} \sim 2.4 \mathrm{keV}\right)$. These results are roughly consistent with ours (cf. Table 4).

- Cygnus OB2 \#12 (ACIS source \# 60) has been variously classified and its spectral type is probably variable. Most investigations however agree with an extremely luminous B supergiant B5Ie (Hanson 2003), possibly related to the Luminous Blue Variable (LBV) phenomenon. Twodimensional spectral classification suggests a $\mathrm{B} 5+/-0.5 \mathrm{Ia}^{+}$ spectral type (Klochkova \& Chentsov 2004). In our near-IR color-color diagram (Fig. 3), Cygnus OB2 \#12 appears to be highly absorbed and to be affected by a near-IR excess typical of Herbig AeBe stars (Lada \& Adams 1992). Klochkova $\&$ Chentsov (2004) present evidence of a line radial velocity gradient that can be interpreted as an indication of matter infall. These findings suggest the existence of an accretion disk around the star.

The XMM-Newton EPIC spectrum of Cygnus OB2 \#12 was also fitted by Rauw et al. (2005), who find temperatures $k T_{1}=0.73 \pm 0.16$ and $k T_{2}=1.8 \pm 0.4 \mathrm{keV}$, consistent with our own estimation (see Fig. 12 and Table 4). The temperature of the hard component is significantly higher than expected according to the wind shock emission model, given the low velocity of the stellar wind, $\sim 150 \mathrm{~km} \mathrm{~s}^{-1}$. Emission from MCWS might better explain the spectrum (see above). Such a scenario could also explain the observed X-ray variability (see Sect. 5) if the magnetosphere is tilted with respect to the rotation axis as in the case of $\theta^{1}$ Orionis $C$ (Gagné et al. 2005).

\subsection{Intermediate-mass stars}

Intermediate-mass stars $\left(2.8<M / M_{\odot}<10\right)$ are not expected to emit X-rays because, unlike $\mathrm{O}$ - and early B stars, they do not drive strong stellar winds nor possess outer convective zones that can sustain a dynamo mechanism such as the one that is ultimately held responsible for X-ray activity in low-mass stars. Several studies have however observed X-ray emission apparently associated with late B- and A-type stars. Although its origin has been often attributed to the coronal activity of unresolved late-type companions (e.g. Stelzer et al. 2006a,b), the matter is controversial. The Cygnus OB2 region has a large number of intermediate mass stars. It is therefore an ideal target to check this hypothesis. We classify 66 ACIS sources as intermediatemass. The $L_{X}$ vs. mass plot in Fig. 11 does not reveal any dramatic discontinuity at the boundaries with low and high mass stars. Intermediate mass stars, however, do not seem to follow the trend of increasing $L_{\mathrm{X}}$ with mass of lower mass stars, and seem to have $L_{X}$ values that span the whole range spanned by these latter. This observation is consistent with the companion scenario.

As further supporting evidence for this conclusion we note that 8 of the X-ray sources associated with intermediate mass stars (about $12 \%$ of the sample) are variable, which is almost the same fraction found for low-mass stars (see Sect. 6.3). Moreover, their X-ray spectra are well represented by isothermal models with median $k T=2.6 \mathrm{keV}$, also in agreement with values found for low mass stars (see Sect. 7.3).

At the distance of Cygnus OB2 an offset between IR and $\mathrm{X}$-ray positions of $1^{\prime \prime}$, i.e. roughly the on-axis Chandra spatial resolution, corresponds to a projected separation of 1450 AU. X-ray source positions can however be determined with a precision of $\sim 0.1^{\prime \prime}$ ( $145 \mathrm{AU}$ ) or even better, depending on the source statistics and off-angle. If the intermediate-mass primary of a binary system is X-ray dark we should thus be able to distinguish, at least statistically, whether the observed X-ray emission originates from the low mass secondary in the case of wide binary systems, defined as systems with separation $>0.001 \mathrm{pc}$ (206 AU) (cf. Close et al. 1990). Figure 13 shows an example of an X-ray source associated with an intermediate-mass counterpart, but with a significant spatial offset. A comparison of the distributions of X-ray-NIR offsets for X-ray sources associated with low and intermediate mass counterparts is however inconclusive. If the companion hypothesis is correct, most X-ray emitting low-mass companions are not in wide systems.

\subsection{Low-mass stars}

The origin of X-ray activity in low-mass PMS stars has so far eluded full understanding. Although many aspects of MS coronal activity also are not understood, the X-ray activity of MS 


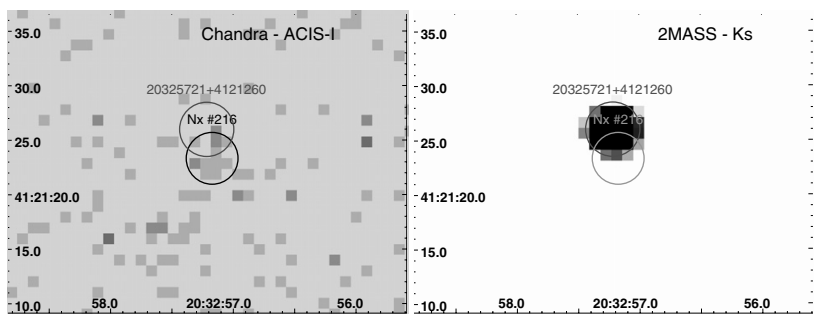

Fig. 13. Example of a possible misidentification of an X-ray source with a intermediate-mass near-IR star. We show the X-ray and $K_{\mathrm{s}}$-band images of ACIS source \#216. The X-ray and 2MASS positions differ by $\sim 2.5 \operatorname{arcsec}\left(\sim 1.8 \times 10^{-2} \mathrm{pc}\right.$ at the Cygnus OB2 distance). The X-ray source might be instead associated with a faint near-IR source undetected in 2MASS.

stars correlates well with stellar rotational and convection properties, thus supporting the picture of a solar-like corona that is ultimately powered by a stellar dynamo. For Pre-Main Sequence (PMS) stars, however, no such a correlation has been found and the picture appears somewhat complicated by the presence of accretion disks, which seem to play an important role in the $\mathrm{X}$-ray emission. On one hand plasma heated by shocks due to the accretion process is likely to be a source of soft X-rays (cf. Kastner et al. 2002; Stelzer \& Schmitt 2004; Favata et al. 2005; Flaccomio et al. 2006). On the other hand the presence of accretion disks seems to lower the overall activity level, although probably making the X-ray emission harder and more time variable (Flaccomio et al. 2003; Preibisch et al. 2005; Flaccomio et al. 2006).

We have defined the low mass range $M<2.8 M_{\odot}$, based on the presence of a significant convection envelope. The X-ray spectra and luminosities of low mass stars in Cygnus OB2 are typical of T-Tauri stars in other regions: our spectral analysis (Sect. 6) yields a median $k T=2.38 \mathrm{keV}$ and $\log N_{\mathrm{H}}=22.23$. Figure 10 indicates that, although the spread of $L_{\mathrm{x}}$ at any given mass is of the order of 1 dex, a trend of increasing $L_{x}$ with mass is observed. The same figure shows for comparison the nearly complete sample of Orion Nebular Cluster (ONC) stars as observed by the Chandra Orion Ultra-deep Project (COUP) (Getman et al. 2005b). The sensitivity limits of the two observations are quite different because of the larger distance of Cygnus OB2 ( $\sim 1450$ pc vs. 470 pc for the ONC), and the longer exposure time of the COUP observation ( $\sim 838$ vs. $\sim 97 \mathrm{ks}$ for our data). However, as already discussed, the most severe limit to the completeness of our sample of likely members with nearIR counterparts is likely to be the sensitivity limit of the 2MASS data. The Cygnus OB2 stars in Fig. 10 have a well defined limit of mass, at $\sim 0.4-0.5 M_{\odot}$, rather than X-ray luminosity.

Figure 10 shows that the luminosities of low mass stars in Cygnus OB2 and in the ONC are compatible. The numbers of stars in the 1.0-2.8 $M_{\odot}$ mass range in the Cygnus OB2 and ONC regions differ considerably. In Cygnus OB2 we count 334 such stars, about 10 times as many as the in a sample of 375 COUPdetected lightly absorbed $\left(A_{\mathrm{v}}<2\right)$ ONC stars with mass estimates, in which we find 34 stars in the same range (Getman et al. 2005b). Assuming that the IMFs in the two regions are the same and that the considered sample of lightly absorbed ONC stars is complete, we can estimate the total number of stars in our Cygnus OB2 ACIS FOV as $375 / 34 \times 334 \sim 3700$. Considering that our FOV contains only one fourth of the 106 OB stars known in Cygnus OB2, we can then scale this number by the number of OB stars to get the total expected Cygnus OB2 population: $\sim 15000$ stars. The total stellar mass of the cluster can be then estimated by multiplying this number by the average stellar mass in the lightly absorbed COUP sample $\left(0.84 M_{\odot}\right): M_{\mathrm{cl}} \sim$ $1.2 \times 10^{4} M_{\odot}$, somewhat lower than the estimate of Knödlseder (2000), who derived 4-10 $\times 10^{4} M_{\odot}$.

Deeper and more extended X-ray and near-IR surveys of Cygnus OB2 will be invaluable for the study of young stars of all masses with unprecedented statistic. Such observations has been recently obtained at the TNG observatory, and we will present such results in a forthcoming paper.

\section{Summary and conclusions}

We have reported the results of a deep Chandra X-ray observation pointed toward the $\sim 2$ Myr old star forming region Cygnus OB2. Source detection was performed using PWDdetect, a wavelet-based algorithm, supplemented by visual inspection, identifying 1003 X-ray sources in the $17^{\prime} \times 17^{\prime}$ ACIS-I field of view. Data extraction was performed using the semi-automated IDL-based ACIS EXTRACT package, which is well suited to the analysis of observations of crowded fields such as ours.

The X-ray source list was cross-identified with optical and near-IR (2MASS) catalogs: $26 \mathrm{X}$-ray sources were identified with optically characterized OB members of Cygnus OB2 (out of the 33 lying in the FOV) and 775 with 2MASS sources. Among these latter sources almost all are believed to be Cygnus OB2 members. About 80 X-ray sources without an optical/NIR counterpart are estimated to be of extragalactic nature (AGNs) while the remaining X-ray sources with no counterpart are likely associated with members that are fainter than the 2MASS completeness limit.

In order to characterize the previously unidentified likely cluster members with NIR counterparts, we placed them on NIR color-magnitude $\left(K_{\mathrm{s}}\right.$ vs. $\left.H-K_{\mathrm{s}}\right)$ and color-color $\left(H-K_{\mathrm{s}}\right.$ vs. $J-H)$ diagrams. A first estimate of interstellar extinction was obtained adopting a $2 \mathrm{Myr}$ isochrone for the low- and intermediatemass stars and assuming that $\mathrm{O}$ - and early B-type stars lie on the MS. We find a median visual absorption for OB stars of $A_{\mathrm{v}} \sim$ $5.6 \mathrm{mag}$, while low mass likely members seem to be slightly more absorbed, $A_{\mathrm{v}} \sim 7.0 \mathrm{mag}$. We also use the $2 \mathrm{Myr}$ isochrone and the $J$ magnitude to estimate masses of likely members assuming that they share the same distance and absorption. Our sample of X-ray selected members with near-IR counterparts reaches down to $M=0.4-0.5 M_{\odot}$, and is likely complete down to $\sim 1 M_{\odot}$. From the $H-K_{\mathrm{s}}$ vs. $J-H$ diagram we estimate the fraction of low-mass stars with NIR excesses (23/519), finding it to be quite low with respect to the $1 \mathrm{Myr}$ old ONC region indicating that either (i) the disk fraction decreases steeply with stellar age, or (ii) the intense photo-evaporating UV-field due to the large population of $\mathrm{O}$ stars in the region has reduced the disk lifetime.

At least 85 or 134 X-ray sources, i.e. $\sim 8.5 \%$ and $13 \%$ of the total, were found to be variable within our observation with a confidence level of $99.9 \%$ or $99 \%$, respectively. The fraction of variable sources increases with increasing source counts, likely as a result of a statistical bias. The light-curves of 97 sources indicate an impulsive, flare-like, behavior while the rest show more gradual variations of the X-ray emission. Only two of the 24 detected O- and early B-type stars are detected as variable during our $97 \mathrm{ks}$ observation, in spite of the high statistic of their light-curves. These exceptions are the B5 Ie star Cygnus\#12 (our source \#60), showing a rather linear decay of the count rate during the observation, and a B1.5 V+? star (\#979), which also shows a slow variability, possibly related to rotational modulation. In this latter case the detected emission may originate 
from the primary B1.5 component and/or from its presumably lower mass companion indicated by the spectral type published by Hanson (2003).

We modeled the ACIS X-ray spectra of sources with more than 20 photons assuming absorbed thermal emission models. Median $\log \left(N_{\mathrm{H}}\right)$ and $k T$ values of the sources are $22.25\left(\mathrm{~cm}^{-2}\right)$ and $2.36 \mathrm{keV}$, respectively. Variable sources, $P_{\mathrm{KS}}<0.01$ have harder spectra (median $k T: 3.3 \mathrm{keV}$ ) than non-variable ones (median $k T: 2.1 \mathrm{keV}$ ) and flaring sources are even harder (median $k T: 3.8$ ). Sources associated with $\mathrm{O}$ - and early B-type stars are instead quite soft (median $k T: 0.75 \mathrm{keV}$ ). Absorption corrected $\mathrm{X}$-ray luminosities of $\mathrm{OB}$ stars were calculated from the best fit spectral models. For the other, typically fainter, sources, we adopted a single count-rate to flux conversion factor computed from the results of individual spectral fits. Low mass stars have $L_{\mathrm{x}}$ ranging between $10^{30}$ and $10^{31} \mathrm{erg} \mathrm{s}^{-1}$ (median $2.5 \times 10^{30}$ ). Variable low mass stars are on average 0.4 dex brighter, probably because of a selection effect. These X-ray luminosities are consistent with those of similar mass stars in the slightly younger (1 Myr) ONC region. $\mathrm{O}$ and B-type stars are the most luminous, with $L_{\mathrm{x}}=2.5 \times 10^{30}-6.3 \times 10^{33} \mathrm{erg} \mathrm{s}^{-1}$. Their X-ray and bolometric luminosities are in rough agreement with the relation $L_{\mathrm{x}} / L_{\mathrm{bol}}=10^{-7}$, albeit with an order of magnitude dispersion. The X-ray luminosities of the 10 evolved OB stars in our sample however, seem to obey a better defined and steeper power-law relation with $L_{\mathrm{bol}}: L_{\mathrm{x}} \propto L_{\mathrm{bol}}^{1.8}$ ). Further studies of a larger number of evolved OB-type stars are needed to confirm or disprove this dependence.

Acknowledgements. This publication makes use of data products from the Two Micron All Sky Survey, which is a joint project of the University of Massachusetts and the Infrared Processing and Analysis Center/California Institute of Technology, funded by the National Aeronautics and Space Administration and the National Science Foundation. J.F.A.C acknowledges support by the Marie Curie Fellowship Contract No. MTKD-CT-2004-002769 of the project "The Influence of Stellar High Energy Radiation on Planetary Atmospheres", and the host institution INAF - Osservatorio Astronomico di Palermo (OAPA). E.F., G. M., F.D. and S.S. acknowledge financial support from the Ministero dell'Universita' e della Ricerca research grants, and ASI/INAF Contract I/023/05/0.

\section{References}

Adams, F. C., Hollenbach, D., Laughlin, G., \& Gorti, U. 2004, ApJ, 611, 360 Alexander, D. M., Bauer, F. E., Brandt, W. N., et al. 2003, AJ, 126, 539 Anders, E., \& Grevesse, N. 1989, Geochim. Cosmochim. Acta, 53, 197 Arnaud, K. 2004, AAS/High Energy Astrophysics Division, 8 Bally, J., O’Dell, C. R., \& McCaughrean, M. J. 2000, AJ, 119, 2919

Barger, A. J., Cowie, L. L., Capak, P., et al. 2003, AJ, 126, 632

Berghoefer, T. W., Schmitt, J. H. M. M., Danner, R., \& Cassinelli, J. P. 1997, A\&A, 322, 167

Bessell, M. S., \& Brett, J. M. 1989, LNP Vol. 341, Infrared Extinction and Standardization, 341, 61

Bevington, P. R. 1969, Data reduction and error analysis for the physical sciences (New York: McGraw-Hill), 1969

Bieging, J. H., Abbott, D. C., \& Churchwell, E. B. 1989, ApJ, 340, 518

Broos, P., Townsley, L., Getman, K., \& Bauer, F. 2002, ACIS Extract, An ACIS Point Source Extraction Package,

http://www. astro.psu.edu/xray/docs/TARA/

Chen, W., \& White, R. L. 1991, ApJ, 366, 512

Close, L. M., Richer, H. B., \& Crabtree, D. R. 1990, AJ, 100, 1968

Damiani, F., Maggio, A., Micela, G., \& Sciortino, S. 1997a, ApJ, 483, 350

Damiani, F., Maggio, A., Micela, G., \& Sciortino, S. 1997b, ApJ, 483, 370

Damiani, F., Flaccomio, E., Micela, G., et al. 2003, ApJ, 588, 1009

De Becker, M., \& Rauw, G. 2005, in Massive Stars and High-Energy Emission in OB Associations, ed. G. Rauw, Y. Nazé, \& E. R. Blomme, Gosset, 73
Favata, F., \& Micela, G. 2003, Space Sci. Rev., 108, 577

Favata, F., Flaccomio, E., Reale, F., et al. 2005, ApJS, 160, 469

Feigelson, E. D., Broos, P., Gaffney, J. A., et al. 2002, ApJ, 574, 258

Feldmeier, A., Puls, J., \& Pauldrach, A. W. A. 1997, A\&A, 322, 878

Flaccomio, E., Damiani, F., Micela, G., et al. 2003, ApJ, 582, 382

Flaccomio, E., Micela, G., Sciortino, S., et al. 2005, ApJS, 160, 450

Flaccomio, E., Micela, G., \& Sciortino, S. 2006, ArXiv Astrophysics e-prints

Gagné, M., Oksala, M. E., Cohen, D. H., et al. 2005, ApJ, 634, 712

Garmire, G. P., Bautz, M. W., Ford, P. G., Nousek, J. A., \& Ricker, G. R. 2003, in X-Ray and Gamma-Ray Telescopes and Instruments for Astronomy, ed. Joachim E. Truemper, \& Harvey D. Tananbaum, Proc. SPIE, 4851, 28

Getman, K. V., Feigelson, E. D., Townsley, L., et al. 2002, ApJ, 575, 354 Getman, K. V., Feigelson, E. D., Grosso, N., et al. 2005a, ApJS, 160, 353 Getman, K. V., Flaccomio, E., Broos, P. S., et al. 2005b, ApJS, 160, 319 Guarcello, M., Prisinzano, L., Micela, G., et al. 2006, A\&A, in prep. Hanson, M. M. 2003, ApJ, 597, 957

Harnden, F. R., Branduardi, G., Gorenstein, P., et al. 1979, ApJ, 234, L51

Herrero, A., Puls, J., \& Najarro, F. 2002, A\&A, 396, 949

Hillenbrand, L. A. 2005, ArXiv Astrophysics e-prints

Johnson, H. L., \& Morgan, W. W. 1954, ApJ, 119, 344

Kastner, J. H., Huenemoerder, D. P., Schulz, N. S., Canizares, C. R., \& Weintraub, D. A. 2002, ApJ, 567, 434

Kenyon, S. J., \& Hartmann, L. 1995, ApJS, 101, 117

Kitamoto, S., \& Mukai, K. 1996, PASJ, 48, 813

Klochkova, V. G., \& Chentsov, E. L. 2004, Astron. Rep., 48, 1005

Knödlseder, J. 2000, A\&A, 360, 539

Knödlseder, J., Cerviño, M., Le Duigou, J.-M., et al. 2002, A\&A, 390, 945

Lada, C. J., \& Adams, F. C. 1992, ApJ, 393, 278

Lucy, L. B., \& White, R. L. 1980, ApJ, 241, 300

Martins, F., Schaerer, D., \& Hillier, D. J. 2005, A\&A, 436, 1049

Massey, P., \& Thompson, A. B. 1991, AJ, 101, 1408

Meyer, M. R., Calvet, N., \& Hillenbrand, L. A. 1997, AJ, 114, 288

Morrison, R., \& McCammon, D. 1983, ApJ, 270, 119

Owocki, S. P., \& Cohen, D. H. 1999, ApJ, 520, 833

Owocki, S. P., Castor, J. I., \& Rybicki, G. B. 1988, ApJ, 335, 914

Owocki, S., Townsend, R., \& Ud-Doula, A. 2005, in Magnetic Fields in the Universe: From Laboratory and Stars to Primordial Structures, ed. E. M. de Gouveia dal Pino, G. Lugones, \& A. Lazarian, AIP Conf. Proc., 784, 239

Preibisch, T. 2003, A\&A, 401, 543

Preibisch, T., Kim, Y.-C., Favata, F., et al. 2005, ApJS, 160, 401

Press, W. H., Teukolsky, S. A., Vetterling, W. T., \& Flannery, B. P. 1992, Numerical recipes in FORTRAN. The art of scientific computing (Cambridge: University Press), 2nd ed.

Prestwich, A. H., Irwin, J. A., Kilgard, R. E., et al. 2003, ApJ, 595, 719

Raassen, A. J. J., van der Hucht, K. A., Mewe, R., et al. 2003, Adv. Space Res., 32,1161

Rauw, G., De Becker, M., \& Linder, N. 2005, in Massive Stars and High-Energy Emission in OB Associations, ed. G. Rauw, Y. Nazé, \& E. R. Blomme, Gosset, 103

Raymond, J. C., \& Smith, B. W. 1977, ApJS, 35, 419

Ryter, C. E. 1996, Ap\&SS, 236, 285

Schulte, D. H. 1958, ApJ, 128, 41

Schulz, N. S., Hasinger, G., \& Truemper, J. 1989, A\&A, 225, 48

Schulz, N. S., Canizares, C., Huenemoerder, D., \& Tibbets, K. 2003, ApJ, 595, 365

Siess, L., Dufour, E., \& Forestini, M. 2000, A\&A, 358, 593

Smith, R. K., Brickhouse, N. S., Liedahl, D. A., \& Raymond, J. C. 2001, ApJ, 556, L91

Stelzer, B., \& Schmitt, J. H. M. M. 2004, A\&A, 418, 687

Stelzer, B., Huélamo, N., Micela, G., \& Hubrig, S. 2006a, A\&A, 452, 1001

Stelzer, B., Micela, G., Hamaguchi, K., \& Schmitt, J. H. M. M. 2006b, ArXiv Astrophysics e-prints

Stevens, I. R., Blondin, J. M., \& Pollock, A. M. T. 1992, ApJ, 386, 265

Torres-Dodgen, A. V., Carroll, M., \& Tapia, M. 1991, MNRAS, 249, 1 ud-Doula, A., \& Owocki, S. P. 2002, ApJ, 576, 413

Van Loo, S. 2005, Ph.D. Thesis

Waldron, W. L., Corcoran, M. F., Drake, S. A., \& Smale, A. P. 1998, ApJS, 118, 217

Waldron, W. L., Cassinelli, J. P., Miller, N. A., MacFarlane, J. J., \& Reiter, J. C. 2004, ApJ, 616, 542

Weisskopf, M. C., Brinkman, B., Canizares, C., et al. 2002, PASP, 114, 1

Wolk, S. J., Harnden, F. R., Flaccomio, E., et al. 2005, ApJS, 160, 423 


\section{Online Material}


空品

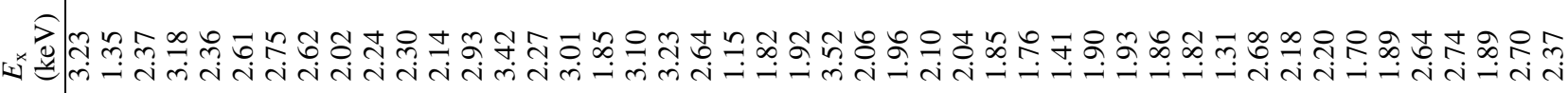

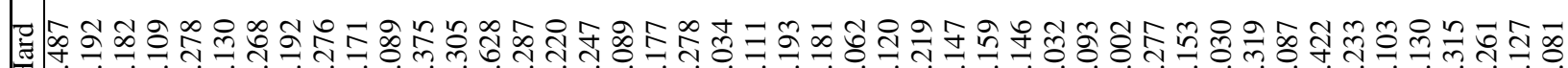

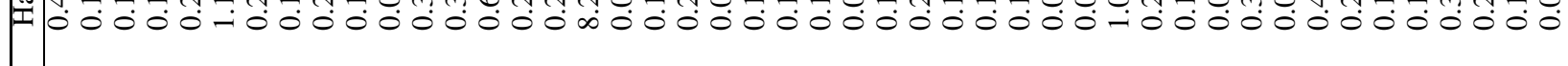
फ ज

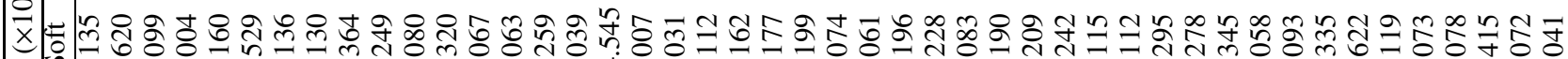
级

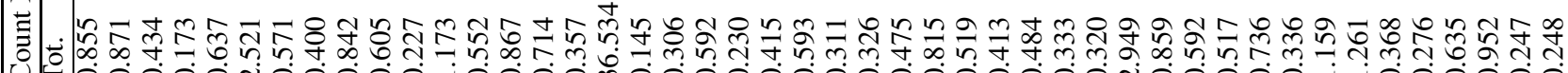
Uे

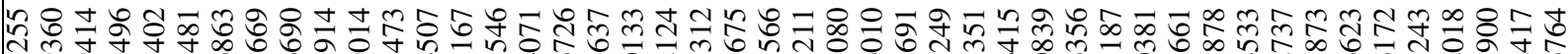

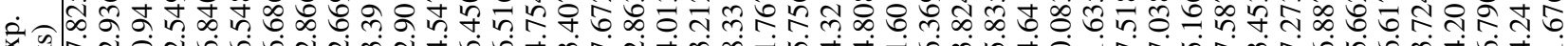

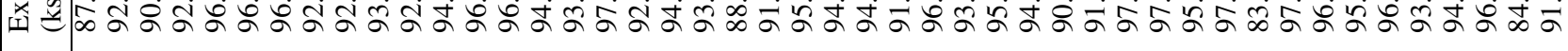

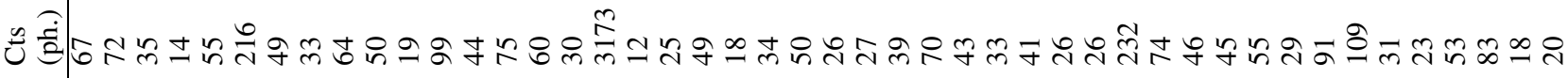
W

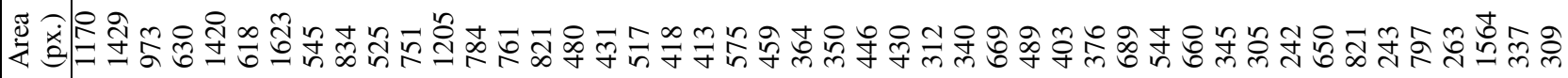

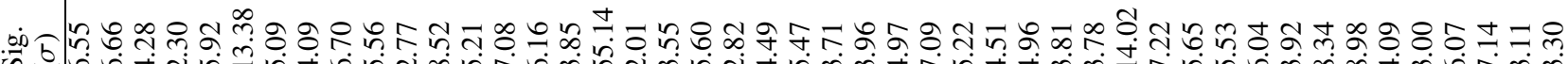

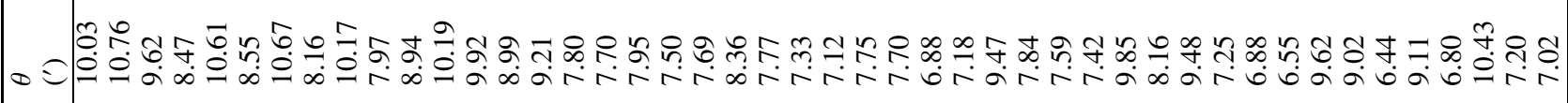

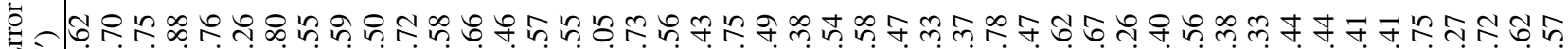

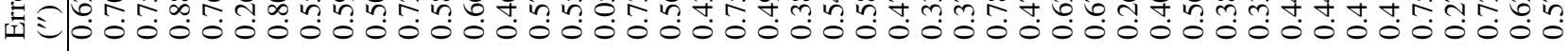

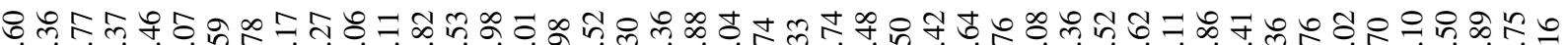
ने 苍

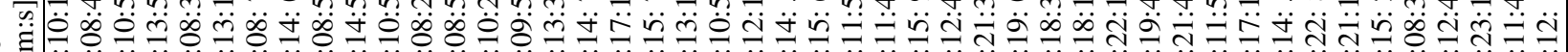

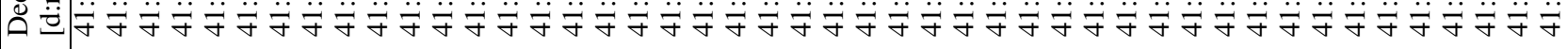

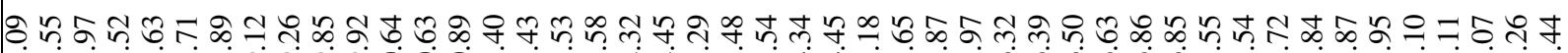

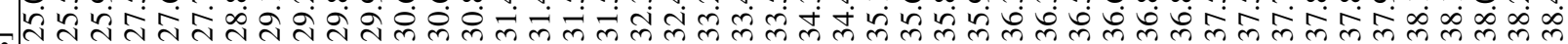

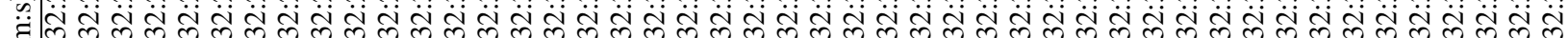

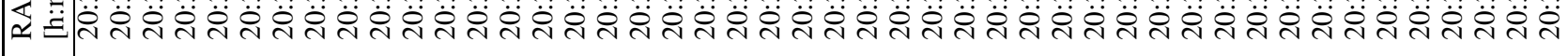

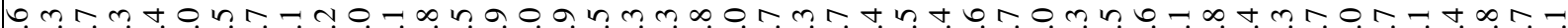

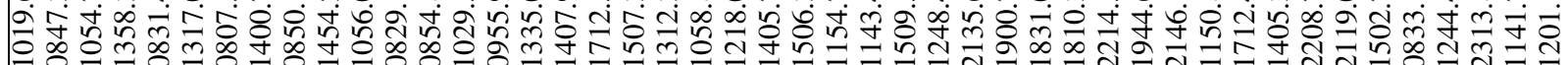

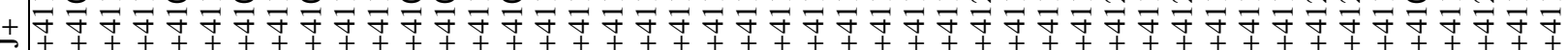
0

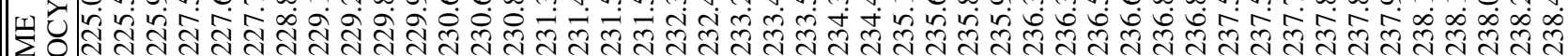

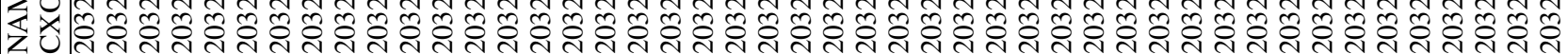

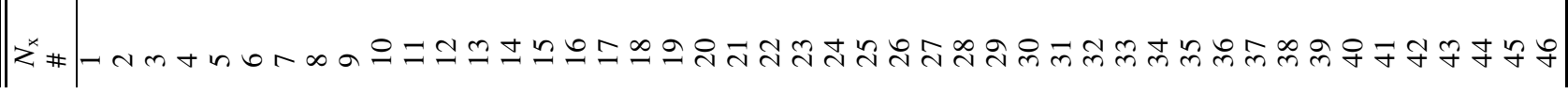


J. F. Albacete Colombo et al.: Unveiling the Cygnus OB2 stellar population with Chandra, Online Material p 3

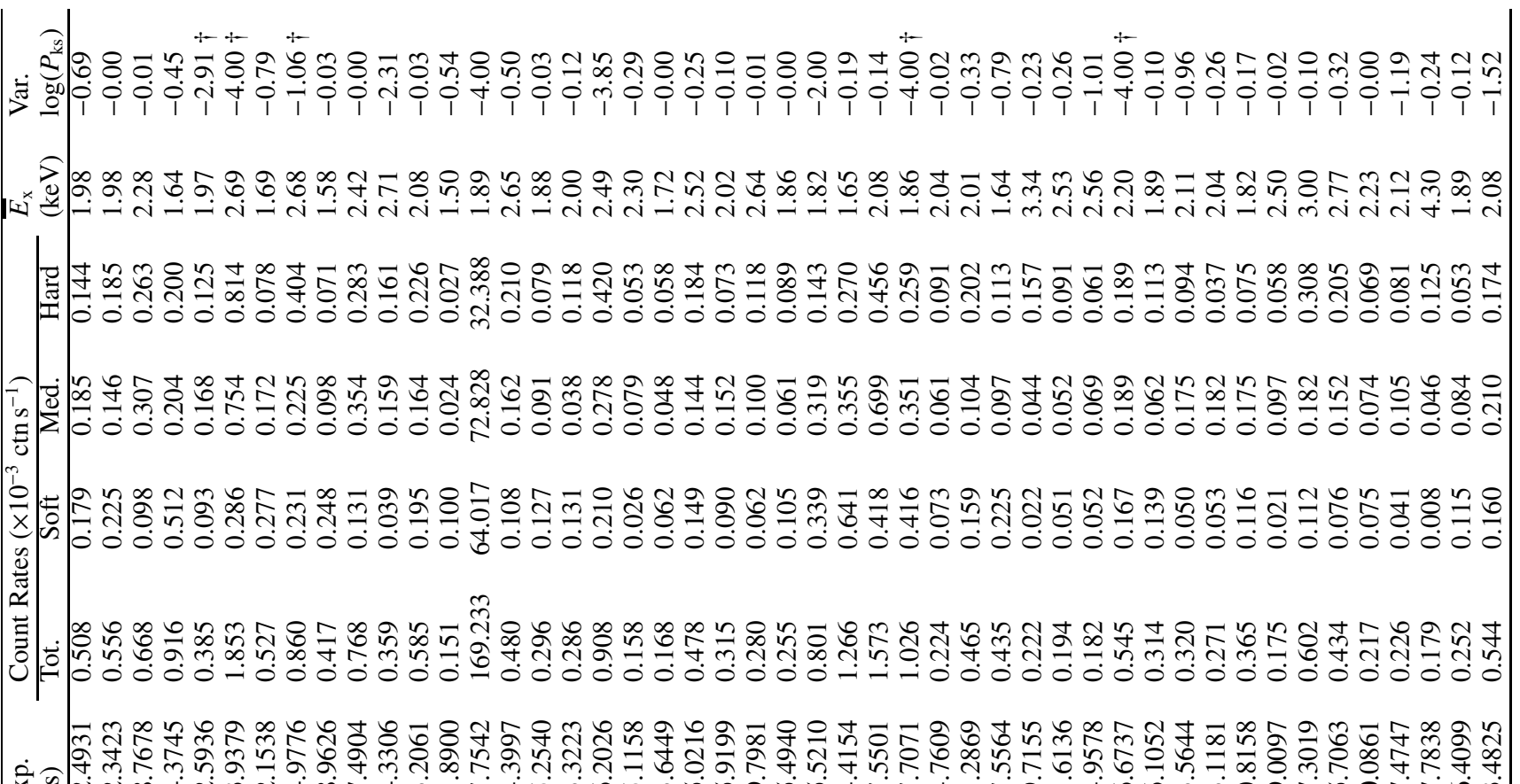

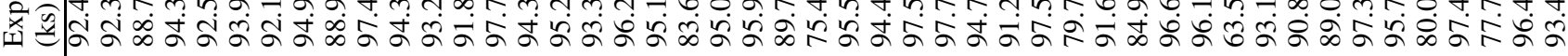

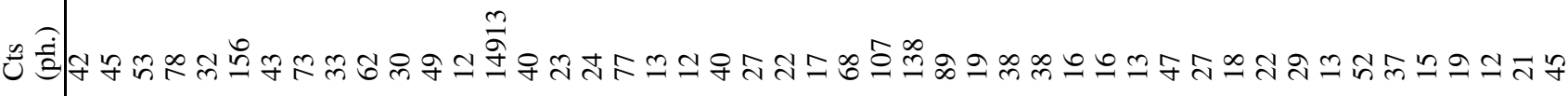

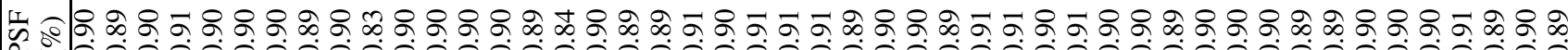
L 000000000000000000000000000000000000000000000000 娄

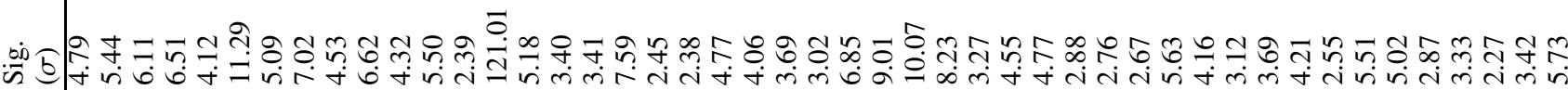

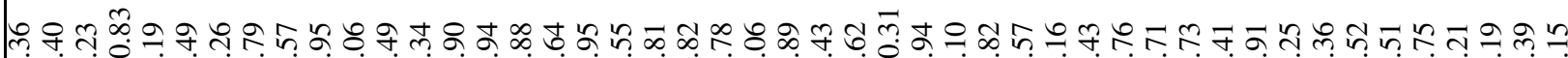

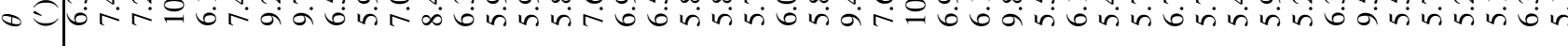

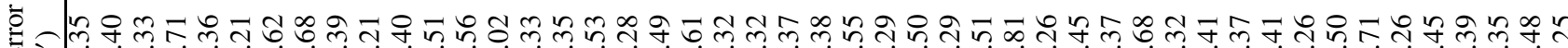

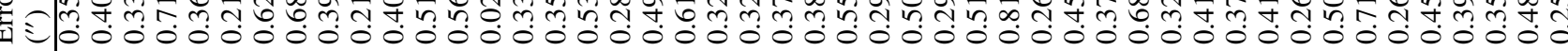

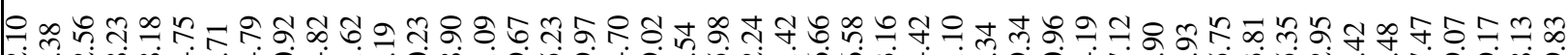
तु

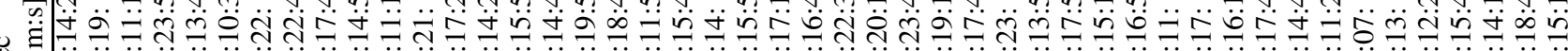

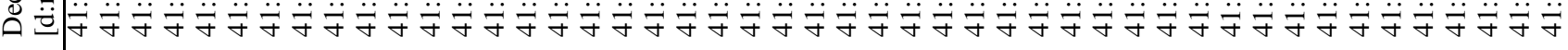

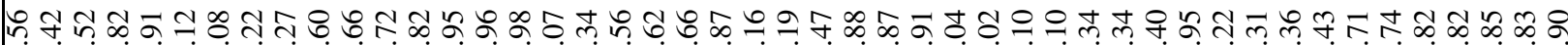

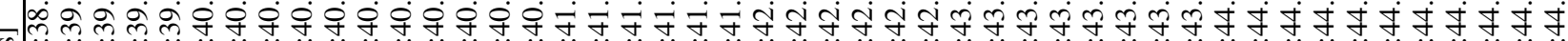

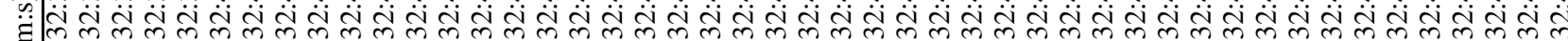

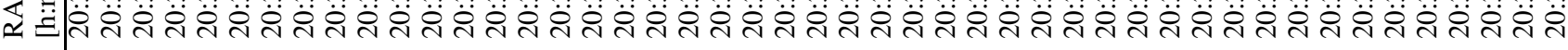

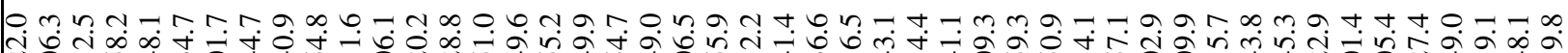

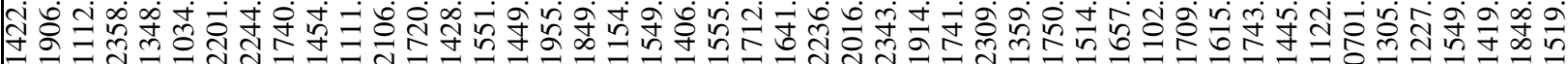

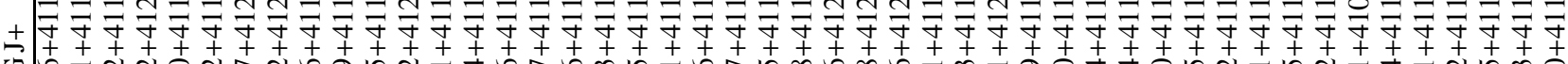

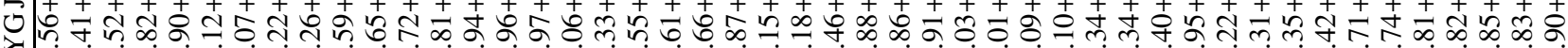
至

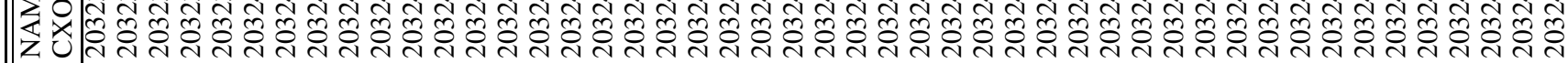


J. F. Albacete Colombo et al.: Unveiling the Cygnus OB2 stellar population with Chandra, Online Material p 4

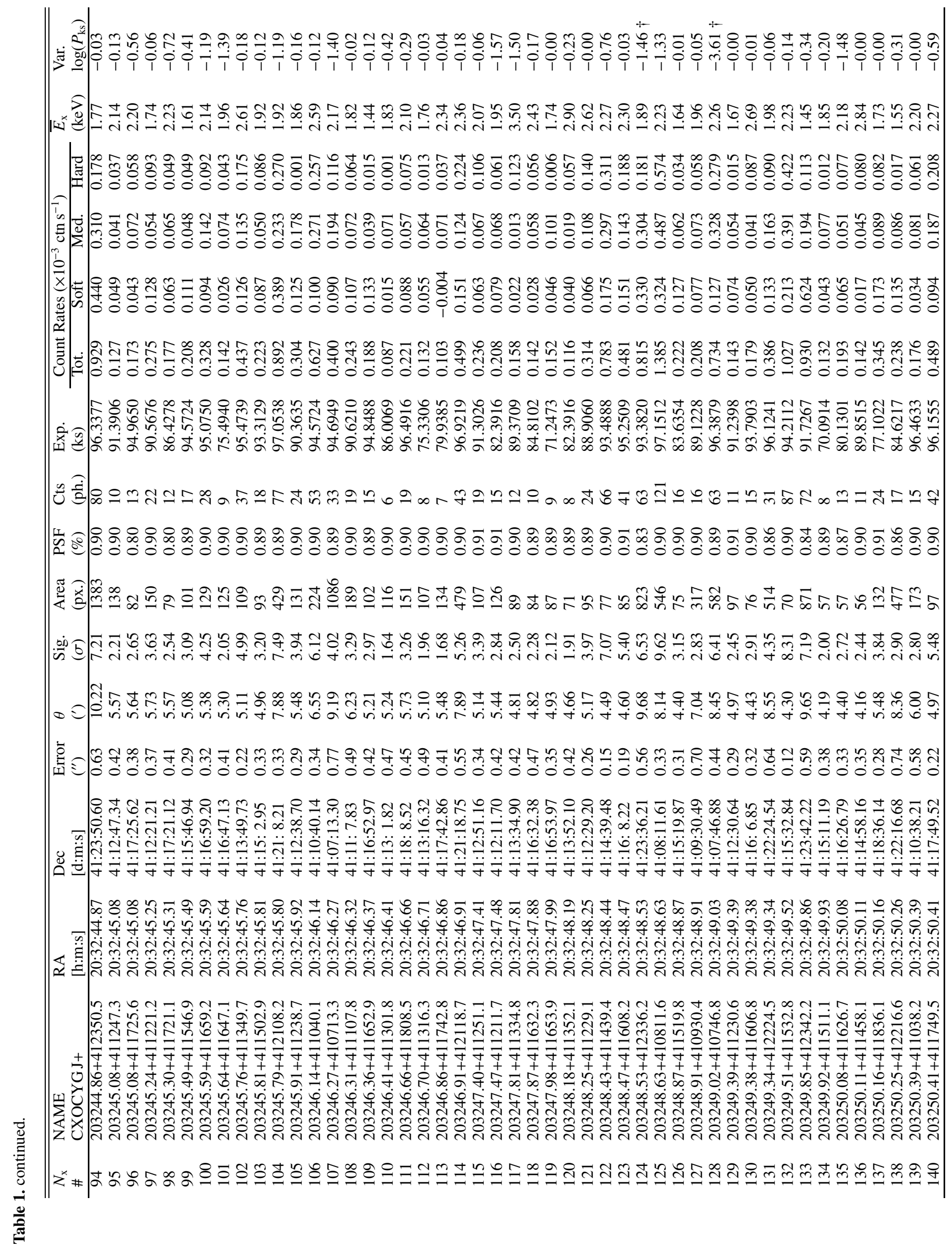


J. F. Albacete Colombo et al.: Unveiling the Cygnus OB2 stellar population with Chandra, Online Material p 5

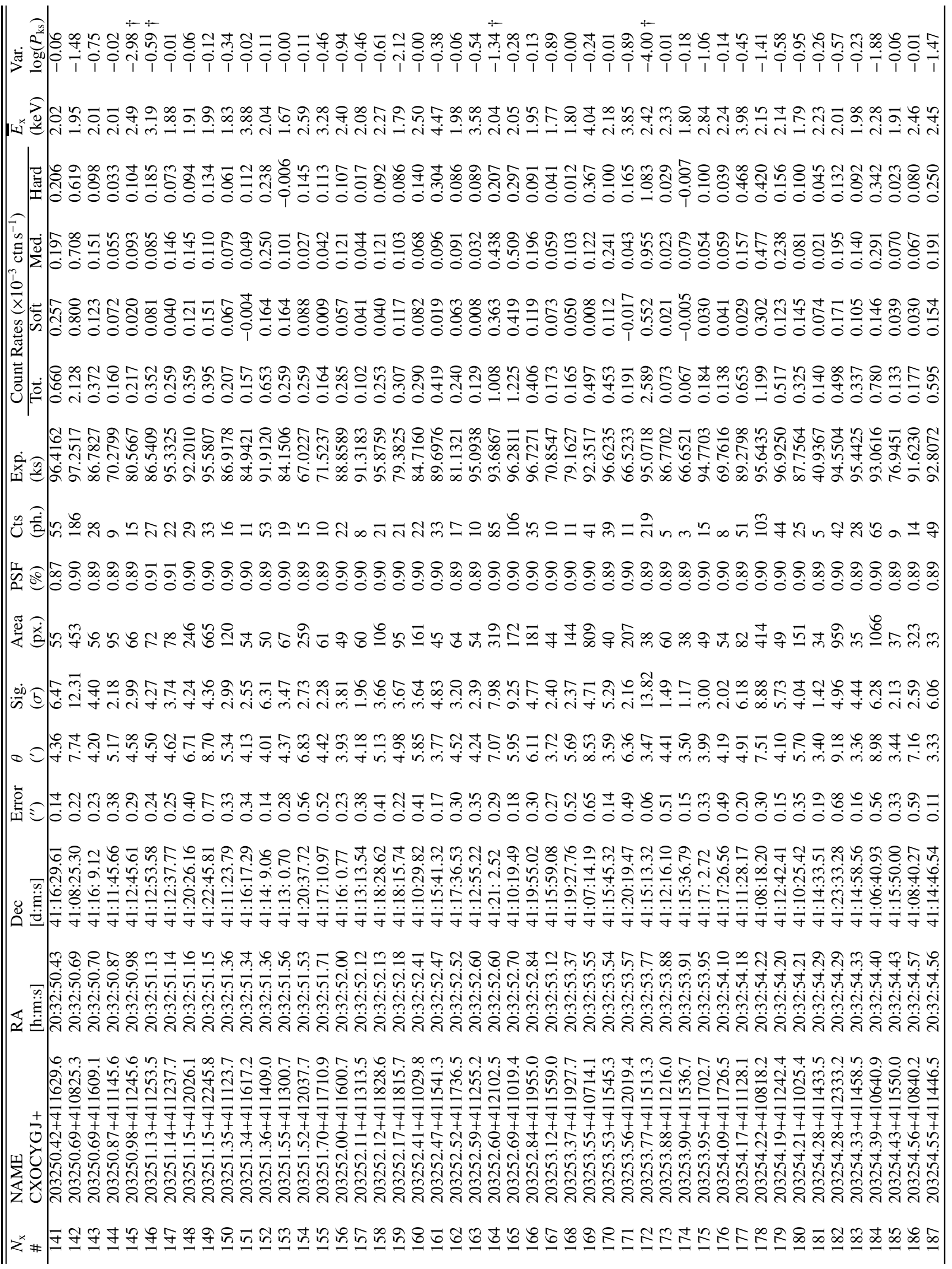




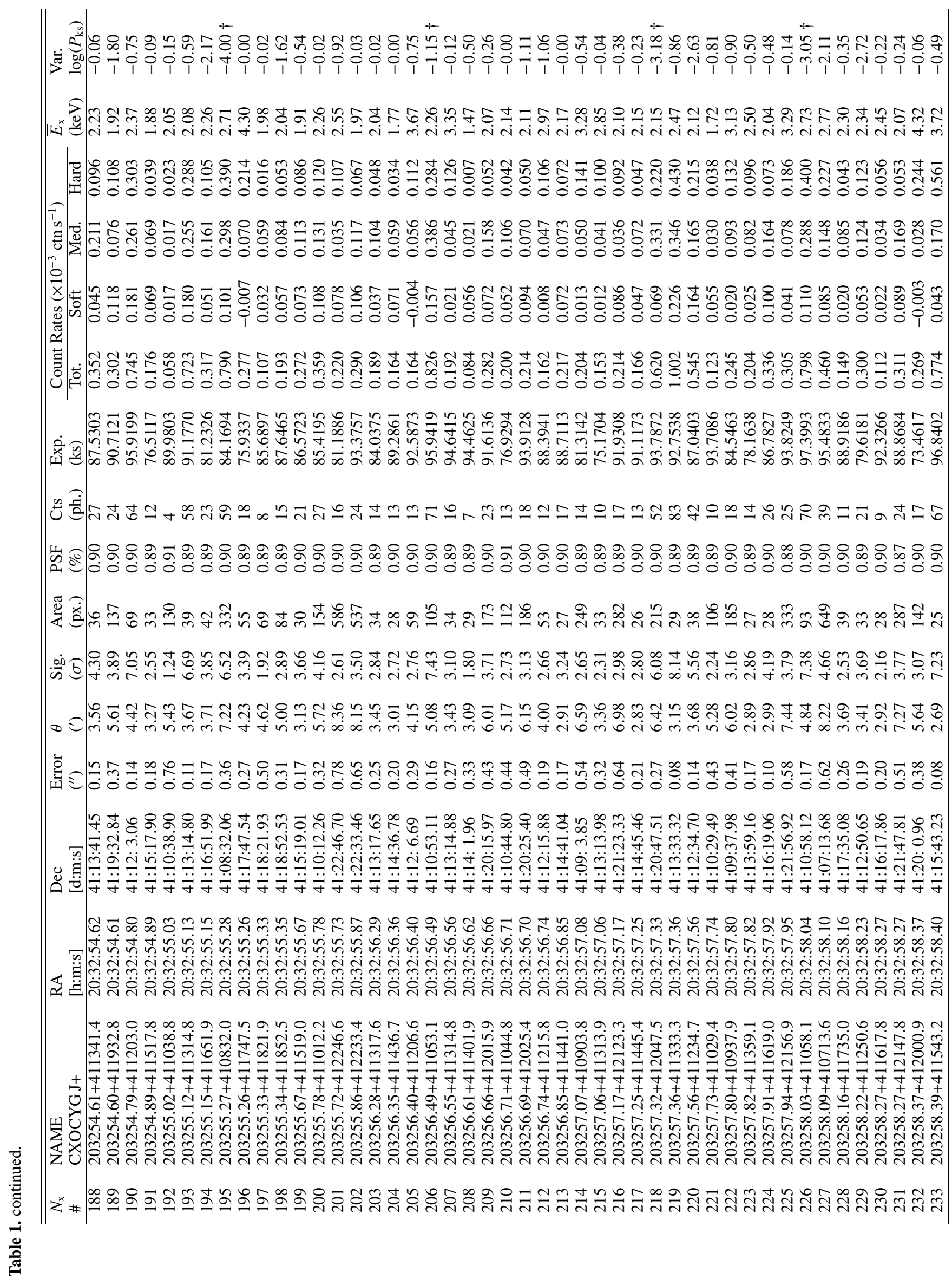




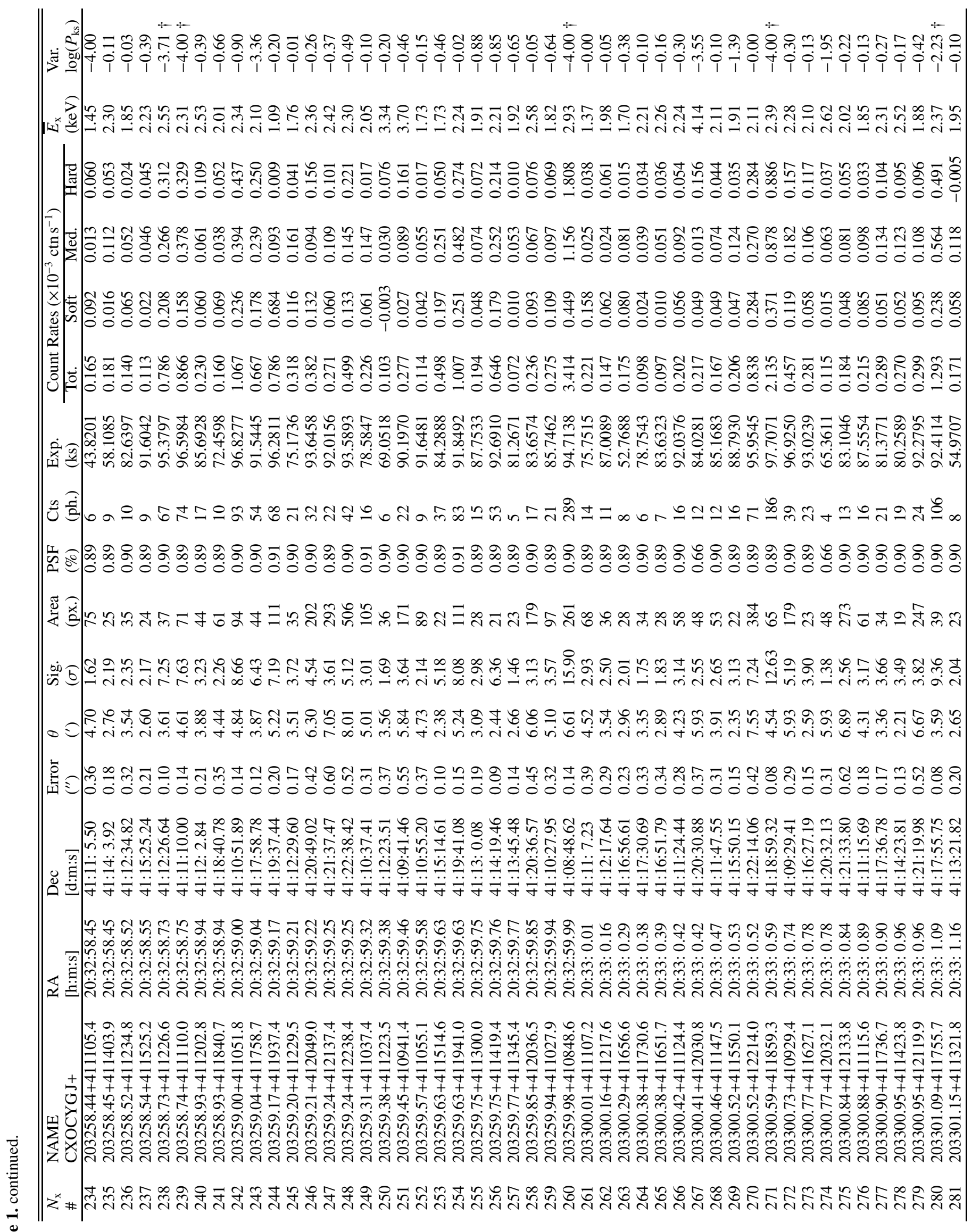


J. F. Albacete Colombo et al.: Unveiling the Cygnus OB2 stellar population with Chandra, Online Material p 8

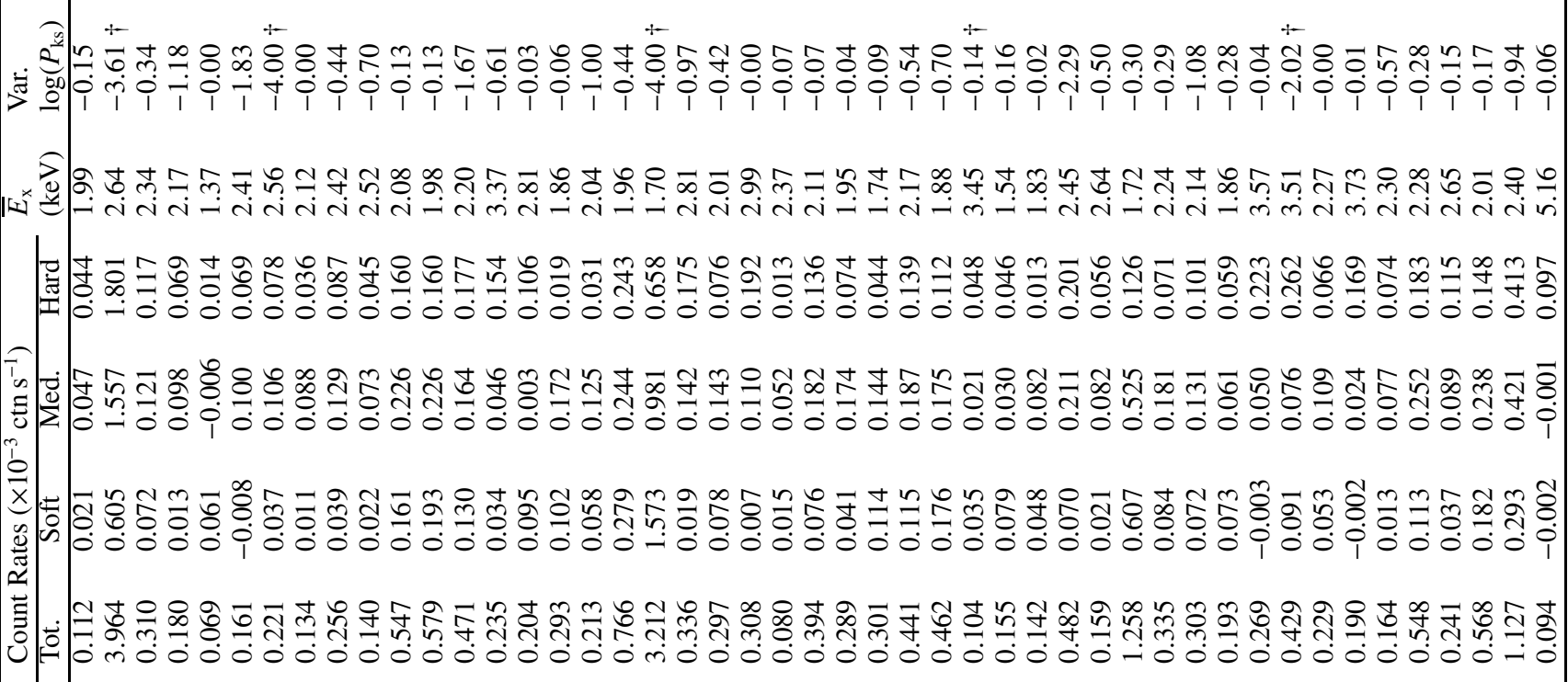

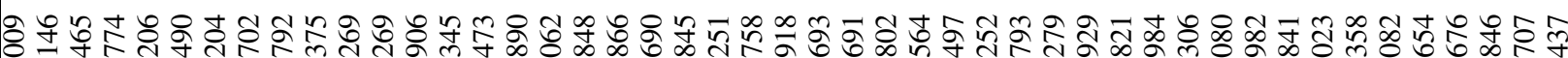

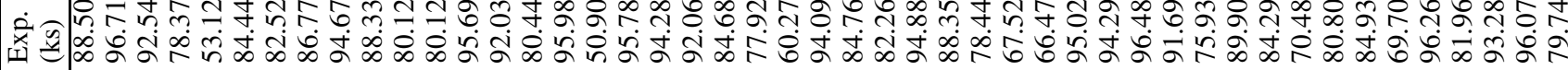

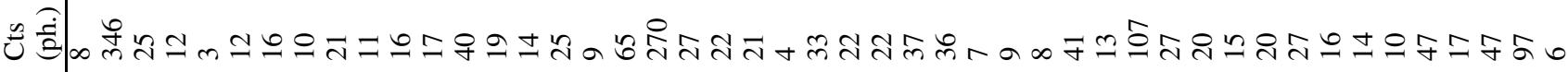

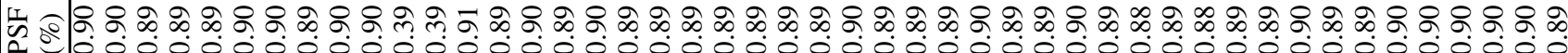
J $\widehat{\dot{J}}$

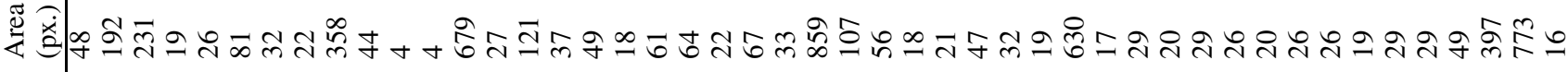

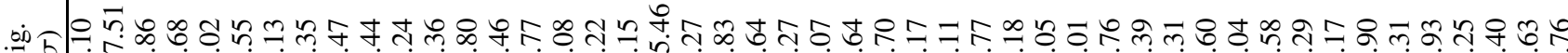
ڤ్

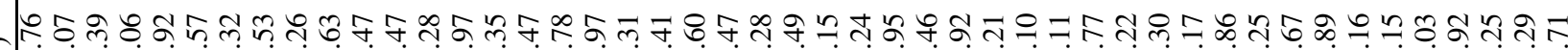

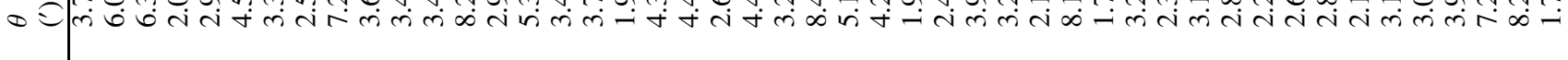

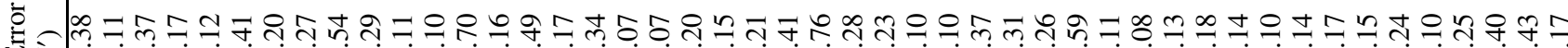

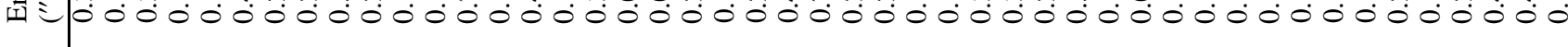

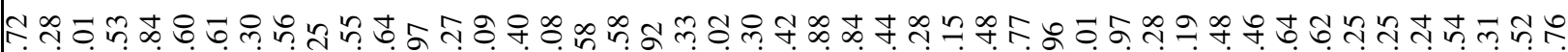

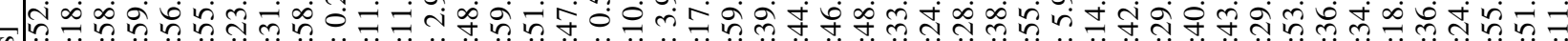

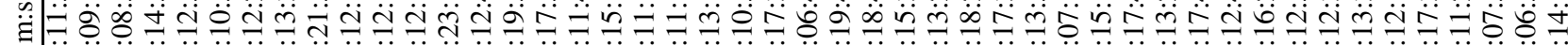

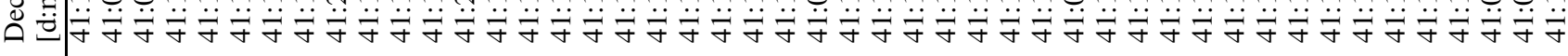

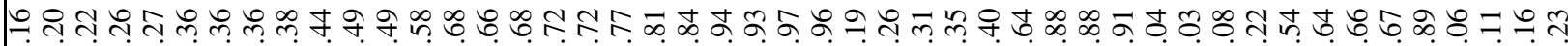

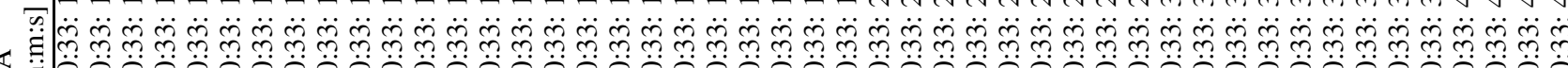

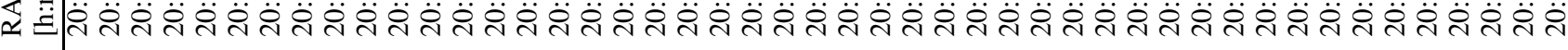

F no n $\infty$ n तिं

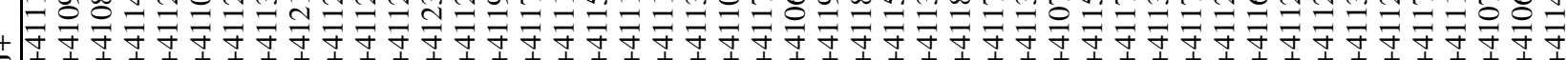

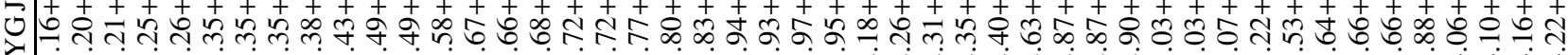

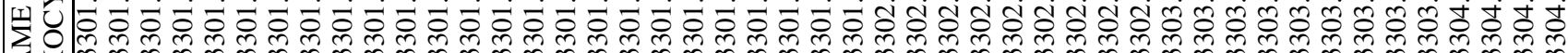

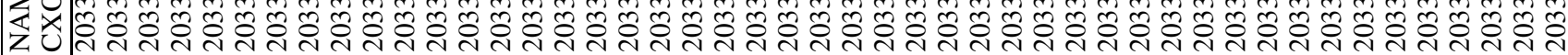

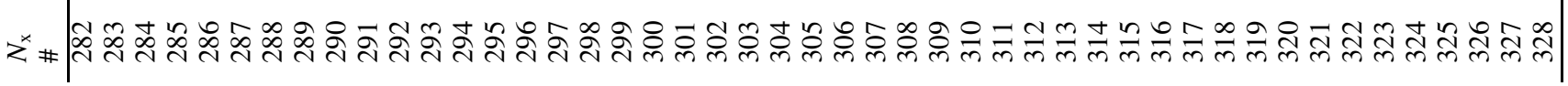


J. F. Albacete Colombo et al.: Unveiling the Cygnus OB2 stellar population with Chandra, Online Material p 9

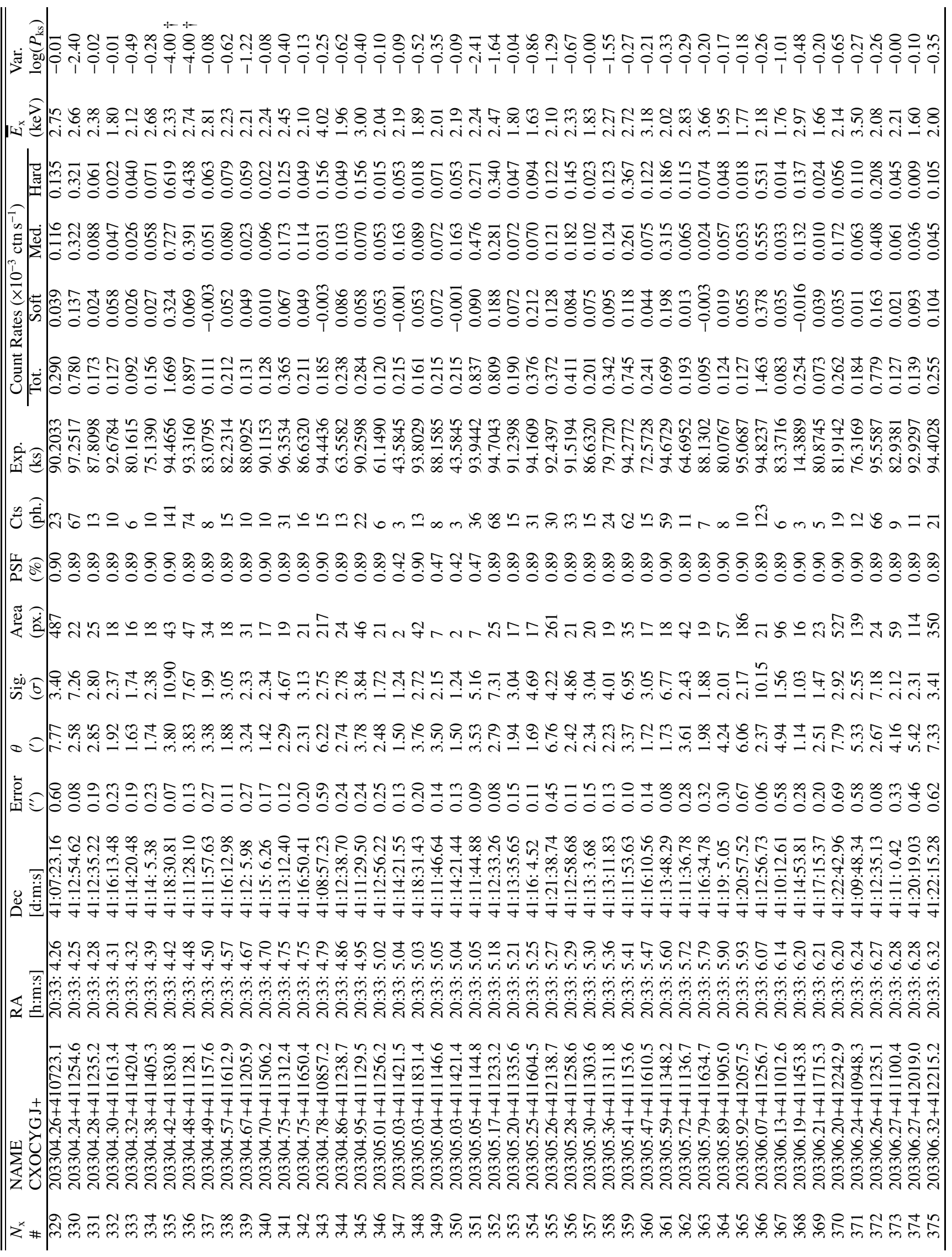




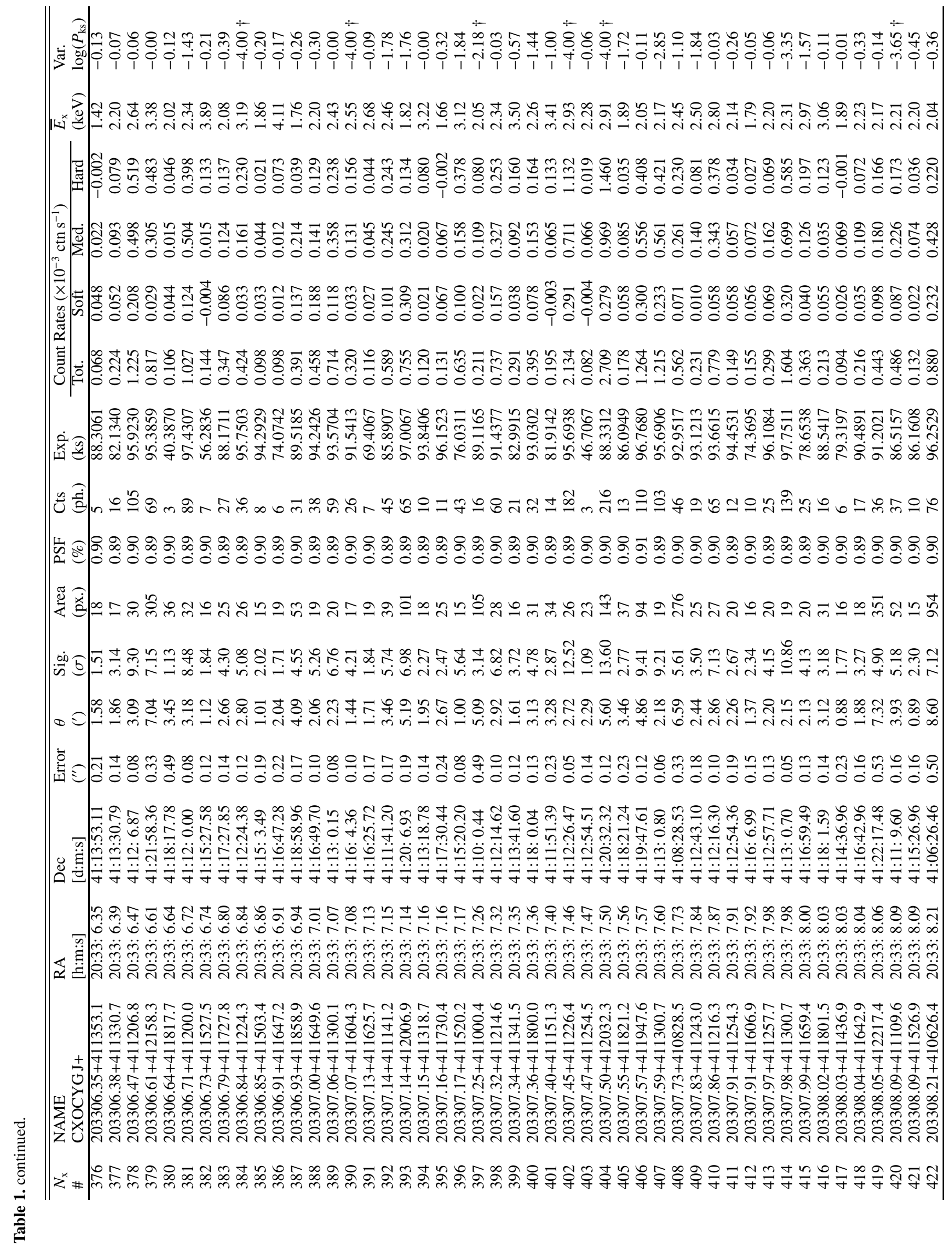


J. F. Albacete Colombo et al.: Unveiling the Cygnus OB2 stellar population with Chandra, Online Material p 11

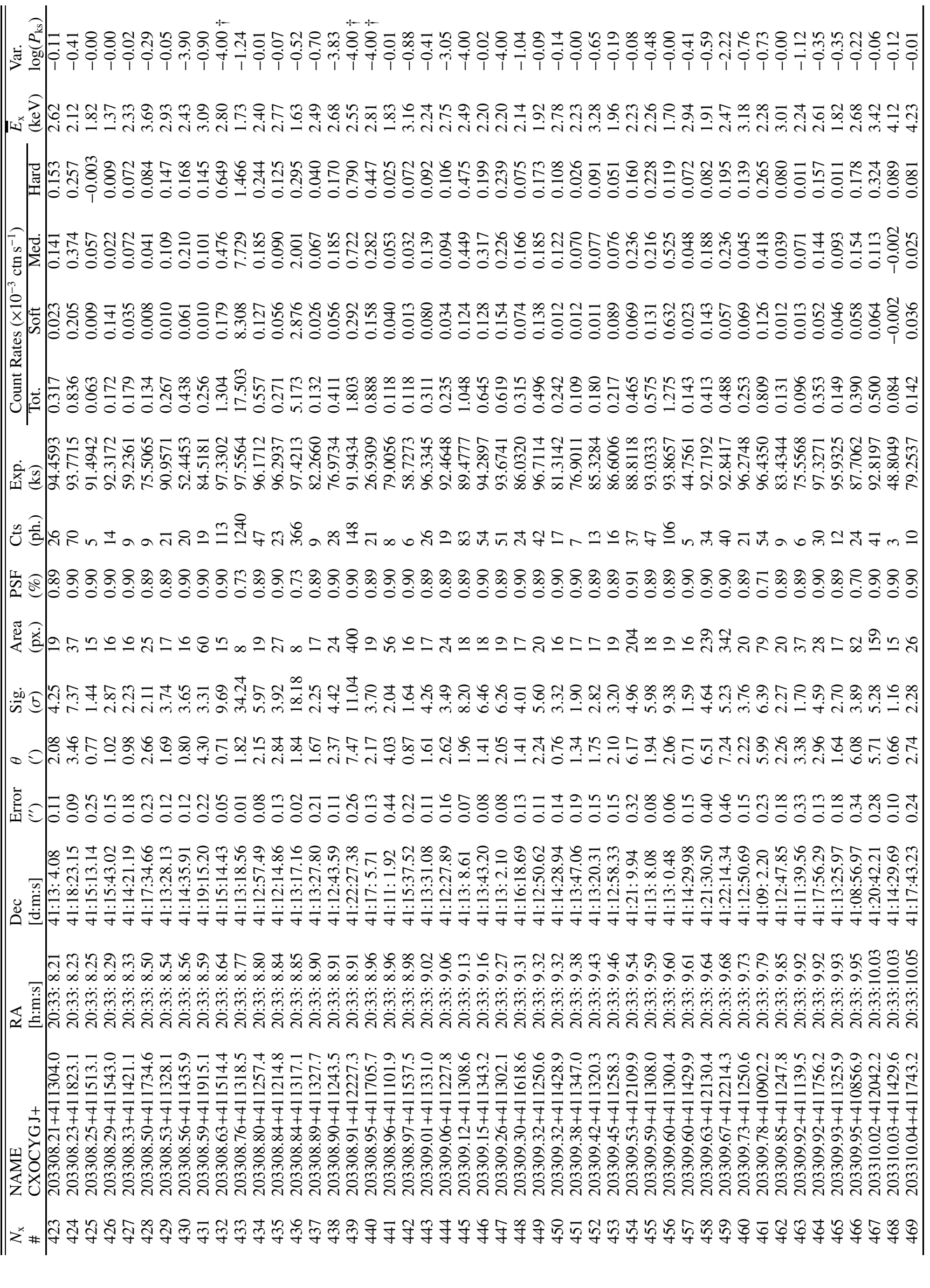


J. F. Albacete Colombo et al.: Unveiling the Cygnus OB2 stellar population with Chandra, Online Material p 12

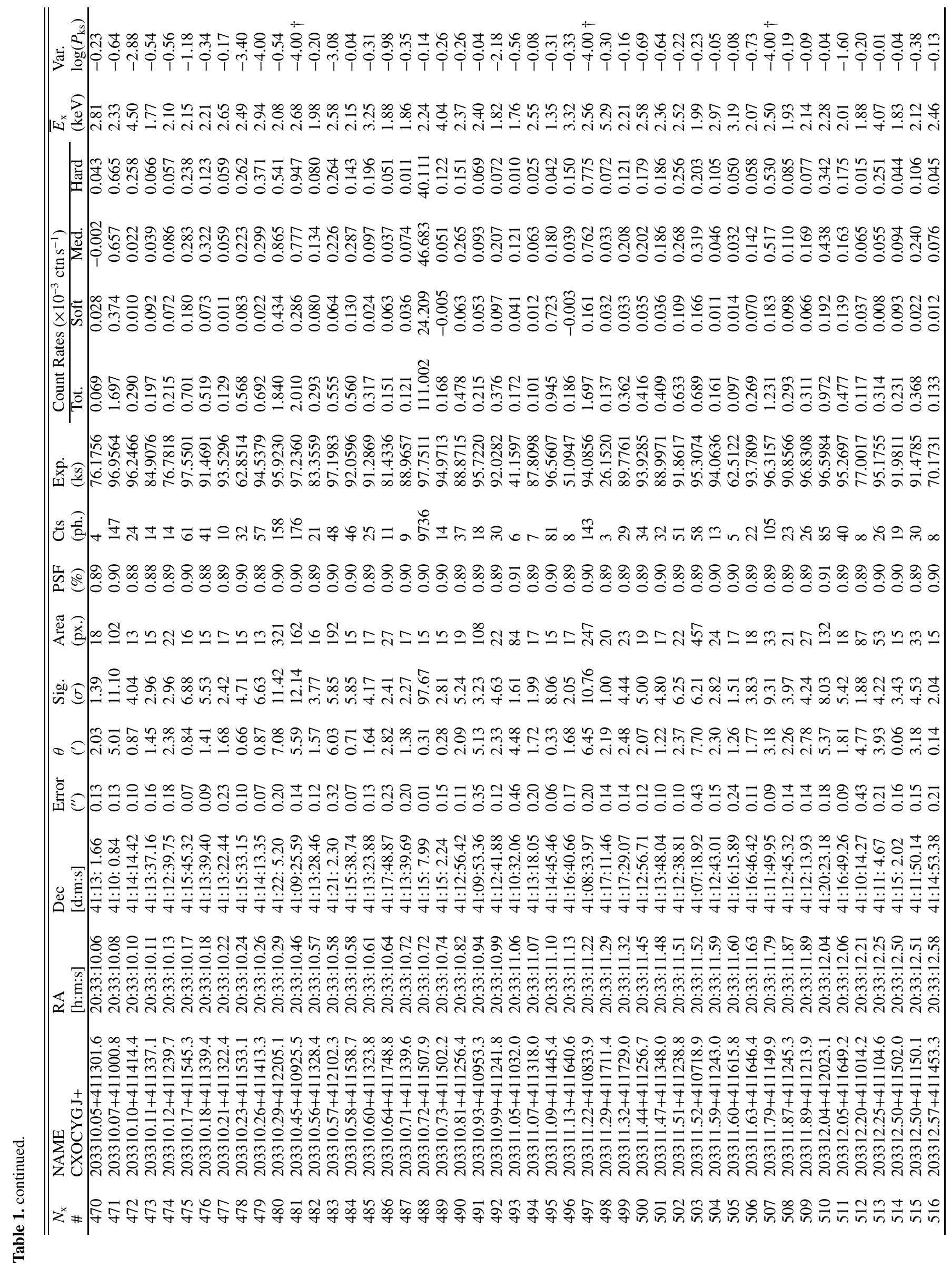


J. F. Albacete Colombo et al.: Unveiling the Cygnus OB2 stellar population with Chandra, Online Material p 13

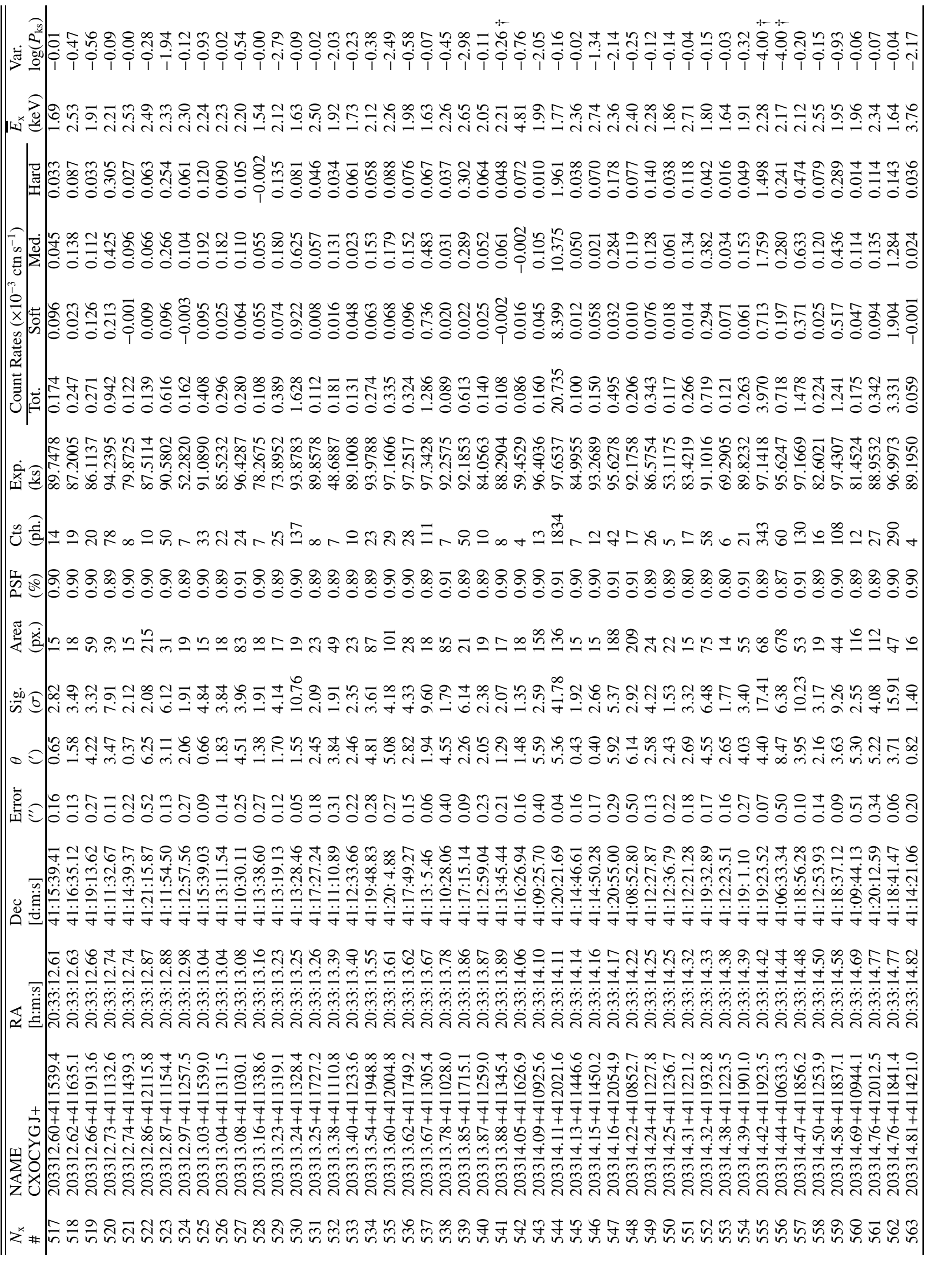


J. F. Albacete Colombo et al.: Unveiling the Cygnus OB2 stellar population with Chandra, Online Material p 14

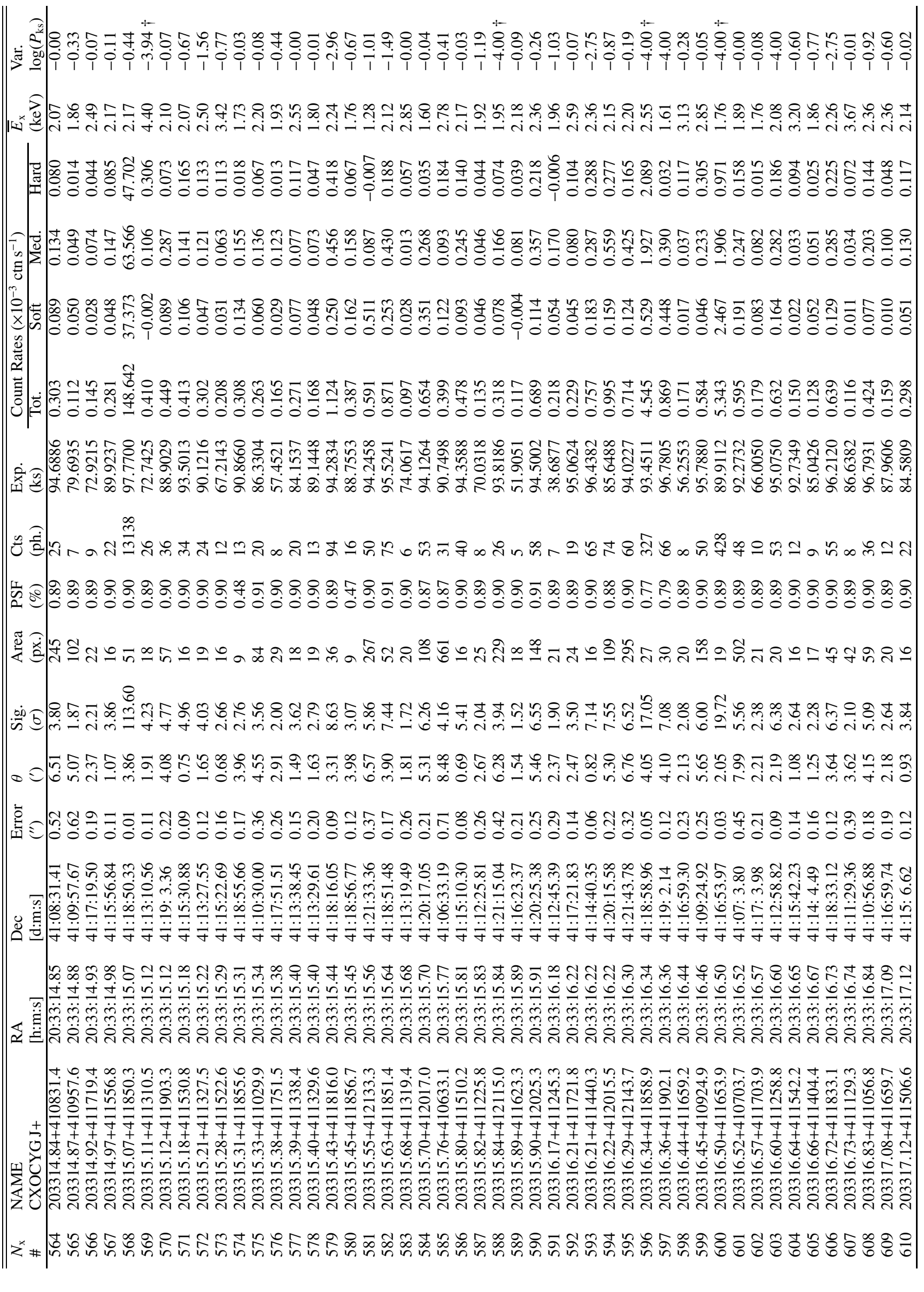


J. F. Albacete Colombo et al.: Unveiling the Cygnus OB2 stellar population with Chandra, Online Material p 15

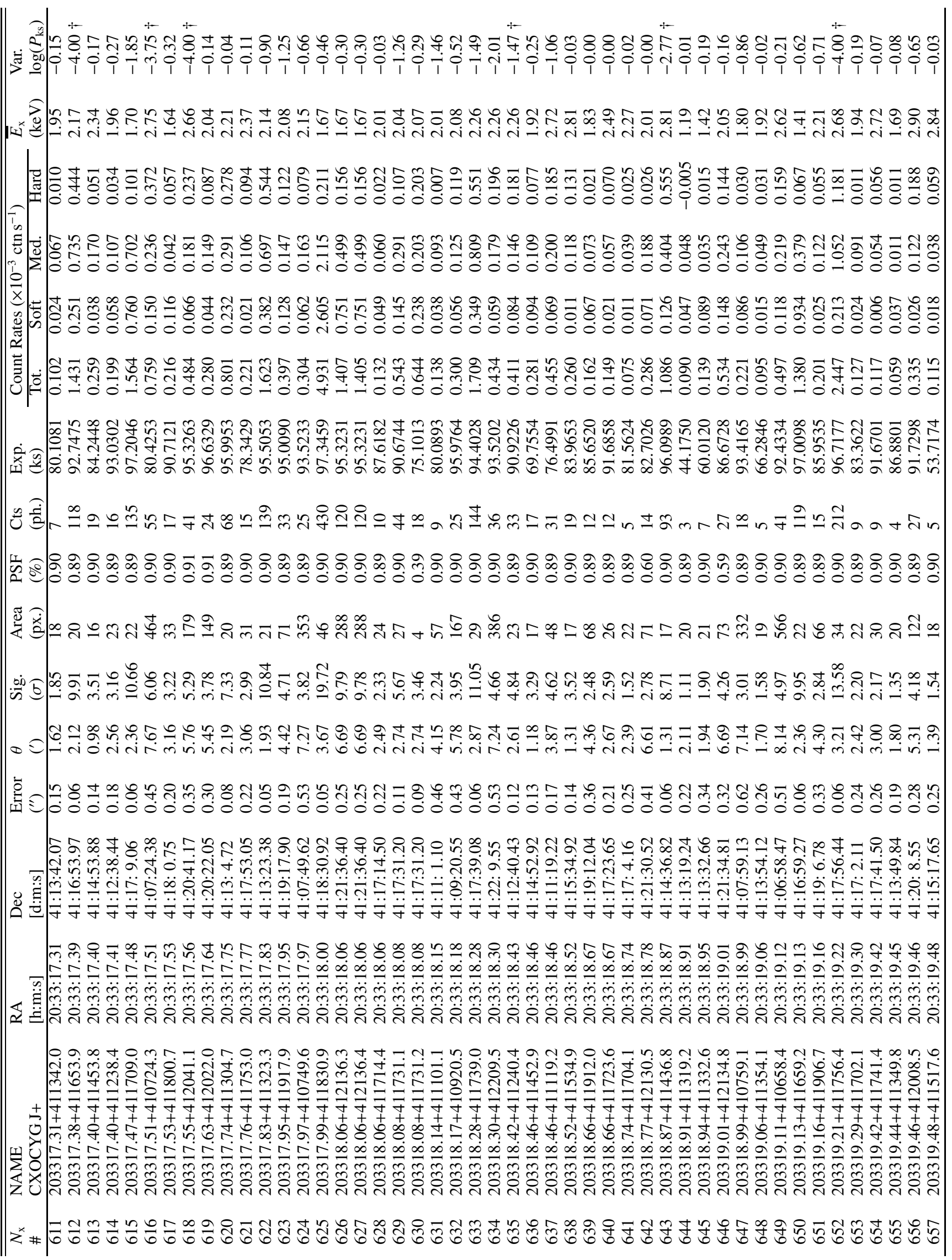




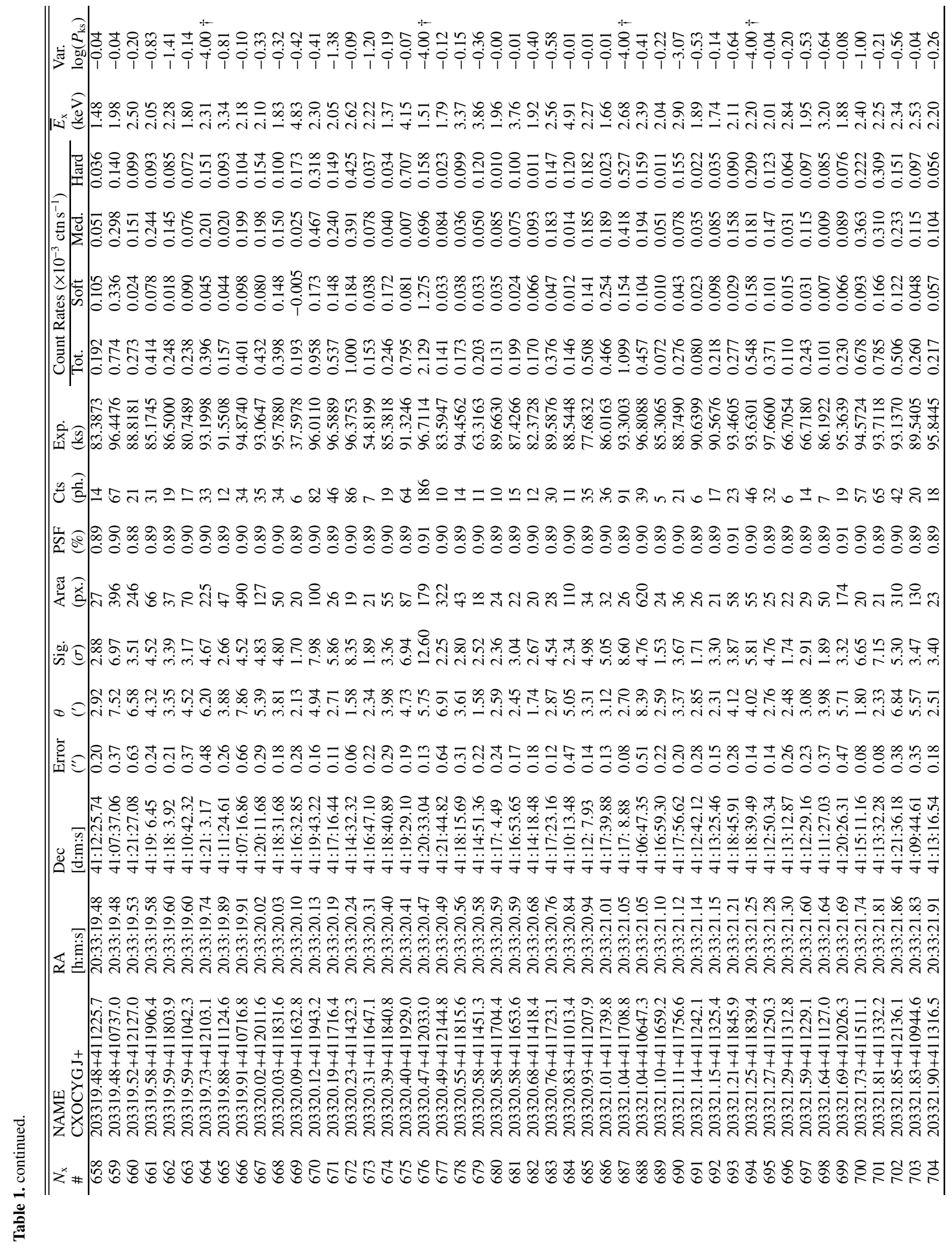


J. F. Albacete Colombo et al.: Unveiling the Cygnus OB2 stellar population with Chandra, Online Material p 17

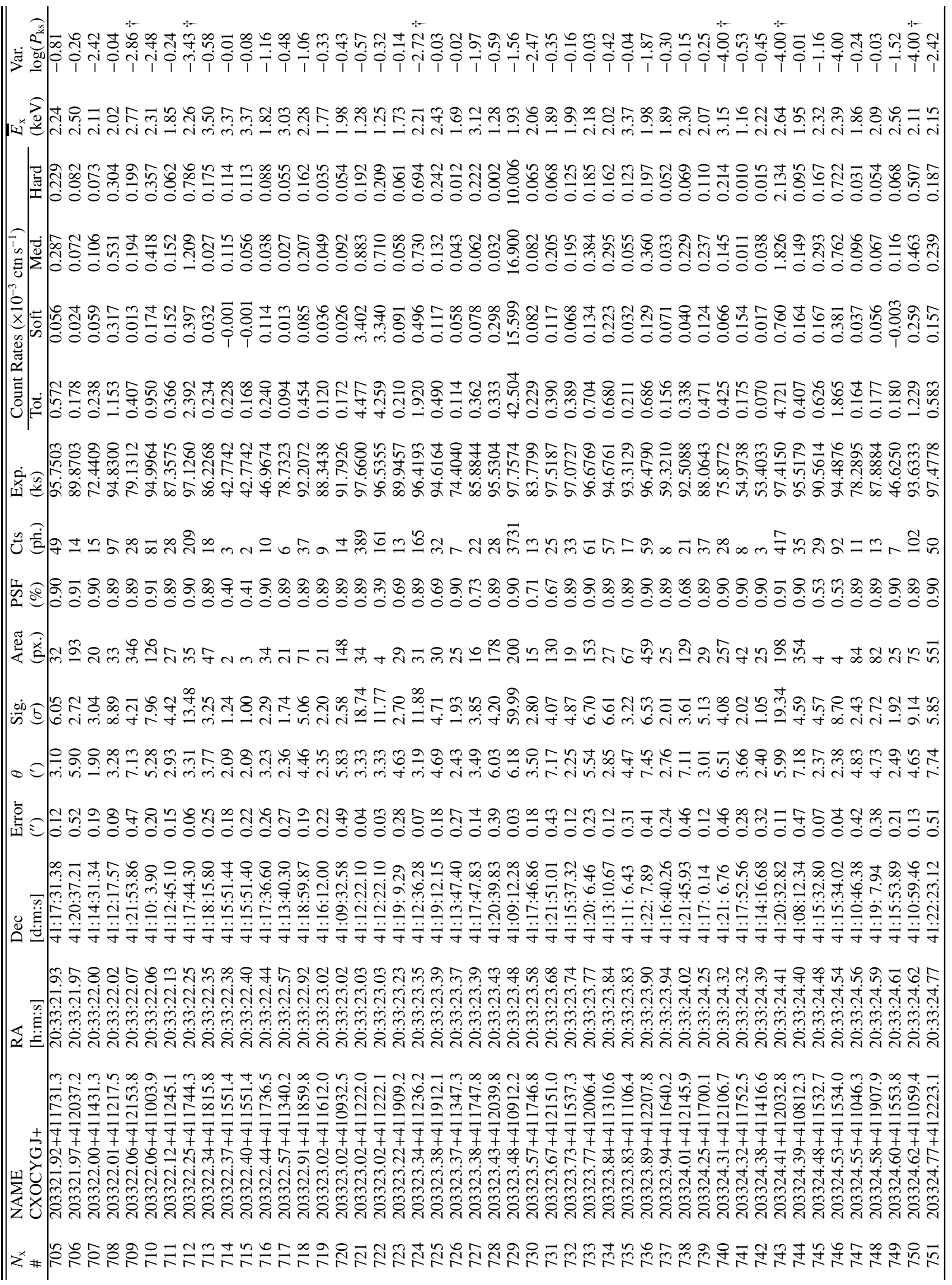


J. F. Albacete Colombo et al.: Unveiling the Cygnus OB2 stellar population with Chandra, Online Material p 18

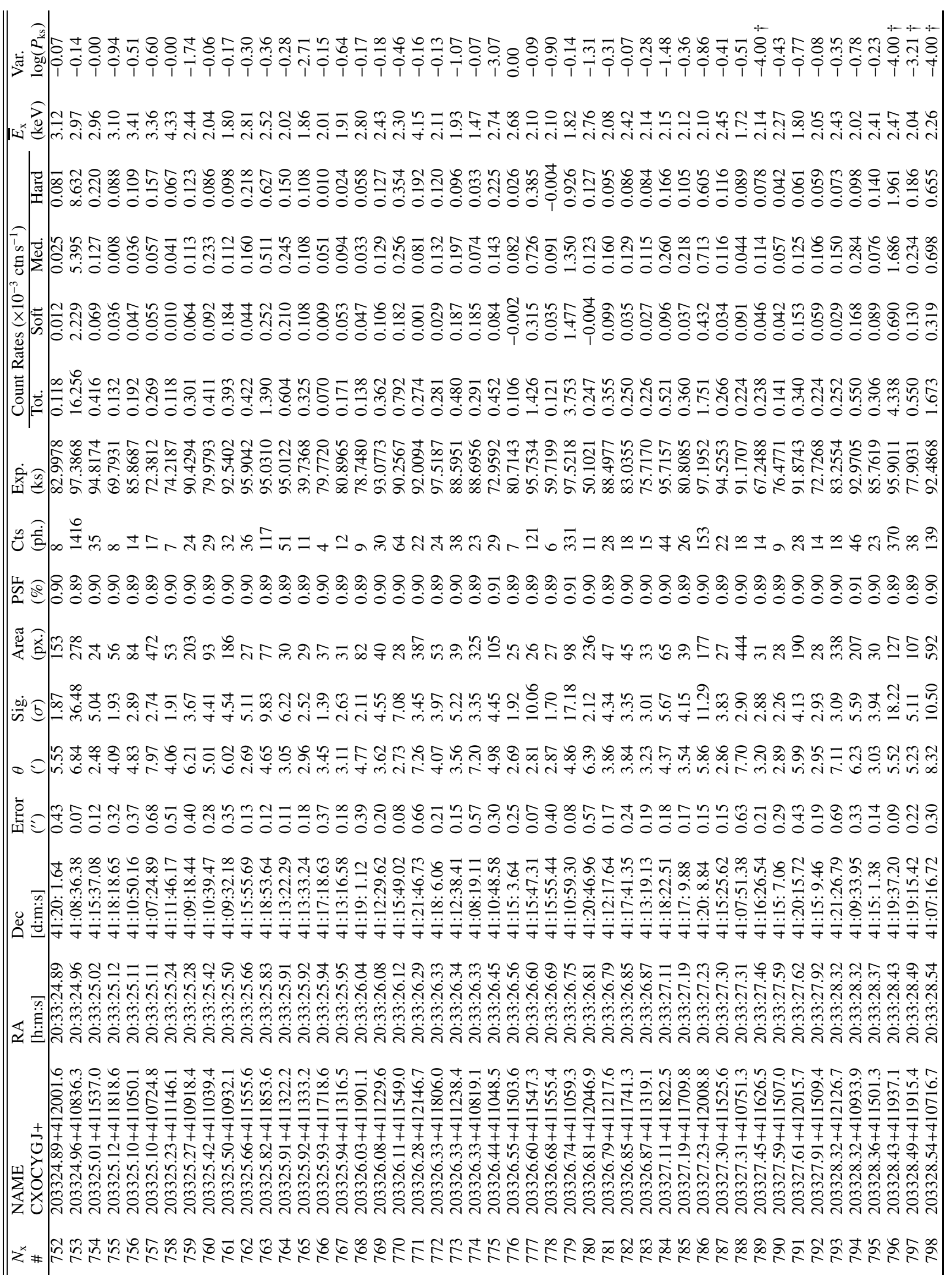


J. F. Albacete Colombo et al.: Unveiling the Cygnus OB2 stellar population with Chandra, Online Material p 19

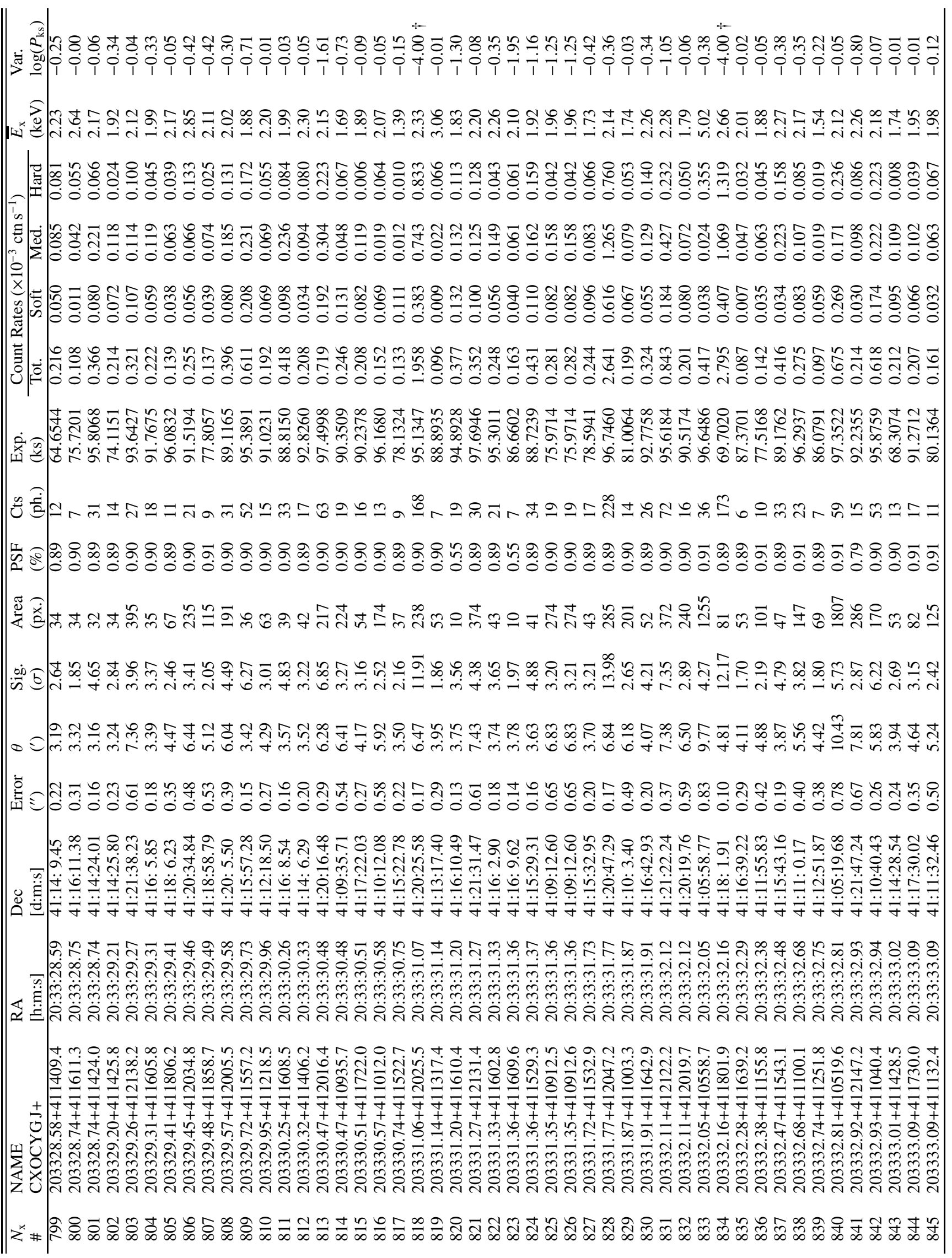


J. F. Albacete Colombo et al.: Unveiling the Cygnus OB2 stellar population with Chandra, Online Material p 20

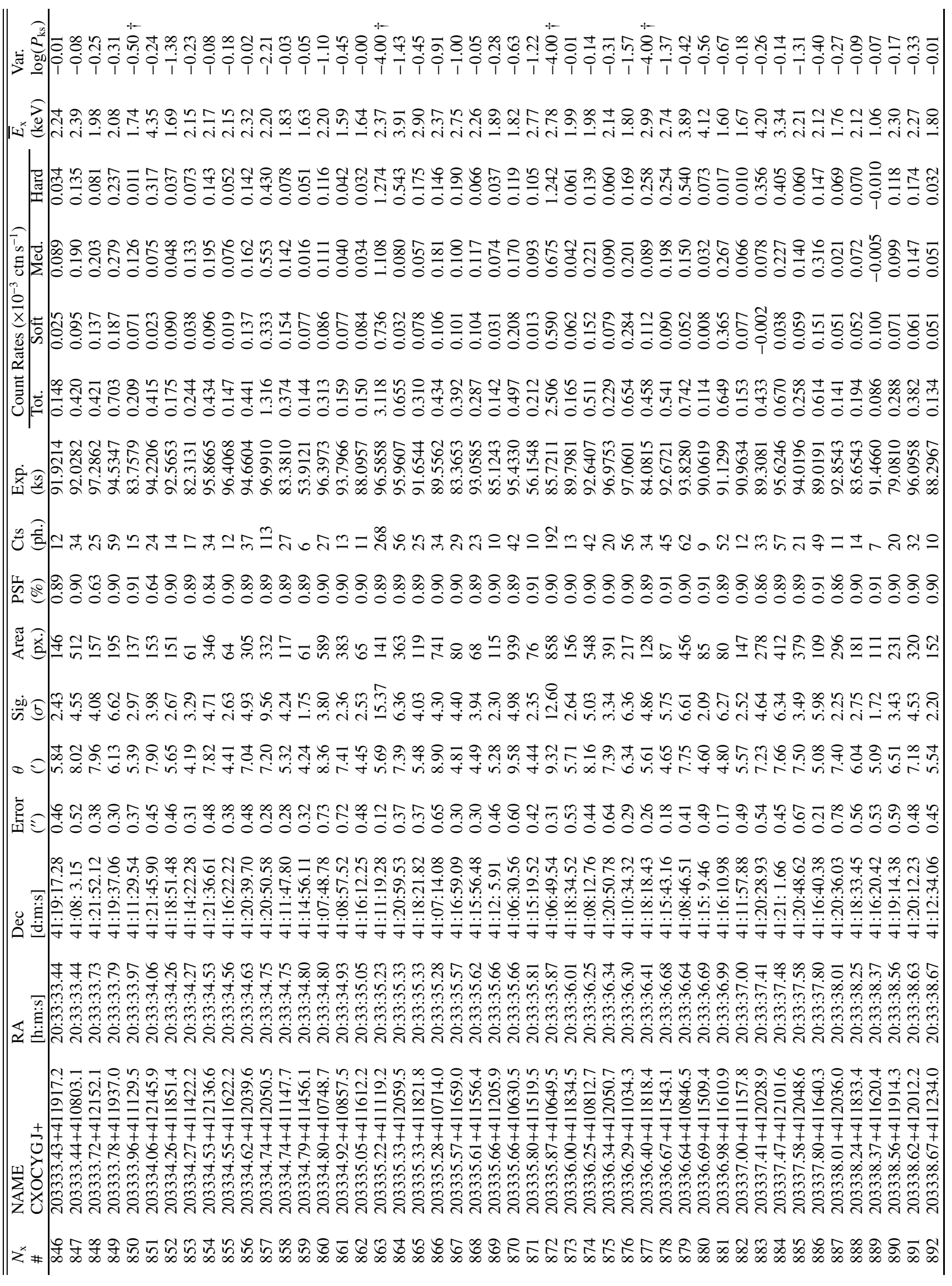


J. F. Albacete Colombo et al.: Unveiling the Cygnus OB2 stellar population with Chandra, Online Material p 21

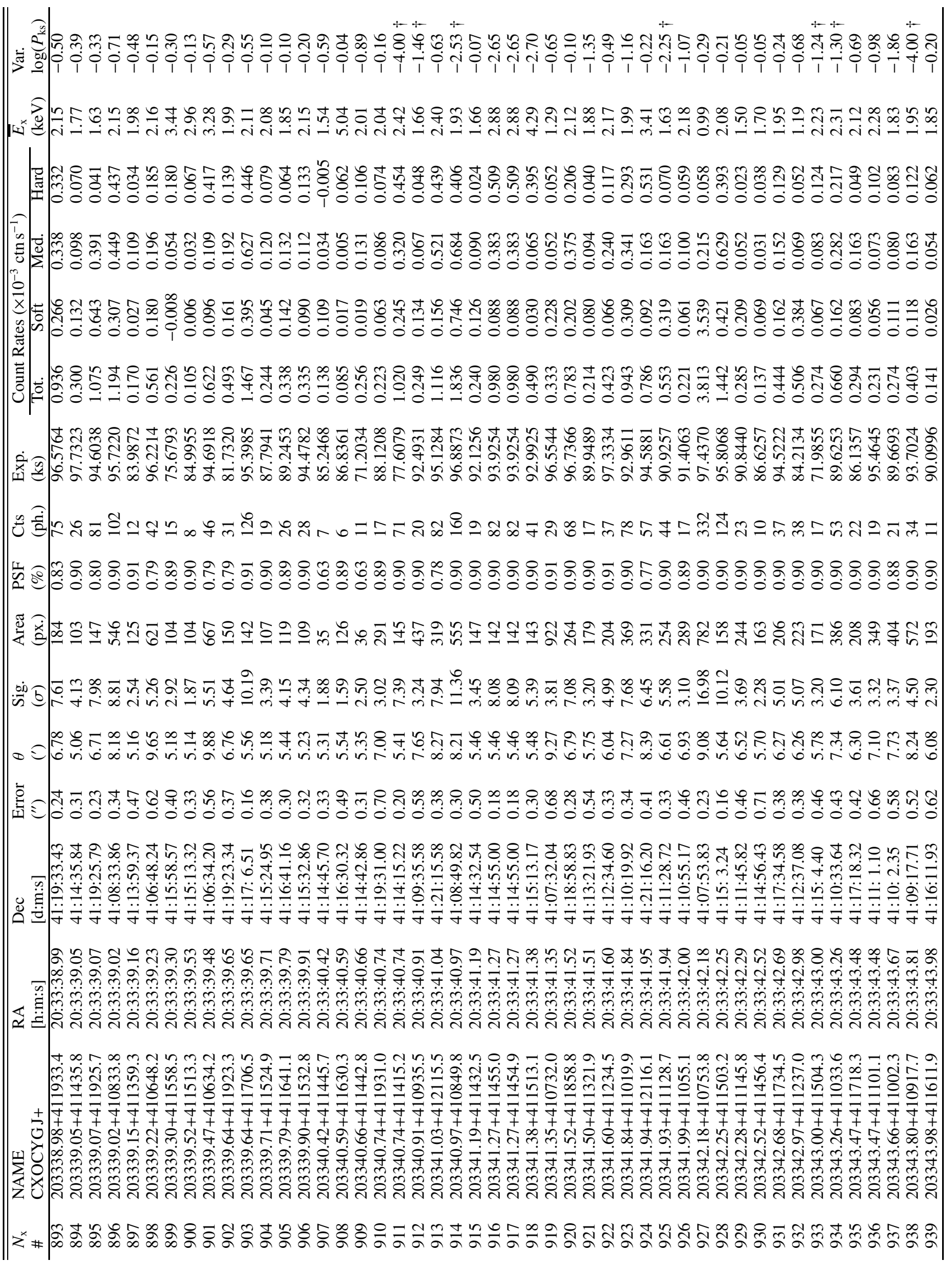


J. F. Albacete Colombo et al.: Unveiling the Cygnus OB2 stellar population with Chandra, Online Material p 22

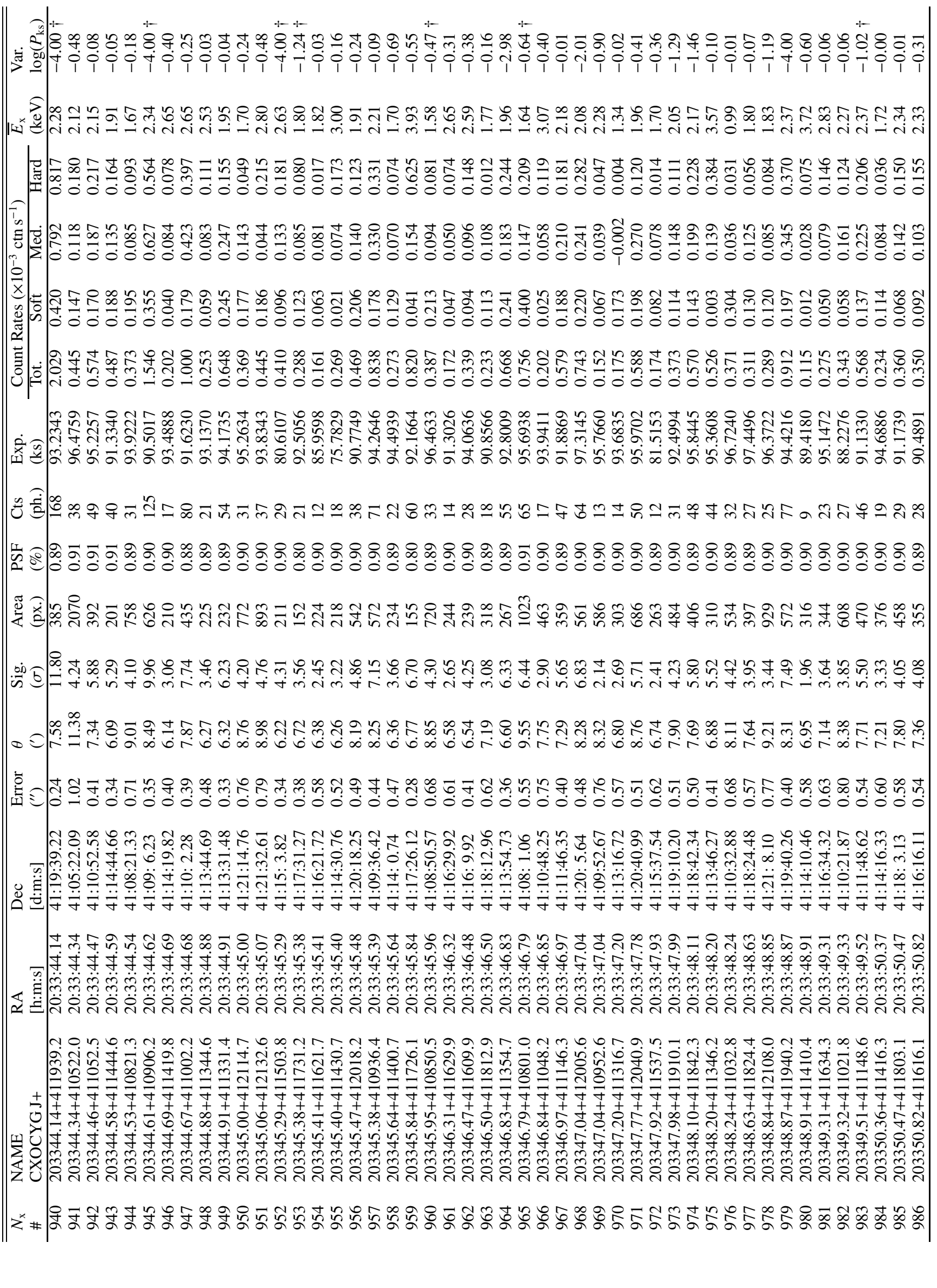




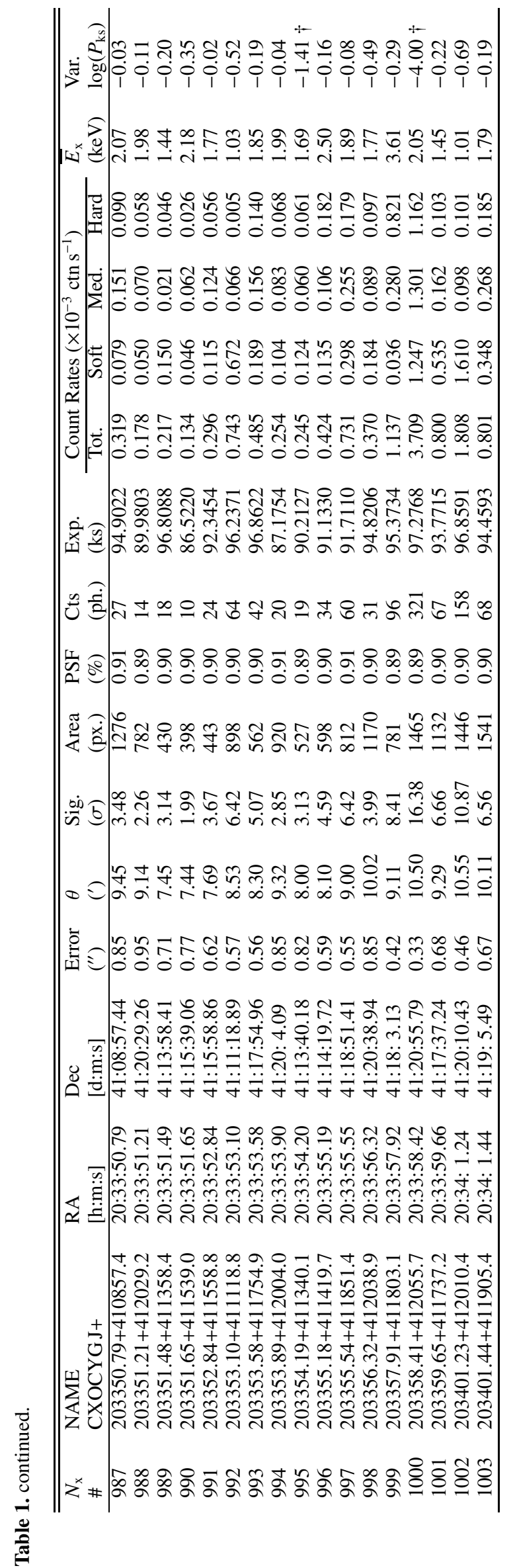


Table 2. Near-IR counterparts of Cygnus OB2 X-ray sources and near-IR stellar parameters.

\begin{tabular}{|c|c|c|c|c|c|c|c|c|}
\hline \multirow{2}{*}{$\begin{array}{l}N_{\mathrm{x}} \\
\#\end{array}$} & \multicolumn{2}{|c|}{ X-ray - 2MASS counterpart } & \multirow{2}{*}{$\begin{array}{l}\text { Off. } \\
\left({ }^{\prime \prime}\right)\end{array}$} & \multicolumn{4}{|c|}{ 2MASS photometry } & \multirow{2}{*}{$\begin{array}{l}A_{\mathrm{v}} \\
\text { (mag) }\end{array}$} \\
\hline & CXOAC J+ & 2MASS J+ & & $J$ & $H$ & $K_{\mathrm{s}}$ & Ph. qual & \\
\hline 1 & $203225.09+411019.6$ & - & - & - & - & - & - & - \\
\hline 2 & $203225.55+410847.3$ & $20322545+4108473$ & 1.56 & $14.76 \pm 0.06$ & $14.23 \pm 0.07$ & $13.95 \pm 0.07$ & AAA & 8.54 \\
\hline 3 & $203225.97+411054.7$ & $20322597+4110547$ & 0.24 & $15.24 \pm 0.05$ & $13.65 \pm 0.03$ & $12.69 \pm 0.03$ & AAA & 7.71 \\
\hline 4 & $203227.51+411358.3$ & $20322756+4114001$ & 1.61 & $16.00 \pm 0.06$ & $14.46 \pm 0.04$ & $13.90 \pm 0.06$ & AAA & 0.16 \\
\hline 5 & $203227.62+410831.4$ & $20322754+4108336$ & 2.41 & $15.91 \pm 0.06$ & $14.39 \pm 0.03$ & $13.82 \pm 0.05$ & AAA & N/A \\
\hline 6 & $203227.70+411317.0$ & $20322768+4113169$ & 0.58 & $14.96 \pm 0.03$ & $13.49 \pm 0.02$ & $12.88 \pm 0.03$ & AAA & 2.21 \\
\hline 7 & $203228.88+410807.5$ & - & - & - & - & - & - & - \\
\hline 8 & $203229.12+411400.7$ & $20322913+4114012$ & 0.26 & $16.74 \pm$ & $15.36 \pm 0.08$ & $14.72 \pm 0.11$ & CAB & 9.01 \\
\hline 9 & $203229.26+410850.1$ & $20322928+4108494$ & 1.03 & $12.26 \pm 0.01$ & $11.49 \pm 0.01$ & $11.07 \pm 0.01$ & AAA & 8.52 \\
\hline 10 & $203229.84+411454.2$ & $20322985+4114536$ & 0.83 & 17.44 & $15.43 \pm 0.10$ & $14.37 \pm 0.09$ & UAA & N/A \\
\hline 11 & $203229.91+411056.0$ & $20322994+4110575$ & 1.29 & $15.26 \pm 0.05$ & $14.04 \pm 0.04$ & $13.53 \pm 0.04$ & AAA & N/A \\
\hline 12 & $203230.63+410829.1$ & $20323051+4108284$ & 2.19 & $15.47 \pm 0.07$ & 13.71 & 13.09 & AUU & 4.19 \\
\hline 13 & $203230.63+410854.8$ & $20323064+4108565$ & 1.49 & $15.31 \pm 0.05$ & $13.68 \pm 0.03$ & $13.01 \pm 0.03$ & AAA & 5.43 \\
\hline 14 & $203230.88+411029.5$ & - & - & - & - & - & - & - \\
\hline 15 & $203231.39+410955.9$ & $20323143+4109558$ & 0.58 & $14.52 \pm 0.05$ & 13.04 & 12.46 & AUU & 9.69 \\
\hline 16 & $203231.42+411335.0$ & - & - & - & - & - & - & - \\
\hline 17 & $203231.53+411407.9$ & $20323154+4114082$ & 0.11 & $7.82 \pm 0.01$ & $7.09 \pm 0.02$ & $6.66 \pm 0.01$ & AAA & 4.66 \\
\hline 18 & $203231.58+411712.5$ & - & - & - & - & - & - & - \\
\hline 19 & $203232.32+411507.3$ & $20323242+4115082$ & 1.70 & $16.41 \pm 0.10$ & $14.62 \pm 0.05$ & $13.69 \pm 0.05$ & BAA & 4.87 \\
\hline 20 & $203232.44+411312.3$ & $20323249+4113131$ & 0.80 & $14.29 \pm 0.02$ & $13.25 \pm 0.02$ & $12.70 \pm 0.03$ & AAA & 9.17 \\
\hline 21 & $203233.28+411058.8$ & $20323346+4110594$ & 2.65 & $11.70 \pm 0.01$ & $11.50 \pm 0.02$ & $11.44 \pm 0.02$ & AAA & 10.45 \\
\hline 22 & $203233.47+411218.0$ & $20323349+4112184$ & 0.19 & $13.48 \pm 0.02$ & $12.49 \pm 0.02$ & $12.00 \pm 0.02$ & AAA & 8.33 \\
\hline 23 & $203233.53+411405.7$ & $20323351+4114056$ & 0.63 & $13.28 \pm 0.02$ & $12.48 \pm 0.01$ & $12.09 \pm 0.02$ & AAA & N/A \\
\hline 24 & $203234.33+411506.3$ & - & - & - & - & - & - & - \\
\hline 25 & $203234.45+411154.7$ & $20323447+4111552$ & 0.39 & $14.69 \pm 0.03$ & $13.26 \pm 0.02$ & $12.68 \pm 0.03$ & AAA & 7.21 \\
\hline 26 & $203235.17+411143.4$ & $20323518+4111432$ & 0.45 & $14.85 \pm 0.03$ & $13.47 \pm 0.02$ & $12.97 \pm 0.03$ & AAA & 7.73 \\
\hline 27 & $203235.65+411509.5$ & $20323564+4115097$ & 0.33 & $10.85 \pm 0.02$ & $10.19 \pm 0.02$ & $9.85 \pm 0.03$ & AAA & N/A \\
\hline 28 & $203235.86+411248.4$ & $20323592+4112505$ & 2.08 & $15.81 \pm 0.07$ & 13.58 & 12.95 & AUU & 4.18 \\
\hline 29 & $203235.96+412135.6$ & $20323599+4121363$ & 0.55 & $15.08 \pm 0.03$ & $13.99 \pm 0.02$ & $13.53 \pm 0.04$ & AAA & N/A \\
\hline 30 & $203236.32+411900.7$ & $20323641+4119010$ & 1.32 & $15.96 \pm 0.09$ & $14.63 \pm 0.08$ & $14.12 \pm 0.08$ & AAA & N/A \\
\hline 31 & $203236.38+411831.0$ & $20323629+4118308$ & 1.51 & $15.52 \pm 0.06$ & $14.35 \pm 0.07$ & $13.90 \pm 0.05$ & AAA & N/A \\
\hline 32 & $203236.50+411810.3$ & $20323643+4118103$ & 1.13 & $16.24 \pm 0.09$ & $14.96 \pm 0.07$ & $14.77 \pm 0.12$ & AAB & 9.19 \\
\hline 33 & $203236.62+412214.5$ & $20323661+4122147$ & 0.22 & $12.11 \pm 0.02$ & $11.34 \pm 0.01$ & $10.88 \pm 0.02$ & AAA & N/A \\
\hline 34 & $203236.86+411944.6$ & $20323680+4119447$ & 1.00 & $15.45 \pm 0.06$ & $14.23 \pm 0.05$ & $13.76 \pm 0.06$ & AAA & 10.11 \\
\hline 35 & $203236.84+412146.1$ & - & - & - & - & - & - & - \\
\hline 36 & $203237.55+411150.8$ & $20323754+4111514$ & 0.39 & $13.34 \pm 0.02$ & $12.82 \pm 0.02$ & $12.64 \pm 0.03$ & AAA & 9.77 \\
\hline 37 & $203237.53+411712.4$ & $20323751+4117121$ & 0.65 & 17.36 & $15.86 \pm 0.13$ & 15.83 & UBU & 19.97 \\
\hline 38 & $203237.71+411405.3$ & $20323767+4114052$ & 0.86 & $13.42 \pm 0.02$ & $12.16 \pm 0.02$ & $11.61 \pm 0.02$ & AAA & 6.76 \\
\hline 39 & $203237.83+412208.7$ & $20323784+4122087$ & 0.20 & $15.16 \pm 0.04$ & $14.07 \pm 0.04$ & $13.65 \pm 0.05$ & AAA & 17.08 \\
\hline 40 & $203237.87+412119.0$ & $20323787+4121194$ & 0.19 & $14.37 \pm 0.02$ & $13.21 \pm 0.02$ & $12.81 \pm 0.03$ & AAA & 6.26 \\
\hline 41 & $203237.94+411502.7$ & $20323781+4115026$ & 2.11 & $15.05 \pm 0.05$ & $14.01 \pm 0.04$ & $13.47 \pm 0.05$ & AAA & N/A \\
\hline 42 & $203238.10+410833.1$ & $20323804+4108316$ & 1.94 & $15.57 \pm 0.05$ & $14.08 \pm 0.03$ & $13.43 \pm 0.04$ & AAA & N/A \\
\hline 43 & $203238.10+411244.4$ & $20323810+4112442$ & 0.49 & $15.45 \pm 0.06$ & $14.23 \pm 0.03$ & $13.64 \pm 0.05$ & AAA & 8.73 \\
\hline 44 & $203238.07+412313.8$ & $20323799+4123138$ & 1.25 & 13.76 & $12.78 \pm 0.03$ & 12.35 & UAU & 17.96 \\
\hline 45 & $203238.26+411141.7$ & $20323830+4111422$ & 0.49 & $16.04 \pm 0.08$ & $14.48 \pm 0.04$ & $13.96 \pm 0.06$ & AAA & 7.88 \\
\hline 46 & $203238.43+411201.1$ & - & - & - & - & - & - & - \\
\hline 47 & $203238.56+411422.0$ & - & - & - & - & - & - & - \\
\hline 48 & $203239.41+411906.3$ & $20323944+4119066$ & 0.22 & $14.95 \pm 0.04$ & $13.86 \pm 0.03$ & $13.56 \pm 0.04$ & AAA & 2.94 \\
\hline 49 & $203239.52+411112.5$ & $20323951+4111129$ & 0.17 & $14.98 \pm 0.03$ & $13.48 \pm 0.03$ & $12.90 \pm 0.03$ & AAA & 3.81 \\
\hline 50 & $203239.82+412358.2$ & $20323986+4123595$ & 1.21 & $14.78 \pm 0.03$ & $13.58 \pm 0.03$ & $13.18 \pm 0.03$ & AAA & 10.87 \\
\hline 51 & $203239.90+411348.1$ & $20323989+4113488$ & 0.52 & $16.49 \pm 0.14$ & $15.26 \pm 0.09$ & $15.05 \pm 0.20$ & BAC & 8.51 \\
\hline 52 & $203240.12+411034.7$ & $20324009+4110346$ & 0.52 & $11.78 \pm 0.02$ & $11.04 \pm 0.02$ & $10.64 \pm 0.01$ & AAA & N/A \\
\hline 53 & $203240.07+412201.7$ & $20324004+4122015$ & 0.74 & $16.10 \pm 0.09$ & $14.65 \pm 0.05$ & $14.26 \pm 0.08$ & AAA & 5.71 \\
\hline 54 & $203240.22+412244.7$ & $20324032+4122473$ & 2.64 & 15.49 & 14.33 & $14.37 \pm 0.09$ & UUA & 8.10 \\
\hline 55 & $203240.26+411740.9$ & $20324026+4117408$ & 0.29 & $15.63 \pm 0.06$ & $14.29 \pm 0.04$ & $13.86 \pm 0.06$ & AAA & 6.17 \\
\hline 56 & $203240.59+411454.8$ & $20324061+4114548$ & 0.23 & $15.35 \pm 0.18$ & 12.73 & 11.92 & CUU & 6.59 \\
\hline 57 & $203240.65+411111.6$ & $20324063+4111113$ & 0.69 & $16.58 \pm 0.13$ & $14.81 \pm 0.06$ & $14.23 \pm 0.08$ & BAA & 5.18 \\
\hline 58 & $203240.72+412106.1$ & $20324067+4121059$ & 0.99 & $14.81 \pm 0.04$ & $13.73 \pm 0.04$ & $13.39 \pm 0.04$ & AAA & 5.91 \\
\hline 59 & $203240.81+411720.2$ & $20324077+4117208$ & 0.86 & $15.41 \pm 0.05$ & $14.20 \pm 0.06$ & $13.82 \pm 0.06$ & AAA & 7.40 \\
\hline 60 & $203240.94+411428.8$ & $20324096+4114291$ & 0.11 & $4.67 \pm 0.32$ & $3.51 \pm 0.26$ & $2.70 \pm 0.36$ & DDD & N/A \\
\hline 61 & $203240.96+411551.0$ & $20324094+4115511$ & 0.47 & $13.47 \pm 0.02$ & $12.65 \pm 0.02$ & $12.18 \pm 0.02$ & AAA & 1.34 \\
\hline 62 & $203240.97+411449.6$ & - & - & - & - & - & - & - \\
\hline 63 & $203241.06+411955.2$ & $20324109+4119552$ & 0.40 & $14.51 \pm 0.03$ & $13.54 \pm 0.03$ & $13.16 \pm 0.04$ & AAA & 12.07 \\
\hline 64 & $203241.33+411849.9$ & $20324133+4118501$ & 0.23 & $12.95 \pm 0.02$ & $12.01 \pm 0.02$ & $11.60 \pm 0.02$ & AAA & 7.06 \\
\hline
\end{tabular}


Table 2. continued.

\begin{tabular}{|c|c|c|c|c|c|c|c|c|}
\hline \multirow{2}{*}{$\begin{array}{l}N_{\mathrm{x}} \\
\#\end{array}$} & \multicolumn{2}{|c|}{ X-ray - 2MASS counterpart } & \multirow{2}{*}{$\begin{array}{l}\text { Off. } \\
\left({ }^{\prime \prime}\right)\end{array}$} & \multicolumn{4}{|c|}{ 2MASS photometry } & \multirow{2}{*}{$\begin{array}{l}\begin{array}{l}A_{\mathrm{v}} \\
(\mathrm{mag})\end{array} \\
\text { (a) }\end{array}$} \\
\hline & CXOAC J+ & 2MASS J+ & & $\bar{J}$ & $H$ & $K_{\mathrm{s}}$ & Ph.qual & \\
\hline 65 & $203241.55+411154.7$ & $\overline{-}$ & - & - & - & - & - & - \\
\hline 66 & $203241.61+411549.0$ & $20324158+4115486$ & 0.78 & 15.89 & 14.73 & $14.96 \pm 0.15$ & UUB & 5.50 \\
\hline 67 & $203241.66+411406.5$ & - & - & - & - & - & - & - \\
\hline 68 & $203241.87+411555.9$ & $20324185+4115557$ & 0.53 & $16.24 \pm 0.10$ & $14.82 \pm 0.06$ & $14.30 \pm 0.09$ & AAA & $\mathrm{N} / \mathrm{A}$ \\
\hline 69 & $203242.15+411712.2$ & $20324210+4117122$ & 0.91 & $16.69 \pm 0.13$ & $15.42 \pm 0.09$ & $14.81 \pm 0.14$ & BAB & 5.47 \\
\hline 70 & $203242.18+411641.4$ & $20324216+4116412$ & 0.65 & $15.30 \pm 0.05$ & $14.02 \pm 0.04$ & $13.61 \pm 0.05$ & AAA & 6.83 \\
\hline 71 & $203242.46+412236.6$ & $20324235+4122360$ & 1.94 & $14.58 \pm 0.03$ & $13.48 \pm 0.03$ & $13.10 \pm 0.04$ & AAA & 4.50 \\
\hline 72 & $203242.88+412016.5$ & $20324289+4120164$ & 0.36 & $10.68 \pm 0.02$ & $10.21 \pm 0.01$ & $10.00 \pm 0.01$ & AAA & N/A \\
\hline 73 & $203242.86+412343.1$ & $20324290+4123437$ & 0.55 & $13.01 \pm 0.02$ & $12.14 \pm 0.02$ & $11.82 \pm 0.02$ & AAA & 7.64 \\
\hline 74 & $203242.91+411914.4$ & $20324288+4119148$ & 0.58 & $15.03 \pm 0.04$ & $13.82 \pm 0.04$ & $13.37 \pm 0.04$ & AAA & 6.01 \\
\hline 75 & $203243.03+411741.1$ & $20324313+4117417$ & 1.39 & 15.65 & $14.66 \pm 0.06$ & 14.02 & UAU & 7.77 \\
\hline 76 & $203243.01+412309.3$ & $20324298+4123107$ & 1.31 & $14.90 \pm 0.03$ & $13.74 \pm 0.03$ & $13.32 \pm 0.04$ & AAA & N/A \\
\hline 77 & $203243.09+411359.3$ & $20324307+4113594$ & 0.35 & $15.26 \pm 0.04$ & $13.71 \pm 0.04$ & $13.39 \pm 0.04$ & AAA & 1.09 \\
\hline 78 & $203243.10+411750.9$ & - & - & - & - & - & - & - \\
\hline 79 & $203243.34+411514.1$ & - & - & - & - & - & - & - \\
\hline 80 & $203243.34+411657.1$ & - & - & - & - & - & - & - \\
\hline 81 & $203243.40+411102.9$ & $20324342+4111034$ & 0.31 & $16.44 \pm 0.11$ & $14.78 \pm 0.06$ & $14.24 \pm 0.08$ & BAA & 8.43 \\
\hline 82 & $203243.95+411709.9$ & $20324393+4117101$ & 0.33 & $16.61 \pm 0.14$ & $15.32 \pm 0.09$ & $14.70 \pm 0.12$ & BAB & N/A \\
\hline 83 & $203244.22+411615.7$ & $20324426+4116162$ & 0.53 & $16.39 \pm 0.10$ & $15.03 \pm 0.06$ & $14.59 \pm 0.11$ & BAA & 12.29 \\
\hline 84 & $203244.31+411743.8$ & $20324427+4117436$ & 0.78 & $16.02 \pm 0.08$ & $14.75 \pm 0.08$ & $14.18 \pm 0.08$ & AAA & N/A \\
\hline 85 & $203244.35+411445.3$ & $20324431+4114453$ & 0.81 & $14.85 \pm 0.03$ & $13.45 \pm 0.01$ & $12.91 \pm 0.03$ & AAA & 1.97 \\
\hline 86 & $203244.42+411122.9$ & $20324443+4111236$ & 0.51 & $12.56 \pm 0.01$ & $11.69 \pm 0.01$ & $11.25 \pm 0.01$ & AAA & N/A \\
\hline 87 & $203244.71+410701.4$ & $20324468+4106596$ & 2.11 & $14.16 \pm 0.02$ & $12.89 \pm 0.02$ & $12.35 \pm 0.02$ & AAA & 5.27 \\
\hline 88 & $203244.74+411305.4$ & $20324475+4113057$ & 0.02 & $12.10 \pm 0.02$ & $11.23 \pm 0.01$ & $10.81 \pm 0.02$ & AAA & N/A \\
\hline 89 & $203244.81+411227.4$ & $20324480+4112281$ & 0.51 & $13.57 \pm 0.02$ & $12.27 \pm 0.01$ & $11.59 \pm 0.02$ & AAA & 8.51 \\
\hline 90 & $203244.82+411549.0$ & $20324480+4115484$ & 0.98 & $15.79 \pm 0.08$ & $14.49 \pm 0.07$ & $14.06 \pm 0.07$ & AAA & N/A \\
\hline 91 & $203244.85+411419.1$ & - & - & - & - & - & - & - \\
\hline 92 & $203244.83+411848.1$ & $20324484+4118484$ & 0.09 & $15.45 \pm 0.06$ & $14.16 \pm 0.05$ & $13.81 \pm 0.05$ & AAA & 8.30 \\
\hline 93 & $203244.90+411519.8$ & $20324492+4115203$ & 0.33 & $14.08 \pm 0.03$ & $12.64 \pm 0.02$ & $12.00 \pm 0.02$ & AAA & 6.55 \\
\hline 94 & $203244.86+412350.5$ & $20324478+4123519$ & 1.72 & $12.74 \pm 0.02$ & $12.05 \pm 0.02$ & $11.72 \pm 0.02$ & AAA & $\mathrm{N} / \mathrm{A}$ \\
\hline 95 & $203245.08+411247.3$ & $20324510+4112485$ & 0.99 & $16.29 \pm 0.10$ & $14.92 \pm 0.06$ & $14.15 \pm 0.07$ & AAA & 18.57 \\
\hline 96 & $203245.08+411725.6$ & - & - & - & - & - & - & - \\
\hline 97 & $203245.24+411221.2$ & $20324523+4112209$ & 0.59 & $16.06 \pm 0.08$ & $14.51 \pm 0.04$ & $13.65 \pm 0.05$ & AAA & 7.34 \\
\hline 98 & $203245.30+411721.1$ & $20324529+4117209$ & 0.51 & 16.77 & $15.41 \pm 0.10$ & 14.59 & UAU & 8.24 \\
\hline 99 & $203245.49+411546.9$ & $20324542+4115465$ & 1.28 & $15.48 \pm 0.05$ & $14.16 \pm 0.05$ & $13.68 \pm 0.05$ & AAA & 9.18 \\
\hline 100 & $203245.59+411659.2$ & - & - & - & - & - & - & - \\
\hline 101 & $203245.64+411647.1$ & $20324569+4116473$ & 0.65 & $16.05 \pm 0.08$ & $14.47 \pm 0.04$ & $13.66 \pm 0.05$ & AAA & N/A \\
\hline 102 & $203245.76+411349.7$ & $20324577+4113497$ & 0.16 & $15.10 \pm 0.05$ & $13.66 \pm 0.06$ & $13.18 \pm 0.05$ & AAA & 9.22 \\
\hline 103 & $203245.81+411502.9$ & $20324583+4115028$ & 0.36 & $15.75 \pm 0.06$ & $14.48 \pm 0.05$ & $14.13 \pm 0.07$ & AAA & 5.21 \\
\hline 104 & $203245.79+412108.2$ & $20324580+4121083$ & 0.08 & $12.18 \pm 0.02$ & $11.53 \pm 0.02$ & $11.21 \pm 0.01$ & AAA & 3.24 \\
\hline 105 & $203245.91+411238.7$ & $20324589+4112383$ & 0.76 & $15.41 \pm 0.05$ & $14.14 \pm 0.03$ & $13.70 \pm 0.05$ & AAA & N/A \\
\hline 106 & $203246.14+411040.1$ & $20324624+4110396$ & 1.70 & $14.57 \pm 0.04$ & $12.91 \pm 0.03$ & 11.10 & AAU & 15.25 \\
\hline 107 & $203246.27+410713.3$ & $20324630+4107141$ & 0.74 & $14.62 \pm 0.03$ & $13.42 \pm 0.03$ & $12.93 \pm 0.03$ & AAA & N/A \\
\hline 108 & $203246.31+411107.8$ & $20324634+4111076$ & 0.53 & $11.74 \pm 0.02$ & $11.07 \pm 0.02$ & $10.71 \pm 0.02$ & AAA & 8.01 \\
\hline 109 & $203246.36+411652.9$ & $20324651+4116534$ & 2.10 & $15.01 \pm 0.04$ & $14.35 \pm 0.05$ & $14.15 \pm 0.08$ & AAA & 4.54 \\
\hline 110 & $203246.41+411301.8$ & - & - & - & - & - & - & - \\
\hline 111 & $203246.66+411808.5$ & $20324666+4118085$ & 0.23 & $16.34 \pm 0.10$ & $14.87 \pm 0.06$ & $14.47 \pm 0.09$ & AAA & 2.43 \\
\hline 112 & $203246.70+411316.3$ & - & - & - & - & - & - & - \\
\hline 113 & $203246.86+411742.8$ & $20324681+4117442$ & 1.48 & $16.15 \pm 0.11$ & 13.84 & 13.30 & BUU & 6.14 \\
\hline 114 & $203246.91+412118.7$ & $20324678+4121197$ & 2.24 & $16.16 \pm 0.13$ & $14.96 \pm 0.10$ & $14.55 \pm 0.11$ & BAA & 7.89 \\
\hline 115 & $203247.40+411251.1$ & $20324744+4112513$ & 0.44 & $15.68 \pm 0.05$ & $14.13 \pm 0.04$ & $13.54 \pm 0.04$ & AAA & 1.29 \\
\hline 116 & $203247.47+411211.7$ & - & - & - & - & - & - & - \\
\hline 117 & $203247.81+411334.8$ & $20324778+4113363$ & 1.37 & $16.38 \pm$ & $15.18 \pm$ & $14.73 \pm 0.12$ & BAB & 3.72 \\
\hline 118 & $203247.87+411632.3$ & $20324781+4116324$ & 1.01 & $15.37 \pm 0.04$ & $14.11 \pm 0.03$ & $13.47 \pm 0.04$ & AAA & 5.52 \\
\hline 119 & $203247.98+411653.9$ & $20324801+4116542$ & 0.33 & $16.10 \pm 0.08$ & $14.72 \pm 0.06$ & $14.36 \pm 0.09$ & AAA & N/A \\
\hline 120 & $203248.18+411352.1$ & - & - & - & - & - & - & - \\
\hline 121 & $203248.25+411229.1$ & - & - & - & - & - & - & - \\
\hline 122 & $203248.43+411439.4$ & $20324844+4114396$ & 0.06 & $15.99 \pm($ & $14.55 \pm 0.07$ & $13.96 \pm$ & AAA & 4.81 \\
\hline 123 & $203248.47+411608.2$ & $20324845+4116080$ & 0.57 & $12.25 \pm 0.02$ & $11.67 \pm 0.02$ & $11.32 \pm 0.02$ & AAA & 5.69 \\
\hline 124 & $203248.53+412336.2$ & $20324844+4123366$ & 1.45 & $14.23 \pm 0.02$ & $13.14 \pm 0.02$ & $12.78 \pm 0.03$ & AAA & N/A \\
\hline 125 & $203248.63+410811.6$ & $20324860+4108117$ & 0.45 & $15.34 \pm 0.05$ & $13.97 \pm 0.03$ & $13.51 \pm 0.04$ & AAA & 8.14 \\
\hline 126 & $203248.87+411519.8$ & $20324891+4115197$ & 0.54 & $14.81 \pm 0.03$ & $13.55 \pm 0.02$ & $12.97 \pm 0.03$ & AAA & 8.46 \\
\hline 127 & $203248.91+410930.4$ & - & - & - & - & - & - & - \\
\hline 128 & $203249.02+410746.8$ & $20324901+4107$ & 0.88 & $15.81 \pm 0.06$ & $14.40 \pm 0.05$ & $13.85 \pm 0.05$ & AAA & N/A \\
\hline 129 & $203249.39+411230.6$ & $20324937+4112307$ & 0.37 & $16.10 \pm 0.09$ & $14.99 \pm 0.06$ & $14.41 \pm 0.09$ & AAA & 6.50 \\
\hline 130 & $203249.38+411606.8$ & $20324938+4116072$ & 0.23 & $16.62 \pm 0.12$ & $15.36 \pm 0.10$ & $14.66 \pm 0.11$ & BAB & 11.75 \\
\hline
\end{tabular}


Table 2. continued.

\begin{tabular}{|c|c|c|c|c|c|c|c|c|}
\hline \multirow{2}{*}{$\begin{array}{l}N_{\mathrm{x}} \\
\#\end{array}$} & \multicolumn{2}{|c|}{$\overline{\mathrm{X} \text {-ray - 2MASS counterpart }}$} & \multirow{2}{*}{$\begin{array}{l}\text { Off. } \\
\left({ }^{\prime \prime}\right)\end{array}$} & \multicolumn{4}{|c|}{ 2MASS photometry } & \multirow{2}{*}{$\begin{array}{l}A_{\mathrm{v}} \\
(\mathrm{mag})\end{array}$} \\
\hline & CXOAC J+ & 2MASS J+ & & $J$ & $H$ & $K_{\mathrm{s}}$ & Ph.qual & \\
\hline 131 & $203249.34+412224.5$ & $20324939+4122247$ & 0.66 & $14.98 \pm 0.04$ & $13.85 \pm 0.04$ & $13.40 \pm 0.04$ & AAA & $\mathrm{N} / \mathrm{A}$ \\
\hline 132 & $203249.51+411532.8$ & $20324954+4115329$ & 0.24 & $13.48 \pm 0.02$ & $12.67 \pm 0.02$ & $12.25 \pm 0.02$ & AAA & 7.43 \\
\hline 133 & $203249.85+412342.2$ & - & - & - & - & - & - & - \\
\hline 134 & $203249.92+411511.1$ & $20324998+4115117$ & 0.83 & $16.04 \pm 0.07$ & $14.69 \pm 0.04$ & $14.11 \pm 0.07$ & AAA & 8.60 \\
\hline 135 & $203250.08+411626.7$ & $20325011+4116270$ & 0.33 & $16.62 \pm 0.12$ & $15.31 \pm 0.09$ & 13.56 & BAU & 4.27 \\
\hline 136 & $203250.11+411458.1$ & $20325012+4114586$ & 0.28 & $15.94 \pm 0.17$ & $14.39 \pm 0.10$ & $13.57 \pm 0.06$ & CAA & N/A \\
\hline 137 & $203250.16+411836.1$ & $20325014+4118362$ & 0.45 & $15.46 \pm 0.05$ & $14.32 \pm 0.04$ & 13.80 & AAU & 3.45 \\
\hline 138 & $203250.25+412216.6$ & - & - & - & - & - & - & - \\
\hline 139 & $203250.39+411038.2$ & $20325037+4110378$ & 0.67 & $16.21 \pm 0.09$ & $14.90 \pm 0.06$ & $14.41 \pm 0.09$ & AAA & N/A \\
\hline 140 & $203250.41+411749.5$ & $20325043+4117497$ & 0.22 & $15.06 \pm 0.04$ & $13.61 \pm 0.03$ & $13.13 \pm 0.04$ & AAA & N/A \\
\hline 141 & $203250.42+411629.6$ & $20325039+4116296$ & 0.58 & $14.77 \pm 0.03$ & $13.57 \pm 0.03$ & 13.01 & AAU & N/A \\
\hline 142 & $203250.69+410825.3$ & $20325069+4108258$ & 0.34 & $10.62 \pm 0.02$ & $9.89 \pm 0.01$ & $9.52 \pm 0.01$ & AAA & 7.23 \\
\hline 143 & $203250.69+411609.1$ & - & - & - & - & - & - & - \\
\hline 144 & $203250.87+411145.6$ & $20325089+4111461$ & 0.39 & 13.30 & $12.74 \pm 0.04$ & $12.34 \pm 0.03$ & UAA & 3.71 \\
\hline 145 & $203250.98+411245.6$ & - & - & - & - & - & - & - \\
\hline 146 & $203251.13+411253.5$ & - & - & - & - & - & - & - \\
\hline 147 & $203251.14+411237.7$ & $20325116+4112380$ & 0.11 & $15.45 \pm$ & $14.15 \pm 0.04$ & $13.65 \pm 0.05$ & AAA & 9.33 \\
\hline 148 & $203251.15+412026.1$ & $20325114+4120253$ & 1.08 & 14.94 & 13.80 & $13.43 \pm 0.05$ & UUA & 6.03 \\
\hline 149 & $203251.15+412245.8$ & $20325136+4122470$ & 3.25 & 14.80 & $13.64 \pm 0.04$ & 13.32 & UAU & N/A \\
\hline 150 & $203251.35+411123.7$ & - & - & - & - & - & - & - \\
\hline 151 & $203251.34+411617.2$ & - & - & - & - & - & - & - \\
\hline 152 & $203251.36+411409.0$ & $20325136+4114092$ & 0.06 & $12.77 \pm 0.02$ & $11.96 \pm 0.02$ & $11.58 \pm 0.02$ & AAA & 8.54 \\
\hline 153 & $203251.55+411300.7$ & $20325156+4113010$ & 0.10 & $14.78 \pm 0.03$ & $13.97 \pm 0.04$ & $13.80 \pm 0.05$ & AAA & N/A \\
\hline 154 & $203251.52+412037.7$ & $20325156+4120379$ & 0.44 & $16.06 \pm 0.08$ & $14.66 \pm 0.06$ & $13.90 \pm 0.06$ & AAA & 17.45 \\
\hline 155 & $203251.70+411710.9$ & $20325177+4117129$ & 1.99 & $16.26 \pm 0.09$ & $15.19 \pm 0.08$ & 14.28 & AAU & 7.03 \\
\hline 156 & $203252.00+411600.7$ & - & - & - & - & - & - & - \\
\hline 157 & $203252.11+411313.5$ & $20325212+4113135$ & 0.26 & $16.76 \pm 0.14$ & $15.35 \pm 0.09$ & $14.63 \pm 0.11$ & $\mathrm{CAB}$ & 3.30 \\
\hline 158 & $203252.12+411828.6$ & $20325210+4118289$ & 0.46 & $15.51 \pm 0.05$ & $14.08 \pm 0.04$ & $13.63 \pm 0.05$ & AAA & N/A \\
\hline 159 & $203252.17+411815.7$ & $20325218+4118160$ & 0.16 & $13.13 \pm 0.02$ & $12.41 \pm 0.02$ & $12.05 \pm 0.02$ & AAA & 6.28 \\
\hline 160 & $203252.41+411029.8$ & $20325244+4110296$ & 0.48 & $13.51 \pm 0.02$ & $12.59 \pm 0.02$ & $12.20 \pm 0.02$ & AAA & N/A \\
\hline 161 & $203252.47+411541.3$ & $20325249+4115426$ & 1.14 & $16.57 \pm 0.13$ & $15.12 \pm 0.07$ & $14.57 \pm 0.10$ & BAA & N/A \\
\hline 162 & $203252.52+411736.5$ & - & - & - & - & - & - & - \\
\hline 163 & $203252.59+411255.2$ & - & - & - & - & - & - & - \\
\hline 164 & $203252.60+412102.5$ & $20325261+4121027$ & 0.12 & $15.35 \pm 0.05$ & $14.20 \pm 0.03$ & $13.70 \pm 0.05$ & AAA & $\mathrm{N} / \mathrm{A}$ \\
\hline 165 & $203252.69+411019.4$ & $20325266+4110193$ & 0.74 & $14.05 \pm 0.03$ & $12.83 \pm 0.02$ & $12.40 \pm 0.02$ & AAA & 4.74 \\
\hline 166 & $203252.84+411955.0$ & $20325283+4119550$ & 0.27 & $13.23 \pm 0.02$ & $12.56 \pm 0.02$ & $12.23 \pm 0.02$ & AAA & 7.75 \\
\hline 167 & $203253.12+411559.0$ & $20325310+4115589$ & 0.50 & $12.93 \pm 0.02$ & $12.51 \pm 0.02$ & $12.32 \pm 0.02$ & AAA & 8.50 \\
\hline 168 & $203253.37+411927.7$ & $20325342+4119284$ & 0.79 & $15.26 \pm 0.05$ & $14.15 \pm 0.05$ & $13.71 \pm 0.05$ & AAA & 7.09 \\
\hline 169 & $203253.55+410714.1$ & - & - & - & - & - & - & - \\
\hline 170 & $203253.53+411545.3$ & $20325354+4115455$ & 0.12 & $16.27 \pm 0.11$ & $14.96 \pm 0.07$ & $14.62 \pm 0.11$ & AAB & 16.07 \\
\hline 171 & $203253.56+412019.4$ & $20325351+4120195$ & 1.01 & 16.36 & $15.32 \pm 0.10$ & $14.70 \pm 0.12$ & UAB & 7.54 \\
\hline 172 & $203253.77+411513.3$ & $20325377+4115134$ & 0.18 & $14.12 \pm 0.03$ & $12.96 \pm 0.03$ & $12.49 \pm 0.03$ & AAA & 8.20 \\
\hline 173 & $203253.88+411216.0$ & $20325385+4112151$ & 1.27 & 17.04 & $15.62 \pm 0.12$ & $57 \pm 0.10$ & UBA & 5.19 \\
\hline 174 & $203253.90+411536.7$ & $20325388+4$ & 0.59 & $13.37 \pm 0.02$ & $12.61 \pm 0.02$ & $12.32 \pm 0.02$ & AAA & N/A \\
\hline 175 & $203253.95+411702.7$ & $20325393+4117030$ & 0.35 & $16.13 \pm 0.08$ & $14.87 \pm 0.06$ & $14.24 \pm 0.07$ & AAA & 9.31 \\
\hline 176 & $203254.09+411726.5$ & $20325419+4117257$ & 1.69 & $16.82 \pm 0.15$ & $15.81 \pm 0.14$ & 15.60 & $\mathrm{CBU}$ & N/A \\
\hline 177 & $203254.17+411128.1$ & - & - & - & - & - & - & - \\
\hline 178 & $203254.22+410818.2$ & $20325412+4108185$ & 1.55 & $13.15 \pm 0.03$ & $12.06 \pm 0.02$ & $11.63 \pm 0.02$ & AAA & 5.95 \\
\hline 179 & $203254.19+411242.4$ & $20325421+4112426$ & 0.11 & $15.28 \pm 0.04$ & $13.96 \pm 0.03$ & $13.39 \pm 0.04$ & AAA & N/A \\
\hline 180 & $203254.21+411025.4$ & $20325422+4110256$ & 0.12 & 13.11 & $12.30 \pm 0.03$ & 12.02 & UAU & 9.21 \\
\hline 181 & $203254.28+411433.5$ & $20325431+4114341$ & 0.45 & 16.56 & $15.79 \pm 0.14$ & $15.11 \pm 0.18$ & UBC & 6.40 \\
\hline 182 & $203254.28+412333.2$ & $20325435+4123342$ & 1.12 & $15.55 \pm 0.06$ & $14.44 \pm 0.05$ & $14.03 \pm 0.07$ & AAA & 10.78 \\
\hline 183 & $203254.33+411458.5$ & $20325432+4114586$ & 0.27 & $14.88 \pm 0.04$ & $13.69 \pm 0.04$ & $13.22 \pm 0.04$ & AAA & 12.78 \\
\hline 184 & $203254.39+410640.9$ & $20325438+4106412$ & 0.34 & $14.54 \pm 0.03$ & $13.26 \pm 0.03$ & $12.83 \pm 0.03$ & AAA & 6.96 \\
\hline 185 & $203254.43+411550.0$ & $20325439+4115499$ & 0.73 & 14.43 & $14.88 \pm 0.09$ & $14.44 \pm 0.10$ & UAA & 2.57 \\
\hline 186 & $203254.56+410840.2$ & $20325469+4108396$ & 2.04 & $15.91 \pm 0.07$ & $14.15 \pm 0.05$ & $13.42 \pm 0.04$ & AAA & 1.25 \\
\hline 187 & $203254.55+411446.5$ & $20325457+4114468$ & 0.16 & $12.69 \pm 0.02$ & $11.79 \pm 0.02$ & $11.37 \pm 0.02$ & AAA & N/A \\
\hline 188 & $203254.61+411341.4$ & $20325461+4113416$ & 0.23 & $15.87 \pm 0.07$ & $14.52 \pm 0.05$ & $13.86 \pm 0.06$ & AAA & 9.95 \\
\hline 189 & $203254.60+411932.8$ & $20325464+4119321$ & 1.01 & $13.28 \pm 0.03$ & $12.54 \pm 0.02$ & $12.21 \pm 0.02$ & AAA & 7.03 \\
\hline 190 & $203254.79+411203.0$ & $20325480+4112034$ & 0.12 & $15.58 \pm 0.05$ & $14.10 \pm 0.03$ & $13.58 \pm 0.04$ & AAA & 4.91 \\
\hline 191 & $203254.89+411517.8$ & $20325486+4115180$ & 0.56 & $15.91 \pm 0.14$ & $14.60 \pm 0.11$ & 12.64 & $\mathrm{BBU}$ & 18.77 \\
\hline 192 & $203255.02+411038.8$ & $20325498+4110384$ & 0.96 & 16.50 & $15.71 \pm 0.12$ & 14.59 & UBU & N/A \\
\hline 193 & $203255.12+411314.8$ & $20325514+4113148$ & 0.24 & 15.35 & $13.95 \pm 0.04$ & $13.44 \pm 0.04$ & UAA & N/A \\
\hline 194 & $203255.15+411651.9$ & - & - & - & - & - & - & - \\
\hline 195 & $203255.27+410832.0$ & $20325525+4108321$ & 0.46 & $15.83 \pm 0.06$ & $14.49 \pm 0.04$ & $13.99 \pm 0.06$ & AAA & N/A \\
\hline
\end{tabular}


Table 2. continued.

\begin{tabular}{|c|c|c|c|c|c|c|c|c|}
\hline \multirow{2}{*}{$\begin{array}{l}\overline{N_{\mathrm{x}}} \\
\#\end{array}$} & \multicolumn{2}{|c|}{ X-ray - 2MASS counterpart } & \multirow{2}{*}{$\begin{array}{l}\text { Off. } \\
\left({ }^{\prime \prime}\right)\end{array}$} & \multicolumn{4}{|c|}{ 2MASS photometry } & \multirow{2}{*}{$\begin{array}{l}\begin{array}{l}A_{\mathrm{v}} \\
(\mathrm{mag})\end{array} \\
\text { (a) }\end{array}$} \\
\hline & CXOAC J+ & 2MASS J+ & & $J$ & $\bar{H}$ & $\bar{K}_{\mathrm{s}}$ & Ph. qual & \\
\hline 196 & $203255.26+411747.5$ & - & - & - & - & - & - & - \\
\hline 197 & $203255.33+411821.9$ & $20325536+4118216$ & 0.66 & 16.44 & $15.51 \pm 0.10$ & 14.63 & UAU & 8.83 \\
\hline 198 & $203255.34+411852.5$ & $20325536+4118529$ & 0.19 & $13.26 \pm 0.02$ & $12.25 \pm 0.02$ & $11.89 \pm 0.02$ & AAA & 0.21 \\
\hline 199 & $203255.67+411519.0$ & $20325570+4115194$ & 0.50 & $16.14 \pm 0.09$ & $14.89 \pm 0.06$ & $14.40 \pm 0.09$ & AAA & 6.31 \\
\hline 200 & $203255.78+411012.2$ & $20325574+4110120$ & 0.77 & $15.07 \pm 0.04$ & $13.82 \pm 0.04$ & $13.20 \pm 0.04$ & AAA & 6.34 \\
\hline 201 & $203255.72+412246.6$ & $20325572+4122475$ & 0.69 & $15.53 \pm 0.06$ & $14.51 \pm 0.04$ & $14.01 \pm 0.06$ & AAA & 3.78 \\
\hline 202 & $203255.86+412233.4$ & $20325597+4122343$ & 1.56 & $15.45 \pm 0.07$ & $14.28 \pm 0.06$ & $13.80 \pm 0.06$ & AAA & N/A \\
\hline 203 & $203256.28+411317.6$ & $20325632+4113180$ & 0.47 & $16.62 \pm 0.12$ & $15.15 \pm 0.07$ & $14.55 \pm 0.10$ & BAA & 5.55 \\
\hline 204 & $203256.35+411436.7$ & $20325633+4114366$ & 0.66 & $15.36 \pm 0.04$ & $14.15 \pm 0.04$ & $13.55 \pm 0.04$ & AAA & 11.00 \\
\hline 205 & $203256.40+411206.6$ & $20325647+4112075$ & 1.20 & $11.07 \pm 0.02$ & $10.43 \pm 0.01$ & $10.09 \pm 0.01$ & AAA & N/A \\
\hline 206 & $203256.49+411053.1$ & $20325648+4110531$ & 0.26 & $14.73 \pm 0.03$ & $13.32 \pm 0.03$ & $12.67 \pm 0.03$ & AAA & 8.75 \\
\hline 207 & $203256.55+411314.8$ & - & - & - & - & - & - & - \\
\hline 208 & $203256.61+411401.9$ & $20325661+4114017$ & 0.43 & $16.47 \pm 0.11$ & $15.09 \pm 0.08$ & $14.67 \pm 0.12$ & BAB & 9.30 \\
\hline 209 & $203256.66+412015.9$ & $20325669+4120161$ & 0.44 & $15.44 \pm 0.05$ & $14.21 \pm 0.04$ & $13.69 \pm 0.05$ & AAA & N/A \\
\hline 210 & $203256.71+411044.8$ & - & - & - & - & - & - & - \\
\hline 211 & $203256.69+412025.4$ & $20325666+4120255$ & 0.56 & $15.89 \pm 0.06$ & $14.52 \pm 0.05$ & $14.09 \pm$ & AAA & 2.88 \\
\hline 212 & $203256.74+411215.8$ & $20325677+4112162$ & 0.45 & $16.30 \pm 0.11$ & $14.94 \pm 0.08$ & $14.29 \pm 0.08$ & AAA & N/A \\
\hline 213 & $203256.85+411441.0$ & $20325683+4114412$ & 0.33 & $16.64 \pm 0.12$ & $15.19 \pm 0.08$ & $14.75 \pm 0.12$ & BAB & 8.42 \\
\hline 214 & $203257.07+410903.8$ & - & - & - & - & - & - & - \\
\hline 215 & $203257.06+411313.9$ & - & - & - & - & - & - & - \\
\hline 216 & $203257.17+412123.3$ & $20325721+4121260$ & 2.51 & $12.78 \pm 0.02$ & $11.55 \pm 0.02$ & $11.07 \pm 0.02$ & AAA & 5.84 \\
\hline 217 & $203257.25+411445.4$ & $20325729+4114461$ & 0.72 & $15.65 \pm 0.05$ & $14.48 \pm 0.04$ & $14.06 \pm 0.07$ & AAA & N/A \\
\hline 218 & $203257.32+412047.5$ & - & - & - & - & - & - & - \\
\hline 219 & $203257.36+411333.3$ & $20325736+4113334$ & 0.15 & $15.33 \pm 0.05$ & $14.00 \pm 0.04$ & $13.49 \pm 0.05$ & AAA & 8.25 \\
\hline 220 & $203257.56+411234.7$ & $20325758+4112352$ & 0.38 & $15.26 \pm 0.04$ & $13.97 \pm 0.04$ & $13.51 \pm 0.05$ & AAA & 0.25 \\
\hline 221 & $203257.73+411029.4$ & - & - & - & - & - & - & - \\
\hline 222 & $203257.80+410937.9$ & - & - & - & - & - & - & - \\
\hline 223 & $203257.82+411359.1$ & $20325781+4113593$ & 0.23 & $15.94 \pm 0.07$ & $14.61 \pm 0.05$ & $13.88 \pm 0.06$ & AAA & 6.13 \\
\hline 224 & $203257.91+411619.0$ & $20325792+4116190$ & 0.23 & $15.05 \pm 0.05$ & $13.93 \pm 0.05$ & 13.34 & AAU & N/A \\
\hline 225 & $203257.94+412156.9$ & - & - & - & - & - & - & - \\
\hline 226 & $203258.03+411058.1$ & $20325803+4110582$ & 0.13 & $15.14 \pm 0.04$ & $13.99 \pm 0.03$ & $13.48 \pm 0.04$ & AAA & 6.01 \\
\hline 227 & $203258.09+410713.6$ & $20325802+4107138$ & 1.21 & $17.00 \pm 0.17$ & $15.83 \pm 0.13$ & $15.07 \pm 0.15$ & $\mathrm{CBC}$ & 12.54 \\
\hline 228 & $203258.16+411735.0$ & $20325816+4117347$ & 0.53 & $2 \pm 0.14$ & $15.64 \pm 0.11$ & $14.34 \pm 0.09$ & $\mathrm{CBA}$ & 8.37 \\
\hline 229 & $203258.22+411250.6$ & $20325828+4112511$ & 0.83 & $17.00 \pm 0.18$ & $15.68 \pm 0.11$ & 15.12 & $\mathrm{CBU}$ & 6.93 \\
\hline 230 & $203258.27+411617.8$ & $20325825+4116177$ & 0.44 & $16.13 \pm 0.09$ & $14.86 \pm 0.06$ & 13.67 & AAU & 8.72 \\
\hline 231 & $203258.27+412147.8$ & $20325825+4121471$ & 0.96 & $15.90 \pm 0.07$ & $14.50 \pm 0.05$ & $14.12 \pm$ & AAA & 16.30 \\
\hline 232 & $203258.37+412000.9$ & - & - & - & - & - & - & - \\
\hline 233 & $203258.39+411543.2$ & - & - & - & - & - & - & - \\
\hline 234 & $203258.44+411105.4$ & - & - & - & - & - & - & - \\
\hline 235 & $203258.45+411403.9$ & $20325845+4114042$ & 0.26 & $15.82 \pm 0.07$ & $14.53 \pm 0.06$ & $13.99 \pm 0.07$ & AAA & 12.50 \\
\hline 236 & $203258.52+411234.8$ & $20325853+4112353$ & 0.37 & $16.38 \pm 0.11$ & $15.04 \pm 0.09$ & $14.53 \pm 0.10$ & BAA & N/A \\
\hline 237 & $203258.54+411525.2$ & $20325859+4115253$ & 0.56 & 16.53 & $15.23 \pm 0.08$ & $14.72 \pm 0.12$ & UAB & 17.45 \\
\hline 238 & $203258.73+411226.6$ & $20325874+4112266$ & 0.23 & $16.29 \pm 0.10$ & $14.81 \pm 0.07$ & $14.33 \pm 0.08$ & AAA & N/A \\
\hline 239 & $203258.74+411110.0$ & $20325883+4111095$ & 1.38 & $16.25 \pm 0.10$ & $14.60 \pm 0.08$ & $13.98 \pm 0.08$ & AAA & 5.08 \\
\hline 240 & $203258.93+411202.8$ & - & - & - & - & - & - & - \\
\hline 241 & $203258.93+411840.7$ & $20325896+4118410$ & 0.33 & 16.64 & $15.42 \pm 0.10$ & $14.46 \pm 0.09$ & UAA & 27.48 \\
\hline 242 & $203259.00+411051.8$ & $20325899+4110518$ & 0.36 & $13.06 \pm 0.02$ & $12.22 \pm 0.02$ & $11.78 \pm 0.02$ & AAA & 8.47 \\
\hline 243 & $203259.04+411758.7$ & $20325904+4$ & 0.11 & \pm 0.02 & $11.50 \pm 0.01$ & $11.26 \pm$ & AAA & 7.13 \\
\hline 244 & $203259.17+411937.4$ & $20325916+4119376$ & 0.22 & $10.55 \pm 0.02$ & $10.35 \pm 0.02$ & $10.30 \pm$ & AAA & N/A \\
\hline 245 & $203259.20+411229.5$ & - & - & - & - & - & - & - \\
\hline 246 & $203259.21+412049.0$ & $20325925+4120512$ & 2.10 & $15.75 \pm 0.06$ & $14.75 \pm 0.06$ & $14.06 \pm 0.07$ & AAA & N/A \\
\hline 247 & $203259.24+412137.4$ & $20325933+4121379$ & 1.23 & $11.40 \pm 0.02$ & $10.70 \pm 0.01$ & $10.23 \pm 0.01$ & AAA & N/A \\
\hline 248 & $203259.24+412238.4$ & $20325916+4122386$ & 1.32 & $15.70 \pm 0.06$ & $14.35 \pm 0.05$ & $13.96 \pm 0.06$ & AAA & 7.54 \\
\hline 249 & $203259.31+411037.4$ & $20325932+4110376$ & 0.12 & $16.20 \pm 0.09$ & $14.88 \pm 0.06$ & $14.32 \pm 0.08$ & AAA & 0.56 \\
\hline 250 & $203259.38+411223.5$ & - & - & - & - & - & - & - \\
\hline 251 & $203259.45+410941.4$ & $20325954+4109434$ & 2.14 & 16.84 & 15.31 & $14.85 \pm 0.14$ & UUB & 13.89 \\
\hline 252 & $203259.57+411055.1$ & $20325955+4110560$ & 0.78 & 16.52 & $15.46 \pm 0.12$ & 14.79 & UBU & 6.02 \\
\hline 253 & $203259.63+411514.6$ & $20325964+4115146$ & 0.17 & $9.05 \pm 0.02$ & $8.45 \pm 0.01$ & $8.14 \pm 0.01$ & AAA & 8.97 \\
\hline 254 & $203259.63+411941.0$ & $20325959+4119409$ & 0.77 & $15.16 \pm 0.17$ & $13.90 \pm 0.09$ & $13.48 \pm 0.06$ & CAA & N/A \\
\hline 255 & $203259.75+411300.0$ & $20325972+4112599$ & 0.65 & $14.92 \pm 0.04$ & $13.87 \pm 0.03$ & $13.32 \pm 0.04$ & AAA & 7.58 \\
\hline 256 & $203259.75+411419.4$ & $20325976+4114196$ & 0.01 & $10.78 \pm 0.02$ & $10.18 \pm 0.02$ & $9.87 \pm 0.02$ & AAA & 0.79 \\
\hline 257 & $203259.77+411345.4$ & - & - & - & - & - & - & - \\
\hline 258 & $203259.85+412036.5$ & - & - & - & - & - & - & - \\
\hline 259 & $203259.94+411027.9$ & $20325995+4110280$ & 0.16 & $16.79 \pm 0.14$ & $15.24 \pm 0.08$ & $14.71 \pm 0.11$ & $\mathrm{CAB}$ & 5.64 \\
\hline 260 & $203259.98+410848.6$ & $20325987+4108488$ & 1.76 & 15.22 & $14.32 \pm 0.06$ & $13.88 \pm 0.06$ & UAA & N/A \\
\hline
\end{tabular}


Table 2. continued.

\begin{tabular}{|c|c|c|c|c|c|c|c|c|}
\hline \multirow{2}{*}{$\begin{array}{l}N_{\mathrm{x}} \\
\#\end{array}$} & \multicolumn{2}{|c|}{ X-ray - 2MASS counterpart } & \multirow{2}{*}{$\begin{array}{l}\text { Off. } \\
\left({ }^{\prime \prime}\right)\end{array}$} & \multicolumn{4}{|c|}{ 2MASS photometry } & \multirow{2}{*}{$\begin{array}{l}\begin{array}{l}A_{\mathrm{v}} \\
(\mathrm{mag})\end{array} \\
\text { (a) }\end{array}$} \\
\hline & CXOAC J+ & 2MASS J+ & & $J$ & $H$ & $K_{\mathrm{s}}$ & Ph. qual & \\
\hline 261 & $203300.01+411107.2$ & $20330000+4111073$ & 0.35 & $15.72 \pm 0.06$ & $14.98 \pm 0.07$ & $14.76 \pm 0.13$ & AAB & N/A \\
\hline 262 & $203300.16+411217.6$ & $20330015+4112178$ & 0.23 & $16.40 \pm 0.11$ & $15.06 \pm 0.09$ & $14.49 \pm 0.09$ & BAA & 12.50 \\
\hline 263 & $203300.29+411656.6$ & - & - & - & - & - & - & - \\
\hline 264 & $203300.38+411730.6$ & $20330041+4117308$ & 0.44 & $16.32 \pm 0.10$ & $14.89 \pm 0.07$ & $14.34 \pm 0.09$ & AAA & 8.49 \\
\hline 265 & $203300.38+411651.7$ & $20330038+4116520$ & 0.22 & $16.46 \pm 0.12$ & $15.20 \pm 0.08$ & $14.58 \pm 0.11$ & BAB & 6.64 \\
\hline 266 & $203300.42+411124.4$ & $20330035+4111252$ & 1.34 & $15.06 \pm 0.04$ & $13.49 \pm 0.03$ & $12.65 \pm 0.03$ & AAA & 4.00 \\
\hline 267 & $203300.41+412030.8$ & - & - & - & - & - & - & - \\
\hline 268 & $203300.46+411147.5$ & $20330050+4111476$ & 0.46 & 13.33 & $12.29 \pm 0.02$ & $11.90 \pm 0.02$ & UAA & 1.85 \\
\hline 269 & $203300.52+411550.1$ & - & - & - & - & - & - & - \\
\hline 270 & $203300.52+412214.0$ & $20330032+4122141$ & 3.08 & $15.45 \pm 0.04$ & $14.22 \pm 0.04$ & $13.74 \pm 0.05$ & AAA & N/A \\
\hline 271 & $203300.59+411859.3$ & $20330058+4118594$ & 0.36 & $15.87 \pm 0.08$ & 14.39 & 13.86 & AUU & 8.67 \\
\hline 272 & $203300.73+410929.4$ & $20330070+4109299$ & 0.65 & $12.21 \pm 0.02$ & $11.48 \pm 0.01$ & $11.11 \pm 0.01$ & AAA & 14.31 \\
\hline 273 & $203300.77+411627.1$ & $20330078+4116275$ & 0.10 & $15.54 \pm 0.05$ & $14.15 \pm 0.04$ & $13.64 \pm 0.05$ & AAA & 23.09 \\
\hline 274 & $203300.77+412032.1$ & - & - & - & - & - & - & - \\
\hline 275 & $203300.84+412133.8$ & $20330091+4121339$ & 0.98 & 16.09 & $14.92 \pm 0.07$ & 14.59 & UAU & 1.87 \\
\hline 276 & $203300.88+411115.6$ & $20330088+4111157$ & 0.29 & $14.65 \pm 0.04$ & $13.20 \pm 0.04$ & $12.70 \pm 0.03$ & AAA & 9.81 \\
\hline 277 & $203300.90+411736.7$ & $20330091+4117367$ & 0.28 & $15.91 \pm 0.06$ & $14.62 \pm 0.05$ & $14.02 \pm 0.06$ & AAA & N/A \\
\hline 278 & $203300.95+411423.8$ & $20330097+4114241$ & 0.13 & $15.27 \pm 0.05$ & $13.92 \pm 0.03$ & $13.50 \pm 0.04$ & AAA & 9.89 \\
\hline 279 & $203300.95+412119.9$ & $20330103+4121201$ & 1.10 & $16.08 \pm 0.08$ & $15.13 \pm 0.07$ & $14.55 \pm 0.10$ & AAA & 8.77 \\
\hline 280 & $203301.09+411755.7$ & $20330106+4117564$ & 0.65 & $13.54 \pm 0.02$ & $12.53 \pm 0.02$ & $12.09 \pm 0.02$ & AAA & 7.62 \\
\hline 281 & $203301.15+411321.8$ & $20330116+4113218$ & 0.20 & 16.97 & $15.84 \pm 0.13$ & $15.06 \pm 0.16$ & $\mathrm{UBC}$ & 6.10 \\
\hline 282 & $203301.16+411152.7$ & $20330118+4111525$ & 0.50 & 16.78 & $15.68 \pm 0.12$ & 14.78 & UBU & N/A \\
\hline 283 & $203301.20+410918.2$ & $20330119+4109182$ & 0.30 & $13.96 \pm 0.03$ & $12.79 \pm 0.03$ & $12.30 \pm 0.03$ & AAA & 5.71 \\
\hline 284 & $203301.21+410858.0$ & $20330124+4108580$ & 0.27 & $15.15 \pm 0.04$ & $13.82 \pm 0.04$ & $13.31 \pm 0.04$ & AAA & 7.81 \\
\hline 285 & $203301.25+411459.5$ & $20330127+4114596$ & 0.22 & 14.91 & 13.61 & $13.62 \pm 0.05$ & UUA & 7.94 \\
\hline 286 & $203301.26+411256.8$ & - & - & - & - & - & - & - \\
\hline 287 & $203301.35+411055.5$ & $20330133+4110562$ & 0.62 & 16.87 & $15.50 \pm 0.11$ & $15.34 \pm 0.21$ & $\mathrm{UBC}$ & 5.94 \\
\hline 288 & $203301.35+411223.6$ & - & - & - & - & - & - & - \\
\hline 289 & $203301.35+411331.2$ & $20330133+4113316$ & 0.46 & 16.44 & $15.72 \pm 0.14$ & 15.17 & UBU & 9.69 \\
\hline 290 & $203301.38+412158.5$ & $20330156+4121579$ & 2.77 & $15.35 \pm 0.05$ & $14.14 \pm 0.05$ & $13.63 \pm 0.05$ & AAA & 8.11 \\
\hline 291 & $203301.43+411200.2$ & $20330142+4112000$ & 0.51 & $16.29 \pm 0.10$ & $15.02 \pm 0.07$ & $14.38 \pm 0.08$ & AAA & N/A \\
\hline 292 & $203301.49+411211.5$ & $20330150+4112115$ & 0.10 & $16.69 \pm 0.14$ & $15.42 \pm 0.11$ & 14.42 & BAU & 5.99 \\
\hline 293 & $203301.49+411211.6$ & $20330150+4112116$ & 0.19 & $16.79 \pm 0.11$ & $15.32 \pm 0.12$ & 14.49 & BAU & N/A \\
\hline 294 & $203301.58+412302.9$ & - & - & - & - & - & - & - \\
\hline 295 & $203301.67+411248.2$ & $20330168+4112486$ & 0.10 & $15.03 \pm 0.05$ & $13.62 \pm 0.04$ & $13.22 \pm 0.04$ & AAA & 9.08 \\
\hline 296 & $203301.66+411959.0$ & $20330155+4119591$ & 1.77 & $16.39 \pm 0.10$ & $15.11 \pm 0.07$ & $14.47 \pm 0.09$ & BAA & N/A \\
\hline 297 & $203301.68+411751.4$ & $20330169+4117513$ & 0.24 & $14.71 \pm 0.03$ & $13.58 \pm 0.03$ & $13.11 \pm 0.03$ & AAA & 10.36 \\
\hline 298 & $203301.72+411147.0$ & $20330170+4111475$ & 0.43 & $13.41 \pm 0.02$ & $12.61 \pm 0.02$ & $12.22 \pm 0.02$ & AAA & 14.35 \\
\hline 299 & $203301.72+411500.5$ & $20330173+4115007$ & 0.15 & $14.26 \pm 0.03$ & $12.98 \pm 0.05$ & $12.48 \pm 0.03$ & AAA & 7.54 \\
\hline 300 & $203301.77+411110.5$ & $20330176+4111107$ & 0.11 & $16.42 \pm 0.12$ & 14.92 & 14.48 & BUU & N/A \\
\hline 301 & $203301.80+411103.9$ & $20330182+4111037$ & 0.36 & $16.25 \pm 0.10$ & $14.76 \pm 0.06$ & $14.36 \pm 0.09$ & AAA & N/A \\
\hline 302 & $203301.83+411317.3$ & $20330182+4113174$ & 0.24 & $16.19 \pm 0.09$ & $14.97 \pm 0.06$ & $14.39 \pm 0.09$ & AAA & 10.17 \\
\hline 303 & $203301.94+411059.0$ & $20330198+4110593$ & 0.55 & $15.05 \pm 0.04$ & $13.81 \pm 0.04$ & $13.26 \pm 0.04$ & AAA & 7.64 \\
\hline 304 & $203301.93+411739.3$ & $20330193+4117400$ & 0.50 & $14.99 \pm 0.09$ & $13.84 \pm 0.08$ & $13.43 \pm 0.06$ & AAA & N/A \\
\hline 305 & $203301.97+410644.4$ & $20330191+4106457$ & 1.44 & $16.80 \pm 0.15$ & $15.32 \pm 0.08$ & $14.75 \pm 0.12$ & $\mathrm{CAB}$ & 7.43 \\
\hline 306 & $203301.95+411946.8$ & $20330197+4119466$ & 0.52 & $15.44 \pm 0.05$ & $14.21 \pm 0.05$ & $13.74 \pm 0.06$ & AAA & 5.07 \\
\hline 307 & $203302.18+411848.8$ & $20330224+4118489$ & 0.66 & $13.29 \pm 0.02$ & $12.60 \pm 0.02$ & $12.22 \pm 0.02$ & AAA & 8.17 \\
\hline 308 & $203302.26+411533.4$ & $20330226+4$ & 0.11 & 14 & $13.23 \pm 0.04$ & $2 \pm 0.03$ & AAA & 7.54 \\
\hline 309 & $203302.31+411324.2$ & $20330231+4113245$ & 0.14 & $15.27 \pm 0.06$ & $13.84 \pm 0.09$ & $13.36 \pm 0.04$ & AAA & 7.31 \\
\hline 310 & $203302.35+411828.1$ & $20330231+4118285$ & 0.79 & $12.04 \pm 0.02$ & $11.26 \pm 0.02$ & $10.88 \pm 0.01$ & AAA & N/A \\
\hline 311 & $203302.40+411738.4$ & $20330249+4117393$ & 1.44 & $14.74 \pm 0.14$ & 11.42 & 10.84 & BUU & 1.50 \\
\hline 312 & $203302.63+411355.7$ & $20330259+4113563$ & 0.85 & $16.83 \pm 0.14$ & $15.44 \pm 0.10$ & $14.84 \pm 0.13$ & $\mathrm{CAB}$ & 3.20 \\
\hline 313 & $203302.87+410705.9$ & $20330285+4107068$ & 0.80 & $16.18 \pm 0.09$ & $14.89 \pm 0.07$ & $14.30 \pm 0.08$ & AAA & N/A \\
\hline 314 & $203302.87+411514.0$ & - & - & - & - & - & - & - \\
\hline 315 & $203302.90+411742.9$ & $20330292+4117431$ & 0.12 & $8.72 \pm 0.02$ & $8.16 \pm 0.01$ & $7.87 \pm 0.01$ & AAA & N/A \\
\hline 316 & $203303.03+411329.2$ & - & - & - & - & - & - & - \\
\hline 317 & $203303.03+411740.1$ & - & - & - & - & - & - & - \\
\hline 318 & $203303.07+411243.4$ & $20330308+4112435$ & 0.12 & $14.83 \pm 0.04$ & $13.54 \pm 0.03$ & $12.94 \pm 0.03$ & AAA & N/A \\
\hline 319 & $203303.22+411629.4$ & $20330322+4116297$ & 0.08 & $15.77 \pm 0.06$ & $14.84 \pm 0.06$ & $14.29 \pm 0.08$ & AAA & 21.26 \\
\hline 320 & $203303.53+411253.6$ & $20330356+4112539$ & 0.34 & $15.63 \pm 0.06$ & $14.00 \pm 0.04$ & $13.26 \pm 0.04$ & AAA & 8.97 \\
\hline 321 & $203303.64+411236.6$ & - & - & - & - & - & - & - \\
\hline 322 & $203303.66+411334.2$ & - & - & - & - & - & - & - \\
\hline 323 & $203303.66+411218.2$ & $20330368+4112188$ & 0.42 & $15.87 \pm 0.10$ & $14.48 \pm 0.06$ & $13.87 \pm 0.06$ & AAA & 6.53 \\
\hline 324 & $203303.88+411736.2$ & $20330390+4117367$ & 0.26 & $13.05 \pm 0.02$ & $12.45 \pm 0.02$ & $12.10 \pm 0.02$ & AAA & 5.76 \\
\hline 325 & $203304.06+411124.5$ & $20330410+4111250$ & 0.62 & $16.13 \pm 0.09$ & $14.79 \pm 0.08$ & $14.19 \pm 0.08$ & AAA & N/A \\
\hline
\end{tabular}


Table 2. continued.

\begin{tabular}{|c|c|c|c|c|c|c|c|c|}
\hline \multirow{2}{*}{$\begin{array}{l}N_{\mathrm{x}} \\
\#\end{array}$} & \multicolumn{2}{|c|}{ X-ray - 2MASS counterpart } & \multirow{2}{*}{$\begin{array}{l}\text { Off. } \\
\left({ }^{\prime \prime}\right)\end{array}$} & \multicolumn{4}{|c|}{ 2MASS photometry } & \multirow{2}{*}{$\begin{array}{l}\begin{array}{l}A_{\mathrm{v}} \\
(\mathrm{mag})\end{array} \\
\text { (a) }\end{array}$} \\
\hline & CXOAC J+ & 2MASS J+ & & $J$ & $H$ & $K_{\mathrm{s}}$ & Ph.qual & \\
\hline 326 & $203304.10+410755.3$ & $20330408+4107542$ & 1.40 & $14.45 \pm 0.05$ & $13.26 \pm 0.08$ & $12.76 \pm 0.03$ & AAA & 9.16 \\
\hline 327 & $203304.16+410651.5$ & $20330423+4106514$ & 1.14 & $13.94 \pm 0.02$ & $12.74 \pm 0.02$ & $12.29 \pm 0.02$ & AAA & N/A \\
\hline 328 & $203304.22+411411.7$ & - & - & - & - & - & - & - \\
\hline 329 & $203304.26+410723.1$ & $20330435+4107237$ & 1.38 & $15.80 \pm 0.07$ & $14.42 \pm 0.04$ & $14.05 \pm 0.07$ & AAA & N/A \\
\hline 330 & $203304.24+411254.6$ & $20330425+4112548$ & 0.04 & $15.12 \pm 0.04$ & $13.81 \pm 0.03$ & $13.46 \pm 0.04$ & AAA & N/A \\
\hline 331 & $203304.28+411235.2$ & $20330428+4112354$ & 0.03 & 16.94 & $15.85 \pm 0.15$ & $14.98 \pm 0.15$ & UBB & 23.62 \\
\hline 332 & $203304.30+411613.4$ & $20330427+4116138$ & 0.56 & $15.75 \pm 0.07$ & $14.37 \pm 0.06$ & $13.71 \pm 0.06$ & AAA & 7.08 \\
\hline 333 & $203304.32+411420.4$ & $20330433+4114209$ & 0.27 & 15.87 & $14.37 \pm 0.04$ & 13.91 & UAU & 37.03 \\
\hline 334 & $203304.38+411405.3$ & $20330437+4114056$ & 0.33 & $16.11 \pm 0.08$ & $14.56 \pm 0.04$ & $14.04 \pm 0.06$ & AAA & 12.13 \\
\hline 335 & $203304.42+411830.8$ & $20330442+4118311$ & 0.13 & $13.82 \pm 0.02$ & $12.84 \pm 0.02$ & $12.41 \pm 0.02$ & AAA & N/A \\
\hline 336 & $203304.48+411128.1$ & $20330447+4111282$ & 0.25 & $16.44 \pm 0.12$ & $15.31 \pm 0.09$ & $14.98 \pm 0.15$ & BAB & N/A \\
\hline 337 & $203304.49+411157.6$ & $20330447+4111580$ & 0.48 & $16.22 \pm 0.10$ & $14.90 \pm 0.07$ & $14.48 \pm 0.10$ & AAA & 4.79 \\
\hline 338 & $203304.57+411612.9$ & - & - & - & - & - & - & - \\
\hline 339 & $203304.67+411205.9$ & $20330466+4112058$ & 0.48 & $16.00 \pm 0.08$ & $14.68 \pm 0.05$ & $13.98 \pm 0.06$ & AAA & 8.08 \\
\hline 340 & $203304.70+411506.2$ & $20330475+4115064$ & 0.66 & $16.31 \pm 0.10$ & $14.95 \pm 0.07$ & $14.36 \pm 0.09$ & AAA & 9.49 \\
\hline 341 & $203304.75+411312.4$ & $20330476+4113130$ & 0.39 & $15.79 \pm 0.09$ & $14.35 \pm 0.09$ & $13.91 \pm 0.08$ & AAA & 5.47 \\
\hline 342 & $203304.75+411650.4$ & - & - & - & - & - & - & - \\
\hline 343 & $203304.78+410857.2$ & - & - & - & - & - & - & - \\
\hline 344 & $203304.86+411238.7$ & $20330487+4112389$ & 0.06 & 15.52 & $14.23 \pm 0.08$ & $13.77 \pm 0.06$ & UAA & 11.20 \\
\hline 345 & $203304.95+411129.5$ & $20330495+4111291$ & 0.55 & $16.27 \pm 0.09$ & $14.81 \pm 0.07$ & $14.38 \pm 0.09$ & AAA & 3.32 \\
\hline 346 & $203305.01+411256.2$ & $20330500+4112566$ & 0.37 & $16.66 \pm 0.17$ & $15.03 \pm 0.07$ & $14.50 \pm 0.09$ & CAA & 10.59 \\
\hline 347 & $203305.03+411421.5$ & $20330505+4114219$ & 0.17 & $15.61 \pm 0.06$ & $14.08 \pm 0.03$ & $13.33 \pm 0.05$ & AAA & 7.86 \\
\hline 348 & $203305.03+411831.4$ & $20330502+4118317$ & 0.24 & $15.95 \pm 0.08$ & $14.66 \pm 0.06$ & $14.02 \pm 0.07$ & AAA & 5.06 \\
\hline 349 & $203305.04+411146.6$ & - & - & - & - & - & - & - \\
\hline 350 & $203305.03+411421.4$ & $20330505+4114219$ & 0.26 & $15.61 \pm 0.06$ & $14.08 \pm 0.03$ & $13.33 \pm 0.05$ & AAA & N/A \\
\hline 351 & $203305.05+411144.8$ & $20330503+4111452$ & 0.38 & $15.20 \pm 0.06$ & $13.74 \pm 0.05$ & $13.19 \pm 0.05$ & AAA & N/A \\
\hline 352 & $203305.17+411233.2$ & $20330519+4112336$ & 0.25 & $15.82 \pm 0.07$ & $14.25 \pm 0.05$ & $13.84 \pm 0.06$ & AAA & 8.31 \\
\hline 353 & $203305.20+411335.6$ & $20330519+4113360$ & 0.36 & $14.88 \pm 0.12$ & $13.90 \pm 0.09$ & $13.34 \pm 0.07$ & EAA & 6.78 \\
\hline 354 & $203305.25+411604.5$ & $20330525+4116047$ & 0.05 & $14.62 \pm 0.03$ & $13.86 \pm 0.03$ & $13.55 \pm 0.04$ & AAA & 0.40 \\
\hline 355 & $203305.26+412138.7$ & $20330526+4121391$ & 0.22 & $14.32 \pm 0.03$ & $13.15 \pm 0.03$ & $12.54 \pm 0.02$ & AAA & N/A \\
\hline 356 & $203305.28+411258.6$ & $20330528+4112591$ & 0.29 & $15.58 \pm 0.08$ & $14.23 \pm 0.10$ & $13.75 \pm 0.07$ & AAA & N/A \\
\hline 357 & $203305.30+411303.6$ & $20330529+4113038$ & 0.24 & $13.29 \pm 0.03$ & $12.56 \pm 0.03$ & $12.24 \pm 0.03$ & AAA & 7.98 \\
\hline 358 & $203305.36+411311.8$ & - & - & - & - & - & - & - \\
\hline 359 & $203305.41+411153.6$ & $20330541+4111538$ & 0.01 & 15.23 & $13.87 \pm 0.05$ & $13.37 \pm 0.05$ & UAA & 2.48 \\
\hline 360 & $203305.47+411610.5$ & $20330549+4116109$ & 0.27 & $16.29 \pm 0.10$ & $15.24 \pm 0.08$ & $14.55 \pm 0.11$ & AAA & N/A \\
\hline 361 & $203305.59+411348.2$ & $20330561+4113486$ & 0.15 & $14.45 \pm 0.02$ & $13.17 \pm 0.02$ & $12.75 \pm 0.03$ & AAA & 3.61 \\
\hline 362 & $203305.72+411136.7$ & - & - & - & - & - & - & - \\
\hline 363 & $203305.79+411634.7$ & $20330579+4116352$ & 0.28 & $16.62 \pm 0.13$ & $13.96 \pm 0.03$ & $12.30 \pm 0.02$ & BAA & 4.88 \\
\hline 364 & $203305.89+411905.0$ & $20330587+4119048$ & 0.54 & 16.85 & $15.60 \pm 0.12$ & 15.44 & UBU & N/A \\
\hline 365 & $203305.92+412057.5$ & $20330594+4120573$ & 0.42 & $16.10 \pm 0.09$ & $14.79 \pm 0.06$ & $14.40 \pm 0.09$ & AAA & N/A \\
\hline 366 & $203306.07+411256.7$ & - & - & - & - & - & - & - \\
\hline 367 & $203306.13+411012.6$ & $20330615+4110140$ & 1.26 & 15.82 & $15.37 \pm 0.09$ & 13.94 & UAU & N/A \\
\hline 368 & $203306.19+411453.8$ & - & - & - & - & - & - & - \\
\hline 369 & $203306.21+411715.3$ & $20330616+4117158$ & 0.82 & 16.19 & 14.78 & $14.59 \pm 0.12$ & UUB & N/A \\
\hline 370 & $203306.20+412242.9$ & $20330611+4$ & 1.46 & $16.03 \pm 0.09$ & $14.90 \pm 0.07$ & $14.19 \pm 0.08$ & AAA & 6.96 \\
\hline 371 & $203306.24+410948.3$ & $20330621+4109495$ & 1.10 & $15.16 \pm 0.05$ & $13.80 \pm 0.04$ & $13.26 \pm 0.04$ & AAA & 1.12 \\
\hline 372 & $203306.26+411235.1$ & $20330626+4112354$ & 0.17 & $14.19 \pm 0.02$ & $12.94 \pm 0.02$ & $12.44 \pm 0.02$ & AAA & 6.78 \\
\hline 373 & $203306.27+411100.4$ & - & - & - & - & - & - & - \\
\hline 374 & $203306.27+412019.0$ & $20330629+4120188$ & 0.44 & $14.81 \pm 0.03$ & $13.61 \pm 0.02$ & $13.10 \pm 0.03$ & AAA & 3.16 \\
\hline 375 & $203306.32+412215.2$ & $20330622+4122138$ & 2.32 & $16.15 \pm 0.10$ & $15.02 \pm 0.08$ & $14.45 \pm 0.10$ & AAA & 6.82 \\
\hline 376 & $203306.35+411353.1$ & $20330635+4113536$ & 0.30 & $16.03 \pm 0.08$ & $14.75 \pm 0.07$ & $14.25 \pm 0.09$ & AAA & 4.50 \\
\hline 377 & $203306.38+411330.7$ & $20330641+4113310$ & 0.22 & $14.77 \pm 0.04$ & $13.41 \pm 0.04$ & $13.01 \pm 0.04$ & AAA & 2.20 \\
\hline 378 & $203306.47+411206.8$ & $20330646+4112071$ & 0.23 & $13.97 \pm 0.03$ & $12.77 \pm 0.04$ & $12.30 \pm 0.03$ & AAA & 10.37 \\
\hline 379 & $203306.61+412158.3$ & - & - & - & - & - & - & - \\
\hline 380 & $203306.64+411817.7$ & - & - & - & - & - & - & - \\
\hline 381 & $203306.71+411200.0$ & $20330671+4112002$ & 0.12 & 14.50 & $13.14 \pm 0.02$ & 12.72 & UAU & 7.02 \\
\hline 382 & $203306.73+411527.5$ & - & - & - & - & - & - & - \\
\hline 383 & $203306.79+411727.8$ & $20330681+4117283$ & 0.25 & $15.61 \pm 0.06$ & $14.30 \pm 0.04$ & $13.89 \pm 0.07$ & AAA & N/A \\
\hline 384 & $203306.84+411224.3$ & $20330684+4112245$ & 0.11 & $15.99 \pm 0.08$ & $14.47 \pm 0.05$ & $13.90 \pm 0.06$ & AAA & N/A \\
\hline 385 & $203306.85+411503.4$ & - & - & - & - & - & - & - \\
\hline 386 & $203306.91+411647.2$ & - & - & - & - & - & - & - \\
\hline 387 & $203306.93+411858.9$ & $20330697+4118592$ & 0.44 & $15.06 \pm 0.05$ & $13.84 \pm 0.04$ & $13.42 \pm 0.05$ & AAA & 5.49 \\
\hline 388 & $203307.00+411649.6$ & $20330702+4116499$ & 0.11 & $14.84 \pm 0.03$ & $13.48 \pm 0.02$ & $12.80 \pm 0.03$ & AAA & $\mathrm{N} / \mathrm{A}$ \\
\hline 389 & $203307.06+411300.1$ & $20330711+4113002$ & 0.56 & $15.30 \pm 0.06$ & $13.94 \pm 0.05$ & $13.41 \pm 0.05$ & AAA & 16.66 \\
\hline 390 & $203307.07+411604.3$ & - & - & - & - & - & - & - \\
\hline
\end{tabular}


Table 2. continued.

\begin{tabular}{|c|c|c|c|c|c|c|c|c|}
\hline \multirow{2}{*}{$\begin{array}{l}N_{\mathrm{x}} \\
\#\end{array}$} & \multicolumn{2}{|c|}{ X-ray - 2MASS counterpart } & \multirow{2}{*}{$\begin{array}{l}\text { Off. } \\
\left({ }^{\prime \prime}\right)\end{array}$} & \multicolumn{4}{|c|}{ 2MASS photometry } & \multirow{2}{*}{$\begin{array}{l}\begin{array}{l}A_{\mathrm{v}} \\
(\mathrm{mag})\end{array} \\
\text { (a) }\end{array}$} \\
\hline & CXOAC J+ & 2MASS J+ & & $J$ & $H$ & $K_{\mathrm{s}}$ & Ph.qual & \\
\hline 391 & $203307.13+411625.7$ & $20330713+4116263$ & 0.38 & 17.16 & $15.55 \pm 0.10$ & $14.93 \pm 0.15$ & UBB & 4.92 \\
\hline 392 & $203307.14+411141.2$ & - & - & - & - & - & - & - \\
\hline 393 & $203307.14+412006.9$ & $20330712+4120072$ & 0.45 & $14.24 \pm 0.02$ & $13.03 \pm 0.02$ & $12.52 \pm 0.02$ & AAA & N/A \\
\hline 394 & $203307.15+411318.7$ & - & - & - & - & - & - & - \\
\hline 395 & $203307.16+411730.4$ & - & - & - & - & - & - & - \\
\hline 396 & $203307.17+411520.2$ & $20330718+4115206$ & 0.26 & $16.15 \pm 0.09$ & $14.83 \pm 0.06$ & $14.18 \pm 0.08$ & AAA & 4.51 \\
\hline 397 & $203307.25+411000.4$ & $20330736+4110014$ & 1.60 & 16.48 & $15.49 \pm 0.10$ & $14.86 \pm 0.13$ & UAB & N/A \\
\hline 398 & $203307.32+411214.6$ & $20330733+4112148$ & 0.05 & $14.67 \pm 0.11$ & $13.28 \pm 0.03$ & $12.74 \pm 0.03$ & BAA & 6.72 \\
\hline 399 & $203307.34+411341.5$ & - & - & - & - & - & - & - \\
\hline 400 & $203307.36+411800.0$ & $20330737+4118000$ & 0.23 & $16.32 \pm 0.10$ & $15.06 \pm 0.08$ & $14.57 \pm 0.11$ & AAA & 4.87 \\
\hline 401 & $203307.40+411151.3$ & $20330739+4111505$ & 1.09 & $16.02 \pm 0.08$ & $14.40 \pm 0.06$ & $13.75 \pm 0.06$ & AAA & 6.35 \\
\hline 402 & $203307.45+411226.4$ & $20330746+4112266$ & 0.06 & $13.16 \pm 0.02$ & $12.02 \pm 0.02$ & $11.50 \pm 0.02$ & AAA & N/A \\
\hline 403 & $203307.47+411254.5$ & - & - & - & - & - & - & - \\
\hline 404 & $203307.50+412032.3$ & $20330747+4120324$ & 0.57 & 17.38 & $15.64 \pm 0.12$ & 15.31 & UBU & 7.39 \\
\hline 405 & $203307.55+411821.2$ & $20330751+4118214$ & 0.66 & $16.18 \pm 0.09$ & $14.85 \pm 0.05$ & $14.42 \pm 0.09$ & AAA & 11.82 \\
\hline 406 & $203307.57+411947.6$ & $20330758+4119478$ & 0.01 & $14.18 \pm 0.03$ & $13.12 \pm 0.03$ & $12.68 \pm 0.02$ & AAA & 5.51 \\
\hline 407 & $203307.59+411300.7$ & $20330759+4113010$ & 0.04 & $14.05 \pm 0.06$ & 12.60 & $12.39 \pm 0.05$ & AUA & N/A \\
\hline 408 & $203307.73+410828.5$ & $20330768+4108291$ & 0.97 & $13.75 \pm 0.04$ & $12.73 \pm 0.03$ & $12.31 \pm 0.03$ & AAA & 4.66 \\
\hline 409 & $203307.83+411243.0$ & $20330783+4112435$ & 0.23 & $16.27 \pm 0.12$ & $15.09 \pm 0.09$ & $14.68 \pm 0.15$ & BAB & 4.81 \\
\hline 410 & $203307.86+411216.3$ & $20330786+4112161$ & 0.45 & $12.84 \pm 0.03$ & $11.91 \pm 0.05$ & $11.44 \pm 0.04$ & AAA & N/A \\
\hline 411 & $203307.91+411254.3$ & - & - & - & - & - & - & - \\
\hline 412 & $203307.91+411606.9$ & $20330791+4116074$ & 0.33 & 16.68 & $15.46 \pm 0.13$ & 14.79 & UBU & N/A \\
\hline 413 & $203307.97+411257.7$ & $20330792+4112571$ & 1.12 & $15.41 \pm 0.08$ & 13.03 & $13.48 \pm 0.07$ & AUA & 7.59 \\
\hline 414 & $203307.98+411300.7$ & $20330798+4113007$ & 0.18 & $15.02 \pm 0.07$ & 12.73 & $13.23 \pm 0.05$ & AUA & 9.77 \\
\hline 415 & $203307.99+411659.4$ & $20330801+4116597$ & 0.11 & $15.81 \pm 0.07$ & $14.55 \pm 0.05$ & $14.05 \pm 0.07$ & AAA & 3.74 \\
\hline 416 & $203308.02+411801.5$ & $20330806+4118016$ & 0.48 & $13.93 \pm 0.02$ & $13.28 \pm 0.03$ & $12.99 \pm 0.03$ & AAA & N/A \\
\hline 417 & $203308.03+411436.9$ & - & - & - & - & - & - & - \\
\hline 418 & $203308.04+411642.9$ & - & - & - & - & - & - & - \\
\hline 419 & $203308.05+412217.4$ & $20330808+4122180$ & 0.44 & $15.30 \pm 0.05$ & $14.11 \pm 0.04$ & $13.63 \pm 0.05$ & AAA & N/A \\
\hline 420 & $203308.09+411109.6$ & $20330808+4111091$ & 0.66 & 13.37 & $12.52 \pm 0.04$ & 12.09 & UAU & 9.78 \\
\hline 421 & $203308.09+411526.9$ & $20330809+4115270$ & 0.16 & $16.24 \pm 0.11$ & $15.22 \pm 0.08$ & 14.45 & BAU & 5.62 \\
\hline 422 & $203308.21+410626.4$ & $20330817+4106265$ & 0.57 & $13.28 \pm 0.02$ & $12.12 \pm 0.04$ & $11.70 \pm 0.02$ & AAA & 8.89 \\
\hline 423 & $203308.21+411304.0$ & $20330821+4113045$ & 0.23 & $14.45 \pm 0.05$ & $15.34 \pm 0.21$ & $14.72 \pm 0.18$ & ECC & 5.03 \\
\hline 424 & $203308.23+411823.1$ & $20330824+4118235$ & 0.20 & $15.41 \pm 0.06$ & $14.26 \pm 0.06$ & $13.71 \pm 0.05$ & AAA & N/A \\
\hline 425 & $203308.25+411513.1$ & - & - & - & - & - & - & - \\
\hline 426 & $203308.29+411543.0$ & $20330833+4115432$ & 0.55 & $13.68 \pm 0.03$ & $13.10 \pm 0.03$ & $12.85 \pm 0.04$ & AAA & 0.18 \\
\hline 427 & $203308.33+411421.1$ & $20330834+4114214$ & 0.06 & $15.72 \pm 0.06$ & $14.28 \pm 0.03$ & $13.76 \pm 0.05$ & AAA & 8.52 \\
\hline 428 & $203308.50+411734.6$ & $20330848+4117347$ & 0.36 & $16.37 \pm 0.11$ & $15.12 \pm 0.08$ & $14.61 \pm 0.11$ & BAB & 4.98 \\
\hline 429 & $203308.53+411328.1$ & - & - & - & - & - & - & - \\
\hline 430 & $203308.56+411435.9$ & - & - & - & - & - & - & - \\
\hline 431 & $203308.59+411915.1$ & $20330859+4119154$ & 0.02 & $13.17 \pm 0.02$ & $11.97 \pm 0.01$ & $10.93 \pm 0.01$ & AAA & N/A \\
\hline 432 & $203308.63+411514.4$ & $20330865+4115146$ & 0.22 & $14.15 \pm 0.04$ & $12.76 \pm 0.03$ & $12.20 \pm 0.02$ & AAA & N/A \\
\hline 433 & $203308.76+411318.5$ & $20330879+4113182$ & 0.66 & $7.11 \pm 0.01$ & $6.54 \pm 0.01$ & $6.23 \pm 0.01$ & AAA & N/A \\
\hline 434 & $203308.80+411257.4$ & - & - & - & - & - & - & - \\
\hline 435 & $203308.84+411214.8$ & $20330880+4112151$ & 0.66 & 15.72 & $14.99 \pm 0.10$ & 13.08 & UAU & 11.35 \\
\hline 436 & $203308.84+411317.1$ & - & - & - & - & - & - & - \\
\hline 437 & $203308.89+411327.7$ & - & - & - & - & - & - & - \\
\hline 438 & $203308.90+411243.5$ & - & - & - & - & - & - & - \\
\hline 439 & $203308.91+412227.3$ & $20330892+4122276$ & 0.06 & $15.73 \pm 0.06$ & $14.60 \pm 0.05$ & $14.31 \pm 0.09$ & AAA & 4.36 \\
\hline 440 & $203308.95+411705.7$ & - & - & - & - & - & - & - \\
\hline 441 & $203308.96+411101.9$ & $20330896+4111024$ & 0.35 & $15.84 \pm 0.07$ & $14.56 \pm 0.05$ & $14.01 \pm 0.06$ & AAA & 3.03 \\
\hline 442 & $203308.97+411537.5$ & - & - & - & - & - & - & - \\
\hline 443 & $203309.01+411331.0$ & - & - & - & - & - & - & - \\
\hline 444 & $203309.06+411227.8$ & - & - & - & - & - & - & - \\
\hline 445 & $203309.12+411308.6$ & $20330911+4113094$ & 0.68 & $14.24 \pm 0.16$ & $13.11 \pm 0.13$ & 11.93 & $\mathrm{CBU}$ & N/A \\
\hline 446 & $203309.15+411343.2$ & $20330915+4113434$ & 0.11 & $10.83 \pm 0.03$ & $10.22 \pm 0.03$ & $9.88 \pm 0.02$ & AAA & 5.87 \\
\hline 447 & $203309.26+411302.1$ & - & - & - & - & - & - & - \\
\hline 448 & $203309.30+411618.6$ & $20330929+4116188$ & 0.34 & 16.14 & 14.54 & $14.24 \pm 0.09$ & UUA & 5.28 \\
\hline 449 & $203309.32+411250.6$ & $20330944+4112510$ & 1.76 & 11.87 & 11.14 & $12.88 \pm 0.06$ & UUA & 35.41 \\
\hline 450 & $203309.32+411428.9$ & $20330936+4114290$ & 0.44 & $11.88 \pm 0.02$ & $11.17 \pm 0.02$ & $10.81 \pm 0.02$ & AAA & 13.50 \\
\hline 451 & $203309.38+411347.0$ & - & - & - & - & - & - & - \\
\hline 452 & $203309.42+411320.3$ & - & - & - & - & - & - & - \\
\hline 453 & $203309.45+411258.3$ & - & - & - & - & - & - & - \\
\hline 454 & $203309.53+412109.9$ & $20330954+4121102$ & 0.16 & $15.61 \pm 0.05$ & $14.25 \pm 0.04$ & $13.72 \pm 0.05$ & AAA & N/A \\
\hline 455 & $203309.59+411308.0$ & - & - & - & - & - & - & - \\
\hline
\end{tabular}


Table 2. continued.

\begin{tabular}{|c|c|c|c|c|c|c|c|c|}
\hline \multirow{2}{*}{$\begin{array}{l}N_{\mathrm{x}} \\
\#\end{array}$} & \multicolumn{2}{|c|}{$\overline{\text { X-ray - 2MASS counterpart }}$} & \multirow{2}{*}{$\begin{array}{l}\text { Off. } \\
\left({ }^{\prime \prime}\right)\end{array}$} & \multicolumn{4}{|c|}{ 2MASS photometry } & \multirow{2}{*}{$\begin{array}{l}A_{\mathrm{v}} \\
(\mathrm{mag})\end{array}$} \\
\hline & CXOAC J+ & 2MASS J+ & & $J$ & $H$ & $K_{\mathrm{s}}$ & Ph.qual & \\
\hline 456 & $203309.60+411300.4$ & $20330960+4113005$ & 0.20 & $8.65 \pm 0.01$ & $8.14 \pm 0.01$ & $7.76 \pm 0.01$ & AAE & 9.20 \\
\hline 457 & $203309.60+411429.9$ & - & - & - & - & - & - & - \\
\hline 458 & $203309.63+412130.4$ & - & - & - & - & - & - & - \\
\hline 459 & $203309.67+412214.3$ & $20330984+4122143$ & 2.53 & $15.87 \pm 0.09$ & $14.55 \pm 0.06$ & $14.22 \pm 0.08$ & AAA & 8.06 \\
\hline 460 & $203309.73+411250.6$ & $20330965+4112508$ & 1.21 & 13.06 & $14.06 \pm 0.13$ & 11.80 & UBU & 9.80 \\
\hline 461 & $203309.78+410902.2$ & $20330978+4109029$ & 0.52 & $15.71 \pm 0.07$ & $14.30 \pm 0.06$ & $13.76 \pm 0.06$ & AAA & 14.58 \\
\hline 462 & $203309.85+411247.8$ & - & - & - & - & - & - & - \\
\hline 463 & $203309.92+411139.5$ & - & - & - & - & - & - & - \\
\hline 464 & $203309.92+411756.2$ & $20330992+4117563$ & 0.17 & $14.83 \pm 0.03$ & $13.54 \pm 0.03$ & $12.79 \pm 0.02$ & AAA & 10.20 \\
\hline 465 & $203309.93+411325.9$ & $20330994+4113260$ & 0.20 & $15.66 \pm 0.09$ & 13.10 & 12.68 & AUU & 5.40 \\
\hline 466 & $203309.95+410856.9$ & $20330996+4108571$ & 0.03 & $16.55 \pm 0.14$ & $15.16 \pm 0.08$ & $14.50 \pm 0.10$ & BAA & 7.70 \\
\hline 467 & $203310.02+412042.2$ & - & - & - & - & - & - & - \\
\hline 468 & $203310.03+411429.6$ & - & - & - & - & - & - & - \\
\hline 469 & $203310.04+411743.2$ & - & - & - & - & - & - & - \\
\hline 470 & $203310.05+411301.6$ & - & - & - & - & - & - & - \\
\hline 471 & $203310.07+411000.8$ & $20331014+4110026$ & 1.88 & $10.49 \pm 0.02$ & $9.71 \pm 0.01$ & $9.37 \pm 0.01$ & AAE & 3.06 \\
\hline 472 & $203310.10+411414.4$ & - & - & - & - & - & - & - \\
\hline 473 & $203310.11+411337.1$ & - & - & - & - & - & - & - \\
\hline 474 & $203310.12+411239.7$ & $20331014+4112398$ & 0.19 & $14.90 \pm 0.04$ & $13.75 \pm 0.04$ & $13.26 \pm 0.04$ & AAA & 4.49 \\
\hline 475 & $203310.17+411545.3$ & $20331018+4115455$ & 0.11 & $14.10 \pm 0.03$ & $12.90 \pm 0.04$ & $12.37 \pm 0.03$ & AAA & 7.64 \\
\hline 476 & $203310.18+411339.4$ & $20331016+4113394$ & 0.39 & $14.49 \pm 0.02$ & $13.26 \pm 0.05$ & $12.78 \pm 0.04$ & AAA & 6.17 \\
\hline 477 & $203310.21+411322.4$ & - & - & - & - & - & - & - \\
\hline 478 & $203310.23+411533.1$ & - & - & - & - & - & - & - \\
\hline 479 & $203310.26+411413.3$ & $20331027+4114136$ & 0.04 & $14.99 \pm 0.03$ & $13.59 \pm 0.03$ & $13.06 \pm 0.03$ & AAA & 7.48 \\
\hline 480 & $203310.29+412205.1$ & $20331029+4122055$ & 0.10 & $13.46 \pm 0.03$ & $12.52 \pm 0.02$ & $12.12 \pm 0.02$ & AAA & 14.32 \\
\hline 481 & $203310.45+410925.5$ & $20331047+4109257$ & 0.11 & 16.52 & $15.52 \pm 0.12$ & $14.68 \pm 0.12$ & UBB & 7.45 \\
\hline 482 & $203310.56+411328.4$ & $20331059+4113285$ & 0.24 & $14.98 \pm 0.03$ & $13.62 \pm 0.03$ & $13.05 \pm 0.04$ & AAA & N/A \\
\hline 483 & $203310.57+412102.3$ & $20331060+4121041$ & 1.61 & $15.39 \pm 0.08$ & $14.31 \pm 0.09$ & $13.97 \pm 0.06$ & AAA & N/A \\
\hline 484 & $203310.58+411538.7$ & - & - & - & - & - & - & - \\
\hline 485 & $203310.60+411323.8$ & - & - & - & - & - & - & - \\
\hline 486 & $203310.64+411748.8$ & - & - & - & - & - & - & - \\
\hline 487 & $203310.71+411339.6$ & - & - & - & - & - & - & - \\
\hline 488 & $203310.72+411507.9$ & $20331074+4115081$ & 0.33 & $6.47 \pm 0.01$ & $5.90 \pm 0.01$ & $5.57 \pm \mathrm{N} / \mathrm{A}$ & AAA & N/A \\
\hline 489 & $203310.73+411502.2$ & - & - & - & - & - & - & - \\
\hline 490 & $203310.81+411256.4$ & $20331084+4112568$ & 0.42 & $15.78 \pm 0.09$ & $14.45 \pm 0.08$ & 13.61 & AAU & 6.49 \\
\hline 491 & $203310.93+410953.3$ & $20331095+4109537$ & 0.16 & $16.38 \pm 0.12$ & $14.90 \pm 0.07$ & $14.42 \pm 0.09$ & BAA & N/A \\
\hline 492 & $203310.99+411241.8$ & - & - & - & - & - & - & - \\
\hline 493 & $203311.05+411032.0$ & $20331106+4110321$ & 0.16 & $10.17 \pm 0.02$ & $9.60 \pm 0.01$ & $9.28 \pm 0.01$ & AAA & N/A \\
\hline 494 & $203311.07+411318.0$ & - & - & - & - & - & - & - \\
\hline 495 & $203311.09+411445.4$ & $20331108+4114457$ & 0.25 & $13.93 \pm 0.01$ & $13.34 \pm 0.03$ & $13.24 \pm 0.05$ & AAA & 9.77 \\
\hline 496 & $203311.13+411640.6$ & - & - & - & - & - & - & - \\
\hline 497 & $203311.22+410833.9$ & $20331123+4108338$ & 0.39 & $15.27 \pm 0.04$ & $13.98 \pm 0.02$ & $13.44 \pm 0.04$ & AAA & N/A \\
\hline 498 & $203311.29+411711.4$ & - & - & - & - & - & - & - \\
\hline 499 & $203311.32+411729.0$ & $20331134+4117294$ & 0.24 & $15.31 \pm 0.05$ & $14.06 \pm 0.04$ & $13.55 \pm 0.05$ & AAA & 8.17 \\
\hline 500 & $203311.44+411256.7$ & $20331145+4112568$ & 0.10 & $14.87 \pm 0.05$ & $13.60 \pm 0.05$ & 12.97 & AAU & 9.39 \\
\hline 501 & $203311.47+411348.0$ & $20331148+4113481$ & 0.14 & $16.11 \pm 0.09$ & $14.40 \pm 0.05$ & $13.98 \pm 0.06$ & AAA & 9.16 \\
\hline 502 & $203311.51+411238.8$ & - & - & - & - & - & - & - \\
\hline 503 & $203311.52+410718.9$ & $20331151+4107197$ & 0.63 & $15.71 \pm 0.06$ & $14.53 \pm 0.06$ & $14.28 \pm 0.08$ & AAA & 5.52 \\
\hline 504 & $203311.59+411243.0$ & - & - & - & - & - & - & - \\
\hline 505 & $203311.60+411615.8$ & $20331157+4116169$ & 0.96 & $16.94 \pm 0.19$ & $15.43 \pm 0.09$ & $14.94 \pm 0.16$ & $\mathrm{CAC}$ & 8.29 \\
\hline 506 & $203311.63+411646.4$ & $20331162+4116468$ & 0.29 & $14.94 \pm 0.05$ & $13.49 \pm 0.05$ & 12.77 & AAU & N/A \\
\hline 507 & $203311.79+411149.9$ & $20331180+4111502$ & 0.16 & $16.25 \pm 0.13$ & $14.89 \pm 0.11$ & $14.33 \pm 0.10$ & BAA & 11.08 \\
\hline 508 & $203311.87+411245.3$ & $20331183+4112455$ & 0.66 & $15.06 \pm 0.16$ & $13.66 \pm 0.08$ & $13.25 \pm 0.07$ & CAA & N/A \\
\hline 509 & $203311.89+411213.9$ & $20331189+4112139$ & 0.24 & $15.11 \pm 0.04$ & $13.86 \pm 0.03$ & $13.47 \pm 0.04$ & AAA & 6.84 \\
\hline 510 & $203312.04+412023.1$ & $20331204+4120235$ & 0.15 & $12.73 \pm 0.03$ & $11.96 \pm 0.03$ & $11.56 \pm 0.02$ & AAA & 6.83 \\
\hline 511 & $203312.05+411649.2$ & $20331206+4116495$ & 0.11 & $15.53 \pm 0.05$ & $14.19 \pm 0.04$ & 13.51 & AAU & N/A \\
\hline 512 & $203312.20+411014.2$ & - & - & - & - & - & - & - \\
\hline 513 & $203312.25+411104.6$ & - & - & - & - & - & - & - \\
\hline 514 & $203312.50+411502.0$ & $20331252+4115021$ & 0.22 & $12.28 \pm 0.02$ & $11.21 \pm 0.02$ & $10.43 \pm 0.01$ & AAA & 2.45 \\
\hline 515 & $203312.50+411150.1$ & - & - & - & - & - & - & - \\
\hline 516 & $203312.57+411453.3$ & $20331259+4114532$ & 0.39 & $16.15 \pm 0.11$ & $14.40 \pm 0.04$ & $13.53 \pm 0.06$ & BAA & 10.13 \\
\hline 517 & $203312.60+411539.4$ & $20331261+4115395$ & 0.09 & $16.01 \pm 0.09$ & $14.57 \pm 0.05$ & $14.21 \pm 0.08$ & AAA & 10.72 \\
\hline 518 & $203312.62+411635.1$ & $20331265+4116353$ & 0.33 & 16.77 & $15.25 \pm 0.08$ & $14.49 \pm 0.10$ & UAA & 1.28 \\
\hline 519 & $203312.66+411913.6$ & - & - & - & - & - & - & - \\
\hline 520 & $203312.73+411132.6$ & $20331273+4111330$ & 0.16 & $13.98 \pm 0.02$ & $12.67 \pm 0.02$ & $11.99 \pm 0.02$ & AAA & 12.31 \\
\hline
\end{tabular}


Table 2. continued.

\begin{tabular}{|c|c|c|c|c|c|c|c|c|}
\hline \multirow{2}{*}{$\begin{array}{l}N_{\mathrm{x}} \\
\#\end{array}$} & \multicolumn{2}{|c|}{ X-ray - 2MASS counterpart } & \multirow{2}{*}{$\begin{array}{l}\text { Off. } \\
\left({ }^{\prime \prime}\right)\end{array}$} & \multicolumn{4}{|c|}{ 2MASS photometry } & \multirow{2}{*}{$\begin{array}{l}\begin{array}{l}A_{\mathrm{v}} \\
(\mathrm{mag})\end{array} \\
\text { (a) }\end{array}$} \\
\hline & CXOAC J+ & 2MASS J+ & & $J$ & $\bar{H}$ & $K_{\mathrm{s}}$ & Ph. qual & \\
\hline 521 & $203312.74+411439.3$ & - & - & - & - & - & - & - \\
\hline 522 & $203312.86+412115.8$ & $20331289+4121171$ & 1.06 & $16.00 \pm 0.08$ & $14.66 \pm 0.05$ & $14.31 \pm 0.09$ & AAA & N/A \\
\hline 523 & $203312.87+411154.4$ & - & - & - & - & - & - & - \\
\hline 524 & $203312.97+411257.5$ & $20331287+4112575$ & 1.56 & 13.91 & $14.72 \pm 0.08$ & 12.67 & UAU & 0.93 \\
\hline 525 & $203313.03+411539.0$ & $20331304+4115393$ & 0.09 & $15.58 \pm 0.05$ & $14.20 \pm 0.04$ & $13.62 \pm 0.05$ & AAA & 7.65 \\
\hline 526 & $203313.04+411311.5$ & $20331307+4113119$ & 0.40 & $15.20 \pm 0.17$ & $14.04 \pm 0.10$ & $13.73 \pm 0.08$ & CAA & N/A \\
\hline 527 & $203313.08+411030.1$ & $20331305+4110311$ & 0.92 & $16.44 \pm 0.12$ & $15.01 \pm 0.07$ & $14.49 \pm 0.10$ & BAA & 7.86 \\
\hline 528 & $203313.16+411338.6$ & $20331320+4113389$ & 0.45 & $15.25 \pm 0.12$ & $13.98 \pm 0.08$ & $13.56 \pm 0.05$ & BAA & 11.21 \\
\hline 529 & $203313.23+411319.1$ & - & - & - & - & - & - & - \\
\hline 530 & $203313.24+411328.4$ & $20331326+4113287$ & 0.12 & $8.98 \pm 0.03$ & $8.35 \pm 0.01$ & $8.01 \pm 0.01$ & AAA & 7.43 \\
\hline 531 & $203313.25+411727.2$ & $20331327+4117275$ & 0.22 & 16.26 & $15.19 \pm 0.09$ & $14.70 \pm 0.12$ & UAB & N/A \\
\hline 532 & $203313.38+411110.8$ & $20331334+4111111$ & 0.77 & $15.97 \pm 0.09$ & $14.54 \pm 0.06$ & $14.10 \pm 0.07$ & AAA & 6.85 \\
\hline 533 & $203313.40+411233.6$ & $20331339+4112337$ & 0.37 & $16.59 \pm 0.13$ & $15.01 \pm 0.07$ & $14.70 \pm 0.13$ & BAB & 14.74 \\
\hline 534 & $203313.54+411948.8$ & $20331362+4119494$ & 1.06 & $14.84 \pm 0.03$ & $13.70 \pm 0.02$ & $13.26 \pm 0.04$ & AAA & N/A \\
\hline 535 & $203313.60+412004.8$ & $20331366+4120052$ & 0.78 & $15.66 \pm 0.07$ & $14.61 \pm 0.05$ & $13.96 \pm 0.07$ & AAA & N/A \\
\hline 536 & $203313.62+411749.2$ & $20331359+4117497$ & 0.63 & $15.10 \pm 0.04$ & $14.20 \pm 0.04$ & $13.95 \pm 0.07$ & AAA & N/A \\
\hline 537 & $203313.67+411305.4$ & $20331369+4113057$ & 0.15 & $9.03 \pm 0.02$ & $8.56 \pm 0.02$ & $8.28 \pm 0.01$ & AAA & N/A \\
\hline 538 & $203313.78+411028.0$ & $20331377+4110294$ & 1.22 & $16.12 \pm 0.09$ & $14.77 \pm 0.07$ & $14.15 \pm 0.08$ & AAA & 6.11 \\
\hline 539 & $203313.85+411715.1$ & $20331385+4117154$ & 0.23 & $16.02 \pm 0.08$ & $15.01 \pm 0.07$ & $14.62 \pm 0.11$ & AAA & N/A \\
\hline 540 & $203313.87+411259.0$ & $20331382+4113001$ & 1.22 & 11.83 & $13.47 \pm 0.11$ & 11.01 & UBU & 9.91 \\
\hline 541 & $203313.88+411345.4$ & $20331387+4113458$ & 0.26 & 16.49 & $15.48 \pm 0.11$ & $14.35 \pm 0.09$ & UBA & 9.67 \\
\hline 542 & $203314.05+411626.9$ & $20331406+4116270$ & 0.08 & $16.41 \pm 0.12$ & $14.68 \pm 0.05$ & $13.65 \pm 0.05$ & BAA & 5.91 \\
\hline 543 & $203314.09+410925.6$ & $20331411+4109264$ & 0.51 & $15.90 \pm 0.07$ & $14.55 \pm 0.05$ & $14.06 \pm 0.07$ & AAA & 5.17 \\
\hline 544 & $203314.11+412021.6$ & $20331411+4120218$ & 0.15 & $7.25 \pm 0.01$ & $6.82 \pm 0.01$ & $6.61 \pm 0.01$ & AAA & N/A \\
\hline 545 & $203314.13+411446.6$ & - & - & - & - & - & - & - \\
\hline 546 & $203314.15+411450.2$ & $20331416+4114504$ & 0.01 & $13.27 \pm 0.02$ & $12.24 \pm 0.02$ & $11.83 \pm 0.02$ & AAA & 1.12 \\
\hline 547 & $203314.16+412054.9$ & - & - & - & - & - & - & - \\
\hline 548 & $203314.22+410852.7$ & $20331419+4108523$ & 0.83 & $16.69 \pm 0.15$ & $15.49 \pm 0.10$ & $15.19 \pm 0.18$ & BAC & N/A \\
\hline 549 & $203314.24+411227.8$ & $20331428+4112283$ & 0.48 & $15.04 \pm 0.06$ & $13.61 \pm 0.06$ & $13.14 \pm 0.06$ & AAA & N/A \\
\hline 550 & $203314.25+411236.7$ & $20331421+4112372$ & 0.69 & $15.47 \pm 0.06$ & $14.21 \pm 0.05$ & $13.70 \pm 0.06$ & AAA & 7.87 \\
\hline 551 & $203314.31+411221.2$ & $20331434+4112218$ & 0.51 & $15.39 \pm 0.06$ & $13.91 \pm 0.04$ & $13.08 \pm 0.04$ & AAA & N/A \\
\hline 552 & $203314.32+411932.8$ & $20331433+4119331$ & 0.11 & $10.19 \pm 0.02$ & $9.57 \pm 0.02$ & $9.24 \pm 0.02$ & AAA & 6.98 \\
\hline 553 & $203314.38+411223.5$ & - & - & - & - & - & - & - \\
\hline 554 & $203314.39+411901.0$ & - & - & - & - & - & - & - \\
\hline 555 & $203314.42+411923.5$ & $20331443+4119238$ & 0.06 & $11.66 \pm 0.02$ & $10.83 \pm 0.02$ & $10.26 \pm$ & AAA & 8.99 \\
\hline 556 & $203314.44+410633.3$ & $20331442+4106320$ & 1.56 & 15.61 & $14.60 \pm 0.08$ & 14.02 & UAU & N/A \\
\hline 557 & $203314.47+411856.2$ & - & - & - & - & - & - & - \\
\hline 558 & $203314.50+411253.9$ & $20331451+4112542$ & 0.08 & $15.92 \pm 0.08$ & $14.50 \pm 0.06$ & $14.19 \pm 0.08$ & AAA & N/A \\
\hline 559 & $203314.58+411837.1$ & - & - & - & - & - & - & - \\
\hline 560 & $203314.69+410944.1$ & - & - & - & - & - & - & - \\
\hline 561 & $203314.76+412012.5$ & - & - & - & - & - & - & - \\
\hline 562 & $203314.76+411841.4$ & $20331476+4118416$ & 0.23 & $7.21 \pm 0.02$ & $6.76 \pm 0.01$ & $6.57 \pm 0.01$ & AAA & 4.93 \\
\hline 563 & $203314.81+411421.0$ & $20331480+4114215$ & 0.42 & $16.78 \pm 0.16$ & $15.34 \pm 0.10$ & $14.84 \pm 0.15$ & $\mathrm{CAB}$ & 4.95 \\
\hline 564 & $203314.84+410831.4$ & $20331492+4108317$ & 0.99 & $15.08 \pm 0.04$ & $13.81 \pm 0.03$ & $13.42 \pm 0.04$ & AAA & 8.56 \\
\hline 565 & $203314.87+410957.6$ & $20331487+4109572$ & 0.66 & $16.82 \pm 0.16$ & $14.93 \pm 0.07$ & $14.25 \pm 0.07$ & CAA & 6.46 \\
\hline 566 & $203314.92+411719.4$ & - & - & - & - & - & - & - \\
\hline 567 & $203314.97+411556.8$ & $20331500+4115572$ & 0.26 & $15.57 \pm 0.05$ & $14.09 \pm 0.03$ & $13.48 \pm 0.04$ & AAA & N/A \\
\hline 568 & $203315.07+411850.3$ & $20331508+4$ & 0.15 & \pm 0.01 & $5.72 \pm 0.01$ & $5.50 \pm 0.01$ & AAA & 12.75 \\
\hline 569 & $203315.11+411310.5$ & $20331513+4113106$ & 0.28 & $16.03 \pm 0.11$ & $14.46 \pm 0.06$ & $13.77 \pm$ & BAA & 9.97 \\
\hline 570 & $203315.12+411903.3$ & - & - & - & - & - & - & - \\
\hline 571 & $203315.18+411530.8$ & $20331519+4115311$ & 0.12 & $14.80 \pm 0.04$ & $13.46 \pm 0.03$ & $12.95 \pm 0.03$ & AAA & N/A \\
\hline 572 & $203315.21+411327.5$ & $20331523+4113277$ & 0.22 & $15.72 \pm 0.09$ & $14.28 \pm 0.08$ & $13.66 \pm 0.06$ & AAA & N/A \\
\hline 573 & $203315.28+411522.6$ & $20331529+4115229$ & 0.11 & $15.36 \pm 0.04$ & $13.85 \pm 0.01$ & $13.34 \pm 0.03$ & AAA & N/A \\
\hline 574 & $203315.31+411855.6$ & - & - & - & - & - & - & - \\
\hline 575 & $203315.33+411029.9$ & $20331532+4110297$ & 0.58 & $6 \pm($ & $13.93 \pm 0.04$ & $13.52 \pm$ & AAA & N/A \\
\hline 576 & $203315.38+411751.5$ & $20331541+4117518$ & 0.35 & $15.37 \pm 0.05$ & $14.18 \pm 0.04$ & $13.82 \pm 0.06$ & AAA & N/A \\
\hline 577 & $203315.39+411338.4$ & $20331539+4113389$ & 0.25 & $15.19 \pm 0.04$ & $13.88 \pm 0.03$ & $13.40 \pm 0.04$ & AAA & 4.15 \\
\hline 578 & $203315.40+411329.6$ & - & - & - & - & - & - & - \\
\hline 579 & $203315.43+411816.0$ & $20331546+4118162$ & 0.33 & $15.50 \pm 0.07$ & $14.31 \pm 0.07$ & $13.80 \pm 0.06$ & AAA & N/A \\
\hline 580 & $203315.45+411856.7$ & - & - & - & - & - & - & - \\
\hline 581 & $203315.55+412133.3$ & - & - & - & - & - & - & - \\
\hline 582 & $203315.63+411851.4$ & - & - & - & - & - & - & - \\
\hline 583 & $203315.68+411319.4$ & - & - & - & - & - & - & - \\
\hline 584 & $203315.70+412017.0$ & $20331571+4120172$ & 0.08 & $9.33 \pm 0.02$ & $8.94 \pm 0.01$ & $8.73 \pm 0.02$ & AAA & 6.99 \\
\hline 585 & $203315.76+410633.1$ & $20331562+4106322$ & 2.59 & $15.81 \pm 0.07$ & $14.71 \pm 0.08$ & $14.15 \pm 0.07$ & AAA & 7.48 \\
\hline
\end{tabular}


Table 2. continued.

\begin{tabular}{|c|c|c|c|c|c|c|c|c|}
\hline \multirow{2}{*}{$\begin{array}{l}N_{\mathrm{x}} \\
\#\end{array}$} & \multicolumn{2}{|c|}{ X-ray - 2MASS counterpart } & \multirow{2}{*}{$\begin{array}{l}\text { Off. } \\
\left({ }^{\prime \prime}\right)\end{array}$} & \multicolumn{4}{|c|}{ 2MASS photometry } & \multirow{2}{*}{$\begin{array}{l}\begin{array}{l}A_{\mathrm{v}} \\
(\mathrm{mag})\end{array} \\
\text { (a) }\end{array}$} \\
\hline & CXOAC J+ & 2MASS J+ & & $J$ & $\bar{H}$ & $K_{\mathrm{s}}$ & Ph. qual & \\
\hline 586 & $203315.80+411510.2$ & $20331579+4115107$ & 0.29 & 15.32 & 14.21 & $14.27 \pm 0.10$ & $\overline{\text { UUA }}$ & N/A \\
\hline 587 & $203315.82+411225.8$ & $20331584+4112264$ & 0.44 & $16.33 \pm 0.11$ & $15.04 \pm 0.07$ & $14.44 \pm 0.09$ & AAA & 10.41 \\
\hline 588 & $203315.84+412115.0$ & - & - & - & - & - & - & - \\
\hline 589 & $203315.89+411623.3$ & $20331589+4116239$ & 0.41 & $15.95 \pm 0.08$ & $14.72 \pm 0.05$ & $14.21 \pm 0.08$ & AAA & 18.23 \\
\hline 590 & $203315.90+412025.3$ & $20331589+4120254$ & 0.35 & $12.60 \pm 0.04$ & $11.99 \pm 0.02$ & 11.73 & AAU & 6.55 \\
\hline 591 & $203316.17+411245.3$ & $20331619+4112459$ & 0.34 & $14.08 \pm 0.04$ & $13.10 \pm 0.04$ & $12.77 \pm 0.03$ & AAA & N/A \\
\hline 592 & $203316.21+411721.8$ & $20331622+4117220$ & 0.01 & $15.19 \pm 0.05$ & $13.82 \pm 0.04$ & $13.37 \pm 0.05$ & AAA & 7.35 \\
\hline 593 & $203316.21+411440.3$ & $20331624+4114406$ & 0.44 & $14.35 \pm 0.05$ & $12.90 \pm 0.08$ & $12.48 \pm 0.04$ & AAA & 7.06 \\
\hline 594 & $203316.22+412015.5$ & $20331623+4120158$ & 0.13 & $14.48 \pm 0.07$ & $13.51 \pm 0.06$ & $13.03 \pm 0.05$ & AAA & 7.48 \\
\hline 595 & $203316.29+412143.7$ & - & - & - & - & - & - & - \\
\hline 596 & $203316.34+411858.9$ & - & - & - & - & - & - & - \\
\hline 597 & $203316.36+411902.1$ & $20331634+4119017$ & 0.72 & $8.84 \pm 0.01$ & $8.42 \pm 0.04$ & $8.24 \pm 0.01$ & AAA & 10.15 \\
\hline 598 & $203316.44+411659.2$ & $20331645+4116597$ & 0.23 & $15.16 \pm 0.06$ & $13.85 \pm 0.07$ & $13.27 \pm 0.05$ & AAA & 7.19 \\
\hline 599 & $203316.45+410924.9$ & $20331647+4109245$ & 0.62 & 16.99 & $15.32 \pm 0.09$ & $14.80 \pm 0.12$ & UAB & 4.88 \\
\hline 600 & $203316.50+411653.9$ & - & - & - & - & - & - & - \\
\hline 601 & $203316.52+410703.7$ & $20331653+4107036$ & 0.41 & $14.81 \pm 0.04$ & $13.77 \pm 0.03$ & $13.42 \pm 0.04$ & AAA & 6.62 \\
\hline 602 & $203316.57+411703.9$ & $20331658+4117039$ & 0.25 & $12.47 \pm 0.03$ & $11.60 \pm 0.03$ & $11.25 \pm 0.04$ & AAA & 7.45 \\
\hline 603 & $203316.60+411258.8$ & $20331660+4112589$ & 0.17 & $14.88 \pm 0.08$ & $13.60 \pm 0.06$ & $13.18 \pm 0.05$ & AAA & 4.36 \\
\hline 604 & $203316.64+411542.2$ & $20331667+4115423$ & 0.35 & 16.39 & $15.15 \pm 0.08$ & $14.70 \pm 0.13$ & UAB & N/A \\
\hline 605 & $203316.66+411404.4$ & $20331663+4114053$ & 0.92 & 16.50 & $14.77 \pm 0.07$ & $14.31 \pm 0.09$ & BAA & 0.64 \\
\hline 606 & $203316.72+411833.1$ & - & - & - & - & - & - & - \\
\hline 607 & $203316.73+411129.3$ & $20331675+4111298$ & 0.26 & $16.60 \pm 0.14$ & 15.03 & 14.44 & BUU & N/A \\
\hline 608 & $203316.83+411056.8$ & $20331682+4110575$ & 0.53 & $14.54 \pm 0.04$ & $13.27 \pm 0.03$ & $12.72 \pm 0.03$ & AAA & 3.34 \\
\hline 609 & $203317.08+411659.7$ & $20331712+4116599$ & 0.44 & $14.86 \pm 0.09$ & $13.72 \pm 0.09$ & $13.46 \pm 0.06$ & AAA & 41.63 \\
\hline 610 & $203317.12+411506.6$ & $20331714+4115067$ & 0.23 & $16.36 \pm 0.11$ & $15.00 \pm 0.07$ & $14.27 \pm 0.08$ & BAA & N/A \\
\hline 611 & $203317.31+411342.0$ & $20331733+4113421$ & 0.25 & $15.69 \pm 0.06$ & $14.74 \pm 0.07$ & $14.21 \pm 0.08$ & AAA & 5.90 \\
\hline 612 & $203317.38+411653.9$ & - & - & - & - & - & - & - \\
\hline 613 & $203317.40+411453.8$ & $20331740+4114541$ & 0.01 & $11.35 \pm 0.02$ & $10.78 \pm 0.01$ & $10.48 \pm 0.01$ & AAA & 6.41 \\
\hline 614 & $203317.40+411238.4$ & $20331740+4112387$ & 0.26 & $10.23 \pm 0.02$ & $9.68 \pm 0.01$ & $9.43 \pm 0.01$ & AAA & 9.41 \\
\hline 615 & $203317.47+411709.0$ & $20331748+4117093$ & 0.02 & $8.35 \pm 0.02$ & $7.89 \pm 0.01$ & $7.65 \pm 0.01$ & AAA & 7.23 \\
\hline 616 & $203317.51+410724.3$ & $20331744+4107242$ & 1.27 & $16.63 \pm 0.14$ & $15.39 \pm 0.11$ & 14.52 & $\mathrm{BBU}$ & 11.43 \\
\hline 617 & $203317.53+411800.7$ & - & - & - & - & - & - & - \\
\hline 618 & $203317.55+412041.1$ & $20331754+4$ & 0.41 & 16. & $14.98 \pm 0.07$ & .10 & AAA & 9.41 \\
\hline 619 & $203317.63+412022.0$ & $20331767+4120221$ & 0.46 & $3 \pm 0$ & $14.39 \pm 0.05$ & $14.02 \pm 0.07$ & AAA & 45.95 \\
\hline 620 & $203317.74+411304.7$ & $20331774+4113047$ & 0.23 & $13.44 \pm 0.01$ & $12.23 \pm 0.01$ & $11.42 \pm$ & AAA & 8.69 \\
\hline 621 & $203317.76+411753.0$ & $20331777+4117532$ & 0.06 & 17.36 & $15.53 \pm 0.12$ & 15.47 & UBU & 0.62 \\
\hline 622 & $203317.83+411323.3$ & $20331787+4113240$ & 0.60 & $13.52 \pm 0.03$ & $12.58 \pm 0.03$ & $12.20 \pm 0.03$ & AAA & 6.86 \\
\hline 623 & $203317.95+411917.9$ & $20331800+4119181$ & 0.76 & $14.22 \pm 0.02$ & $13.30 \pm 0.02$ & $12.93 \pm 0.03$ & AAA & N/A \\
\hline 624 & $203317.97+410749.6$ & $20331807+4107499$ & 1.43 & $16.74 \pm 0.15$ & $15.19 \pm 0.08$ & $14.73 \pm 0.12$ & BAB & 39.70 \\
\hline 625 & $203317.99+411830.9$ & $20331798+4118311$ & 0.23 & $7.16 \pm 0.01$ & $6.79 \pm 0.01$ & $6.58 \pm 0.01$ & AAA & 7.22 \\
\hline 626 & $203318.06+412136.3$ & $20331803+4121366$ & 0.55 & $8.74 \pm 0.01$ & $8.31 \pm 0.01$ & $8.11 \pm 0.01$ & AAA & N/A \\
\hline 627 & $203318.06+412136.4$ & $20331803+4121366$ & 0.55 & $8.74 \pm 0.01$ & $8.31 \pm 0.01$ & $8.11 \pm 0.01$ & AAA & 5.19 \\
\hline 628 & $203318.06+411714.4$ & $20331809+4117146$ & 0.45 & $15.58 \pm 0.37$ & $14.29 \pm 0.17$ & $14.23 \pm 0.12$ & DCB & 5.24 \\
\hline 629 & $203318.08+411731.1$ & $20331811+4117315$ & 0.35 & $12.26 \pm 0.02$ & $11.44 \pm 0.02$ & $11.06 \pm 0.01$ & AAA & 14.35 \\
\hline 630 & $203318.08+411731.2$ & $20331811+4117315$ & 0.35 & $12.26 \pm 0.02$ & $11.44 \pm 0.02$ & $11.06 \pm 0.01$ & AAA & 7.03 \\
\hline 631 & $203318.14+411101.1$ & $20331817+4111010$ & 0.43 & $16.56 \pm 0.13$ & $15.04 \pm 0.08$ & $14.74 \pm 0.12$ & BAB & 11.38 \\
\hline 632 & $203318.17+410920.5$ & $20331821+4109201$ & 0.77 & $15.76 \pm 0.07$ & $14.52 \pm 0.05$ & $14.03 \pm 0.07$ & AAA & 4.11 \\
\hline 633 & $18.28+411739.0$ & $20331828+4$ & 0.13 & \pm 0.02 & $10.97 \pm$ & $10.66 \pm$ & AAA & 6.68 \\
\hline 634 & $203318.30+412209.5$ & $20331837+4122106$ & 1.30 & $15.47 \pm 0.09$ & 11.27 & 10.50 & AUU & 20.34 \\
\hline 635 & $203318.42+411240.4$ & - & - & - & - & - & - & - \\
\hline 636 & $203318.46+411452.9$ & $20331846+4114533$ & 0.17 & $14.91 \pm 0.03$ & $13.69 \pm 0.02$ & $13.22 \pm 0.03$ & AAA & 2.03 \\
\hline 637 & $203318.46+411119.2$ & $20331848+4111195$ & 0.26 & $15.52 \pm 0.06$ & $14.21 \pm 0.04$ & $13.67 \pm 0.05$ & AAA & N/A \\
\hline 638 & $203318.52+411534.9$ & $20331853+4115351$ & 0.11 & $9.41 \pm 0.02$ & $8.58 \pm 0.02$ & $7.98 \pm 0.01$ & AAA & 2.80 \\
\hline 639 & $203318.66+411912.0$ & $20331862+4119122$ & 0.77 & $16.76 \pm 0.17$ & $15.30 \pm 0.10$ & 15.15 & CAU & 8.05 \\
\hline 640 & $203318.66+411723.6$ & $20331865+4117243$ & 0.61 & $17.01 \pm 0.27$ & $15.11 \pm 0.09$ & $14.61 \pm 0.12$ & DAB & N/A \\
\hline 641 & $203318.74+411704.1$ & $20331872+4117044$ & 0.45 & $16.28 \pm 0.19$ & 14.64 & 14.19 & CUU & 1.23 \\
\hline 642 & $203318.77+412130.5$ & $20331873+4121313$ & 1.00 & $15.19 \pm 0.07$ & $14.13 \pm 0.07$ & $13.71 \pm 0.06$ & AAA & 3.27 \\
\hline 643 & $203318.87+411436.8$ & $20331888+4114371$ & 0.15 & $14.97 \pm 0.05$ & $13.17 \pm 0.03$ & $12.01 \pm 0.02$ & AAA & N/A \\
\hline 644 & $203318.91+411319.2$ & - & - & - & - & - & - & - \\
\hline 645 & $203318.94+411332.6$ & $20331892+4113328$ & 0.55 & 15.92 & $14.68 \pm 0.05$ & $14.15 \pm 0.08$ & UAA & 1.12 \\
\hline 646 & $203319.01+412134.8$ & $20331906+4121350$ & 0.66 & $14.17 \pm 0.04$ & $13.12 \pm 0.03$ & $12.66 \pm 0.03$ & AAA & 2.27 \\
\hline 647 & $203318.99+410759.1$ & $20331903+4107589$ & 0.68 & $15.85 \pm 0.07$ & $14.51 \pm 0.07$ & $14.18 \pm 0.08$ & AAA & 7.41 \\
\hline 648 & $203319.06+411354.1$ & $20331909+4113542$ & 0.35 & $15.74 \pm 0.06$ & $14.45 \pm 0.11$ & $13.95 \pm 0.07$ & AAA & N/A \\
\hline 649 & $203319.11+410658.4$ & $20331907+4106585$ & 0.68 & $15.65 \pm 0.06$ & $14.41 \pm 0.05$ & $13.93 \pm 0.07$ & AAA & 0.31 \\
\hline 650 & $203319.13+411659.2$ & $20331914+4116598$ & 0.33 & $14.03 \pm 0.04$ & $13.34 \pm 0.05$ & $13.09 \pm 0.05$ & AEA & 6.80 \\
\hline
\end{tabular}


Table 2. continued.

\begin{tabular}{|c|c|c|c|c|c|c|c|c|}
\hline \multirow{2}{*}{$\begin{array}{l}N_{\mathrm{x}} \\
\#\end{array}$} & \multicolumn{2}{|c|}{ X-ray - 2MASS counterpart } & \multirow{2}{*}{$\begin{array}{l}\text { Off. } \\
\left({ }^{\prime \prime}\right)\end{array}$} & \multicolumn{4}{|c|}{ 2MASS photometry } & \multirow{2}{*}{$\begin{array}{l}\begin{array}{l}A_{\mathrm{v}} \\
(\mathrm{mag})\end{array} \\
\text { (a) }\end{array}$} \\
\hline & CXOAC J+ & 2MASS J+ & & $J$ & $\bar{H}$ & $K_{\mathrm{s}}$ & Ph. qual & \\
\hline 651 & $203319.16+411906.7$ & - & - & - & - & - & - & - \\
\hline 652 & $203319.21+411756.4$ & $20331924+4117567$ & 0.23 & $14.84 \pm 0.02$ & $13.79 \pm 0.02$ & $13.30 \pm 0.04$ & AAA & 6.78 \\
\hline 653 & $203319.29+411702.1$ & - & - & - & - & - & - & - \\
\hline 654 & $203319.42+411741.4$ & - & - & - & - & - & - & - \\
\hline 655 & $203319.44+411349.8$ & $20331943+4113495$ & 0.65 & $15.18 \pm 0.08$ & $14.11 \pm 0.07$ & $13.69 \pm 0.06$ & AAA & 9.68 \\
\hline 656 & $203319.46+412008.5$ & $20331945+4120088$ & 0.13 & $16.07 \pm 0.08$ & $14.65 \pm 0.05$ & $14.14 \pm 0.08$ & AAA & 0.26 \\
\hline 657 & $203319.48+411517.6$ & $20331948+4115173$ & 0.49 & $16.13 \pm 0.10$ & 14.32 & 13.66 & AUU & 14.93 \\
\hline 658 & $203319.48+411225.7$ & $20331950+4112259$ & 0.22 & $15.53 \pm 0.06$ & $14.35 \pm 0.06$ & 13.73 & AAU & 2.97 \\
\hline 659 & $203319.48+410737.0$ & $20331948+4107373$ & 0.09 & $12.81 \pm 0.02$ & $12.03 \pm 0.01$ & $11.60 \pm 0.02$ & AAA & 7.49 \\
\hline 660 & $203319.52+412127.0$ & $20331963+4121270$ & 1.44 & $15.11 \pm 0.04$ & $13.79 \pm 0.05$ & $13.23 \pm 0.04$ & AAA & N/A \\
\hline 661 & $203319.58+411906.4$ & $20331959+4119064$ & 0.29 & $11.95 \pm 0.02$ & $11.47 \pm 0.01$ & $11.20 \pm 0.01$ & AAA & 1.31 \\
\hline 662 & $203319.59+411803.9$ & $20331962+4118043$ & 0.29 & 16.05 & 15.01 & $14.17 \pm 0.08$ & UUA & N/A \\
\hline 663 & $203319.59+411042.3$ & $20331959+4110425$ & 0.12 & $14.79 \pm 0.03$ & $13.69 \pm 0.03$ & $13.24 \pm 0.03$ & AAA & 10.94 \\
\hline 664 & $203319.73+412103.1$ & $20331968+4121025$ & 1.32 & $15.61 \pm 0.06$ & $14.40 \pm 0.05$ & $13.99 \pm 0.07$ & AAA & 6.69 \\
\hline 665 & $203319.88+411124.6$ & $20331986+4111250$ & 0.42 & $16.44 \pm 0.12$ & $15.43 \pm 0.10$ & $14.71 \pm 0.11$ & BAB & 6.72 \\
\hline 666 & $203319.91+410716.8$ & $20331982+4107179$ & 1.70 & 13.49 & $14.10 \pm 0.11$ & 11.33 & UBU & 7.62 \\
\hline 667 & $203320.02+412011.6$ & $20332000+4120117$ & 0.47 & $15.72 \pm 0.08$ & $14.46 \pm 0.05$ & $13.95 \pm 0.07$ & AAA & 1.55 \\
\hline 668 & $203320.03+411831.6$ & - & - & - & - & - & - & - \\
\hline 669 & $203320.09+411632.8$ & - & - & - & - & - & - & - \\
\hline 670 & $203320.12+411943.2$ & $20332016+4119432$ & 0.47 & $13.94 \pm 0.02$ & $12.82 \pm 0.02$ & $12.41 \pm 0.02$ & AAA & 4.49 \\
\hline 671 & $203320.19+411716.4$ & - & - & - & - & - & - & - \\
\hline 672 & $203320.23+411432.3$ & $20332024+4114326$ & 0.12 & $15.24 \pm 0.04$ & $14.04 \pm 0.03$ & $13.62 \pm 0.05$ & AAA & 6.21 \\
\hline 673 & $203320.31+411647.1$ & $20332028+4116478$ & 0.68 & $16.51 \pm 0.12$ & $15.19 \pm 0.09$ & $14.53 \pm 0.11$ & BAB & 5.36 \\
\hline 674 & $203320.39+411840.8$ & $20332040+4118412$ & 0.12 & $13.08 \pm 0.02$ & $12.48 \pm 0.02$ & $12.37 \pm 0.02$ & AAA & 3.66 \\
\hline 675 & $203320.40+411929.0$ & - & - & - & - & - & - & - \\
\hline 676 & $203320.47+412033.0$ & $20332044+4120334$ & 0.59 & $12.89 \pm 0.02$ & $12.22 \pm 0.03$ & $12.03 \pm 0.02$ & AAA & 6.95 \\
\hline 677 & $203320.49+412144.8$ & $20332047+4121447$ & 0.56 & $16.15 \pm 0.09$ & $15.15 \pm 0.08$ & $14.62 \pm 0.12$ & $\mathrm{AAB}$ & 5.88 \\
\hline 678 & $203320.55+411815.6$ & - & - & - & - & - & - & - \\
\hline 679 & $203320.58+411451.3$ & $20332058+4114518$ & 0.25 & $3 \pm 0.19$ & $15.16 \pm 0.08$ & $14.13 \pm 0.07$ & CAA & 4.72 \\
\hline 680 & $203320.58+411704.4$ & $20332057+4117048$ & 0.36 & $14.52 \pm 0.03$ & $13.64 \pm 0.03$ & $13.28 \pm 0.04$ & AAA & 8.51 \\
\hline 681 & $203320.58+411653.6$ & $20332057+4116539$ & 0.23 & $16.65 \pm 0.14$ & $14.95 \pm 0.08$ & $14.15 \pm 0.08$ & BAA & N/A \\
\hline 682 & $203320.68+411418.4$ & - & - & - & - & - & - & - \\
\hline 683 & $203320.76+411723.1$ & $20332076+4117235$ & 0.17 & 12.40 & 11.79 & $12.60 \pm 0.03$ & UUA & N/A \\
\hline 684 & $203320.83+411013.4$ & - & - & - & - & - & - & - \\
\hline 685 & $203320.93+411207.9$ & $20332093+4112080$ & 0.15 & 17.34 & 15.83 & $14.81 \pm 0.14$ & UUB & N/A \\
\hline 686 & $203321.01+411739.8$ & $20332101+4117401$ & 0.03 & $9.30 \pm 0.02$ & $8.90 \pm 0.01$ & $8.67 \pm 0.01$ & AAA & 11.12 \\
\hline 687 & $203321.04+411708.8$ & - & - & - & - & - & - & - \\
\hline 688 & $203321.04+410647.3$ & $20332108+4106483$ & 0.88 & $16.19 \pm 0.09$ & $14.82 \pm 0.06$ & $14.42 \pm 0.09$ & AAA & 38.89 \\
\hline 689 & $203321.10+411659.2$ & - & - & - & - & - & - & - \\
\hline 690 & $203321.11+411756.6$ & $20332112+4117570$ & 0.23 & $14.54 \pm 0.03$ & $13.26 \pm 0.05$ & $12.40 \pm 0.04$ & AAA & N/A \\
\hline 691 & $203321.14+411242.1$ & $20332112+4112436$ & 1.37 & $16.18 \pm 0.10$ & $14.85 \pm 0.07$ & $14.56 \pm 0.10$ & AAA & N/A \\
\hline 692 & $203321.15+411325.4$ & $20332116+4113257$ & 0.07 & $13.94 \pm 0.02$ & $13.07 \pm 0.02$ & $12.64 \pm 0.03$ & AAA & 5.92 \\
\hline 693 & $203321.21+411845.9$ & $20332123+4118462$ & 0.23 & $15.88 \pm 0.08$ & $14.77 \pm 0.06$ & $14.50 \pm 0.10$ & AAA & 12.40 \\
\hline 694 & $203321.25+411839.4$ & $20332126+4118395$ & 0.19 & $13.03 \pm 0.02$ & $12.45 \pm 0.02$ & $12.16 \pm 0.02$ & AAA & N/A \\
\hline 695 & $203321.27+411250.3$ & $20332128+4112507$ & 0.17 & 13.38 & 12.58 & $12.13 \pm 0.02$ & UUA & 7.11 \\
\hline 696 & $203321.29+411312.8$ & - & - & - & - & - & - & - \\
\hline 697 & $203321.59+411229.1$ & $20332161+4112296$ & 0.29 & $15.76 \pm 0.07$ & $14.43 \pm 0.06$ & $13.95 \pm 0.06$ & AAA & N/A \\
\hline 698 & $203321.64+411127.0$ & - & - & - & - & - & - & - \\
\hline 699 & $203321.69+412026.3$ & $20332169+4120262$ & 0.34 & $14.98 \pm$ & $14.17 \pm$ & $13.77 \pm$ & AAA & N/A \\
\hline 700 & $203321.73+411511.1$ & - & - & - & - & - & - & - \\
\hline 701 & $203321.81+411332.2$ & $20332182+4113325$ & 0.01 & $12.51 \pm 0.02$ & $11.75 \pm 0.01$ & $11.34 \pm 0.02$ & AAA & N/A \\
\hline 702 & $203321.85+412136.1$ & $20332184+4121367$ & 0.46 & $14.90 \pm 0.04$ & $13.55 \pm 0.03$ & $13.12 \pm 0.04$ & AAA & 11.02 \\
\hline 703 & $203321.83+410944.6$ & $20332184+4109445$ & 0.30 & $15.14 \pm 0.05$ & $13.68 \pm 0.03$ & $12.98 \pm 0.06$ & AAA & N/A \\
\hline 704 & $203321.90+411316.5$ & $20332193+4113167$ & 0.22 & $15.33 \pm 0.04$ & $14.00 \pm 0.03$ & $13.47 \pm 0.04$ & AAA & 7.27 \\
\hline 705 & $203321.92+411731.3$ & $20332194+4117317$ & 0.20 & $15.14 \pm 0.05$ & $14.11 \pm 0.04$ & $13.63 \pm 0.05$ & AAA & 20.23 \\
\hline 706 & $203321.97+412037.2$ & $20332191+4120371$ & 0.93 & $15.94 \pm 0.08$ & $14.65 \pm 0.06$ & $14.23 \pm 0.09$ & AAA & 10.17 \\
\hline 707 & $203322.00+411431.3$ & $20332201+4114314$ & 0.08 & $16.59 \pm 0.14$ & $15.44 \pm 0.12$ & $14.65 \pm 0.12$ & BBB & N/A \\
\hline 708 & $203322.01+411217.5$ & $20332202+4112179$ & 0.17 & $15.02 \pm 0.04$ & $13.76 \pm 0.04$ & $13.29 \pm 0.04$ & AAA & N/A \\
\hline 709 & $203322.06+412153.8$ & - & - & - & - & - & - & - \\
\hline 710 & $203322.06+411003.9$ & $20332206+4110044$ & 0.29 & 16.35 & $15.18 \pm 0.08$ & 14.24 & UAU & 8.69 \\
\hline 711 & $203322.12+411245.1$ & $20332214+4112453$ & 0.11 & $15.40 \pm 0.05$ & $14.11 \pm 0.03$ & $13.64 \pm 0.05$ & AAA & 1.68 \\
\hline 712 & $203322.25+411744.3$ & $20332224+4117446$ & 0.23 & $13.04 \pm 0.02$ & $12.01 \pm 0.03$ & $11.60 \pm 0.02$ & AAA & 7.99 \\
\hline 713 & $203322.34+411815.8$ & - & - & - & - & - & - & - \\
\hline 714 & $203322.37+411551.4$ & $20332236+4115518$ & 0.29 & $16.48 \pm 0.12$ & $14.87 \pm 0.07$ & $14.28 \pm 0.08$ & BAA & 7.63 \\
\hline 715 & $203322.40+411551.4$ & $20332236+4115518$ & 0.70 & $16.48 \pm 0.12$ & $14.87 \pm 0.07$ & $14.28 \pm 0.08$ & BAA & 5.13 \\
\hline
\end{tabular}


Table 2. continued.

\begin{tabular}{|c|c|c|c|c|c|c|c|c|}
\hline \multirow{2}{*}{$\begin{array}{l}N_{\mathrm{x}} \\
\#\end{array}$} & \multicolumn{2}{|c|}{ X-ray - 2MASS counterpart } & \multirow{2}{*}{$\begin{array}{l}\text { Off. } \\
\left({ }^{\prime \prime}\right)\end{array}$} & \multicolumn{4}{|c|}{ 2MASS photometry } & \multirow{2}{*}{$\begin{array}{l}\begin{array}{l}A_{\mathrm{v}} \\
(\mathrm{mag})\end{array} \\
\text { (a) }\end{array}$} \\
\hline & CXOAC J+ & 2MASS J+ & & $J$ & $\bar{H}$ & $\bar{K}_{\mathrm{s}}$ & Ph. qual & \\
\hline 716 & $203322.44+411736.5$ & $20332247+4117368$ & 0.44 & $16.36 \pm 0.12$ & $14.96 \pm 0.09$ & $14.38 \pm 0.11$ & BAA & N/A \\
\hline 717 & $203322.57+411340.2$ & $20332260+4113403$ & 0.37 & $15.62 \pm 0.09$ & $14.44 \pm 0.13$ & $13.88 \pm 0.08$ & ABA & 7.10 \\
\hline 718 & $203322.91+411859.8$ & $20332290+4119002$ & 0.25 & $15.29 \pm 0.04$ & $14.13 \pm 0.06$ & $13.59 \pm 0.05$ & AAA & N/A \\
\hline 719 & $203323.02+411612.0$ & - & - & - & - & - & - & - \\
\hline 720 & $203323.02+410932.5$ & $20332294+4109324$ & 1.25 & $16.42 \pm 0.15$ & $14.81 \pm 0.10$ & $14.23 \pm 0.09$ & BAA & 5.84 \\
\hline 721 & $203323.02+411222.0$ & $20332300+4112218$ & 0.58 & $12.77 \pm 0.02$ & $12.01 \pm 0.02$ & $11.82 \pm 0.02$ & AAA & N/A \\
\hline 722 & $203323.02+411222.1$ & $20332300+4112218$ & 0.58 & $12.77 \pm 0.02$ & $12.01 \pm 0.02$ & $11.82 \pm 0.02$ & AAA & 9.35 \\
\hline 723 & $203323.22+411909.2$ & - & - & - & - & - & - & - \\
\hline 724 & $203323.34+411236.2$ & $20332335+4112364$ & 0.05 & $14.73 \pm 0.04$ & $13.52 \pm 0.04$ & $13.01 \pm 0.03$ & AAA & 12.66 \\
\hline 725 & $203323.38+411912.1$ & $20332336+4119126$ & 0.51 & $14.81 \pm 0.04$ & $13.75 \pm 0.04$ & $13.35 \pm 0.04$ & AAA & 10.53 \\
\hline 726 & $203323.37+411347.3$ & $20332337+4113478$ & 0.21 & 13.09 & $13.99 \pm 0.12$ & $13.67 \pm 0.07$ & UBA & 5.51 \\
\hline 727 & $203323.38+411747.8$ & - & - & - & - & - & - & - \\
\hline 728 & $203323.43+412039.8$ & $20332345+4120398$ & 0.29 & $13.84 \pm 0.02$ & $13.23 \pm 0.02$ & $12.88 \pm 0.03$ & AAA & 7.59 \\
\hline 729 & $203323.48+410912.2$ & $20332346+4109130$ & 0.68 & $7.03 \pm 0.01$ & $6.38 \pm 0.01$ & $6.05 \pm 0.01$ & AAA & 0.97 \\
\hline 730 & $203323.57+411746.8$ & $20332354+4117475$ & 0.84 & 15.12 & $14.14 \pm 0.06$ & $13.65 \pm 0.05$ & UAA & N/A \\
\hline 731 & $203323.67+412151.0$ & $20332364+4121511$ & 0.67 & $12.93 \pm 0.02$ & $12.04 \pm 0.02$ & $11.64 \pm 0.02$ & AAA & 8.08 \\
\hline 732 & $203323.73+411537.3$ & $20332377+4115375$ & 0.44 & $15.47 \pm 0.08$ & $14.27 \pm 0.03$ & $13.59 \pm 0.07$ & AAA & 14.46 \\
\hline 733 & $203323.77+412006.4$ & $20332378+4120068$ & 0.13 & $14.97 \pm 0.05$ & $13.84 \pm 0.04$ & $13.34 \pm 0.04$ & AAA & 2.24 \\
\hline 734 & $203323.84+411310.6$ & $20332384+4113111$ & 0.27 & $14.34 \pm 0.03$ & $13.14 \pm 0.03$ & $12.69 \pm 0.03$ & AAA & 0.01 \\
\hline 735 & $203323.83+411106.4$ & - & - & - & - & - & - & - \\
\hline 736 & $203323.89+412207.8$ & $20332391+4122083$ & 0.31 & $15.43 \pm 0.06$ & $14.24 \pm 0.05$ & $13.88 \pm 0.06$ & AAA & 12.64 \\
\hline 737 & $203323.94+411640.2$ & $20332398+4116405$ & 0.45 & $15.65 \pm 0.06$ & $14.40 \pm 0.06$ & $13.94 \pm 0.07$ & AAA & 1.31 \\
\hline 738 & $203324.01+412145.9$ & $20332409+4121449$ & 1.59 & $16.17 \pm 0.11$ & $14.98 \pm 0.08$ & $14.62 \pm 0.13$ & BAB & N/A \\
\hline 739 & $203324.25+411700.1$ & $20332426+4117004$ & 0.13 & $14.45 \pm 0.03$ & $13.33 \pm 0.02$ & $12.88 \pm 0.03$ & AAA & 11.61 \\
\hline 740 & $203324.31+412106.7$ & - & - & - & - & - & - & - \\
\hline 741 & $203324.32+411752.5$ & $20332434+4117515$ & 1.21 & $11.00 \pm 0.02$ & $10.39 \pm 0.02$ & $10.27 \pm 0.01$ & AAA & 6.91 \\
\hline 742 & $203324.38+411416.6$ & $20332442+4114164$ & 0.62 & $16.65 \pm 0.15$ & $15.49 \pm 0.10$ & $15.11 \pm 0.17$ & BAC & N/A \\
\hline 743 & $203324.41+412032.8$ & $20332440+4120330$ & 0.12 & $15.53 \pm 0.06$ & $14.18 \pm 0.07$ & $13.80 \pm 0.07$ & AAA & 8.84 \\
\hline 744 & $203324.39+410812.3$ & $20332439+4108131$ & 0.62 & $15.65 \pm 0.05$ & $14.19 \pm 0.04$ & $13.69 \pm 0.05$ & AAA & N/A \\
\hline 745 & $203324.48+411532.7$ & $20332453+4115339$ & 1.16 & $13.31 \pm 0.02$ & $12.17 \pm 0.02$ & $11.70 \pm 0.02$ & AAA & 10.25 \\
\hline 746 & $203324.53+411534.0$ & $20332453+4115339$ & 0.29 & $13.31 \pm 0.02$ & $12.17 \pm 0.02$ & $11.70 \pm 0.02$ & AAA & N/A \\
\hline 747 & $203324.55+411046.3$ & $20332461+4110451$ & 1.60 & 16.86 & $15.80 \pm 0.14$ & 14.67 & UBU & 6.52 \\
\hline 748 & $203324.58+411907.9$ & $20332463+$ & 0.54 & 05 & $14.26 \pm 0.04$ & 14 & AAU & N/A \\
\hline 749 & $203324.60+411553.8$ & $20332461+4115542$ & 0.17 & $2 \pm 0.13$ & $15.22 \pm 0.08$ & $14.55 \pm$ & BAB & N/A \\
\hline 750 & $203324.62+411059.4$ & $20332464+4110596$ & 0.22 & $16.02 \pm 0.08$ & $14.72 \pm 0.05$ & $14.32 \pm 0.09$ & AAA & 15.96 \\
\hline 751 & $203324.77+412223.1$ & $20332481+4122235$ & 0.59 & $15.32 \pm 0.05$ & $14.19 \pm 0.04$ & $13.70 \pm$ & AAA & 3.27 \\
\hline 752 & $203324.89+412001.6$ & - & - & - & - & - & - & - \\
\hline 753 & $203324.96+410836.3$ & $20332497+4108366$ & 0.12 & $16.37 \pm 0.17$ & 14.30 & 13.80 & CUU & 3.90 \\
\hline 754 & $203325.01+411537.0$ & $20332503+4115375$ & 0.22 & $16.06 \pm 0.10$ & $14.57 \pm 0.06$ & $14.05 \pm 0.07$ & AAA & N/A \\
\hline 755 & $203325.12+411818.6$ & $20332513+4118187$ & 0.17 & $13.20 \pm 0.02$ & $12.71 \pm 0.02$ & $12.43 \pm 0.02$ & AAA & N/A \\
\hline 756 & $203325.10+411050.1$ & $20332509+4110515$ & 1.17 & $16.71 \pm 0.15$ & $15.18 \pm 0.08$ & $15.04 \pm 0.15$ & BAC & 8.01 \\
\hline 757 & $203325.10+410724.8$ & $20332511+4107248$ & 0.25 & $15.98 \pm 0.08$ & $14.62 \pm 0.05$ & $13.97 \pm 0.06$ & AAA & 0.89 \\
\hline 758 & $203325.23+411146.1$ & - & - & - & - & - & - & - \\
\hline 759 & $203325.27+410918.4$ & - & - & - & - & - & - & - \\
\hline 760 & $203325.42+411039.4$ & $20332541+4110400$ & 0.41 & $14.92 \pm 0.05$ & $13.57 \pm 0.05$ & $13.18 \pm 0.04$ & AAA & 11.96 \\
\hline 761 & $203325.50+410932.1$ & $20332548+4109314$ & 1.05 & $15.74 \pm 0.06$ & $0 \pm 0.04$ & $13.74 \pm 0.05$ & AAA & N/A \\
\hline 762 & $203325.66+411555.6$ & $20332563+4$ & 0.58 & \pm 0.16 & $15.17 \pm 0.10$ & $4 \pm 0.11$ & CAA & N/A \\
\hline 763 & $5.82+411853.6$ & $20332584+$ & 0.33 & \pm 0.06 & $14.00 \pm 0.05$ & $13.46 \pm$ & AAA & N/A \\
\hline 764 & $203325.91+411322.2$ & $20332594+4$ & 0.50 & $15.02 \pm 0.04$ & $13.79 \pm 0.04$ & $13.46 \pm$ & AAA & N/A \\
\hline 765 & $203325.92+411333.2$ & $20332592+4113332$ & 0.17 & $15.86 \pm 0.08$ & $14.40 \pm 0.05$ & $13.70 \pm 0.06$ & AAA & 6.69 \\
\hline 766 & $203325.93+411718.6$ & $20332597+4117191$ & 0.52 & 17.07 & $15.30 \pm 0.09$ & $15.14 \pm 0.19$ & UAC & 8.57 \\
\hline 767 & $203325.94+411316.5$ & - & - & - & - & - & - & - \\
\hline 768 & $203326.03+411901.1$ & $20332601+4$ & 0.56 & $16.57 \pm 0.16$ & $14.99 \pm 0.10$ & $14.58 \pm 0.12$ & $\mathrm{CAB}$ & N/A \\
\hline 769 & $203326.08+411229.6$ & $20332611+4112298$ & 0.33 & $13.74 \pm 0.02$ & $12.74 \pm 0.02$ & $12.32 \pm 0.02$ & AAA & N/A \\
\hline 770 & $203326.11+411549.0$ & $20332608+4115497$ & 0.76 & $11.66 \pm 0.02$ & $10.98 \pm 0.02$ & $10.62 \pm 0.02$ & AAA & 5.73 \\
\hline 771 & $203326.28+412146.7$ & - & - & - & - & - & - & - \\
\hline 772 & $203326.33+411806.0$ & $20332632+4118059$ & 0.39 & $15.97 \pm 0.07$ & $14.67 \pm 0.06$ & $14.18 \pm 0.08$ & AAA & 11.84 \\
\hline 773 & $203326.33+411238.4$ & $20332633+4112388$ & 0.24 & $14.42 \pm 0.03$ & $13.38 \pm 0.03$ & $12.91 \pm 0.03$ & AAA & 8.89 \\
\hline 774 & $203326.33+410819.1$ & $20332629+4108174$ & 1.97 & $15.51 \pm 0.05$ & $14.90 \pm 0.07$ & $14.83 \pm 0.14$ & AAB & 10.07 \\
\hline 775 & $203326.44+411048.5$ & - & - & - & - & - & - & - \\
\hline 776 & $203326.55+411503.6$ & $20332654+4115041$ & 0.45 & $16.18 \pm 0.09$ & $14.60 \pm 0.05$ & $13.46 \pm 0.04$ & AAA & 3.96 \\
\hline 777 & $203326.60+411547.3$ & $20332661+4115475$ & 0.12 & $14.05 \pm 0.04$ & $12.89 \pm 0.03$ & $12.40 \pm 0.02$ & AAA & 5.47 \\
\hline 778 & $203326.68+411555.4$ & $20332665+4115560$ & 0.69 & $16.61 \pm 0.14$ & $15.22 \pm 0.12$ & $14.72 \pm 0.13$ & BBB & N/A \\
\hline 779 & $203326.74+411059.3$ & $20332674+4110595$ & 0.11 & $8.97 \pm 0.02$ & $8.43 \pm 0.01$ & $8.16 \pm 0.01$ & AAA & 9.77 \\
\hline 780 & $203326.81+412046.9$ & $20332679+4120471$ & 0.33 & $15.75 \pm 0.07$ & $14.64 \pm 0.06$ & $14.50 \pm 0.11$ & AAB & N/A \\
\hline
\end{tabular}


Table 2. continued.

\begin{tabular}{|c|c|c|c|c|c|c|c|c|}
\hline \multirow{2}{*}{$\begin{array}{l}N_{\mathrm{x}} \\
\#\end{array}$} & \multicolumn{2}{|c|}{ X-ray - 2MASS counterpart } & \multirow{2}{*}{$\begin{array}{l}\text { Off. } \\
\left({ }^{\prime \prime}\right)\end{array}$} & \multicolumn{4}{|c|}{ 2MASS photometry } & \multirow{2}{*}{$\begin{array}{l}\begin{array}{l}A_{\mathrm{v}} \\
(\mathrm{mag})\end{array} \\
\text { (a) }\end{array}$} \\
\hline & CXOAC J+ & 2MASS J+ & & $J$ & $H$ & $K_{\mathrm{s}}$ & Ph. qual & \\
\hline 781 & $203326.79+411217.6$ & - & - & - & - & - & - & - \\
\hline 782 & $203326.85+411741.3$ & $20332686+4117415$ & 0.11 & $15.16 \pm 0.04$ & $13.95 \pm 0.03$ & $13.40 \pm 0.04$ & AAA & 18.25 \\
\hline 783 & $203326.87+411319.1$ & $20332685+4113199$ & 0.65 & $16.62 \pm 0.14$ & $14.93 \pm 0.07$ & $14.25 \pm 0.08$ & BAA & 5.25 \\
\hline 784 & $203327.11+411822.5$ & $20332713+4118225$ & 0.24 & $14.17 \pm 0.02$ & $13.09 \pm 0.02$ & $12.63 \pm 0.02$ & AAA & N/A \\
\hline 785 & $203327.19+411709.8$ & $20332723+4117106$ & 0.71 & $13.02 \pm 0.02$ & $12.29 \pm 0.03$ & $11.91 \pm 0.02$ & AAA & 8.41 \\
\hline 786 & $203327.23+412008.8$ & $20332724+4120088$ & 0.23 & $13.97 \pm 0.03$ & $13.00 \pm 0.03$ & $12.59 \pm 0.03$ & AAA & 9.71 \\
\hline 787 & $203327.30+411525.6$ & $20332733+4115254$ & 0.55 & $16.63 \pm 0.16$ & $15.11 \pm 0.10$ & $14.43 \pm 0.12$ & $\mathrm{CAB}$ & 5.61 \\
\hline 788 & $203327.31+410751.3$ & $20332731+4107534$ & 1.88 & $16.63 \pm 0.14$ & $15.33 \pm 0.10$ & $15.05 \pm 0.16$ & BAC & 8.57 \\
\hline 789 & $203327.45+411626.5$ & $20332742+4116263$ & 0.68 & $16.65 \pm 0.14$ & $15.40 \pm 0.10$ & 14.96 & BAU & N/A \\
\hline 790 & $203327.59+411507.0$ & $20332761+4115064$ & 0.86 & $16.23 \pm 0.13$ & $14.64 \pm 0.08$ & $13.82 \pm 0.06$ & BAA & N/A \\
\hline 791 & $203327.61+412015.7$ & $20332754+4120158$ & 1.22 & 14.37 & $13.47 \pm 0.04$ & 13.04 & UAU & 0.67 \\
\hline 792 & $203327.91+411509.4$ & $20332793+4115097$ & 0.12 & $15.89 \pm 0.07$ & $14.84 \pm 0.07$ & $14.31 \pm 0.09$ & AAA & 7.13 \\
\hline 793 & $203328.32+412126.7$ & $20332828+4121276$ & 0.95 & 16.55 & $15.14 \pm 0.08$ & 14.64 & UAU & N/A \\
\hline 794 & $203328.32+410933.9$ & $20332833+4109341$ & 0.11 & $14.18 \pm 0.03$ & $12.81 \pm 0.03$ & $12.14 \pm 0.02$ & AAA & N/A \\
\hline 795 & $203328.36+411501.3$ & $20332836+4115015$ & 0.23 & $15.71 \pm 0.06$ & $14.43 \pm 0.04$ & $13.85 \pm 0.06$ & AAA & 7.30 \\
\hline 796 & $203328.43+411937.1$ & $20332845+4119373$ & 0.23 & $14.00 \pm 0.03$ & $12.96 \pm 0.02$ & $12.59 \pm 0.02$ & AAA & 13.34 \\
\hline 797 & $203328.49+411915.4$ & $20332851+4119152$ & 0.43 & $15.19 \pm 0.05$ & $14.04 \pm 0.03$ & $13.47 \pm 0.05$ & AAA & 8.11 \\
\hline 798 & $203328.54+410716.7$ & $20332853+4107172$ & 0.34 & 05 & $13.94 \pm 0.04$ & $13.48 \pm 0.04$ & AAA & N/A \\
\hline 799 & $203328.58+411409.4$ & $20332848+4114097$ & 1.76 & $14.64 \pm 0.04$ & $12.80 \pm 0.03$ & $12.01=$ & AAA & 11.67 \\
\hline 800 & $203328.74+411611.3$ & - & - & - & - & - & - & - \\
\hline 801 & $203328.74+411424.0$ & $20332876+4114240$ & 0.28 & $15.04 \pm 0.05$ & $13.65 \pm 0.05$ & $13.22 \pm 0.04$ & AAA & 4.58 \\
\hline 802 & $203329.20+411425.8$ & $20332917+4114263$ & 0.65 & $15.19 \pm 0.05$ & $14.14 \pm 0.05$ & $13.75 \pm 0.06$ & AAA & 3.28 \\
\hline 803 & $203329.26+412138.2$ & $20332922+4121387$ & 0.81 & $15.63 \pm 0.06$ & $14.56 \pm 0.06$ & $14.03 \pm 0.07$ & AAA & N/A \\
\hline 804 & $203329.31+411605.8$ & $20332933+4116058$ & 0.31 & $16.64 \pm 0.14$ & $15.38 \pm 0.10$ & 14.38 & BAU & N/A \\
\hline 805 & $203329.41+411806.2$ & $20332944+4118062$ & 0.39 & $17.06 \pm 0.22$ & $15.52 \pm 0.11$ & 15.81 & $\mathrm{CBU}$ & 5.57 \\
\hline 806 & $203329.45+412034.8$ & $20332948+4120344$ & 0.68 & $13.07 \pm 0.02$ & $12.61 \pm 0.02$ & $12.32 \pm 0.02$ & AAA & 6.68 \\
\hline 807 & $203329.48+411858.7$ & $20332950+4118589$ & 0.12 & $15.90 \pm 0.07$ & $14.64 \pm 0.07$ & $14.13 \pm 0.08$ & AAA & 15.80 \\
\hline 808 & $203329.57+412005.5$ & $20332962+4120060$ & 0.75 & $14.57 \pm 0.05$ & $13.36 \pm 0.03$ & $12.85 \pm 0.03$ & AAA & N/A \\
\hline 809 & $203329.72+411557.2$ & $20332973+4115577$ & 0.24 & $14.10 \pm 0.04$ & $12.98 \pm 0.03$ & $12.54 \pm 0.03$ & AAA & N/A \\
\hline 810 & $203329.95+411218.5$ & $20332994+4112185$ & 0.39 & $15.65 \pm 0.06$ & $14.60 \pm 0.06$ & $14.17 \pm 0.07$ & AAA & 7.13 \\
\hline 811 & $203330.25+411608.5$ & $20333027+4116087$ & 0.22 & 05 & $14.00 \pm 0.04$ & $13.51 \pm 0.04$ & AAA & N/A \\
\hline 812 & $203330.32+411406.2$ & $20333036+4114064$ & 0.34 & 02 & $12.97 \pm 0.02$ & $12.49 \pm 0.03$ & AAA & N/A \\
\hline 813 & $30.47+412016.4$ & $20333052+$ & 0.68 & 02 & $10.53 \pm 0.01$ & 0.01 & AAA & 6.90 \\
\hline 814 & $203330.47+410935.7$ & $20333043+4109368$ & 1.19 & \pm & $13.86 \pm 0.04$ & $13.44 \pm$ & AAA & 7.24 \\
\hline 815 & $203330.51+411722.0$ & $20333053+4117219$ & 0.37 & $4 \pm$ & $13.89 \pm 0.03$ & $13.31 \pm$ & AAA & 12.21 \\
\hline 816 & $203330.57+411012.0$ & $20333061+4110119$ & 0.53 & $15.27 \pm 0.04$ & $14.03 \pm 0.04$ & $13.49 \pm 0.04$ & AAA & N/A \\
\hline 817 & $203330.74+411522.7$ & $20333078+4115226$ & 0.56 & $6.49 \pm 0.02$ & $5.89 \pm 0.01$ & $5.54 \pm 0.01$ & AAA & N/A \\
\hline 818 & $203331.06+412025.5$ & $20333104+4120258$ & 0.45 & $15.09 \pm 0.05$ & $14.01 \pm 0.04$ & $13.56 \pm 0.05$ & AAA & N/A \\
\hline 819 & $203331.14+411317.4$ & $20333108+4113180$ & 0.98 & 15.75 & $15.49 \pm 0.11$ & 14.25 & UAU & N/A \\
\hline 820 & $203331.20+411610.4$ & $20333122+4116105$ & 0.25 & $14.97 \pm 0.05$ & $14.07 \pm 0.07$ & $13.63 \pm 0.05$ & AAA & 17.86 \\
\hline 821 & $203331.27+412131.4$ & $20333147+4121311$ & 2.90 & $15.19 \pm 0.05$ & $14.16 \pm 0.04$ & $13.66 \pm 0.05$ & AAA & 8.24 \\
\hline 822 & $203331.33+411602.8$ & $20333136+4116028$ & 0.54 & $15.89 \pm 0.08$ & $14.73 \pm 0.06$ & $14.31 \pm 0.09$ & AAA & 8.51 \\
\hline 823 & $203331.36+411609.6$ & - & - & - & - & - & - & - \\
\hline 824 & $203331.36+411529.3$ & - & - & - & - & - & - & - \\
\hline 825 & $203331.35+410912.5$ & $20333137+4109123$ & 0.55 & $15.21 \pm 0.05$ & $14.12 \pm 0.04$ & $13.64 \pm 0.05$ & AAA & 15.63 \\
\hline 826 & $203331.35+410912.6$ & $20333137+4109123$ & 0.55 & $1 \pm 0.05$ & $14.12 \pm 0.04$ & $13.64 \pm 0.05$ & AAA & N/A \\
\hline 827 & $203331.72+411532.9$ & $20333169+411$ & 0.80 & \pm 0.11 & $5 \pm 0.10$ & $13.78 \pm 0.07$ & AAA & N/A \\
\hline 828 & $203331.77+412047.2$ & 2033 & 0.17 & 03 & $12.36 \pm 0.04$ & 12. & AAA & 8.16 \\
\hline 829 & $203331.87+411003.3$ & $20333190+4110041$ & 0.62 & \pm 0.08 & $14.60 \pm 0.05$ & $14.11 \pm$ & AAA & 14.06 \\
\hline 830 & $203331.91+411642.9$ & $20333194+4116432$ & 0.45 & 15.30 & $14.17 \pm 0.08$ & 13.11 & UAU & 6.22 \\
\hline 831 & $203332.11+412122.2$ & $20333215+4121233$ & 1.02 & $12.94 \pm 0.02$ & $12.28 \pm 0.02$ & $11.95 \pm 0.02$ & AAA & N/A \\
\hline 832 & $203332.11+412019.7$ & - & - & - & - & - & - & - \\
\hline 833 & $203332.05+410558.7$ & - & - & - & - & - & - & - \\
\hline 834 & $203332.16+411801.9$ & $20333215+4118023$ & 0.28 & 16. & 15.69 & $14.95 \pm 0.16$ & UUC & N/A \\
\hline 835 & $203332.28+411639.2$ & $20333229+4116397$ & 0.27 & 15.40 & $14.50 \pm 0.06$ & 13.39 & UAU & 0.08 \\
\hline 836 & $203332.38+411155.8$ & $20333229+4111566$ & 1.56 & 15.36 & $14.64 \pm 0.08$ & $14.01 \pm 0.08$ & UAA & 24.08 \\
\hline 837 & $203332.47+411543.1$ & $20333248+4115434$ & 0.06 & $15.19 \pm 0.04$ & $13.98 \pm 0.03$ & $13.37 \pm 0.04$ & AAA & 4.33 \\
\hline 838 & $203332.68+411100.1$ & $20333265+4110596$ & 0.89 & $15.82 \pm 0.07$ & 14.22 & 13.73 & AUU & N/A \\
\hline 839 & $203332.74+411251.8$ & $20333271+4112522$ & 0.58 & $16.26 \pm 0.09$ & $14.74 \pm 0.06$ & $13.97 \pm 0.07$ & AAA & N/A \\
\hline 840 & $203332.81+410519.6$ & $20333278+4105202$ & 0.58 & $12.93 \pm 0.03$ & $12.16 \pm 0.02$ & $11.73 \pm 0.02$ & EAA & N/A \\
\hline 841 & $203332.92+412147.2$ & - & - & - & - & - & - & - \\
\hline 842 & $203332.93+411040.4$ & $20333293+4110405$ & 0.24 & $15.70 \pm 0.06$ & $14.42 \pm 0.05$ & $13.91 \pm$ & AAA & 7.27 \\
\hline 843 & $203333.01+411428.5$ & $20333304+4114287$ & 0.22 & $14.68 \pm 0.04$ & $13.56 \pm 0.03$ & $13.09 \pm 0.04$ & AAA & N/A \\
\hline 844 & $203333.09+411730.0$ & $20333308+4117298$ & 0.46 & $15.90 \pm 0.07$ & $14.61 \pm 0.05$ & $14.27 \pm 0.08$ & AAA & 0.20 \\
\hline 845 & $203333.09+411132.4$ & $20333294+4111321$ & 2.36 & $15.33 \pm 0.06$ & $14.30 \pm 0.06$ & $13.78 \pm 0.06$ & AAA & 8.03 \\
\hline
\end{tabular}


Table 2. continued.

\begin{tabular}{|c|c|c|c|c|c|c|c|c|}
\hline \multirow{2}{*}{$\begin{array}{l}N_{\mathrm{x}} \\
\#\end{array}$} & \multicolumn{2}{|c|}{ X-ray - 2MASS counterpart } & \multirow{2}{*}{$\begin{array}{l}\text { Off. } \\
\left({ }^{\prime \prime}\right)\end{array}$} & \multicolumn{4}{|c|}{ 2MASS photometry } & \multirow{2}{*}{$\begin{array}{l}\begin{array}{l}A_{\mathrm{v}} \\
(\mathrm{mag})\end{array} \\
\text { (a) }\end{array}$} \\
\hline & CXOAC J+ & 2MASS J+ & & $J$ & $H$ & $K_{\mathrm{s}}$ & Ph.qual & \\
\hline 846 & $203333.43+411917.2$ & $20333351+4119166$ & 1.41 & $15.38 \pm 0.05$ & $14.52 \pm 0.05$ & $14.02 \pm 0.07$ & AAA & 11.25 \\
\hline 847 & $203333.44+410803.1$ & $20333343+4108031$ & 0.31 & $15.50 \pm 0.05$ & $14.12 \pm 0.04$ & $13.73 \pm 0.05$ & AAA & 7.78 \\
\hline 848 & $203333.72+412152.1$ & $20333357+4121521$ & 2.43 & $11.94 \pm 0.02$ & $11.45 \pm 0.02$ & $11.19 \pm 0.01$ & AAA & 5.48 \\
\hline 849 & $203333.78+411937.0$ & $20333393+4119382$ & 2.40 & $10.27 \pm 0.02$ & $9.66 \pm 0.01$ & $9.42 \pm 0.01$ & $\mathrm{AEE}$ & 10.14 \\
\hline 850 & $203333.96+411129.5$ & $20333399+4111292$ & 0.61 & $16.49 \pm 0.11$ & $14.99 \pm 0.07$ & $14.67 \pm 0.12$ & BAB & N/A \\
\hline 851 & $203334.06+412145.9$ & - & - & - & - & - & - & - \\
\hline 852 & $203334.26+411851.4$ & $20333427+4118520$ & 0.36 & 15.90 & $15.00 \pm 0.12$ & 13.60 & UBU & 7.30 \\
\hline 853 & $203334.27+411422.2$ & $20333429+4114222$ & 0.30 & $15.97 \pm 0.07$ & $14.84 \pm 0.06$ & $14.33 \pm 0.09$ & AAA & N/A \\
\hline 854 & $203334.53+412136.6$ & $20333456+4121372$ & 0.53 & $11.59 \pm 0.02$ & $11.18 \pm 0.01$ & $10.93 \pm 0.01$ & AAA & 1.31 \\
\hline 855 & $203334.55+411622.2$ & - & - & - & - & - & - & - \\
\hline 856 & $203334.62+412039.6$ & $20333479+4120382$ & 2.92 & $15.27 \pm 0.05$ & $14.08 \pm 0.04$ & $13.66 \pm 0.05$ & AAA & 13.89 \\
\hline 857 & $203334.74+412050.5$ & $20333478+4120502$ & 0.77 & 16.62 & $15.65 \pm 0.12$ & $14.92 \pm 0.15$ & UBB & N/A \\
\hline 858 & $203334.74+411147.7$ & $20333474+4111481$ & 0.15 & $15.64 \pm 0.05$ & $14.64 \pm 0.05$ & $14.13 \pm 0.07$ & AAA & 6.78 \\
\hline 859 & $203334.79+411456.1$ & $20333471+4114574$ & 1.79 & $15.93 \pm 0.08$ & $14.85 \pm 0.06$ & $14.13 \pm 0.08$ & AAA & N/A \\
\hline 860 & $203334.80+410748.7$ & $20333481+4107473$ & 1.68 & $15.99 \pm 0.08$ & $14.84 \pm 0.07$ & $14.27 \pm 0.09$ & AAA & 5.73 \\
\hline 861 & $203334.92+410857.5$ & $20333494+4108577$ & 0.11 & $13.75 \pm 0.02$ & $13.14 \pm 0.02$ & $12.82 \pm 0.03$ & AAA & 7.76 \\
\hline 862 & $203335.05+411612.2$ & - & - & - & - & - & - & - \\
\hline 863 & $203335.22+411119.2$ & $20333523+4111195$ & 0.11 & $15.45 \pm 0.07$ & $14.19 \pm 0.08$ & $13.69=$ & AAA & 7.81 \\
\hline 864 & $203335.33+412059.5$ & - & - & - & - & - & - & - \\
\hline 865 & $203335.33+411821.8$ & $20333535+4118221$ & 0.24 & $16.11 \pm 0.10$ & $15.05 \pm 0.09$ & $14.64 \pm 0.12$ & $\mathrm{AAB}$ & 6.17 \\
\hline 866 & $203335.28+410714.0$ & - & - & - & - & - & - & - \\
\hline 867 & $203335.57+411659.0$ & $20333541+4116584$ & 2.69 & $12.94 \pm 0.02$ & $12.24 \pm 0.02$ & $11.88 \pm 0.02$ & AAA & N/A \\
\hline 868 & $203335.61+411556.4$ & - & - & - & - & - & - & - \\
\hline 869 & $203335.66+411205.9$ & $20333572+4112068$ & 1.13 & $16.76 \pm 0.16$ & $15.58 \pm 0.13$ & $15.19 \pm 0.18$ & $\mathrm{CBC}$ & 7.11 \\
\hline 870 & $203335.66+410630.5$ & $20333565+4106303$ & 0.54 & $15.34 \pm 0.06$ & $14.21 \pm 0.05$ & $13.67 \pm 0.05$ & AAA & 7.18 \\
\hline 871 & $203335.80+411519.5$ & $20333584+4115192$ & 0.67 & 16.35 & $15.60 \pm 0.14$ & 14.39 & UBU & 5.55 \\
\hline 872 & $203335.87+410649.5$ & $20333585+4106506$ & 0.97 & $15.78 \pm 0.07$ & $14.57 \pm 0.05$ & $14.23 \pm 0.08$ & AAA & 33.68 \\
\hline 873 & $203336.00+411834.5$ & $20333602+4118344$ & 0.29 & $15.93 \pm 0.08$ & $14.55 \pm 0.05$ & $14.14 \pm 0.08$ & AAA & N/A \\
\hline 874 & $203336.25+410812.7$ & $20333626+4108116$ & 1.37 & $13.98 \pm 0.03$ & $12.79 \pm 0.03$ & $12.40 \pm 0.03$ & AAA & 7.59 \\
\hline 875 & $203336.34+412050.7$ & $20333638+4120503$ & 0.79 & $14.85 \pm 0.03$ & $13.48 \pm 0.03$ & $12.82 \pm 0.03$ & AAA & 4.73 \\
\hline 876 & $203336.29+411034.3$ & $20333631+4110345$ & 0.22 & $13.12 \pm 0.02$ & $12.52 \pm 0.01$ & $12.20 \pm 0.02$ & AAA & 1.34 \\
\hline 877 & $203336.40+411818.4$ & $20333640+4118189$ & 0.37 & $15.82 \pm 0.07$ & $14.72 \pm 0.07$ & $14.31 \pm 0.09$ & AAA & N/A \\
\hline 878 & $203336.67+411543.1$ & $20333669+4115429$ & 0.41 & $15.58 \pm 0.06$ & $14.21 \pm 0.05$ & $13.58 \pm 0.05$ & AAA & 7.51 \\
\hline 879 & $203336.64+410846.5$ & - & - & - & - & - & - & - \\
\hline 880 & $203336.69+411509.4$ & $20333682+4115096$ & 1.86 & 16.13 & $14.95 \pm 0.09$ & $14.04 \pm 0.07$ & UAA & N/A \\
\hline 881 & $203336.98+411610.9$ & $20333700+4116113$ & 0.14 & $8.68 \pm 0.01$ & $8.17 \pm 0.01$ & $7.93 \pm 0.01$ & AAA & 6.14 \\
\hline 882 & $203337.00+411157.8$ & $20333705+4111580$ & 0.77 & $16.30 \pm 0.10$ & $15.28 \pm 0.09$ & $14.77 \pm 0.12$ & AAB & N/A \\
\hline 883 & $203337.41+412028.9$ & - & - & - & - & - & - & - \\
\hline 884 & $203337.47+412101.6$ & - & - & - & - & - & - & - \\
\hline 885 & $203337.58+412048.6$ & $20333755+4120490$ & 0.48 & 16.47 & $15.31 \pm 0.11$ & $14.78 \pm 0.13$ & UAB & N/A \\
\hline 886 & $203337.80+411640.3$ & $20333783+4116403$ & 0.50 & $13.94 \pm 0.02$ & $12.82 \pm 0.02$ & $12.34 \pm 0.02$ & AAA & 7.15 \\
\hline 887 & $203338.01+412036.0$ & $20333815+4120355$ & 2.20 & $16.01 \pm 0.08$ & $14.92 \pm 0.06$ & $14.48 \pm 0.10$ & AAA & N/A \\
\hline 888 & $203338.24+411833.4$ & $20333823+4118334$ & 0.29 & $15.96 \pm 0.08$ & $14.87 \pm 0.06$ & $14.30 \pm 0.09$ & AAA & N/A \\
\hline 889 & $203338.37+411620.4$ & $20333840+4116200$ & 0.69 & $12.91 \pm 0.02$ & $12.55 \pm 0.02$ & $12.47 \pm 0.02$ & AAA & N/A \\
\hline 890 & $203338.56+411914.3$ & $20333860+4$ & 0.80 & $14.29 \pm 0.10$ & $13.69 \pm 0.13$ & 11.65 & EBU & 7.26 \\
\hline 891 & $203338.62+412012.2$ & $20333862+4120119$ & 0.56 & $15.94 \pm 0.08$ & $14.70 \pm 0.06$ & $14.35 \pm 0.09$ & AAA & 2.60 \\
\hline 892 & $203338.67+411234.0$ & $20333868+4112334$ & 0.81 & $15.88 \pm 0.07$ & $14.77 \pm 0.06$ & $14.32 \pm 0.09$ & AAA & N/A \\
\hline 893 & $203338.98+411933.4$ & $20333906+4119331$ & 1.11 & 11.22 & $12.52 \pm 0.06$ & 10.70 & UAU & 11.86 \\
\hline 894 & $203339.05+411435.8$ & $20333903+4114362$ & 0.46 & 15.35 & $14.10 \pm 0.05$ & $13.61 \pm 0.05$ & UAA & 23.71 \\
\hline 895 & $203339.07+411925.7$ & $20333910+4119258$ & 0.47 & $7.23 \pm 0.01$ & $6.74 \pm 0.01$ & $6.48 \pm 0.01$ & AAA & 4.26 \\
\hline 896 & $203339.02+410833.8$ & $20333902+4108340$ & 0.03 & $14.24 \pm 0.03$ & $12.90 \pm 0.02$ & $12.19 \pm 0.02$ & AAA & 6.74 \\
\hline 897 & $203339.15+411359.3$ & $20333920+4113586$ & 1.12 & $15.47 \pm 0.05$ & $14.45 \pm 0.05$ & $13.83 \pm 0.05$ & AAA & 8.40 \\
\hline 898 & $203339.22+410648.2$ & $20333928+4106470$ & 1.59 & $15.01 \pm 0.05$ & $13.56 \pm 0.05$ & $12.96 \pm 0.04$ & AAA & 10.66 \\
\hline 899 & $203339.30+411558.5$ & - & - & - & - & - & - & - \\
\hline 900 & $203339.52+411513.3$ & $20333955+4115133$ & 0.40 & 16.05 & 15.34 & $14.81 \pm 0.14$ & UUB & 7.92 \\
\hline 901 & $203339.47+410634.2$ & $20333954+4106334$ & 1.37 & $16.80 \pm 0.17$ & $15.63 \pm 0.13$ & 15.15 & $\mathrm{CBU}$ & 3.50 \\
\hline 902 & $203339.64+411923.3$ & - & - & - & - & - & - & - \\
\hline 903 & $203339.64+411706.5$ & $20333967+4117066$ & 0.34 & $12.42 \pm 0.02$ & $11.69=$ & $11.30 \pm$ & AAA & N/A \\
\hline 904 & $203339.71+411524.9$ & - & - & - & - & - & - & - \\
\hline 905 & $203339.79+411641.1$ & $20333986+4116411$ & 1.01 & $13.36 \pm 0.04$ & $12.49 \pm 0.04$ & $12.04 \pm 0.03$ & AAA & 6.15 \\
\hline 906 & $203339.90+411532.8$ & $20333987+4115328$ & 0.72 & $15.44 \pm 0.06$ & 13.71 & 13.19 & AUU & 9.65 \\
\hline 907 & $203340.42+411445.7$ & - & - & - & - & - & - & - \\
\hline 908 & $203340.59+411630.3$ & - & - & - & - & - & - & - \\
\hline 909 & $203340.66+411442.8$ & $20334060+4114438$ & 1.28 & 12.27 & 11.44 & $10.93 \pm 0.03$ & UUA & N/A \\
\hline 910 & $203340.74+411931.0$ & $20334077+4119308$ & 0.53 & $14.97 \pm 0.08$ & $13.75 \pm 0.04$ & $13.25 \pm 0.05$ & AAA & 9.69 \\
\hline
\end{tabular}


Table 2. continued.

\begin{tabular}{|c|c|c|c|c|c|c|c|c|}
\hline \multirow{2}{*}{$\begin{array}{l}N_{\mathrm{x}} \\
\#\end{array}$} & \multicolumn{2}{|c|}{ X-ray - 2MASS counterpart } & \multirow{2}{*}{$\begin{array}{l}\text { Off. } \\
\left({ }^{\prime \prime}\right)\end{array}$} & \multicolumn{4}{|c|}{ 2MASS photometry } & \multirow{2}{*}{$\begin{array}{l}\begin{array}{l}A_{\mathrm{v}} \\
(\mathrm{mag})\end{array} \\
\text { (a) }\end{array}$} \\
\hline & CXOAC J+ & 2MASS J+ & & $J$ & $\bar{H}$ & $K_{\mathrm{s}}$ & Ph. qual & \\
\hline 911 & $203340.74+411415.2$ & - & - & - & - & - & - & - \\
\hline 912 & $203340.91+410935.5$ & $20334092+4109362$ & 0.50 & $16.13 \pm 0.09$ & $14.90 \pm 0.09$ & $14.24 \pm 0.09$ & AAA & 2.09 \\
\hline 913 & $203341.03+412115.5$ & - & - & - & - & - & - & - \\
\hline 914 & $203340.97+410849.8$ & - & - & - & - & - & - & - \\
\hline 915 & $203341.19+411432.5$ & $20334106+4114334$ & 2.09 & $16.18 \pm 0.11$ & $14.74 \pm 0.08$ & $13.90 \pm 0.08$ & BAA & 15.77 \\
\hline 916 & $203341.27+411455.0$ & $20334124+4114540$ & 1.28 & $16.20 \pm 0.14$ & $14.60 \pm 0.08$ & 13.13 & BAU & 14.53 \\
\hline 917 & $203341.27+411454.9$ & $20334124+4114540$ & 1.28 & $16.20 \pm 0.14$ & $14.60 \pm 0.08$ & 13.13 & BAU & N/A \\
\hline 918 & $203341.38+411513.1$ & $20334143+4115130$ & 0.83 & $16.19 \pm 0.09$ & $14.26 \pm 0.03$ & $12.87 \pm 0.03$ & AAA & 6.13 \\
\hline 919 & $203341.35+410732.0$ & $20334128+4107342$ & 2.30 & $15.14 \pm 0.04$ & $14.60 \pm 0.06$ & $14.45 \pm 0.12$ & $\mathrm{AAB}$ & 7.21 \\
\hline 920 & $203341.52+411858.8$ & $20334158+4118590$ & 0.77 & $12.98 \pm 0.02$ & $12.32 \pm 0.02$ & $11.94 \pm 0.02$ & AAA & 6.32 \\
\hline 921 & $203341.50+411321.9$ & $20334151+4113225$ & 0.39 & $15.10 \pm 0.07$ & $13.62 \pm 0.18$ & $12.65 \pm 0.03$ & ACE & 5.44 \\
\hline 922 & $203341.60+411234.5$ & - & - & - & - & - & - & - \\
\hline 923 & $203341.84+411019.9$ & - & - & - & - & - & - & - \\
\hline 924 & $203341.94+412116.1$ & - & - & - & - & - & - & - \\
\hline 925 & $203341.93+411128.7$ & - & - & - & - & - & - & - \\
\hline 926 & $203341.99+411055.1$ & $20334204+4110549$ & 0.80 & $16.36 \pm 0.12$ & $15.24 \pm 0.10$ & $14.53 \pm 0.11$ & BAB & N/A \\
\hline 927 & $203342.18+410753.8$ & - & - & - & - & - & - & - \\
\hline 928 & $203342.25+411503.2$ & $20334228+4$ & 0.33 & 13 & 0.04 & 12.33 & AAA & 6.75 \\
\hline 929 & $203342.28+411145.8$ & $20334234+4111456$ & 0.75 & $9.89 \pm 0.02$ & $9.38 \pm 0.02$ & $9.10 \pm 0.01$ & AAA & N/A \\
\hline 930 & $203342.52+411456.4$ & $20334254+4114566$ & 0.11 & $10.74 \pm 0.02$ & $10.23 \pm 0.02$ & $9.89 \pm 0.01$ & AAA & 5.98 \\
\hline 931 & $203342.68+411734.5$ & $20334268+4117350$ & 0.34 & $12.52 \pm 0.02$ & $11.99 \pm 0.02$ & $11.59 \pm 0.01$ & AAA & 7.27 \\
\hline 932 & $203342.97+411237.0$ & $20334307+4112376$ & 1.37 & $14.66 \pm 0.04$ & $13.88 \pm 0.05$ & 13.27 & AAU & 3.08 \\
\hline 933 & $203343.00+411504.3$ & - & - & - & - & - & - & - \\
\hline 934 & $203343.26+411033.6$ & $20334329+4110343$ & 0.62 & $14.95 \pm 0.04$ & $13.64 \pm 0.02$ & $13.19 \pm 0.04$ & AAA & 4.84 \\
\hline 935 & $203343.47+411718.3$ & $20334347+4117185$ & 0.12 & $15.76 \pm 0.07$ & $14.54 \pm 0.05$ & $13.98 \pm 0.07$ & AAA & N/A \\
\hline 936 & $203343.47+411101.1$ & $20334333+4111005$ & 2.32 & $13.86 \pm 0.03$ & $13.02 \pm 0.04$ & $12.53 \pm 0.03$ & AAA & 6.73 \\
\hline 937 & $203343.66+411002.3$ & $20334369+4110032$ & 0.72 & $15.72 \pm 0.07$ & $14.43 \pm 0.04$ & $13.97 \pm 0.06$ & AAA & N/A \\
\hline 938 & $203343.80+410917.7$ & $20334383+4109185$ & 0.73 & $14.13 \pm 0.02$ & $12.97 \pm 0.02$ & $12.39 \pm 0.02$ & AAA & 3.80 \\
\hline 939 & $203343.98+411611.9$ & - & - & - & - & - & - & - \\
\hline 940 & $203344.14+411939.2$ & $20334414+4119396$ & 0.19 & $15.77 \pm 0.07$ & $14.50 \pm 0.05$ & $13.72 \pm 0.05$ & AAA & 4.61 \\
\hline 941 & $203344.34+410522.0$ & $20334435+4105199$ & 2.35 & $11.97 \pm 0.01$ & $11.34 \pm 0.01$ & $10.95 \pm 0.01$ & AAA & N/A \\
\hline 942 & $203344.46+411052.5$ & $20334448+4110529$ & 0.18 & $13.27 \pm 0.02$ & $12.33 \pm 0.03$ & $11.91 \pm 0.02$ & AAA & 0.27 \\
\hline 943 & $203344.58+411444.6$ & - & - & - & - & - & - & - \\
\hline 944 & $203344.53+410821.3$ & $20334448+4108218$ & 0.93 & $15.60 \pm 0.06$ & $14.48 \pm 0.07$ & $14.07 \pm 0.08$ & AAA & N/A \\
\hline 945 & $203344.61+410906.2$ & $20334461+4109067$ & 0.29 & $14.51 \pm 0.03$ & $13.28 \pm 0.02$ & $12.92 \pm$ & AAA & 16.69 \\
\hline 946 & $203344.69+411419.8$ & - & - & - & - & - & - & - \\
\hline 947 & $203344.67+411002.2$ & $20334465+4110025$ & 0.45 & 15.95 & $14.95 \pm 0.07$ & 14.25 & UAU & 6.73 \\
\hline 948 & $203344.88+411344.6$ & $20334492+4113445$ & 0.65 & 15.75 & 14.49 & $14.23 \pm 0.09$ & UUA & N/A \\
\hline 949 & $203344.91+411331.4$ & $20334492+4113321$ & 0.44 & $14.63 \pm 0.03$ & $13.45 \pm 0.03$ & $12.94 \pm 0.03$ & AAA & 5.96 \\
\hline 950 & $203345.00+412114.7$ & $20334503+4121151$ & 0.46 & $13.55 \pm 0.02$ & $12.70 \pm 0.02$ & $12.28 \pm 0.02$ & AAA & N/A \\
\hline 951 & $203345.06+412132.6$ & $20334504+4121326$ & 0.48 & $14.47 \pm 0.03$ & $13.19 \pm 0.03$ & $12.40 \pm 0.03$ & AAA & 8.84 \\
\hline 952 & $203345.29+411503.8$ & - & - & - & - & - & - & - \\
\hline 953 & $203345.38+411731.2$ & $20334542+4117310$ & 0.71 & $14.91 \pm 0.04$ & $13.77 \pm 0.05$ & $13.16 \pm 0.04$ & AAA & 12.71 \\
\hline 954 & $203345.41+411621.7$ & $20334543+4116214$ & 0.57 & $16.29 \pm 0.10$ & $15.07 \pm 0.07$ & $14.47 \pm 0.10$ & AAA & 1.78 \\
\hline 955 & $203345.40+411430.7$ & $20334544+4114301$ & 0.96 & 16.31 & $14.81 \pm 0.09$ & $13.16 \pm 0.05$ & UAA & N/A \\
\hline 956 & $203345.47+412018.2$ & $20334546+4120178$ & 0.65 & $14.78 \pm 0.04$ & $13.70 \pm 0.04$ & $13.12 \pm 0.04$ & AAA & 9.19 \\
\hline 957 & $203345.38+410936.4$ & $20334544+4109382$ & 1.74 & $12.60 \pm 0.02$ & $11.86 \pm 0.02$ & $11.57 \pm 0.02$ & AAA & 5.31 \\
\hline 958 & $203345.64+411400.7$ & $20334563+4114006$ & 0.42 & $15.33 \pm 0.05$ & $14.17 \pm 0.04$ & $13.57 \pm 0.05$ & AAA & 4.61 \\
\hline 959 & $203345.84+411726.1$ & - & - & - & - & - & - & - \\
\hline 960 & $203345.95+410850.5$ & $20334599+4108500$ & 0.83 & $13.87 \pm($ & $12.96 \pm 0.03$ & $12.57 \pm 0.03$ & AAA & 12.38 \\
\hline 961 & $203346.31+411629.9$ & $20334635+4116303$ & 0.49 & $16.00 \pm 0.09$ & $14.93 \pm 0.06$ & $14.49 \pm 0.10$ & AAA & N/A \\
\hline 962 & $203346.47+411609.9$ & $20334650+4116102$ & 0.23 & $15.90 \pm 0.08$ & $14.73 \pm 0.06$ & $14.04 \pm 0.07$ & AAA & 8.57 \\
\hline 963 & $203346.50+411812.9$ & $20334649+4118136$ & 0.48 & $15.15 \pm 0.04$ & $13.93 \pm 0.04$ & $13.54 \pm 0.05$ & AAA & 3.23 \\
\hline 964 & $203346.83+411354.7$ & - & - & - & - & - & - & - \\
\hline 965 & $203346.79+410801.0$ & $20334680+4108019$ & 0.66 & $10.15 \pm($ & $9.68 \pm 0.01$ & $9.40 \pm 0$. & AAA & N/A \\
\hline 966 & $203346.84+411048.2$ & $20334672+4110495$ & 2.16 & $16.00 \pm 0.08$ & $14.65 \pm 0.06$ & $14.36 \pm 0.09$ & AAA & N/A \\
\hline 967 & $203346.97+411146.3$ & $20334704+4111455$ & 1.50 & $14.91 \pm 0.04$ & $13.63 \pm 0.03$ & $13.17 \pm 0.04$ & AAA & 11.29 \\
\hline 968 & $203347.04+412005.6$ & $20334710+4120061$ & 0.81 & $16.31 \pm 0.11$ & $15.28 \pm 0.12$ & $14.65 \pm 0.13$ & BBB & 2.10 \\
\hline 969 & $203347.04+410952.6$ & $20334709+4109524$ & 0.81 & 15.11 & $13.82 \pm 0.03$ & $13.38 \pm 0.04$ & UAA & N/A \\
\hline 970 & $203347.20+411316.7$ & $20334713+4113165$ & 1.26 & $14.26 \pm 0.04$ & $13.73 \pm 0.04$ & $13.57 \pm 0.06$ & AAA & 5.98 \\
\hline 971 & $203347.77+412040.9$ & $20334783+4120415$ & 0.83 & $7.99 \pm 0.01$ & $7.49 \pm 0.02$ & $7.21 \pm 0.01$ & AAA & N/A \\
\hline 972 & $203347.92+411537.5$ & $20334794+4115382$ & 0.45 & $15.40 \pm 0.05$ & $14.20 \pm 0.04$ & $13.79 \pm 0.05$ & AAA & N/A \\
\hline 973 & $203347.98+411910.1$ & $20334804+4119105$ & 0.67 & $15.50 \pm 0.04$ & $14.27 \pm 0.04$ & $13.75 \pm 0.06$ & AAA & 9.68 \\
\hline 974 & $203348.10+411842.3$ & - & - & - & - & - & - & - \\
\hline 975 & $203348.20+411346.2$ & - & - & - & - & - & - & - \\
\hline
\end{tabular}


J. F. Albacete Colombo et al.: Unveiling the Cygnus OB2 stellar population with Chandra, Online Material p 39

Table 2. continued.

\begin{tabular}{|c|c|c|c|c|c|c|c|c|}
\hline \multirow{2}{*}{$\begin{array}{l}N_{\mathrm{x}} \\
\#\end{array}$} & \multicolumn{2}{|c|}{ X-ray - 2MASS counterpart } & \multirow{2}{*}{$\begin{array}{l}\text { Off. } \\
\left({ }^{\prime \prime}\right)\end{array}$} & \multicolumn{4}{|c|}{ 2MASS photometry } & \multirow{2}{*}{$\begin{array}{l}A_{\mathrm{v}} \\
\text { (mag) }\end{array}$} \\
\hline & CXOAC J+ & 2MASS J+ & & $J$ & $\bar{H}$ & $\bar{K}_{\mathrm{s}}$ & Ph.qual & \\
\hline 976 & $203348.24+411032.8$ & $20334822+4110330$ & 0.33 & $10.05 \pm 0.01$ & $9.87 \pm 0.01$ & $9.79 \pm 0.01$ & AAA & 4.01 \\
\hline 977 & $203348.63+411824.4$ & $20334868+4118243$ & 0.83 & $14.86 \pm 0.07$ & $13.76 \pm 0.07$ & $13.36 \pm 0.05$ & AAA & 10.28 \\
\hline 978 & $203348.84+412108.0$ & $20334894+4121071$ & 1.82 & $15.54 \pm 0.05$ & $14.25 \pm 0.04$ & $13.72 \pm 0.06$ & AAA & N/A \\
\hline 979 & $203348.87+411940.2$ & $20334884+4119406$ & 0.57 & $10.03 \pm 0.02$ & $9.62 \pm 0.02$ & $9.37 \pm 0.01$ & AAA & 7.28 \\
\hline 980 & $203348.91+411410.4$ & $20334899+4114089$ & 2.02 & $12.63 \pm 0.02$ & $74 \pm 0.02$ & $11.16 \pm 0.01$ & AAA & 16.15 \\
\hline 981 & $9.31+411634.3$ & $20334930+4116358$ & 1.36 & $16.19 \pm 0.09$ & $1.66 \pm 0.06$ & $13.48 \pm$ & AAA & 8.88 \\
\hline 982 & $9.32+411021.8$ & $20334944+4110220$ & 1.53 & $5 \pm 0.06$ & $13 \pm 0.06$ & $13.68 \pm$ & AAA & N/A \\
\hline 983 & $203349.51+411148.6$ & 203349 & 1.07 & $2 \pm 0.07$ & $.68 \pm 0.06$ & $14.21 \pm$ & AAA & 7.44 \\
\hline 984 & $203350.36+411416.3$ & $20335035+4114170$ & 0.50 & $15.05 \pm 0.04$ & $07 \pm 0.03$ & $13.64 \pm 0.05$ & AAA & N/A \\
\hline 985 & $203350.47+411803.1$ & $20335050+4118027$ & 0.73 & $15.86 \pm 0.07$ & $14.83 \pm 0.07$ & $14.29 \pm 0.09$ & AAA & N/A \\
\hline 986 & $203350.82+411616.1$ & $20335078+4116149$ & 1.49 & $13.13 \pm 0.03$ & $12.35 \pm 0.04$ & $11.93 \pm 0.02$ & AAA & 4.23 \\
\hline 987 & $203350.79+410857.4$ & $20335081+4108567$ & 0.88 & $16.19 \pm 0.11$ & $15.08 \pm 0.10$ & $14.56 \pm 0.13$ & BAB & 3.47 \\
\hline 988 & $203351.21+412029.2$ & $20335127+4120288$ & 1.06 & $15.54 \pm 0.05$ & $14.42 \pm 0.04$ & $13.97 \pm 0.06$ & AAA & 8.70 \\
\hline 989 & $203351.48+411358.4$ & $20335151+4113584$ & 0.27 & $15.45 \pm 0.07$ & $14.41 \pm 0.07$ & $14.09 \pm 0.09$ & AAA & N/A \\
\hline 990 & $1.65+411539.0$ & $20335172+4115397$ & 1.09 & $16.75 \pm 0.16$ & $5.65 \pm 0.12$ & $14.89 \pm$ & CBB & 5.47 \\
\hline 991 & 8.8 & 203 & 0.44 & \pm 0.09 & $7 \pm 0.06$ & $14.25 \pm 0.08$ & AAA & 7.44 \\
\hline 992 & $3.10+411118.8$ & $20335310+4$ & 0.30 & $2 \pm 0.02$ & $13.24 \pm 0.02$ & $13.07 \pm 0.03$ & AAA & 5.81 \\
\hline 993 & $.58+411754.9$ & 2033 & 0.89 & 03 & \pm 0.04 & $0 \pm 0.04$ & AAA & 10.46 \\
\hline 994 & $89+412004.0$ & 203 & 1.32 & 15 & $14.30 \pm 0.04$ & $13.80 \pm$ & AAA & 7.20 \\
\hline 995 & 203 & 203 & 1.10 & 15 & $14.26 \pm 0.05$ & 13. & UAA & 2.13 \\
\hline 996 & $5.18+411419.7$ & 2033 & 0.27 & $13.17 \pm$ & $12.73 \pm 0.02$ & $12.50 \pm$ & AAA & N/A \\
\hline 997 & $203355.54+411851.4$ & 203355 & 0.37 & $14.09 \pm$ & $13.03 \pm 0.07$ & $12.58 \pm$ & AAA & N/A \\
\hline 998 & $203356.32+412038.9$ & $20335633+4120391$ & 0.11 & $15.18 \pm 0$ & $14.08 \pm 0.06$ & $13.44 \pm$ & AAA & N/A \\
\hline 999 & $203357.91+411803.1$ & - & - & - & - & - & - & - \\
\hline 1000 & $203358.41+412055.7$ & 2033 & 1.23 & 13 & $12.67 \pm 0.03$ & 12.30 & AAU & N/A \\
\hline 1001 & $203359.65+411737.2$ & $20335952+4117354$ & 2.80 & $8.53 \pm 0.01$ & $8.14 \pm 0.03$ & $7.89 \pm$ & AAA & 0.58 \\
\hline 1002 & $203401.23+412010.4$ & $20340128+4120106$ & 0.66 & $13.18 \pm 0.02$ & $12.59 \pm 0.02$ & $12.31 \pm 0.02$ & AAA & N/A \\
\hline 1003 & $203401.44+411905.4$ & $20340141+4119058$ & 0.56 & $14.78 \pm 0.04$ & $13.66 \pm 0.04$ & $13.17 \pm 0.05$ & AAA & 7.69 \\
\hline
\end{tabular}


J. F. Albacete Colombo et al.: Unveiling the Cygnus OB2 stellar population with Chandra, Online Material p 40

Table 3. X-ray source spectroscopy.

\begin{tabular}{|c|c|c|c|c|c|c|}
\hline $\begin{array}{l}N_{\mathrm{x}} \\
\#\end{array}$ & $\begin{array}{l}\text { NetCnts } \\
\text { (ph.) }\end{array}$ & $\begin{array}{l}\log \left(N_{\mathrm{H}}\right) \\
\left(\mathrm{cm}^{-2}\right)\end{array}$ & $\begin{array}{l}k T \\
(\mathrm{keV})\end{array}$ & $\begin{array}{l}\log (\mathrm{EM}) \\
\left(\mathrm{cm}^{-3}\right)\end{array}$ & $\begin{array}{l}\text { Stat. } \\
\left(\chi_{v}^{2}\right)\end{array}$ & 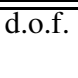 \\
\hline 1 & 67 & $22.13 \pm 0.26$ & 9.99 & 53.70 & 0.34 & 17 \\
\hline 2 & 72 & $21.05 \pm 0.34$ & $0.74 \pm 0.12$ & 53.40 & 1.54 & 17 \\
\hline 3 & 35 & $21.75 \pm 0.03$ & 9.99 & 53.40 & 0.38 & 9 \\
\hline 5 & 55 & $22.15 \pm 0.27$ & $6.05 \pm 8.52$ & 53.40 & 0.81 & 14 \\
\hline 6 & 216 & $22.13 \pm 0.68$ & $5.52 \pm 3.06$ & 54.10 & 0.96 & 19 \\
\hline 7 & 49 & $21.86 \pm 0.04$ & 9.99 & 53.40 & 0.78 & 15 \\
\hline 8 & 33 & $21.70 \pm 0.08$ & 9.99 & 53.40 & 0.51 & 9 \\
\hline 9 & 64 & $21.44 \pm 0.03$ & 9.99 & 53.40 & 0.76 & 14 \\
\hline 10 & 50 & $21.60 \pm 0.10$ & $1.04 \pm 0.23$ & 53.40 & 1.00 & 12 \\
\hline 12 & 99 & $22.14 \pm 0.47$ & $2.19 \pm 0.91$ & 53.88 & 1.02 & 24 \\
\hline 13 & 44 & $22.42 \pm 0.29$ & $3.74 \pm 3.87$ & 53.70 & 1.20 & 10 \\
\hline 14 & 75 & $22.51 \pm 0.30$ & 9.99 & 53.70 & 1.10 & 19 \\
\hline 15 & 60 & $21.90 \pm 0.24$ & $6.85 \pm 8.19$ & 53.40 & 0.66 & 14 \\
\hline 16 & 30 & $22.53 \pm 0.10$ & $2.72 \pm 2.67$ & 53.70 & 0.82 & 7 \\
\hline 17 & 3173 & $22.16 \pm 0.25$ & $1.34 \pm 0.64$ & 55.65 & 1.57 & 252 \\
\hline 19 & 25 & $22.48 \pm 0.15$ & $3.43 \pm 4.19$ & 53.40 & 0.56 & 6 \\
\hline 20 & 49 & $22.26 \pm 0.30$ & $3.04 \pm 1.86$ & 53.70 & 0.72 & 11 \\
\hline 22 & 34 & $21.83 \pm 0.03$ & $3.15 \pm 3.35$ & 53.40 & 1.24 & 7 \\
\hline 23 & 50 & $21.73 \pm 0.11$ & $6.04 \pm 6.96$ & 53.40 & 0.96 & 12 \\
\hline 24 & 26 & $21.98 \pm 0.05$ & 9.99 & 53.40 & 0.56 & 6 \\
\hline 25 & 27 & $22.59 \pm 0.39$ & $0.69 \pm 0.28$ & 54.51 & 0.85 & 5 \\
\hline 26 & 39 & $21.63 \pm 0.03$ & $5.76 \pm 8.14$ & 52.40 & 0.93 & 8 \\
\hline 27 & 70 & $22.27 \pm 0.51$ & $1.85 \pm 0.63$ & 53.88 & 0.90 & 15 \\
\hline 28 & 43 & $22.27 \pm 0.27$ & $2.16 \pm 1.33$ & 53.40 & 1.58 & 10 \\
\hline 29 & 33 & $22.20 \pm 0.75$ & 9.99 & 52.40 & 1.06 & 6 \\
\hline 30 & 41 & $21.96 \pm 0.28$ & $1.04 \pm 0.43$ & 53.40 & 1.04 & 10 \\
\hline 31 & 26 & $22.13 \pm 0.35$ & $0.54 \pm 0.45$ & 54.00 & 0.35 & 5 \\
\hline 32 & 26 & $22.74 \pm 1.22$ & 9.99 & 52.40 & 0.42 & 4 \\
\hline 33 & 232 & $21.82 \pm 0.60$ & $4.59 \pm 1.58$ & 54.10 & 0.79 & 20 \\
\hline 34 & 74 & $22.16 \pm 0.41$ & $2.01 \pm 0.78$ & 53.70 & 1.23 & 16 \\
\hline 35 & 46 & $21.70 \pm 0.16$ & $2.79 \pm 1.84$ & 53.40 & 0.87 & 9 \\
\hline 36 & 45 & $21.19 \pm 0.18$ & $1.37 \pm 0.36$ & 53.40 & 0.52 & 8 \\
\hline 37 & 55 & $22.27 \pm 0.32$ & $4.76 \pm 4.84$ & 53.40 & 0.74 & 12 \\
\hline 38 & 29 & $22.42 \pm 0.25$ & $1.27 \pm 0.67$ & 53.88 & 0.49 & 6 \\
\hline 39 & 91 & $21.79 \pm 0.32$ & 9.99 & 53.70 & 0.62 & 19 \\
\hline 40 & 109 & $21.84 \pm 0.35$ & $2.10 \pm 0.68$ & 53.70 & 0.86 & 25 \\
\hline 41 & 31 & $22.34 \pm 0.28$ & $1.22 \pm 0.61$ & 53.70 & 0.93 & 7 \\
\hline 42 & 23 & $21.59 \pm 0.30$ & 9.99 & 52.40 & 0.75 & 7 \\
\hline 43 & 53 & $22.24 \pm 0.26$ & 8.61 & 53.40 & 0.74 & 11 \\
\hline 44 & 83 & $22.02 \pm 0.28$ & $1.63 \pm 0.66$ & 53.88 & 0.66 & 22 \\
\hline 46 & 20 & $22.46 \pm 0.19$ & $1.24 \pm 0.84$ & 53.70 & 1.19 & 3 \\
\hline 47 & 42 & $22.17 \pm 0.28$ & $1.88 \pm 0.90$ & 53.70 & 0.46 & 10 \\
\hline 48 & 45 & $21.74 \pm 0.16$ & $5.09 \pm 4.48$ & 53.40 & 0.57 & 10 \\
\hline 49 & 53 & $22.47 \pm 0.45$ & $2.05 \pm 0.99$ & 53.88 & 1.25 & 11 \\
\hline 50 & 78 & $22.00 \pm 0.33$ & $1.23 \pm 0.38$ & 53.88 & 0.90 & 23 \\
\hline 51 & 32 & $22.06 \pm 0.01$ & 8.62 & 52.40 & 1.48 & 7 \\
\hline 52 & 156 & $22.39 \pm 0.71$ & $3.02 \pm 0.99$ & 54.25 & 1.00 & 19 \\
\hline 53 & 43 & $22.11 \pm 0.39$ & $1.14 \pm 0.38$ & 53.70 & 0.48 & 10 \\
\hline 54 & 73 & $21.85 \pm 0.19$ & 9.99 & 53.40 & 0.54 & 18 \\
\hline 55 & 33 & $22.11 \pm 0.29$ & $1.39 \pm 0.63$ & 53.40 & 1.32 & 5 \\
\hline 56 & 62 & $22.44 \pm 0.53$ & $1.48 \pm 0.42$ & 54.18 & 0.39 & 14 \\
\hline 57 & 30 & $22.61 \pm 0.39$ & $1.40 \pm 0.51$ & 54.10 & 0.25 & 5 \\
\hline 58 & 49 & $22.08 \pm 0.27$ & $2.73 \pm 1.60$ & 53.40 & 0.53 & 11 \\
\hline 60 & 14913 & $22.30 \pm 0.82$ & $1.24 \pm 0.19$ & 56.49 & 1.40 & 141 \\
\hline 61 & 40 & $22.12 \pm 0.24$ & $4.80 \pm 5.04$ & 53.40 & 0.53 & 8 \\
\hline 62 & 23 & $21.97 \pm 0.04$ & $4.00 \pm 5.57$ & 52.40 & 1.05 & 5 \\
\hline 63 & 24 & $21.78 \pm 0.14$ & 9.99 & 52.40 & 1.07 & 5 \\
\hline 64 & 77 & $22.03 \pm 0.36$ & 9.99 & 53.40 & 0.72 & 17 \\
\hline 67 & 40 & $22.02 \pm 0.15$ & $3.92 \pm 3.64$ & 53.40 & 1.06 & 10 \\
\hline 68 & 27 & $22.52 \pm 0.39$ & $0.90 \pm 0.33$ & 54.10 & 0.91 & 4 \\
\hline 69 & 22 & $22.22 \pm 0.16$ & $3.29 \pm 3.19$ & 53.40 & 0.30 & 3 \\
\hline 71 & 68 & $22.07 \pm 0.32$ & $1.59 \pm 0.60$ & 53.88 & 0.60 & 15 \\
\hline 72 & 107 & $21.75 \pm 0.38$ & $3.16 \pm 1.29$ & 53.70 & 1.50 & 12 \\
\hline 73 & 138 & $22.08 \pm 0.45$ & $2.72 \pm 1.16$ & 54.00 & 1.33 & 18 \\
\hline 74 & 89 & $22.26 \pm 0.59$ & $1.43 \pm 0.35$ & 54.00 & 1.01 & 19 \\
\hline
\end{tabular}


J. F. Albacete Colombo et al.: Unveiling the Cygnus OB2 stellar population with Chandra, Online Material p 41

Table 3. continued.

\begin{tabular}{|c|c|c|c|c|c|c|}
\hline $\begin{array}{l}N_{\mathrm{x}} \\
\#\end{array}$ & $\begin{array}{l}\text { NetCnts } \\
\text { (ph.) }\end{array}$ & $\begin{array}{l}\log \left(N_{\mathrm{H}}\right) \\
\left(\mathrm{cm}^{-2}\right)\end{array}$ & $\begin{array}{l}k T \\
(\mathrm{keV})\end{array}$ & $\begin{array}{l}\log (\mathrm{EM}) \\
\left(\mathrm{cm}^{-3}\right)\end{array}$ & $\begin{array}{l}\text { Stat. } \\
\left(\chi_{v}^{2}\right)\end{array}$ & $\overline{\text { d.o.f. }}$ \\
\hline 76 & 38 & $21.98 \pm 0.11$ & $3.15 \pm 3.19$ & 53.40 & 0.81 & 10 \\
\hline 77 & 38 & $22.12 \pm 0.22$ & $2.11 \pm 1.27$ & 53.40 & 1.04 & 8 \\
\hline 81 & 47 & $22.14 \pm 0.32$ & $2.72 \pm 1.68$ & 53.40 & 0.67 & 10 \\
\hline 82 & 27 & $21.20 \pm 0.29$ & 9.99 & 52.40 & 0.20 & 4 \\
\hline 84 & 22 & $22.65 \pm 0.20$ & $0.54 \pm 0.50$ & 54.85 & 0.97 & 3 \\
\hline 85 & 29 & $22.30 \pm 0.35$ & $1.15 \pm 0.44$ & 53.70 & 0.40 & 5 \\
\hline 87 & 52 & $22.16 \pm 0.25$ & $5.97 \pm 9.26$ & 53.40 & 1.00 & 13 \\
\hline 88 & 37 & $22.29 \pm 0.27$ & $4.49 \pm 5.18$ & 53.40 & 0.60 & 7 \\
\hline 92 & 21 & $22.86 \pm 1.38$ & 9.99 & 52.40 & 0.64 & 3 \\
\hline 93 & 45 & $22.11 \pm 0.33$ & $2.53 \pm 1.40$ & 54.00 & 0.41 & 8 \\
\hline 94 & 80 & $21.78 \pm 0.16$ & $2.66 \pm 1.08$ & 53.40 & 0.68 & 21 \\
\hline 97 & 22 & $21.74 \pm 0.07$ & 6.33 & 52.40 & 0.97 & 3 \\
\hline 100 & 28 & $22.35 \pm 0.29$ & $1.36 \pm 0.67$ & 53.70 & 0.20 & 4 \\
\hline 102 & 37 & $22.18 \pm 0.26$ & $2.72 \pm 1.66$ & 53.40 & 0.34 & 7 \\
\hline 104 & 77 & $21.64 \pm 0.26$ & $5.17 \pm 3.06$ & 53.40 & 0.57 & 17 \\
\hline 105 & 24 & $22.60 \pm 0.43$ & $0.43 \pm 0.15$ & 55.08 & 0.50 & 3 \\
\hline 106 & 53 & $22.59 \pm 0.41$ & $1.56 \pm 0.66$ & 54.10 & 1.41 & 11 \\
\hline 107 & 33 & $21.99 \pm 0.06$ & $4.04 \pm 5.07$ & 53.40 & 0.72 & 9 \\
\hline 114 & 43 & $21.42 \pm 0.14$ & 9.99 & 53.40 & 0.82 & 9 \\
\hline 121 & 24 & $21.95 \pm 0.09$ & $5.77 \pm 9.05$ & 52.40 & 0.30 & 3 \\
\hline 122 & 66 & $22.30 \pm 0.46$ & $3.86 \pm 2.46$ & 53.70 & 0.99 & 13 \\
\hline 123 & 41 & $21.78 \pm 0.16$ & 9.56 & 53.40 & 0.51 & 7 \\
\hline 124 & 63 & $22.04 \pm 0.32$ & $1.20 \pm 0.43$ & 53.70 & 1.22 & 15 \\
\hline 125 & 121 & $22.13 \pm 0.54$ & $4.02 \pm 2.05$ & 53.88 & 1.10 & 15 \\
\hline 128 & 63 & $22.36 \pm 0.46$ & $1.84 \pm 0.78$ & 53.88 & 0.87 & 15 \\
\hline 131 & 31 & $21.76 \pm 0.01$ & $2.61 \pm 2.03$ & 53.40 & 0.73 & 6 \\
\hline 132 & 87 & $22.21 \pm 0.52$ & $2.64 \pm 1.06$ & 53.70 & 1.35 & 18 \\
\hline 133 & 72 & $22.09 \pm 0.79$ & $0.62 \pm 0.17$ & 54.35 & 1.10 & 15 \\
\hline 137 & 24 & $22.00 \pm 0.07$ & $2.03 \pm 1.51$ & 53.40 & 0.64 & 3 \\
\hline 140 & 42 & $22.07 \pm 0.29$ & $6.10 \pm 6.99$ & 53.40 & 0.67 & 8 \\
\hline 141 & 55 & $21.97 \pm 0.32$ & $3.08 \pm 1.76$ & 53.40 & 1.07 & 11 \\
\hline 142 & 186 & $22.15 \pm 0.72$ & $2.44 \pm 0.61$ & 54.18 & 1.26 & 22 \\
\hline 143 & 28 & $22.25 \pm 0.32$ & $1.89 \pm 0.94$ & 53.40 & 0.38 & 4 \\
\hline 146 & 27 & $22.08 \pm 0.08$ & 9.99 & 53.40 & 0.34 & 4 \\
\hline 147 & 22 & $22.03 \pm 0.13$ & 6.30 & 52.40 & 2.00 & 3 \\
\hline 148 & 29 & $22.12 \pm 0.23$ & $1.76 \pm 0.99$ & 53.40 & 0.59 & 5 \\
\hline 149 & 33 & $21.68 \pm 0.02$ & 8.04 & 53.40 & 0.27 & 7 \\
\hline 152 & 53 & $22.23 \pm 0.34$ & $2.08 \pm 1.11$ & 53.70 & 0.92 & 10 \\
\hline 156 & 22 & $22.36 \pm 0.21$ & $1.88 \pm 1.27$ & 53.40 & 0.41 & 2 \\
\hline 158 & 21 & $22.55 \pm 0.31$ & $1.17 \pm 0.57$ & 54.00 & 0.21 & 2 \\
\hline 159 & 21 & $22.15 \pm 0.07$ & $1.67 \pm 1.33$ & 53.40 & 0.42 & 3 \\
\hline 160 & 22 & $22.26 \pm 0.22$ & $2.19 \pm 1.54$ & 53.40 & 0.29 & 3 \\
\hline 161 & 33 & $22.51 \pm 0.21$ & 9.99 & 53.40 & 0.92 & 5 \\
\hline 164 & 85 & $22.08 \pm 0.51$ & $1.74 \pm 0.53$ & 53.88 & 0.82 & 19 \\
\hline 165 & 106 & $22.20 \pm 0.56$ & $1.91 \pm 0.57$ & 54.00 & 1.53 & 11 \\
\hline 166 & 35 & $22.52 \pm 0.49$ & $0.93 \pm 0.33$ & 54.25 & 1.03 & 6 \\
\hline 169 & 41 & $22.53 \pm 0.15$ & 9.99 & 53.40 & 0.89 & 11 \\
\hline 170 & 39 & $22.17 \pm 0.30$ & $2.47 \pm 1.48$ & 53.40 & 1.22 & 6 \\
\hline 172 & 219 & $22.22 \pm 0.75$ & $3.81 \pm 1.27$ & 54.30 & 1.21 & 18 \\
\hline 177 & 51 & $22.51 \pm 0.33$ & 9.99 & 53.70 & 1.12 & 10 \\
\hline 178 & 103 & $22.26 \pm 0.64$ & $2.31 \pm 0.70$ & 54.00 & 0.77 & 12 \\
\hline 179 & 44 & $22.29 \pm 0.34$ & $2.16 \pm 1.09$ & 53.70 & 1.00 & 8 \\
\hline 180 & 25 & $21.48 \pm 0.10$ & 7.19 & 52.40 & 0.25 & 4 \\
\hline 182 & 42 & $22.10 \pm 0.20$ & $2.17 \pm 1.43$ & 53.70 & 0.71 & 10 \\
\hline 183 & 28 & $22.36 \pm 0.33$ & $1.21 \pm 0.53$ & 54.25 & 0.29 & 4 \\
\hline 184 & 65 & $21.95 \pm 0.22$ & 9.99 & 53.40 & 0.74 & 17 \\
\hline 187 & 49 & $22.10 \pm 0.31$ & $3.69 \pm 2.62$ & 53.40 & 0.87 & 9 \\
\hline 188 & 27 & $22.65 \pm 0.47$ & $0.78 \pm 0.31$ & 54.51 & 0.32 & 4 \\
\hline 189 & 24 & $21.74 \pm 0.05$ & 8.31 & 52.40 & 0.73 & 3 \\
\hline 190 & 64 & $22.16 \pm 0.43$ & $3.56 \pm 2.05$ & 53.40 & 0.83 & 13 \\
\hline 193 & 58 & $22.27 \pm 0.51$ & $2.41 \pm 1.02$ & 53.70 & 0.60 & 11 \\
\hline 194 & 23 & $22.50 \pm 0.30$ & $1.16 \pm 0.62$ & 53.88 & 1.40 & 3 \\
\hline 195 & 59 & $22.29 \pm 0.40$ & $6.84 \pm 8.06$ & 53.40 & 0.87 & 12 \\
\hline 199 & 21 & $22.18 \pm 0.14$ & $2.31 \pm 2.02$ & 53.40 & 1.08 & 2 \\
\hline 200 & 27 & $21.98 \pm 0.09$ & $3.43 \pm 3.04$ & 53.40 & 0.62 & 4 \\
\hline
\end{tabular}


J. F. Albacete Colombo et al.: Unveiling the Cygnus OB2 stellar population with Chandra, Online Material p 42

Table 3. continued.

\begin{tabular}{|c|c|c|c|c|c|c|}
\hline $\begin{array}{l}N_{\mathrm{x}} \\
\#\end{array}$ & $\begin{array}{l}\text { NetCnts } \\
\text { (ph.) }\end{array}$ & $\begin{array}{l}\log \left(N_{\mathrm{H}}\right) \\
\left(\mathrm{cm}^{-2}\right)\end{array}$ & $\begin{array}{l}k T \\
(\mathrm{keV})\end{array}$ & $\begin{array}{l}\log (\mathrm{EM}) \\
\left(\mathrm{cm}^{-3}\right)\end{array}$ & $\begin{array}{l}\text { Stat. } \\
\left(\chi_{v}^{2}\right)\end{array}$ & d.o.f. \\
\hline 202 & 24 & $22.14 \pm 0.15$ & $2.31 \pm 2.19$ & 53.40 & 0.51 & 5 \\
\hline 206 & 71 & $22.31 \pm 0.54$ & $2.21 \pm 0.77$ & 53.88 & 0.90 & 14 \\
\hline 209 & 23 & $22.22 \pm 0.08$ & $1.52 \pm 1.12$ & 53.40 & 1.65 & 3 \\
\hline 218 & 52 & $22.30 \pm 0.46$ & $2.38 \pm 1.03$ & 53.70 & 1.57 & 10 \\
\hline 219 & 83 & $22.32 \pm 0.60$ & $2.59 \pm 0.88$ & 53.88 & 0.83 & 16 \\
\hline 220 & 42 & $22.23 \pm 0.39$ & $2.31 \pm 1.13$ & 53.40 & 1.18 & 7 \\
\hline 224 & 26 & $22.24 \pm 0.25$ & $1.41 \pm 0.69$ & 53.40 & 0.84 & 3 \\
\hline 225 & 25 & $22.11 \pm 0.10$ & 9.99 & 53.40 & 1.82 & 4 \\
\hline 226 & 70 & $22.21 \pm 0.10$ & $2.85 \pm 3.07$ & 53.70 & 0.76 & 14 \\
\hline 227 & 39 & $22.13 \pm 0.19$ & 8.28 & 53.40 & 0.50 & 9 \\
\hline 229 & 21 & $21.99 \pm 0.04$ & 7.56 & 52.40 & 0.20 & 2 \\
\hline 231 & 24 & $22.44 \pm 0.33$ & $1.05 \pm 0.64$ & 53.88 & 1.18 & 4 \\
\hline 233 & 67 & $22.51 \pm 0.41$ & 9.99 & 53.70 & 0.62 & 13 \\
\hline 238 & 67 & $22.05 \pm 0.39$ & $6.85 \pm 7.25$ & 53.40 & 0.55 & 13 \\
\hline 239 & 74 & $22.32 \pm 0.53$ & $2.64 \pm 1.12$ & 53.88 & 0.74 & 15 \\
\hline 242 & 93 & $22.29 \pm 0.61$ & $2.28 \pm 0.77$ & 53.88 & 1.31 & 19 \\
\hline 243 & 54 & $22.09 \pm 0.34$ & $6.84 \pm 7.70$ & 53.40 & 0.88 & 10 \\
\hline 244 & 68 & $22.23 \pm 0.69$ & $0.11 \pm 0.63$ & 57.70 & 1.12 & 12 \\
\hline 245 & 21 & $22.43 \pm 0.33$ & $0.59 \pm 0.30$ & 54.30 & 0.24 & 2 \\
\hline 246 & 32 & $21.69 \pm 0.02$ & 9.99 & 53.40 & 0.36 & 5 \\
\hline 247 & 22 & $21.99 \pm 0.10$ & $4.46 \pm 6.06$ & 52.40 & 2.00 & 3 \\
\hline 248 & 42 & $21.60 \pm 0.07$ & 9.99 & 53.40 & 1.31 & 9 \\
\hline 251 & 22 & $22.17 \pm 0.02$ & 9.99 & 52.40 & 1.17 & 3 \\
\hline 253 & 37 & $22.59 \pm 0.50$ & $0.43 \pm 0.13$ & 55.12 & 1.00 & 6 \\
\hline 254 & 83 & $22.38 \pm 0.65$ & $1.43 \pm 0.30$ & 54.18 & 0.92 & 17 \\
\hline 256 & 53 & $22.46 \pm 0.59$ & $1.09 \pm 0.27$ & 54.25 & 0.63 & 10 \\
\hline 259 & 21 & $22.09 \pm 0.09$ & $2.00 \pm 1.58$ & 53.40 & 0.63 & 2 \\
\hline 260 & 289 & $22.39 \pm 0.86$ & $4.76 \pm 1.60$ & 54.40 & 0.66 & 25 \\
\hline 270 & 71 & $22.11 \pm 0.41$ & $2.61 \pm 1.25$ & 53.70 & 0.64 & 16 \\
\hline 271 & 186 & $22.22 \pm 0.70$ & $4.66 \pm 2.06$ & 54.00 & 1.23 & 22 \\
\hline 272 & 39 & $22.25 \pm 0.31$ & $1.97 \pm 1.12$ & 53.40 & 0.90 & 8 \\
\hline 273 & 23 & $22.33 \pm 0.27$ & $1.94 \pm 1.24$ & 53.40 & 0.50 & 3 \\
\hline 277 & 21 & $22.43 \pm 0.18$ & $1.56 \pm 1.01$ & 53.70 & 0.47 & 2 \\
\hline 279 & 24 & $22.11 \pm 0.09$ & $2.71 \pm 2.90$ & 53.40 & 0.99 & 4 \\
\hline 280 & 106 & $22.29 \pm 0.58$ & $2.66 \pm 1.04$ & 54.00 & 1.33 & 12 \\
\hline 283 & 346 & $22.30 \pm 0.86$ & $3.85 \pm 1.02$ & 54.40 & 1.11 & 30 \\
\hline 284 & 25 & $22.09 \pm 0.12$ & $4.02 \pm 4.57$ & 53.40 & 0.65 & 4 \\
\hline 290 & 21 & $22.55 \pm 0.19$ & $1.25 \pm 0.84$ & 53.88 & 0.20 & 3 \\
\hline 294 & 40 & $22.26 \pm 0.27$ & $1.47 \pm 0.80$ & 53.70 & 1.00 & 10 \\
\hline 297 & 25 & $22.49 \pm 0.45$ & $0.76 \pm 0.31$ & 54.18 & 1.25 & 3 \\
\hline 299 & 65 & $22.24 \pm 0.55$ & $1.92 \pm 0.59$ & 53.70 & 0.78 & 14 \\
\hline 300 & 270 & $21.81 \pm 0.70$ & $2.27 \pm 0.44$ & 54.10 & 1.98 & 21 \\
\hline 301 & 27 & $22.19 \pm 0.04$ & 9.99 & 53.40 & 1.84 & 4 \\
\hline 302 & 22 & $22.36 \pm 0.19$ & $1.24 \pm 0.79$ & 53.70 & 0.20 & 2 \\
\hline 303 & 21 & $22.43 \pm 0.15$ & $5.10 \pm 8.15$ & 53.40 & 0.57 & 2 \\
\hline 305 & 33 & $22.34 \pm 0.26$ & $1.46 \pm 0.93$ & 53.70 & 0.90 & 8 \\
\hline 306 & 22 & $22.53 \pm 0.43$ & $0.88 \pm 0.32$ & 54.10 & 0.97 & 3 \\
\hline 307 & 22 & $22.19 \pm 0.15$ & $1.25 \pm 0.77$ & 53.40 & 1.02 & 3 \\
\hline 308 & 37 & $22.19 \pm 0.33$ & $1.97 \pm 1.02$ & 53.40 & 0.37 & 6 \\
\hline 309 & 36 & $22.31 \pm 0.38$ & $1.37 \pm 0.54$ & 53.70 & 0.70 & 5 \\
\hline 313 & 41 & $21.97 \pm 0.06$ & 9.99 & 53.40 & 1.18 & 9 \\
\hline 315 & 107 & $22.34 \pm 0.86$ & $0.70 \pm 1.00$ & 54.63 & 0.80 & 11 \\
\hline 316 & 27 & $22.38 \pm 0.32$ & $1.36 \pm 0.65$ & 53.70 & 0.51 & 4 \\
\hline 317 & 20 & $21.50 \pm 0.27$ & 9.99 & 52.40 & 1.84 & 2 \\
\hline 319 & 20 & $23.18 \pm 0.32$ & $0.87 \pm 0.30$ & 55.16 & 1.82 & 2 \\
\hline 320 & 27 & $21.99 \pm 0.05$ & 9.99 & 52.40 & 0.79 & 4 \\
\hline 324 & 47 & $22.33 \pm 0.44$ & $1.99 \pm 0.90$ & 53.70 & 0.86 & 8 \\
\hline 326 & 47 & $22.23 \pm 0.33$ & $1.39 \pm 0.63$ & 53.70 & 1.10 & 11 \\
\hline 327 & 97 & $22.19 \pm 0.49$ & $3.19 \pm 1.34$ & 54.00 & 1.09 & 22 \\
\hline 329 & 23 & $22.13 \pm 0.04$ & 8.20 & 52.40 & 0.88 & 5 \\
\hline 330 & 67 & $22.21 \pm 0.46$ & $4.10 \pm 2.53$ & 53.70 & 0.80 & 13 \\
\hline 335 & 141 & $22.20 \pm 0.60$ & $4.18 \pm 2.06$ & 53.88 & 1.37 & 17 \\
\hline 336 & 74 & $22.41 \pm 0.54$ & $3.87 \pm 2.41$ & 53.70 & 1.11 & 15 \\
\hline 341 & 31 & $22.49 \pm 0.37$ & $1.49 \pm 0.66$ & 53.88 & 1.15 & 4 \\
\hline 345 & 22 & $22.08 \pm 0.10$ & 9.32 & 52.40 & 0.23 & 3 \\
\hline
\end{tabular}


J. F. Albacete Colombo et al.: Unveiling the Cygnus OB2 stellar population with Chandra, Online Material p 43

Table 3. continued.

\begin{tabular}{|c|c|c|c|c|c|c|}
\hline $\begin{array}{l}N_{\mathrm{x}} \\
\#\end{array}$ & $\begin{array}{l}\text { NetCnts } \\
\text { (ph.) }\end{array}$ & $\begin{array}{l}\log \left(N_{\mathrm{H}}\right) \\
\left(\mathrm{cm}^{-2}\right)\end{array}$ & $\begin{array}{l}k T \\
(\mathrm{keV})\end{array}$ & $\begin{array}{l}\log (\mathrm{EM}) \\
\left(\mathrm{cm}^{-3}\right)\end{array}$ & $\begin{array}{l}\text { Stat. } \\
\left(\chi_{v}^{2}\right)\end{array}$ & $\overline{\text { d.o.f. }}$ \\
\hline 351 & 36 & $22.58 \pm 0.53$ & $0.82 \pm 0.32$ & 54.72 & 0.66 & 5 \\
\hline 352 & 68 & $22.23 \pm 0.48$ & $3.12 \pm 1.42$ & 53.70 & 0.73 & 13 \\
\hline 354 & 31 & $21.84 \pm 0.10$ & $3.33 \pm 2.74$ & 52.40 & 1.20 & 4 \\
\hline 355 & 30 & $22.21 \pm 0.24$ & $2.17 \pm 1.37$ & 53.40 & 0.78 & 6 \\
\hline 356 & 33 & $22.54 \pm 0.40$ & $1.38 \pm 0.47$ & 53.88 & 0.99 & 5 \\
\hline 358 & 24 & $22.38 \pm 0.23$ & $1.30 \pm 0.77$ & 53.70 & 0.49 & 3 \\
\hline 359 & 62 & $22.43 \pm 0.57$ & $2.76 \pm 1.16$ & 53.88 & 0.67 & 12 \\
\hline 361 & 59 & $22.40 \pm 0.51$ & $1.29 \pm 0.43$ & 54.10 & 0.48 & 11 \\
\hline 366 & 123 & $22.20 \pm 0.68$ & $2.77 \pm 0.89$ & 54.00 & 0.55 & 14 \\
\hline 372 & 66 & $22.32 \pm 0.47$ & $1.71 \pm 0.64$ & 53.88 & 1.08 & 13 \\
\hline 375 & 21 & $21.25 \pm 0.41$ & 9.99 & 52.40 & 0.20 & 3 \\
\hline 378 & 105 & $22.43 \pm 0.70$ & $1.89 \pm 0.46$ & 54.25 & 0.58 & 11 \\
\hline 379 & 69 & $22.44 \pm 0.33$ & 9.99 & 53.70 & 1.63 & 15 \\
\hline 381 & 89 & $22.49 \pm 0.65$ & $1.48 \pm 0.39$ & 54.30 & 1.07 & 17 \\
\hline 383 & 27 & $22.09 \pm 0.15$ & $4.98 \pm 6.55$ & 53.40 & 1.18 & 3 \\
\hline 384 & 36 & $22.59 \pm 0.42$ & $1.99 \pm 0.99$ & 53.88 & 0.69 & 6 \\
\hline 387 & 31 & $22.48 \pm 0.53$ & $0.57 \pm 0.15$ & 54.66 & 0.39 & 5 \\
\hline 388 & 38 & $21.79 \pm 0.06$ & 9.99 & 52.40 & 1.90 & 6 \\
\hline 389 & 59 & $22.37 \pm 0.51$ & $1.85 \pm 0.59$ & 53.88 & 0.85 & 11 \\
\hline 390 & 26 & $22.28 \pm 0.13$ & 8.63 & 53.40 & 1.41 & 3 \\
\hline 392 & 45 & $22.50 \pm 0.47$ & $1.73 \pm 0.64$ & 53.88 & 1.08 & 8 \\
\hline 393 & 65 & $22.20 \pm 0.53$ & $1.41 \pm 0.40$ & 53.88 & 1.29 & 12 \\
\hline 396 & 43 & $22.11 \pm 0.26$ & 9.99 & 53.40 & 1.20 & 7 \\
\hline 398 & 60 & $22.40 \pm 0.46$ & $1.71 \pm 0.63$ & 54.00 & 0.41 & 11 \\
\hline 399 & 21 & $22.20 \pm 0.02$ & 9.99 & 53.40 & 0.54 & 2 \\
\hline 400 & 32 & $21.96 \pm 0.11$ & 9.99 & 52.40 & 1.50 & 5 \\
\hline 402 & 182 & $22.28 \pm 0.69$ & $7.44 \pm 5.20$ & 54.10 & 0.52 & 21 \\
\hline 404 & 216 & $22.37 \pm 0.76$ & $4.89 \pm 2.05$ & 54.25 & 1.03 & 18 \\
\hline 406 & 110 & $22.27 \pm 0.62$ & $1.94 \pm 0.56$ & 54.10 & 1.06 & 13 \\
\hline 407 & 103 & $22.44 \pm 0.65$ & $1.57 \pm 0.39$ & 54.25 & 1.22 & 11 \\
\hline 408 & 46 & $22.63 \pm 0.49$ & $1.18 \pm 0.38$ & 54.40 & 0.95 & 9 \\
\hline 410 & 65 & $22.17 \pm 0.30$ & 9.99 & 53.40 & 1.50 & 13 \\
\hline 413 & 25 & $22.49 \pm 0.22$ & $1.09 \pm 0.60$ & 53.88 & 1.39 & 3 \\
\hline 414 & 139 & $22.35 \pm 0.73$ & $2.38 \pm 0.61$ & 54.18 & 1.04 & 16 \\
\hline 415 & 25 & $22.29 \pm 0.22$ & $4.99 \pm 6.40$ & 53.40 & 0.62 & 3 \\
\hline 419 & 36 & $22.28 \pm 0.38$ & $2.25 \pm 1.26$ & 53.70 & 0.54 & 7 \\
\hline 420 & 37 & $22.28 \pm 0.35$ & $3.75 \pm 2.96$ & 53.40 & 1.02 & 6 \\
\hline 422 & 76 & $22.41 \pm 0.61$ & $1.00 \pm 0.30$ & 54.51 & 0.99 & 18 \\
\hline 423 & 26 & $22.57 \pm 0.37$ & $1.83 \pm 0.93$ & 53.70 & 0.41 & 3 \\
\hline 424 & 70 & $22.16 \pm 0.51$ & $2.89 \pm 1.24$ & 53.70 & 0.78 & 14 \\
\hline 429 & 21 & $22.52 \pm 0.24$ & $1.54 \pm 0.95$ & 53.70 & 1.00 & 2 \\
\hline 430 & 20 & $22.31 \pm 0.15$ & $3.10 \pm 3.56$ & 53.40 & 1.44 & 2 \\
\hline 432 & 113 & $22.44 \pm 0.67$ & $2.48 \pm 0.78$ & 54.10 & 1.23 & 12 \\
\hline 433 & 1240 & $22.34 \pm 1.28$ & $1.05 \pm 0.76$ & 55.08 & 1.40 & 15 \\
\hline 434 & 47 & $22.41 \pm 0.07$ & $2.32 \pm 1.04$ & 53.70 & 0.97 & 8 \\
\hline 435 & 23 & $22.30 \pm 0.24$ & $2.05 \pm 1.35$ & 53.40 & 0.20 & 3 \\
\hline 436 & 366 & $22.31 \pm 1.13$ & $0.60 \pm 0.53$ & 55.29 & 2.00 & 27 \\
\hline 438 & 28 & $22.48 \pm 0.28$ & $1.62 \pm 1.05$ & 53.70 & 0.74 & 4 \\
\hline 439 & 148 & $22.35 \pm 0.66$ & $2.59 \pm 0.84$ & 54.18 & 1.36 & 17 \\
\hline 440 & 21 & $22.24 \pm 0.15$ & $5.19 \pm 7.13$ & 53.40 & 0.20 & 2 \\
\hline 443 & 26 & $22.35 \pm 0.25$ & $1.60 \pm 0.94$ & 53.70 & 0.65 & 3 \\
\hline 445 & 83 & $22.36 \pm 0.61$ & $3.00 \pm 1.21$ & 53.88 & 0.83 & 17 \\
\hline 446 & 54 & $22.32 \pm 0.46$ & $2.04 \pm 0.83$ & 53.88 & 0.61 & 10 \\
\hline 447 & 51 & $22.23 \pm 0.45$ & $3.55 \pm 2.20$ & 53.40 & 1.09 & 9 \\
\hline 448 & 24 & $22.52 \pm 0.41$ & $0.97 \pm 0.43$ & 54.00 & 0.53 & 3 \\
\hline 449 & 42 & $22.19 \pm 0.38$ & $2.40 \pm 1.16$ & 53.40 & 0.96 & 6 \\
\hline 454 & 37 & $22.42 \pm 0.39$ & $1.74 \pm 0.81$ & 53.70 & 0.66 & 7 \\
\hline 455 & 47 & $22.25 \pm 0.45$ & $2.56 \pm 1.13$ & 53.70 & 0.51 & 8 \\
\hline 456 & 106 & $22.32 \pm 0.81$ & $0.85 \pm 0.21$ & 54.44 & 1.40 & 11 \\
\hline 458 & 34 & $22.15 \pm 0.22$ & $1.45 \pm 0.80$ & 53.70 & 0.85 & 6 \\
\hline 459 & 40 & $22.11 \pm 0.17$ & 8.62 & 53.40 & 1.08 & 8 \\
\hline 460 & 21 & $21.81 \pm 0.13$ & 9.99 & 52.40 & 1.75 & 2 \\
\hline 461 & 54 & $22.41 \pm 0.57$ & $1.73 \pm 0.56$ & 54.10 & 0.82 & 10 \\
\hline 464 & 30 & $22.51 \pm 0.31$ & $1.66 \pm 0.96$ & 53.88 & 0.36 & 5 \\
\hline 466 & 24 & $22.53 \pm 0.16$ & $1.72 \pm 1.34$ & 53.70 & 1.45 & 3 \\
\hline
\end{tabular}


J. F. Albacete Colombo et al.: Unveiling the Cygnus OB2 stellar population with Chandra, Online Material p 44

Table 3. continued.

\begin{tabular}{|c|c|c|c|c|c|c|}
\hline $\begin{array}{l}N_{\mathrm{x}} \\
\#\end{array}$ & $\begin{array}{l}\text { NetCnts } \\
\text { (ph.) }\end{array}$ & $\begin{array}{l}\log \left(N_{\mathrm{H}}\right) \\
\left(\mathrm{cm}^{-2}\right)\end{array}$ & $\begin{array}{l}k T \\
(\mathrm{keV})\end{array}$ & $\begin{array}{l}\log (\text { EM }) \\
\left(\mathrm{cm}^{-3}\right)\end{array}$ & $\begin{array}{l}\text { Stat. } \\
\left(\chi_{v}^{2}\right)\end{array}$ & $\overline{\text { d.o.f. }}$ \\
\hline 467 & 41 & $22.28 \pm 0.22$ & 9.99 & 53.40 & 0.40 & 8 \\
\hline 471 & 147 & $22.19 \pm 0.63$ & $3.91 \pm 1.56$ & 53.88 & 1.74 & 17 \\
\hline 472 & 24 & $23.00 \pm 0.04$ & 6.83 & 53.70 & 0.21 & 3 \\
\hline 475 & 61 & $22.33 \pm 0.48$ & $1.53 \pm 0.50$ & 53.88 & 0.84 & 12 \\
\hline 476 & 41 & $22.63 \pm 0.63$ & $0.89 \pm 0.19$ & 54.51 & 0.82 & 7 \\
\hline 478 & 32 & $22.29 \pm 0.30$ & $3.31 \pm 2.57$ & 53.40 & 0.87 & 5 \\
\hline 479 & 57 & $22.75 \pm 0.51$ & $1.30 \pm 0.49$ & 54.51 & 1.25 & 11 \\
\hline 480 & 158 & $22.36 \pm 0.48$ & $1.62 \pm 0.79$ & 53.88 & 0.96 & 682 \\
\hline 481 & 176 & $22.33 \pm 0.70$ & $3.37 \pm 1.09$ & 54.18 & 1.09 & 21 \\
\hline 482 & 21 & $22.46 \pm 0.33$ & $1.05 \pm 0.66$ & 54.00 & 0.20 & 2 \\
\hline 483 & 48 & $22.43 \pm 0.40$ & $2.50 \pm 1.49$ & 53.70 & 0.90 & 10 \\
\hline 484 & 46 & $22.34 \pm 0.43$ & $1.37 \pm 0.51$ & 53.88 & 1.08 & 8 \\
\hline 485 & 25 & $22.36 \pm 0.19$ & 6.57 & 53.40 & 0.96 & 3 \\
\hline 488 & 9736 & $22.30 \pm 0.66$ & $2.39 \pm 0.77$ & 55.96 & 1.30 & 121 \\
\hline 490 & 37 & $22.65 \pm 0.51$ & $0.92 \pm 0.25$ & 54.80 & 0.56 & 6 \\
\hline 492 & 30 & $22.16 \pm 0.29$ & $1.82 \pm 1.00$ & 53.70 & 1.32 & 4 \\
\hline 495 & 81 & $22.19 \pm 0.80$ & $0.44 \pm 0.74$ & 54.66 & 1.12 & 13 \\
\hline 497 & 143 & $22.31 \pm 0.61$ & $6.84 \pm 5.75$ & 53.88 & 1.44 & 17 \\
\hline 499 & 29 & $22.62 \pm 0.46$ & $1.01 \pm 0.47$ & 54.25 & 0.77 & 4 \\
\hline 500 & 34 & $22.54 \pm 0.49$ & $1.78 \pm 0.67$ & 54.35 & 0.27 & 5 \\
\hline 501 & 32 & $22.42 \pm 0.35$ & $2.81 \pm 2.13$ & 53.70 & 1.71 & 5 \\
\hline 502 & 51 & $22.49 \pm 0.49$ & $1.61 \pm 0.59$ & 54.35 & 0.34 & 9 \\
\hline 503 & 58 & $22.24 \pm 0.42$ & $2.00 \pm 0.96$ & 53.70 & 0.79 & 13 \\
\hline 506 & 22 & $22.46 \pm 0.32$ & $0.91 \pm 0.46$ & 53.88 & 1.40 & 2 \\
\hline 507 & 105 & $22.31 \pm 0.60$ & $3.78 \pm 1.79$ & 53.88 & 1.12 & 11 \\
\hline 508 & 23 & $21.98 \pm 0.15$ & $2.78 \pm 2.60$ & 53.40 & 0.76 & 3 \\
\hline 509 & 26 & $22.63 \pm 0.24$ & $0.90 \pm 0.45$ & 54.25 & 1.70 & 3 \\
\hline 510 & 85 & $22.44 \pm 0.58$ & $1.47 \pm 0.42$ & 54.18 & 1.02 & 18 \\
\hline 511 & 40 & $21.94 \pm 0.09$ & 8.62 & 52.40 & 2.00 & 7 \\
\hline 513 & 26 & $22.58 \pm 0.09$ & 9.99 & 53.40 & 0.31 & 4 \\
\hline 515 & 30 & $22.68 \pm 0.48$ & $0.91 \pm 0.29$ & 54.40 & 1.23 & 4 \\
\hline 519 & 20 & $22.10 \pm 0.05$ & $1.49 \pm 1.16$ & 53.40 & 0.54 & 3 \\
\hline 520 & 78 & $22.44 \pm 0.57$ & $1.29 \pm 0.37$ & 54.18 & 1.07 & 16 \\
\hline 523 & 50 & $22.37 \pm 0.48$ & $2.36 \pm 1.07$ & 53.70 & 0.79 & 9 \\
\hline 525 & 33 & $22.37 \pm 0.32$ & $1.64 \pm 0.88$ & 53.88 & 0.73 & 5 \\
\hline 526 & 22 & $22.62 \pm 0.39$ & $0.93 \pm 0.40$ & 54.18 & 1.10 & 2 \\
\hline 527 & 24 & $22.07 \pm 0.12$ & $4.29 \pm 4.89$ & 53.40 & 0.71 & 3 \\
\hline 529 & 25 & $22.20 \pm 0.22$ & $2.17 \pm 1.54$ & 53.40 & 1.06 & 3 \\
\hline 530 & 137 & $22.27 \pm 0.96$ & $0.70 \pm 0.77$ & 54.60 & 1.10 & 14 \\
\hline 534 & 23 & $22.50 \pm 0.31$ & $0.85 \pm 0.60$ & 54.10 & 0.90 & 3 \\
\hline 535 & 29 & $22.57 \pm 0.41$ & $0.93 \pm 0.34$ & 54.25 & 0.33 & 5 \\
\hline 536 & 28 & $22.35 \pm 0.36$ & $1.17 \pm 0.46$ & 53.70 & 0.25 & 4 \\
\hline 537 & 111 & $22.28 \pm 0.87$ & $0.75 \pm 0.12$ & 54.51 & 1.10 & 11 \\
\hline 539 & 50 & $22.61 \pm 0.46$ & $2.54 \pm 1.50$ & 53.88 & 1.47 & 9 \\
\hline 544 & 1834 & $22.48 \pm 1.46$ & $0.59 \pm 0.21$ & 56.30 & 1.30 & 24 \\
\hline 547 & 42 & $22.63 \pm 0.50$ & $1.15 \pm 0.38$ & 54.35 & 0.58 & 9 \\
\hline 549 & 26 & $22.23 \pm 0.24$ & $3.66 \pm 3.63$ & 53.40 & 0.79 & 3 \\
\hline 552 & 58 & $22.43 \pm 0.56$ & $0.60 \pm 0.18$ & 54.60 & 1.36 & 11 \\
\hline 554 & 21 & $22.59 \pm 0.33$ & $0.58 \pm 0.24$ & 54.66 & 0.20 & 3 \\
\hline 555 & 343 & $22.34 \pm 0.87$ & $2.60 \pm 0.56$ & 54.51 & 2.00 & 29 \\
\hline 556 & 60 & $22.10 \pm 0.31$ & $2.63 \pm 1.48$ & 53.70 & 0.47 & 14 \\
\hline 557 & 130 & $22.14 \pm 0.61$ & $2.76 \pm 0.90$ & 54.00 & 1.41 & 15 \\
\hline 559 & 108 & $22.26 \pm 0.57$ & $1.07 \pm 0.33$ & 54.00 & 1.14 & 12 \\
\hline 561 & 27 & $22.25 \pm 0.19$ & $2.52 \pm 2.03$ & 53.40 & 0.99 & 4 \\
\hline 562 & 290 & $22.34 \pm 1.10$ & $0.55 \pm 0.38$ & 55.37 & 1.00 & 22 \\
\hline 564 & 25 & $22.30 \pm 0.24$ & $1.44 \pm 0.79$ & 53.40 & 0.98 & 5 \\
\hline 567 & 22 & $22.39 \pm 0.19$ & $1.68 \pm 1.21$ & 53.88 & 0.39 & 2 \\
\hline 568 & 13138 & $22.31 \pm 0.72$ & $1.92 \pm 0.45$ & 56.29 & 1.40 & 152 \\
\hline 569 & 26 & $22.31 \pm 0.90$ & 8.81 & 53.40 & 0.85 & 3 \\
\hline 570 & 36 & $22.76 \pm 0.47$ & $0.46 \pm 0.12$ & 55.54 & 0.58 & 7 \\
\hline 571 & 34 & $22.29 \pm 0.36$ & $1.98 \pm 0.94$ & 53.70 & 0.42 & 5 \\
\hline 572 & 24 & $22.39 \pm 0.26$ & $2.03 \pm 1.30$ & 53.40 & 0.20 & 3 \\
\hline 575 & 20 & $22.01 \pm 0.01$ & 5.68 & 52.40 & 2.00 & 2 \\
\hline 577 & 20 & $22.17 \pm 0.13$ & $2.75 \pm 2.60$ & 53.40 & 0.79 & 2 \\
\hline 579 & 94 & $22.22 \pm 0.52$ & $2.63 \pm 0.93$ & 53.88 & 0.74 & 20 \\
\hline
\end{tabular}


J. F. Albacete Colombo et al.: Unveiling the Cygnus OB2 stellar population with Chandra, Online Material p 45

Table 3. continued.

\begin{tabular}{|c|c|c|c|c|c|c|}
\hline $\begin{array}{l}N_{\mathrm{x}} \\
\#\end{array}$ & $\begin{array}{l}\text { NetCnts } \\
\text { (ph.) }\end{array}$ & $\begin{array}{l}\log \left(N_{\mathrm{H}}\right) \\
\left(\mathrm{cm}^{-2}\right)\end{array}$ & $\begin{array}{l}k T \\
(\mathrm{keV})\end{array}$ & $\begin{array}{l}\log (\mathrm{EM}) \\
\left(\mathrm{cm}^{-3}\right)\end{array}$ & $\begin{array}{l}\text { Stat. } \\
\left(\chi_{v}^{2}\right)\end{array}$ & d.o.f. \\
\hline 581 & 50 & $22.23 \pm 0.64$ & $0.22 \pm 0.47$ & 55.54 & 0.69 & 10 \\
\hline 582 & 75 & $22.30 \pm 0.47$ & $1.60 \pm 0.60$ & 54.00 & 1.36 & 16 \\
\hline 584 & 53 & $22.43 \pm 0.59$ & $0.39 \pm 0.15$ & 55.13 & 0.80 & 11 \\
\hline 585 & 31 & $21.40 \pm 0.24$ & 9.99 & 52.40 & 0.89 & 7 \\
\hline 586 & 40 & $22.37 \pm 0.37$ & $1.48 \pm 0.72$ & 53.70 & 1.08 & 7 \\
\hline 588 & 26 & $22.54 \pm 0.47$ & $0.70 \pm 0.20$ & 54.40 & 0.73 & 5 \\
\hline 590 & 58 & $22.35 \pm 0.39$ & $2.37 \pm 1.30$ & 53.70 & 1.34 & 12 \\
\hline 593 & 65 & $22.16 \pm 0.48$ & $3.55 \pm 2.16$ & 53.40 & 0.74 & 13 \\
\hline 594 & 74 & $22.29 \pm 0.52$ & $1.88 \pm 0.67$ & 53.88 & 1.26 & 16 \\
\hline 595 & 60 & $22.70 \pm 0.58$ & $0.59 \pm 0.15$ & 55.23 & 0.78 & 13 \\
\hline 596 & 327 & $22.17 \pm 0.37$ & $2.54 \pm 3.91$ & 54.44 & 1.76 & 28 \\
\hline 597 & 66 & $22.36 \pm 0.65$ & $0.51 \pm 0.18$ & 54.92 & 1.00 & 13 \\
\hline 599 & 50 & $22.30 \pm 0.37$ & $4.96 \pm 4.58$ & 53.40 & 1.01 & 10 \\
\hline 600 & 428 & $22.19 \pm 0.96$ & $1.23 \pm 0.13$ & 54.83 & 0.90 & 35 \\
\hline 601 & 48 & $21.32 \pm 0.18$ & 9.99 & 53.40 & 1.33 & 11 \\
\hline 603 & 53 & $22.37 \pm 0.54$ & $1.47 \pm 0.40$ & 54.00 & 0.64 & 9 \\
\hline 606 & 55 & $22.30 \pm 0.29$ & $1.10 \pm 0.70$ & 54.35 & 0.90 & 11 \\
\hline 608 & 36 & $22.48 \pm 0.37$ & $1.34 \pm 0.66$ & 54.00 & 0.33 & 6 \\
\hline 610 & 22 & $22.42 \pm 0.17$ & $1.67 \pm 1.28$ & 53.40 & 0.82 & 2 \\
\hline 612 & 118 & $22.47 \pm 0.78$ & $1.38 \pm 0.24$ & 54.48 & 1.03 & 12 \\
\hline 615 & 135 & $22.43 \pm 0.86$ & $0.55 \pm 0.62$ & 55.11 & 1.00 & 14 \\
\hline 616 & 55 & $22.25 \pm 0.36$ & $5.43 \pm 4.86$ & 53.40 & 0.59 & 12 \\
\hline 618 & 41 & $22.38 \pm 0.09$ & 2.91 & 54.00 & 0.94 & 8 \\
\hline 619 & 24 & $21.99 \pm 0.00$ & $4.11 \pm 6.28$ & 52.40 & 1.21 & 4 \\
\hline 620 & 68 & $22.14 \pm 0.51$ & $3.52 \pm 1.79$ & 53.70 & 0.52 & 13 \\
\hline 622 & 139 & $22.22 \pm 0.69$ & $2.88 \pm 0.87$ & 54.00 & 0.98 & 15 \\
\hline 623 & 33 & $22.17 \pm 0.29$ & $2.50 \pm 1.62$ & 53.70 & 0.63 & 6 \\
\hline 624 & 25 & $22.31 \pm 0.18$ & $1.45 \pm 0.90$ & 53.40 & 1.64 & 4 \\
\hline 625 & 430 & $22.11 \pm 1.03$ & $0.48 \pm 0.52$ & 55.37 & 1.00 & 32 \\
\hline 626 & 120 & $22.05 \pm 0.44$ & $1.37 \pm 0.36$ & 54.00 & 1.00 & 14 \\
\hline 627 & 120 & $22.05 \pm 0.45$ & $1.37 \pm 0.37$ & 54.00 & 1.50 & 14 \\
\hline 629 & 44 & $22.30 \pm 0.46$ & $1.39 \pm 0.46$ & 53.70 & 1.10 & 8 \\
\hline 632 & 25 & $22.30 \pm 0.25$ & $2.04 \pm 1.33$ & 53.40 & 0.43 & 4 \\
\hline 633 & 144 & $22.37 \pm 0.63$ & $1.69 \pm 0.47$ & 54.30 & 1.51 & 16 \\
\hline 634 & 36 & $22.11 \pm 0.14$ & 9.99 & 53.40 & 1.12 & 7 \\
\hline 635 & 33 & $21.76 \pm 0.01$ & 9.99 & 52.40 & 1.22 & 5 \\
\hline 637 & 31 & $22.28 \pm 0.25$ & $3.22 \pm 2.86$ & 53.40 & 0.76 & 5 \\
\hline 643 & 93 & $22.53 \pm 0.66$ & $1.97 \pm 0.60$ & 54.18 & 0.70 & 19 \\
\hline 646 & 27 & $22.35 \pm 0.36$ & $1.45 \pm 0.62$ & 53.88 & 0.38 & 4 \\
\hline 649 & 41 & $21.68 \pm 0.00$ & 9.29 & 52.40 & 1.25 & 9 \\
\hline 650 & 119 & $22.02 \pm 0.77$ & $0.86 \pm 0.15$ & 54.10 & 0.92 & 13 \\
\hline 652 & 212 & $22.44 \pm 0.79$ & $3.25 \pm 0.97$ & 54.35 & 2.00 & 17 \\
\hline 656 & 27 & $22.39 \pm 0.37$ & $1.15 \pm 0.79$ & 53.70 & 1.13 & 4 \\
\hline 659 & 67 & $22.29 \pm 0.46$ & $1.37 \pm 0.45$ & 54.00 & 1.28 & 14 \\
\hline 660 & 21 & $22.15 \pm 0.05$ & 9.99 & 53.40 & 1.92 & 3 \\
\hline 661 & 31 & $22.53 \pm 0.44$ & $0.89 \pm 0.28$ & 54.25 & 0.98 & 5 \\
\hline 664 & 33 & $21.88 \pm 0.01$ & 9.99 & 53.40 & 1.41 & 6 \\
\hline 666 & 34 & $22.23 \pm 0.24$ & $1.86 \pm 1.12$ & 53.70 & 0.55 & 7 \\
\hline 667 & 35 & $22.30 \pm 0.34$ & $2.01 \pm 1.13$ & 53.70 & 0.56 & 7 \\
\hline 668 & 34 & $21.87 \pm 0.13$ & $3.23 \pm 2.37$ & 53.40 & 0.58 & 6 \\
\hline 670 & 82 & $22.49 \pm 0.61$ & $1.52 \pm 0.42$ & 54.30 & 0.77 & 17 \\
\hline 671 & 46 & $22.28 \pm 0.46$ & $1.93 \pm 0.79$ & 53.70 & 0.69 & 8 \\
\hline 672 & 86 & $22.36 \pm 0.64$ & $2.32 \pm 0.72$ & 54.00 & 0.58 & 18 \\
\hline 675 & 64 & $23.15 \pm 0.21$ & 9.99 & 54.00 & 1.20 & 14 \\
\hline 676 & 186 & $22.12 \pm 0.95$ & $0.79 \pm 0.12$ & 54.51 & 0.81 & 21 \\
\hline 683 & 30 & $22.38 \pm 0.36$ & $2.35 \pm 1.45$ & 53.40 & 0.67 & 4 \\
\hline 685 & 35 & $22.06 \pm 0.27$ & $4.61 \pm 4.76$ & 53.40 & 0.54 & 6 \\
\hline 686 & 36 & $22.39 \pm 0.49$ & $0.35 \pm 0.97$ & 55.02 & 1.10 & 5 \\
\hline 687 & 91 & $22.26 \pm 0.52$ & $4.95 \pm 3.37$ & 53.70 & 0.89 & 17 \\
\hline 688 & 39 & $21.94 \pm 0.12$ & $4.29 \pm 4.71$ & 53.40 & 0.74 & 9 \\
\hline 690 & 21 & $22.56 \pm 0.00$ & $1.71 \pm 1.87$ & 53.70 & 1.87 & 3 \\
\hline 693 & 23 & $22.13 \pm 0.08$ & 9.99 & 52.40 & 2.00 & 3 \\
\hline 694 & 46 & $22.29 \pm 0.45$ & $1.95 \pm 0.85$ & 53.88 & 0.39 & 9 \\
\hline 695 & 32 & $22.18 \pm 0.33$ & $2.30 \pm 1.27$ & 53.40 & 0.49 & 5 \\
\hline 700 & 57 & $22.57 \pm 0.61$ & $1.13 \pm 0.26$ & 54.40 & 0.71 & 11 \\
\hline
\end{tabular}


J. F. Albacete Colombo et al.: Unveiling the Cygnus OB2 stellar population with Chandra, Online Material p 46

Table 3. continued.

\begin{tabular}{|c|c|c|c|c|c|c|}
\hline $\begin{array}{l}N_{\mathrm{x}} \\
\#\end{array}$ & $\begin{array}{l}\text { NetCnts } \\
\text { (ph.) }\end{array}$ & $\begin{array}{l}\log \left(N_{\mathrm{H}}\right) \\
\left(\mathrm{cm}^{-2}\right)\end{array}$ & $\begin{array}{l}k T \\
(\mathrm{keV})\end{array}$ & $\begin{array}{l}\log (\mathrm{EM}) \\
\left(\mathrm{cm}^{-3}\right)\end{array}$ & $\begin{array}{l}\text { Stat. } \\
\left(\chi_{v}^{2}\right)\end{array}$ & d.o.f. \\
\hline 701 & 65 & $22.05 \pm 0.34$ & 8.22 & 53.40 & 1.12 & 13 \\
\hline 702 & 42 & $22.32 \pm 0.36$ & $1.72 \pm 0.78$ & 53.70 & 0.55 & 9 \\
\hline 703 & 20 & $21.95 \pm 0.14$ & 6.85 & 52.40 & 1.54 & 2 \\
\hline 705 & 49 & $22.56 \pm 0.59$ & $1.40 \pm 0.34$ & 54.18 & 0.62 & 9 \\
\hline 708 & 97 & $22.19 \pm 0.58$ & $2.27 \pm 0.71$ & 53.88 & 0.63 & 20 \\
\hline 709 & 28 & $22.76 \pm 0.31$ & $1.14 \pm 0.48$ & 54.30 & 1.43 & 5 \\
\hline 710 & 81 & $22.42 \pm 0.61$ & $1.98 \pm 0.65$ & 54.00 & 0.92 & 17 \\
\hline 711 & 28 & $22.18 \pm 0.22$ & $1.37 \pm 0.67$ & 53.40 & 0.45 & 4 \\
\hline 712 & 209 & $22.34 \pm 0.79$ & $2.31 \pm 0.54$ & 54.35 & 2.00 & 16 \\
\hline 718 & 37 & $22.43 \pm 0.38$ & $1.64 \pm 0.76$ & 53.88 & 0.34 & 6 \\
\hline 721 & 389 & $22.01 \pm 0.17$ & $0.61 \pm 0.49$ & 54.78 & 2.00 & 28 \\
\hline 722 & 161 & $21.04 \pm 0.56$ & $1.00 \pm 0.13$ & 54.51 & 0.68 & 26 \\
\hline 724 & 165 & $22.23 \pm 0.74$ & $2.74 \pm 0.77$ & 54.10 & 0.68 & 20 \\
\hline 725 & 32 & $21.95 \pm 0.12$ & 9.99 & 53.40 & 0.46 & 5 \\
\hline 727 & 22 & $22.11 \pm 0.04$ & 9.99 & 53.40 & 0.37 & 2 \\
\hline 728 & 28 & $22.17 \pm 0.46$ & $0.20 \pm 0.12$ & 55.44 & 0.20 & 4 \\
\hline 729 & 3731 & $22.15 \pm 0.40$ & $1.89 \pm 0.80$ & 55.55 & 1.10 & 49 \\
\hline 731 & 25 & $22.51 \pm 0.49$ & $0.73 \pm 0.23$ & 54.51 & 2.00 & 3 \\
\hline 732 & 33 & $22.28 \pm 0.27$ & $2.31 \pm 1.45$ & 53.40 & 1.13 & 5 \\
\hline 733 & 61 & $22.37 \pm 0.40$ & $1.63 \pm 0.72$ & 53.88 & 1.37 & 12 \\
\hline 734 & 57 & $22.35 \pm 0.59$ & $1.37 \pm 0.33$ & 54.00 & 0.77 & 11 \\
\hline 736 & 59 & $22.24 \pm 0.43$ & $2.52 \pm 1.22$ & 53.70 & 0.63 & 13 \\
\hline 738 & 21 & $22.74 \pm 0.22$ & $0.62 \pm 0.47$ & 55.01 & 2.00 & 2 \\
\hline 739 & 37 & $22.46 \pm 0.50$ & $0.93 \pm 0.33$ & 54.18 & 0.71 & 6 \\
\hline 740 & 28 & $22.18 \pm 0.08$ & 7.17 & 53.40 & 1.35 & 5 \\
\hline 743 & 417 & $22.26 \pm 0.89$ & $5.75 \pm 2.26$ & 54.48 & 1.18 & 36 \\
\hline 744 & 35 & $22.27 \pm 0.30$ & $1.57 \pm 0.80$ & 53.70 & 0.73 & 7 \\
\hline 745 & 29 & $22.21 \pm 0.25$ & $2.12 \pm 1.27$ & 53.70 & 0.78 & 4 \\
\hline 746 & 92 & $22.22 \pm 0.55$ & $4.28 \pm 2.27$ & 54.00 & 0.72 & 19 \\
\hline 750 & 102 & $22.20 \pm 0.55$ & $4.78 \pm 3.11$ & 53.70 & 1.43 & 11 \\
\hline 751 & 50 & $21.93 \pm 0.21$ & $5.94 \pm 7.90$ & 53.40 & 1.25 & 10 \\
\hline 753 & 1416 & $22.20 \pm 1.08$ & 9.99 & 54.91 & 2.00 & 20 \\
\hline 754 & 35 & $22.19 \pm 0.24$ & 9.83 & 53.40 & 0.31 & 6 \\
\hline 759 & 24 & $22.47 \pm 0.28$ & $1.46 \pm 0.76$ & 53.70 & 0.71 & 4 \\
\hline 760 & 29 & $22.44 \pm 0.40$ & $1.03 \pm 0.52$ & 54.00 & 0.82 & 4 \\
\hline 761 & 32 & $21.94 \pm 0.16$ & $2.57 \pm 1.75$ & 53.40 & 0.75 & 6 \\
\hline 762 & 36 & $22.40 \pm 0.42$ & $2.95 \pm 1.87$ & 53.40 & 0.61 & 6 \\
\hline 763 & 117 & $22.46 \pm 0.69$ & $2.03 \pm 0.54$ & 54.25 & 1.10 & 13 \\
\hline 764 & 51 & $22.38 \pm 0.51$ & $1.17 \pm 0.32$ & 54.00 & 0.99 & 9 \\
\hline 769 & 30 & $22.25 \pm 0.28$ & $1.86 \pm 0.98$ & 53.40 & 0.59 & 4 \\
\hline 770 & 64 & $22.31 \pm 0.51$ & $2.58 \pm 1.07$ & 53.70 & 0.84 & 12 \\
\hline 771 & 22 & $22.61 \pm 0.01$ & 9.99 & 53.40 & 1.15 & 4 \\
\hline 772 & 24 & $22.32 \pm 0.17$ & $3.36 \pm 3.66$ & 53.40 & 1.95 & 3 \\
\hline 773 & 38 & $22.27 \pm 0.37$ & $1.37 \pm 0.54$ & 53.70 & 0.77 & 6 \\
\hline 774 & 23 & $22.65 \pm 0.87$ & $3.83 \pm 5.41$ & 52.40 & 1.28 & 5 \\
\hline 775 & 29 & $22.22 \pm 0.22$ & $4.66 \pm 6.36$ & 53.40 & 1.07 & 5 \\
\hline 777 & 121 & $22.39 \pm 0.74$ & $1.39 \pm 0.26$ & 54.35 & 1.48 & 13 \\
\hline 779 & 331 & $22.03 \pm 0.79$ & $2.44 \pm 0.48$ & 54.30 & 1.20 & 25 \\
\hline 781 & 28 & $22.43 \pm 0.39$ & $1.16 \pm 0.44$ & 53.88 & 0.35 & 4 \\
\hline 784 & 44 & $22.47 \pm 0.52$ & $1.16 \pm 0.32$ & 54.18 & 0.36 & 8 \\
\hline 785 & 26 & $22.56 \pm 0.37$ & $1.17 \pm 0.51$ & 54.00 & 1.20 & 3 \\
\hline 786 & 153 & $22.31 \pm 0.74$ & $1.89 \pm 0.44$ & 54.25 & 1.20 & 18 \\
\hline 787 & 22 & $22.22 \pm 0.12$ & $4.98 \pm 7.58$ & 53.40 & 0.86 & 2 \\
\hline 791 & 28 & $22.02 \pm 0.09$ & $1.93 \pm 1.57$ & 53.40 & 0.91 & 5 \\
\hline 794 & 46 & $22.07 \pm 0.19$ & $1.96 \pm 1.11$ & 53.40 & 1.64 & 9 \\
\hline 795 & 23 & $21.83 \pm 0.05$ & 9.99 & 52.40 & 0.85 & 3 \\
\hline 796 & 370 & $22.29 \pm 0.88$ & $4.22 \pm 1.09$ & 54.44 & 2.00 & 31 \\
\hline 797 & 38 & $22.15 \pm 0.28$ & $3.29 \pm 2.61$ & 53.40 & 1.30 & 7 \\
\hline 798 & 139 & $22.12 \pm 0.53$ & $5.24 \pm 3.04$ & 53.88 & 1.36 & 17 \\
\hline 801 & 31 & $22.48 \pm 0.42$ & $1.03 \pm 0.48$ & 54.00 & 1.23 & 4 \\
\hline 803 & 27 & $22.20 \pm 0.23$ & $1.95 \pm 1.21$ & 53.40 & 0.34 & 4 \\
\hline 806 & 21 & $21.88 \pm 0.13$ & 9.99 & 52.40 & 1.14 & 3 \\
\hline 808 & 31 & $22.30 \pm 0.34$ & $1.89 \pm 1.02$ & 53.70 & 0.48 & 5 \\
\hline 809 & 52 & $22.30 \pm 0.44$ & $1.66 \pm 0.65$ & 53.88 & 0.82 & 9 \\
\hline 811 & 33 & $22.50 \pm 0.56$ & $0.86 \pm 0.27$ & 54.25 & 0.26 & 5 \\
\hline
\end{tabular}


J. F. Albacete Colombo et al.: Unveiling the Cygnus OB2 stellar population with Chandra, Online Material p 47

Table 3. continued.

\begin{tabular}{|c|c|c|c|c|c|c|}
\hline $\begin{array}{l}N_{\mathrm{x}} \\
\#\end{array}$ & $\begin{array}{l}\text { NetCnts } \\
\text { (ph.) }\end{array}$ & $\begin{array}{l}\log \left(N_{\mathrm{H}}\right) \\
\left(\mathrm{cm}^{-2}\right)\end{array}$ & $\begin{array}{l}k T \\
(\mathrm{keV})\end{array}$ & $\begin{array}{l}\log (\mathrm{EM}) \\
\left(\mathrm{cm}^{-3}\right)\end{array}$ & $\begin{array}{l}\text { Stat. } \\
\left(\chi_{v}^{2}\right)\end{array}$ & d.o.f. \\
\hline 813 & 63 & $22.00 \pm 0.36$ & $3.07 \pm 1.71$ & 53.40 & 1.02 & 13 \\
\hline 818 & 168 & $22.19 \pm 0.68$ & $4.91 \pm 2.10$ & 54.00 & 1.00 & 20 \\
\hline 821 & 30 & $21.73 \pm 0.01$ & 9.99 & 53.40 & 0.52 & 5 \\
\hline 822 & 21 & $22.37 \pm 0.22$ & $1.11 \pm 0.54$ & 53.70 & 1.46 & 2 \\
\hline 824 & 34 & $22.66 \pm 0.44$ & $0.31 \pm 0.15$ & 55.75 & 2.00 & 5 \\
\hline 828 & 228 & $22.35 \pm 0.81$ & $1.57 \pm 0.27$ & 54.58 & 1.90 & 18 \\
\hline 830 & 26 & $22.37 \pm 0.31$ & $2.01 \pm 1.23$ & 53.40 & 0.68 & 4 \\
\hline 831 & 72 & $22.55 \pm 0.63$ & $1.12 \pm 0.23$ & 54.55 & 0.52 & 15 \\
\hline 833 & 36 & $23.22 \pm 0.06$ & 9.99 & 53.88 & 0.79 & 10 \\
\hline 834 & 173 & $22.22 \pm 0.64$ & $7.78 \pm 6.21$ & 54.00 & 1.68 & 21 \\
\hline 837 & 33 & $22.35 \pm 0.33$ & $2.73 \pm 2.01$ & 53.40 & 1.33 & 5 \\
\hline 838 & 23 & $21.99 \pm 0.09$ & $4.01 \pm 4.46$ & 52.40 & 0.63 & 3 \\
\hline 840 & 59 & $21.38 \pm 0.04$ & 9.99 & 53.40 & 0.41 & 16 \\
\hline 842 & 53 & $22.26 \pm 0.42$ & $2.11 \pm 0.93$ & 53.70 & 0.51 & 11 \\
\hline 847 & 34 & $22.31 \pm 0.28$ & $2.01 \pm 1.15$ & 53.70 & 0.41 & 7 \\
\hline 848 & 25 & $22.09 \pm 0.16$ & $1.77 \pm 1.12$ & 53.40 & 0.66 & 3 \\
\hline 849 & 59 & $22.21 \pm 0.39$ & $2.48 \pm 1.26$ & 53.70 & 1.00 & 11 \\
\hline 851 & 24 & $22.83 \pm 0.08$ & 9.99 & 53.70 & 1.43 & 3 \\
\hline 854 & 34 & $22.27 \pm 0.26$ & $2.16 \pm 1.42$ & 53.70 & 0.64 & 7 \\
\hline 856 & 37 & $22.14 \pm 0.33$ & $2.87 \pm 1.84$ & 53.40 & 0.45 & 7 \\
\hline 857 & 113 & $22.33 \pm 0.65$ & $2.03 \pm 0.55$ & 54.18 & 0.99 & 13 \\
\hline 858 & 27 & $22.27 \pm 0.43$ & $0.92 \pm 0.36$ & 53.88 & 0.43 & 4 \\
\hline 860 & 27 & $21.43 \pm 0.24$ & 9.99 & 52.40 & 0.30 & 6 \\
\hline 863 & 268 & $22.25 \pm 0.87$ & $2.81 \pm 0.57$ & 54.40 & 0.83 & 22 \\
\hline 864 & 56 & $22.64 \pm 0.29$ & 9.99 & 53.70 & 0.56 & 12 \\
\hline 865 & 25 & $22.00 \pm 0.03$ & 9.99 & 53.40 & 1.25 & 3 \\
\hline 866 & 34 & $21.77 \pm 0.05$ & 9.39 & 52.40 & 1.10 & 8 \\
\hline 867 & 29 & $21.91 \pm 0.09$ & 9.99 & 53.40 & 0.20 & 4 \\
\hline 868 & 23 & $21.64 \pm 0.08$ & 9.23 & 52.40 & 0.78 & 3 \\
\hline 870 & 42 & $22.93 \pm 1.21$ & 9.99 & 52.40 & 1.19 & 9 \\
\hline 872 & 192 & $22.07 \pm 0.60$ & 9.99 & 54.00 & 0.61 & 24 \\
\hline 874 & 42 & $22.23 \pm 0.22$ & $1.70 \pm 1.03$ & 53.70 & 0.22 & 9 \\
\hline 875 & 20 & $21.81 \pm 0.06$ & $3.95 \pm 5.10$ & 52.40 & 0.41 & 3 \\
\hline 876 & 56 & $21.88 \pm 0.27$ & $4.13 \pm 3.08$ & 53.40 & 0.91 & 12 \\
\hline 877 & 34 & $22.34 \pm 0.30$ & $3.49 \pm 2.87$ & 53.40 & 0.71 & 5 \\
\hline 878 & 45 & $22.16 \pm 0.32$ & $6.84 \pm 8.91$ & 53.40 & 0.65 & 8 \\
\hline 879 & 62 & $22.43 \pm 0.32$ & 9.99 & 53.70 & 0.85 & 14 \\
\hline 881 & 52 & $22.29 \pm 0.65$ & $0.55 \pm 0.13$ & 54.44 & 0.90 & 10 \\
\hline 883 & 33 & $22.56 \pm 0.14$ & 9.99 & 53.70 & 0.90 & 6 \\
\hline 884 & 57 & $22.30 \pm 0.31$ & 9.99 & 53.70 & 0.67 & 12 \\
\hline 885 & 21 & $21.08 \pm 0.68$ & 9.99 & 52.40 & 2.00 & 3 \\
\hline 886 & 49 & $22.46 \pm 0.54$ & $1.12 \pm 0.28$ & 54.18 & 0.47 & 9 \\
\hline 890 & 20 & $21.93 \pm 0.07$ & 6.11 & 52.40 & 0.89 & 2 \\
\hline 891 & 32 & $22.11 \pm 0.19$ & $6.69 \pm 8.97$ & 53.40 & 0.40 & 6 \\
\hline 893 & 75 & $22.11 \pm 0.49$ & $3.43 \pm 1.73$ & 53.70 & 0.47 & 15 \\
\hline 894 & 26 & $22.29 \pm 0.17$ & $1.24 \pm 0.87$ & 53.70 & 1.30 & 4 \\
\hline 895 & 81 & $22.16 \pm 0.77$ & $0.75 \pm 0.16$ & 54.30 & 1.00 & 15 \\
\hline 896 & 102 & $22.11 \pm 0.53$ & $2.99 \pm 1.25$ & 53.88 & 1.04 & 12 \\
\hline 898 & 42 & $22.08 \pm 0.26$ & $2.89 \pm 2.22$ & 53.40 & 0.50 & 9 \\
\hline 901 & 46 & $22.27 \pm 0.11$ & 9.99 & 53.40 & 0.97 & 10 \\
\hline 902 & 31 & $22.25 \pm 0.27$ & $1.60 \pm 0.88$ & 53.70 & 1.00 & 5 \\
\hline 903 & 126 & $22.19 \pm 0.61$ & $2.48 \pm 0.76$ & 54.00 & 1.45 & 14 \\
\hline 905 & 26 & $22.65 \pm 0.12$ & $0.43 \pm 0.56$ & 55.11 & 0.61 & 1 \\
\hline 906 & 28 & $22.10 \pm 0.18$ & $2.72 \pm 2.18$ & 53.40 & 1.27 & 4 \\
\hline 911 & 71 & $22.16 \pm 0.40$ & $5.44 \pm 5.05$ & 53.40 & 0.82 & 14 \\
\hline 912 & 20 & $22.21 \pm 0.28$ & $0.48 \pm 0.36$ & 54.10 & 0.98 & 4 \\
\hline 913 & 82 & $22.15 \pm 0.29$ & $5.23 \pm 4.74$ & 53.70 & 1.32 & 17 \\
\hline 914 & 160 & $21.99 \pm 0.56$ & $2.31 \pm 0.63$ & 54.10 & 1.01 & 19 \\
\hline 916 & 82 & $22.50 \pm 0.61$ & $2.33 \pm 0.80$ & 54.10 & 0.75 & 18 \\
\hline 917 & 82 & $22.50 \pm 0.63$ & $2.23 \pm 0.70$ & 54.10 & 0.75 & 18 \\
\hline 918 & 41 & $22.78 \pm 0.17$ & 9.99 & 53.70 & 0.83 & 7 \\
\hline 919 & 29 & $21.72 \pm 0.20$ & $0.69 \pm 0.24$ & 53.40 & 0.45 & 7 \\
\hline 920 & 68 & $22.35 \pm 0.51$ & $1.26 \pm 0.41$ & 54.10 & 0.99 & 14 \\
\hline 922 & 37 & $22.49 \pm 0.51$ & $1.09 \pm 0.32$ & 54.10 & 0.45 & 7 \\
\hline 923 & 78 & $22.30 \pm 0.56$ & $1.56 \pm 0.44$ & 54.00 & 0.82 & 16 \\
\hline
\end{tabular}


J. F. Albacete Colombo et al.: Unveiling the Cygnus OB2 stellar population with Chandra, Online Material p 48

Table 3. continued.

\begin{tabular}{|c|c|c|c|c|c|c|}
\hline $\begin{array}{l}N_{\mathrm{x}} \\
\#\end{array}$ & $\begin{array}{l}\text { NetCnts } \\
\text { (ph.) }\end{array}$ & $\begin{array}{l}\log \left(N_{\mathrm{H}}\right) \\
\left(\mathrm{cm}^{-2}\right)\end{array}$ & $\begin{array}{l}k T \\
(\mathrm{keV})\end{array}$ & $\begin{array}{l}\log (\mathrm{EM}) \\
\left(\mathrm{cm}^{-3}\right)\end{array}$ & $\begin{array}{l}\text { Stat. } \\
\left(\chi_{v}^{2}\right)\end{array}$ & $\overline{\text { d.o.f. }}$ \\
\hline 924 & 57 & $22.42 \pm 0.31$ & 9.99 & 53.70 & 0.82 & 11 \\
\hline 925 & 44 & $22.11 \pm 0.41$ & $1.14 \pm 0.38$ & 53.70 & 0.79 & 8 \\
\hline 927 & 332 & $22.90 \pm 1.12$ & $0.68 \pm 0.39$ & 54.00 & 0.91 & 24 \\
\hline 928 & 124 & $22.33 \pm 0.66$ & $1.57 \pm 0.34$ & 54.30 & 0.87 & 14 \\
\hline 929 & 23 & $22.02 \pm 0.21$ & $0.99 \pm 1.49$ & 53.40 & 1.54 & 3 \\
\hline 931 & 37 & $21.88 \pm 0.06$ & $6.50 \pm 9.80$ & 53.40 & 1.15 & 7 \\
\hline 932 & 38 & $21.73 \pm 0.31$ & $0.76 \pm 0.19$ & 53.40 & 1.05 & 7 \\
\hline 934 & 53 & $22.27 \pm 0.37$ & $2.64 \pm 1.54$ & 53.70 & 0.72 & 11 \\
\hline 935 & 22 & $22.53 \pm 0.32$ & $0.74 \pm 0.36$ & 54.25 & 1.05 & 3 \\
\hline 937 & 21 & $22.20 \pm 0.05$ & $1.24 \pm 1.12$ & 53.40 & 0.97 & 4 \\
\hline 938 & 34 & $21.79 \pm 0.05$ & $5.10 \pm 6.05$ & 53.40 & 0.52 & 7 \\
\hline 940 & 168 & $22.27 \pm 0.74$ & $2.92 \pm 0.85$ & 54.25 & 0.39 & 21 \\
\hline 941 & 38 & $21.69 \pm 0.06$ & $2.94 \pm 3.65$ & 53.40 & 1.05 & 11 \\
\hline 942 & 49 & $22.25 \pm 0.41$ & $1.73 \pm 0.64$ & 53.70 & 0.74 & 10 \\
\hline 943 & 40 & $22.07 \pm 0.34$ & $2.96 \pm 1.73$ & 53.40 & 0.39 & 7 \\
\hline 944 & 31 & $21.72 \pm 0.00$ & $3.87 \pm 4.67$ & 53.40 & 0.41 & 7 \\
\hline 945 & 125 & $22.18 \pm 0.54$ & $3.43 \pm 1.47$ & 54.00 & 1.00 & 15 \\
\hline 947 & 80 & $22.28 \pm 0.44$ & $5.07 \pm 4.09$ & 53.70 & 1.28 & 18 \\
\hline 948 & 21 & $21.74 \pm 0.19$ & 9.99 & 52.40 & 0.39 & 2 \\
\hline 949 & 54 & $21.86 \pm 0.29$ & $3.18 \pm 1.77$ & 53.40 & 0.38 & 11 \\
\hline 950 & 31 & $22.17 \pm 0.36$ & $1.08 \pm 0.48$ & 53.70 & 0.45 & 7 \\
\hline 951 & 37 & $21.73 \pm 0.05$ & $0.84 \pm 0.64$ & 52.40 & 2.00 & 8 \\
\hline 952 & 29 & $22.11 \pm 0.12$ & 9.70 & 53.40 & 1.07 & 5 \\
\hline 953 & 21 & $22.11 \pm 0.15$ & $2.20 \pm 1.77$ & 53.40 & 1.09 & 3 \\
\hline 956 & 38 & $21.90 \pm 0.16$ & $3.34 \pm 2.58$ & 53.40 & 0.50 & 8 \\
\hline 957 & 71 & $21.87 \pm 0.25$ & 9.99 & 53.40 & 0.57 & 16 \\
\hline 958 & 22 & $21.40 \pm 0.20$ & $5.61 \pm 9.43$ & 52.40 & 0.45 & 3 \\
\hline 959 & 60 & $22.58 \pm 0.34$ & 9.99 & 53.88 & 0.34 & 12 \\
\hline 960 & 33 & $22.75 \pm 1.29$ & 9.99 & 52.40 & 0.80 & 7 \\
\hline 962 & 28 & $21.71 \pm 0.01$ & 9.99 & 52.40 & 0.62 & 5 \\
\hline 964 & 55 & $21.85 \pm 0.24$ & 8.82 & 53.40 & 0.93 & 11 \\
\hline 965 & 65 & $21.74 \pm 0.20$ & $3.43 \pm 2.61$ & 53.40 & 0.80 & 16 \\
\hline 967 & 47 & $22.14 \pm 0.27$ & $2.01 \pm 1.02$ & 53.40 & 0.95 & 10 \\
\hline 968 & 64 & $22.02 \pm 0.35$ & $4.78 \pm 3.77$ & 53.70 & 0.36 & 14 \\
\hline 971 & 50 & $22.23 \pm 0.31$ & $1.28 \pm 0.63$ & 53.88 & 0.90 & 11 \\
\hline 973 & 31 & $22.08 \pm 0.20$ & $2.83 \pm 2.39$ & 53.40 & 0.76 & 6 \\
\hline 974 & 48 & $21.85 \pm 0.11$ & 9.99 & 53.40 & 1.30 & 10 \\
\hline 975 & 44 & $22.63 \pm 0.28$ & 9.99 & 53.70 & 0.79 & 9 \\
\hline 976 & 32 & $22.83 \pm 1.29$ & $0.69 \pm 0.26$ & 52.40 & 0.93 & 6 \\
\hline 977 & 27 & $22.33 \pm 0.38$ & $1.10 \pm 0.43$ & 53.88 & 0.53 & 5 \\
\hline 978 & 25 & $22.22 \pm 0.23$ & $1.42 \pm 0.87$ & 53.70 & 0.33 & 6 \\
\hline 979 & 77 & $22.39 \pm 0.53$ & $2.16 \pm 0.80$ & 54.00 & 1.50 & 17 \\
\hline 981 & 23 & $22.02 \pm 0.04$ & 9.99 & 52.40 & 0.40 & 4 \\
\hline 982 & 27 & $22.52 \pm 0.28$ & $1.24 \pm 0.67$ & 54.00 & 0.71 & 5 \\
\hline 983 & 46 & $21.62 \pm 0.04$ & 9.99 & 53.40 & 0.85 & 10 \\
\hline 985 & 29 & $21.84 \pm 0.04$ & 9.99 & 53.40 & 0.66 & 6 \\
\hline 986 & 28 & $21.83 \pm 0.02$ & 9.99 & 52.40 & 0.38 & 5 \\
\hline 987 & 27 & $22.60 \pm 0.26$ & $0.34 \pm 0.16$ & 55.26 & 1.27 & 7 \\
\hline 991 & 24 & $22.33 \pm 0.40$ & $0.73 \pm 0.38$ & 54.00 & 0.76 & 4 \\
\hline 992 & 64 & $21.28 \pm 0.12$ & $0.57 \pm 0.73$ & 53.40 & 0.56 & 14 \\
\hline 993 & 42 & $21.85 \pm 0.12$ & $2.84 \pm 2.23$ & 53.40 & 0.70 & 9 \\
\hline 994 & 20 & $21.60 \pm 0.28$ & 9.99 & 52.40 & 0.62 & 5 \\
\hline 996 & 34 & $21.21 \pm 0.29$ & 9.99 & 53.40 & 0.50 & 7 \\
\hline 997 & 60 & $22.26 \pm 0.57$ & $1.14 \pm 0.30$ & 54.00 & 0.51 & 13 \\
\hline 998 & 31 & $22.15 \pm 0.68$ & 9.99 & 52.40 & 0.82 & 8 \\
\hline 999 & 96 & $22.66 \pm 0.49$ & $6.85 \pm 8.53$ & 54.00 & 0.78 & 23 \\
\hline 1000 & 321 & $21.93 \pm 0.69$ & $4.20 \pm 1.12$ & 54.25 & 1.18 & 28 \\
\hline 1001 & 67 & $22.08 \pm 0.75$ & $0.55 \pm 0.11$ & 54.30 & 0.80 & 15 \\
\hline 1002 & 158 & $22.18 \pm 0.86$ & $0.72 \pm 0.64$ & 53.70 & 0.78 & 18 \\
\hline 1003 & 68 & $21.72 \pm 0.17$ & $2.40 \pm 1.23$ & 53.40 & 0.70 & 17 \\
\hline
\end{tabular}

